\title{
LONG-TIME ASYMPTOTICS FOR SOLUTIONS OF THE NLS EQUATION WITH A DELTA POTENTIAL AND EVEN INITIAL DATA
}

\author{
PERCY DEIFT AND JUNGWOON PARK
}

\begin{abstract}
We consider the one-dimensional focusing nonlinear Schrödinger equation (NLS) with a delta potential and even initial data. The problem is equivalent to the solution of the initial/boundary problem for NLS on a half-line with Robin boundary conditions at the origin. We follow the method of Bikbaev and Tarasov which utilizes a Bäcklund transformation to extend the solution on the half-line to a solution of the NLS equation on the whole line. We study the asymptotic stability of the stationary 1-soliton solution of the equation under perturbation by applying the nonlinear steepest-descent method for Riemann-Hilbert problems introduced by Deift and Zhou. Our work strengthens, and extends, earlier work on the problem by Holmer and Zworski.
\end{abstract}

\section{INTRODUCTION}

The nonlinear Schrödinger (NLS) equation with an external potential $V(x)$ (Gross-Pitaevskii (GP) equation)

$$
i u_{t}+\frac{1}{2} \Delta u+V(x) u+\kappa|u|^{2} u=0, \quad \kappa= \pm 1
$$

arises as a model for a wide variety of phenomena in physics. In particular (see e.g. PS03]) equation (1.1) provides a model for the evolution of Bose-Einstein condensates in dilute boson gases at very low temperatures: in this case $V(x)$ is called the trapping potential and $\kappa=+1$ or -1 depending on whether the interaction of the bosons is attractive or repulsive, respectively. Furthermore, in this Bose-Einstein model, $|u(x)|^{2}$ is the boson density and $\mathcal{M}=\int|u(x)|^{2} d x$ is the total number of bosons present in the system. In the spatially inhomogeneous case $(V(x) \not \equiv c)$, exact solutions of (1.1) are hard to come by, even in one dimension. Further reduction in the one-dimensional case to the delta-potential at $x=0, V(x)=q \delta_{0}(x)$, still leaves a formidable and currently much studied problem (see e.g. [HZ09] and the references therein). However, as noted by Fokas, in one-dimension with $V(x)=q \delta_{0}(x), q \in \mathbb{R}$, and $u_{0}(x)=u(x, t=0)$ even, equation (1.1) 
becomes integrable. This is because the delta function introduces a jump in the derivative at $x=0, \frac{1}{2}\left(u_{x}(0+)-u_{x}(0-)\right)+q u(0)=0$, and if $u(x)$ is even this relation reduces to

$$
u_{x}(0+)+q u(0)=0
$$

In other words, (1.1) with even initial data reduces to the initialboundary value (IBV) problem for NLS on a half-line

$$
i u_{t}+\frac{1}{2} u_{x x}+|u|^{2} u=0, \quad x>0,
$$

with homogeneous boundary conditions (1.2) at $x=0$ : such problems are known to be integrable by an extension of the inverse scattering method (see [Skl87], Tar88], [Fok89], Kha91], [BT91] and [Tar91]).

In [HZ09 the authors consider the GP equation in one-dimension with $V=q \delta_{0}$ and $\kappa=1$,

$$
i u_{t}+\frac{1}{2} u_{x x}+q \delta_{0}(x) u+|u|^{2} u=0
$$

with initial data of the form

$$
u(x, 0)=v_{\lambda}(x)+w(x)
$$

where $q$ is small, $w(x)$ is even and of order $O(q)$, and $v_{\lambda}$ has the special form

$$
v_{\lambda}(x)=\lambda \operatorname{sech}\left(\lambda|x|+\tanh ^{-1}(q / \lambda)\right), \quad \lambda>|q| .
$$

The data $v_{\lambda}$ corresponds to the nonlinear ground state of the condensate obtained by minimizing the energy $\mathcal{H}=\int_{\mathbb{R}}\left[\frac{1}{2}\left|u_{x}\right|^{2}-q \delta_{0}|u|^{2}-\right.$ $\left.\frac{1}{2}|u|^{4}\right] d x$ subject to $\mathcal{M}=\int_{\mathbb{R}}|u(x)|^{2} d x=2(\lambda-q)$. Associated with $v_{\lambda}$, one has the stationary solution $u_{\lambda}(x, t)=e^{i \lambda^{2} t / 2} v_{\lambda}(x)$ for (1.4) corresponding to $w \equiv 0$. The main result in [HZ09] concerns the asymptotic stability of this ground state condensate under perturbations, $w=O(q), q \ll 1$. The authors in [HZ09] prove in particular that for $1 \leq t \leq c|q|^{-2 / 7}$

$$
u(0, t)=e^{i \hat{\lambda}^{2} t / 2}\left(\hat{\lambda}-\sqrt{\frac{2}{\pi t}} e^{i\left(\hat{\lambda}^{2} t / 2+\pi / 4\right)} \int_{0}^{\infty} w(x) d x\right)+O\left(q / t^{3 / 2}\right)
$$

for some explicit $\hat{\lambda}, \hat{\lambda} \sim v_{\lambda}(0)$ for $q$ small. The authors use PDEHamiltonian systems methods and do not utilize the integrability of the system explicitly. In this paper we analyze the above problem (1.4) (1.5), but now utilizing the full force of the integrability of the IBV problem together with the steepest-descent method for RiemannHilbert Problems (RHPs) in DZ93]DZ03. As indicated above, there are a number of different ways to show that the IBV problem (1.3) (1.2) 
is completely integrable. We will follow the method of Bikbaev and Tarasov [Tar88] BT91] Tar91], which is based in turn on Khabibullin Kha91. Part of this paper is devoted to reformulating the method of Bikbaev and Tarasov in the language of RHP's, so that the method in [DZ93] DZ03] can be applied.

Our main results are as follows. We use the standard notations of the scattering and inverse scattering method for the focusing NLS equation. We refer the reader, who may not be familiar with these notations, for example, $a(z)$, Bäcklund extension, etc., to Sections 3 and 4 below. We note that for $u(x, 0)=v_{\lambda}(x)+\epsilon w(x), w(x) \in H^{1,1}=\left\{w \in L^{2}(\mathbb{R}): w\right.$ is absolutely continuous, $\left.x w(x), w^{\prime} \in L^{2}(\mathbb{R})\right\}$, the equation (1.4) has a unique global (weak) solution in $H^{1,1}$, i.e. $t \mapsto u(t)=u(x, t)$ is a continuous map from $\mathbb{R}^{+}$into $H^{1,1}(\mathbb{R})$ satisfying

$$
\begin{aligned}
& u(t)=e^{-i H_{q} t / 2} u_{0}+i \int_{0}^{t} e^{-i H_{q}(t-s) / 2}|u(s)|^{2} u(s) \mathrm{d} s, \\
& u(t=0)=u_{0}=u_{0}(x) \in H^{1,1}(\mathbb{R}) .
\end{aligned}
$$

Here $H_{q}$ is the self-adjoint operator $-\frac{d^{2}}{d x^{2}}-2 q \delta_{0}$ on $\mathbb{R}$ with domain

$$
\begin{aligned}
D\left(H_{q}\right)=\left\{f \in L^{2}:\right. & f \text { is absolutely continuous, } \\
& f^{\prime} \text { is absolutely continuous in } \mathbb{R} \backslash\{0\}, \\
& \left.f^{\prime}, f^{\prime \prime} \in L^{2}, f^{\prime}(0+)-f^{\prime}(0-)+2 q f(0)=0\right\} .
\end{aligned}
$$

We will also consider global weak solutions $u(t)$ of $(1.3)$ with boundary conditions (1.2) in the sense that $u(t)$ is a continuous map from $\mathbb{R}^{+}$ into $H^{1,1}\left(\mathbb{R}^{+}\right)=\left\{f \in L^{2}\left(\mathbb{R}^{+}\right): f\right.$ is absolutely continuous $, f^{\prime}, x f \in$ $\left.L^{2}\left(\mathbb{R}^{+}\right)\right\}$which solves

$$
\begin{aligned}
& u(t)=e^{-i H_{q}^{+} t / 2} u_{0}+i \int_{0}^{t} e^{-i H_{q}^{+}(t-s) / 2}|u(s)|^{2} u(s) \mathrm{d} s, \\
& u(t=0)=u_{0}=u_{0}(x) \in H^{1,1}\left(\mathbb{R}^{+}\right) .
\end{aligned}
$$

Here $H_{q}^{+}$is the self-adjoint operator $-\frac{d^{2}}{d x^{2}}$ on $\mathbb{R}^{+}$with domain

$$
\begin{array}{r}
D\left(H_{q}^{+}\right)=\left\{f \in L^{2}\left(\mathbb{R}^{+}\right): f \text { and } f^{\prime}\right. \text { are absolutely continuous, } \\
\left.f^{\prime \prime} \in L^{2}\left(\mathbb{R}^{+}\right), f^{\prime}(0+)+q f(0)=0\right\} .
\end{array}
$$

Definition 1.1. We say that $u(t)$ solves a $\mathrm{HNLS}_{q}^{+}$if $u(t)$ is a (global, weak) solution to (1.3) with (1.2). If $u(t)$ solves $(1.3)$ on $\mathbb{R}^{-}$with (1.2), we say that $u(t)$ solves a $\mathrm{HNLS}_{q}^{-}$.

Also we will consider global weak solutions $u(t)$ of NLS (3.1) on the line, by which we mean a continuous map from $\mathbb{R}^{+}$into $H^{1,1}(\mathbb{R})$ such 
that

$$
\begin{aligned}
& u(t)=e^{-i H_{0} t / 2} u_{0}+i \int_{0}^{t} e^{-i H_{0}(t-s) / 2}|u(s)|^{2} u(s) \mathrm{d} s, \\
& u(t=0)=u_{0} \in H^{1,1}(\mathbb{R}) .
\end{aligned}
$$

Here $H_{0}$ is the self-adjoint operator $-\frac{d^{2}}{d x^{2}}$ on $\mathbb{R}$ with domain

$$
D\left(H_{0}\right)=\left\{f \in L^{2}: f \text { and } f^{\prime} \text { are absolutely continuous, } f^{\prime}, f^{\prime \prime} \in L^{2}\right\} .
$$

Unless stated otherwise, whenever we discuss a solution of (1.4) in $H^{1,1}(\mathbb{R})$, (1.3) with boundary conditions $(1.2)$ in $H^{1,1}\left(\mathbb{R}^{+}\right)$, or (3.1) in $H^{1,1}(\mathbb{R})$, we always mean the global weak solutions described above. On a number of occasions, however, particularly in our motivations for the Bikbaev and Tarasov's method, we will also consider classical solutions to $\mathrm{HNLS}_{q}^{+}$, etc., i.e., the solutions which are $C^{2}$ with respect to $x$ and $C^{1}$ with respect to $t$. In addition, on a few occasions, we will also need to consider solutions of NLS in $H^{k, k}(\mathbb{R}), k \geq 1$, where $H^{k, j}=\{u \in$ $L^{2}(\mathbb{R}): u, u^{\prime}, \cdots, u^{(k-1)}$ are absolutely continuous, $u^{(k)}(x), x^{j} u(x) \in$ $\left.L^{2}(\mathbb{R})\right\}, k \geq 0, j \geq 0$. We will show that (1.8), (1.9) and (1.10) indeed have unique solutions in $H^{1,1}$, in Section 2 below. Moreover, the solutions $u(t)=u\left(t ; u_{0}\right)$ of these equations depend on the initial data $u_{0}$, uniformly for $t$ in compact subsets of $\{t \geq 0\}$.

Theorem 1 (Asymptotics of $u(x, t)$ as $t \rightarrow \infty)$. Let $0<|q|<\mu_{0}$. Suppose that $u(x, t)$ is the (unique, weak, global) solution of the equation (1.4) with initial data

$$
u(x, 0)=v_{\mu_{0}}(x)+\epsilon w(x),
$$

where $w$ is even and $\|w\|_{H^{1,1}(\mathbb{R})} \leq c$. Let $u_{0}^{e}(x)$ be the Bäcklund extension of $\left.u(x, 0)\right|_{\mathbb{R}^{+}}$to $\mathbb{R}$ with respect to $q$. Let $a(z)$ and $r(z)$ be the scattering function and the reflection coefficient of $u_{0}^{e}(x)$, respectively. Denote $z_{0}=|x| / t$. Fix $0<\kappa<\frac{1}{4}$ and $M>1$. Then, there exists an $\chi_{0}=\chi_{0}\left(\mu_{0}\right)>0$ such that the following holds: For any $0 \leq \epsilon \leq \chi_{0}|q|^{\frac{1}{2}}$, $a(z)$ has one simple zero if $q>0$ and at most two simple zeros for $q<0$. In both cases the zeros lie in $i \mathbb{R}^{+}$. We denote the zeros by $z_{1}=i \mu_{1}, z_{2}=i \mu_{2}$. Set

$$
l_{j}=\frac{1}{\pi} \int_{0}^{\infty} \frac{s}{s^{2}+\mu_{j}^{2}} \log \left(1+|r(s)|^{2}\right) \mathrm{d} s,
$$

and

$$
\hat{\delta}_{j}=\exp \left[\frac{1}{2 \pi i} \int_{0}^{z_{0}} \frac{\log \left(1+|r(s)|^{2}\right)}{s-i \mu_{j}} \mathrm{~d} s\right] .
$$




$$
\begin{aligned}
& v_{j}=-\sqrt{\frac{\mu_{j}-q}{\mu_{j}+q}} e^{i\left(\mu_{j}^{2} t / 2+\rho_{j}+l_{j}\right)} \hat{\delta}_{j}^{2}, \quad \hat{v}_{j}=\frac{\mu_{j}+q}{\mu_{j}-q} v_{j}, \\
& v_{j}^{x}=v_{j} e^{-\mu_{j}|x|}, \quad \hat{v}_{j}^{x}=\hat{v}_{j} e^{-\mu_{j}|x|},
\end{aligned}
$$

for $j=1,2$, where $\rho_{1}$ is described in (17.28) if a $(z)$ has one zero, and $\rho_{1}, \rho_{2}$ are given in (7.32) if a $(z)$ has two zeros. Let $k_{1}, k_{2}, p_{1}, p_{2}, p_{3}$ and $p_{4}$ be given in (6.12), (6.14), (7.30) and (7.35).

(i) Suppose that $a(z)$ has one simple zero at $z_{1}=i \mu_{1} \in i \mathbb{R}^{+}$as given in (7.26),

- For $|x| \leq M$ and $t \geq 1$ :

$$
u(x, t)=e^{i\left(\mu_{1}^{2} t / 2+\rho_{1}+l_{1}\right)} v_{\mu_{1}}(x)+O\left(\epsilon|q|^{-\frac{1}{2}} t^{-\left(\frac{1}{2}+\kappa\right)}\right) .
$$

- For $|x| \geq 1 / M$ and $t \geq 1$ :

$$
u(x, t)=-\frac{k_{1}}{\sqrt{t}}-\frac{2 \mu_{1}\left(v_{1}^{x}+p_{1}\right)\left(\overline{p_{2}} \overline{v_{1}^{x}}+1\right)}{\left|v_{1}^{x}+p_{1}\right|^{2}+\left|p_{2} v_{1}^{x}+1\right|^{2}}+O\left(\epsilon|q|^{-\frac{1}{2}} t^{-\left(\frac{1}{2}+\kappa\right)}\right),
$$

(ii) If a $(z)$ has two simple zeros at $z_{1}=i \mu_{1}, z_{2}=i \mu_{2}\left(\mu_{1}>\mu_{2}\right)$, set $\tau \equiv|q| \sqrt{t}$.

- For $|x| \leq M$, as $\tau \rightarrow \infty$ :

$$
\begin{aligned}
u(x, t)= & e^{i\left(\mu_{1}^{2} t / 2+\rho_{1}+l_{1}\right)} \mu_{1} \operatorname{sech}\left(\mu_{1} x-\tanh ^{-1}\left(q / \mu_{1}\right)\right) \\
& -\frac{2 \mu_{2}\left(\hat{v}_{2}^{x}-s_{0}\right)\left(1+\hat{v}_{1}^{x} \overline{s_{0}}\right)}{\left|\hat{v}_{2}^{x}-s_{0}\right|^{2}+\left|1+\hat{v}_{1}^{x} \overline{s_{0}}\right|^{2}}+O\left(\epsilon|q|^{-\frac{1}{2}} t^{-\left(\frac{1}{2}+\kappa\right)}\right),
\end{aligned}
$$

where

$$
s_{0}=-\frac{2 \mu_{1}}{\mu_{1}-\mu_{2}} \frac{\hat{v}_{1}^{x}-\hat{v}_{2}^{x}}{\left|\hat{v}_{1}^{x}\right|^{2}+1} .
$$

- For $|x| \geq 1 / M$, as $\tau \rightarrow \infty$ :

$$
\begin{aligned}
u(x, t)= & -\frac{k_{1}}{\sqrt{t}}-\frac{2 \mu_{1}\left(\hat{v}_{1}^{x}+p_{1}\right)\left(\overline{p_{2}} \overline{\hat{v}_{1}^{x}}+1\right)}{\left|\hat{v}_{1}^{x}+p_{1}\right|^{2}+\left|p_{2} \hat{v}_{1}^{x}+1\right|^{2}}-\frac{2 \mu_{2} s_{1} \overline{s_{2}}}{\left|s_{1}\right|^{2}+\left|s_{2}\right|^{2}} \\
& +O\left(\epsilon q e^{-\tau^{2}}+\epsilon^{2} q\left(z_{0}^{2}+q^{2}\right)^{-1} t^{-1}+\epsilon|q|^{-\frac{1}{2}} t^{-\left(\frac{1}{2}+\kappa\right)}\right) .
\end{aligned}
$$

where

$$
s=\frac{2 \mu_{1}}{\mu_{1}-\mu_{2}} \frac{\left(p_{2} \hat{v}_{1}^{x}+1\right)\left(\hat{v}_{2}^{x}+p_{3}\right)-\left(\hat{v}_{1}^{x}+p_{1}\right)\left(p_{4} \hat{v}_{2}^{x}+1\right)}{\left|\hat{v}_{1}^{x}+p_{1}\right|^{2}+\left|p_{2} \hat{v}_{1}^{x}+1\right|^{2}}
$$

and

$$
\begin{aligned}
& s_{1}=\hat{v}_{2}^{x}+p_{3}-\left(\overline{p_{2}} \overline{\hat{v}_{1}^{x}}+1\right) s, \\
& s_{2}=p_{4} \hat{v}_{2}^{x}+1+\left(\overline{\hat{v}_{1}^{x}}+\overline{p_{1}}\right) s .
\end{aligned}
$$


Remark 1.2. This result should be compared with [FIS05] where the authors obtain the long-time asymptotics for the solution of NLS on half-line using the method introduced by Fokas [Fok02].

Remark 1.3. For $q<0$, case (ii) is generic in the sense that there exists an open dense subset $\mathcal{U} \subset H^{1,1}\left(\mathbb{R}^{+}\right)$such that any $w \in \mathcal{U}$ falls into case (ii) for all sufficiently small $\epsilon>0$.

Remark 1.4. If $\epsilon=0$, we have $\mu_{1}=\mu_{0}, \rho_{1}=0, r(z) \equiv 0$ and hence $l_{1}=0, \hat{\delta}_{1}=1$. This matches the exact solution $u(x, t)=e^{i \mu_{0}^{2} t / 2} v_{\mu_{0}}(x)$ for $x \in \mathbb{R}$.

Remark 1.5. If the initial data has sufficient smoothness and decay, the error term can be sharpened to order $O\left(\epsilon|q|^{-\frac{1}{2}} \frac{\log t}{t}\right)$ in (1.14), (1.15) and (1.16).

Remark 1.6. As we will see in the proof of the Theorem, (1.15) and (1.18) hold for all $x \in \mathbb{R}$. In the overlapping region $1 / M \leq|x| \leq M$, as $z_{0}=O\left(t^{-1}\right)$,

$$
\hat{\delta_{1}}=1+O\left(\epsilon^{2} t^{-1}\right) \text { and } k_{1}=O\left(r\left(z_{0}\right)\right)=O\left(\epsilon|q|^{-\frac{1}{2}} t^{-\frac{1}{2}}\right),
$$

and hence $p_{1}, p_{2}=O\left(\epsilon|q|^{-\frac{1}{2}} t^{-1}\right)$. This implies that (1.14) and (1.15) are consistent. In the case that $a(z)$ has two zeros, it is easily verified that (1.16) and (1.18) match as well.

Remark 1.7. It can be verified that the leading terms in (1.16) represent the 2-soliton solution for NLS (cf. [FT87]).

When $\epsilon=q$, the following result recovers, in particular, (1.7) and provides a more detailed description of the asymptotics of $u(x, t)$ for all $t \ll q^{-2}$.

Theorem 2 (Asymptotics of $u(x, t)$ for $t \ll q^{-2}, q$ small). Suppose $u(x, t)$ solves (1.4) with initial data

$$
u(x, 0)=v_{\mu_{0}}(x)+q w(x),
$$

where $w(x)$ is real, even and $\|w\|_{H^{1,1}(\mathbb{R})} \leq c$. Let $u_{0}^{e}(x)$ be the Bäcklund extension of $\left.u(x, 0)\right|_{\mathbb{R}^{+}}$to $\mathbb{R}$ with respect to $q$ and let $a(z)$ be the scattering function of $u_{0}^{e}(x)$. Define

$$
\begin{aligned}
& w_{0}=\int_{0}^{\infty} w(s) \mathrm{d} s, \quad w_{1}=\int_{-\infty}^{\infty} w(s) v_{\mu_{0}}(s) \mathrm{d} s \\
& K(z)=-\operatorname{Re} \int_{0}^{\infty} e^{-i s z} w(s) \frac{z^{2}-2 i z \mu_{0} \tanh \left(\mu_{0} s\right)-\mu_{0}^{2}}{z^{2}+\mu_{0}^{2}} \mathrm{~d} s .
\end{aligned}
$$

Denote $\tau=|q| \sqrt{t}$ and $z_{0}=|x| / t$. Fix $C, M>1$. Then, there exists a $q_{0}=q_{0}\left(\mu_{0}\right)$ such that the following holds: For any $0<|q|<q_{0}, a(z)$ 
has one simple zero if $q>0$ and at most two simple zeros for $q<0$. In both cases the zeros lie in $i \mathbb{R}^{+}$. We denote the zeros by $z_{1}=i \mu_{1}$, $z_{2}=i \mu_{2}$. Set

$$
v_{1}=-\sqrt{\frac{\mu_{1}-q}{\mu_{1}+q}} e^{i \mu_{1}^{2} t / 2}, \quad v_{1}^{x}=v_{1} e^{-\mu_{1}|x|},
$$

Let $p_{1}$ and $p_{2}$ be given in (8.5).

(i) If $a(z)$ has one simple zero at $z_{1}=i \mu_{1} \in i \mathbb{R}^{+}$, then $\mu_{1}=\mu_{0}+q w_{1}+$ $O\left(q^{2}\right)$. Moreover,

- for the region $|x| \leq M$ :

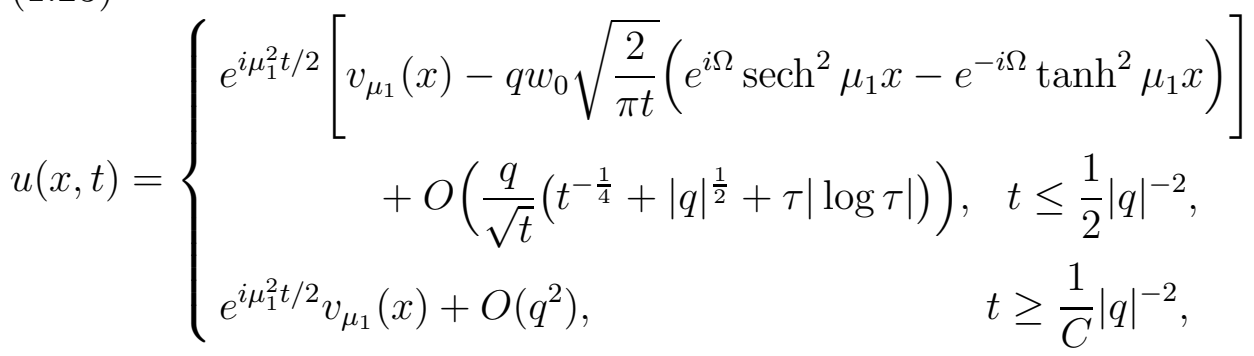

where $\Omega(x, t)=-x^{2} /(2 t)+\mu_{1}^{2} t / 2+\pi / 4$.

- For $|x| \geq 1 / M$ :

$$
u(x, t)=\left\{\begin{array}{cc}
i\left(z_{0}-i \mu_{1}\right) p_{1}-\frac{2 \mu_{1}\left(v_{1}^{x}+p_{1}\right)\left(\overline{p_{2}} \overline{v_{1}^{x}}+1\right)}{\left|v_{1}^{x}+p_{1}\right|^{2}+\left|p_{2} v_{1}^{x}+1\right|^{2}} & \\
+O\left(\frac{q}{\sqrt{t}}\left(t^{-\frac{1}{4}}+|q|^{\frac{1}{2}}+\tau|\log \tau|\right)\right), & t \leq \frac{1}{2}|q|^{-2}, \\
e^{i \mu_{1}^{2} t / 2} v_{\mu_{1}}(x)+O\left(q^{2}\right), & t \geq \frac{1}{C}|q|^{-2} .
\end{array}\right.
$$

(ii) If a $(z)$ has two simple zeros $z_{1}=i \mu_{1} \in i \mathbb{R}^{+}$and $z_{2}=i \mu_{2} \in i \mathbb{R}^{+}$: necessarily $q<0$. In this case, $\mu_{1}=\mu_{0}+q w_{1}+O\left(q^{2}\right)$ and $\mu_{2}=$ $-q+O\left(q^{3}\right)$. Moreover, the asymptotic behavior of $u(x, t)$ is the same as in one-zero case up to error estimates in (1.23) and (1.24).

Remark 1.8. As we will see in the proof of the Theorem, (1.24) holds for all $x \in \mathbb{R}$.

Remark 1.9. The results in Theorem 1 and Theorem 2 are complementary to each other. The asymptotic form (1.14), for example, provides a decay rate in the error term as $t \rightarrow \infty$ whereas (1.23) does not. If $t \ll|q|^{-2}$, however,

$$
\epsilon|q|^{-\frac{1}{2}} t^{-\left(\frac{1}{2}+\kappa\right)} \geq|q|^{\frac{1}{2}} t^{-\frac{3}{4}} \gg \frac{|q|}{\sqrt{t}}
$$


Hence the solution is described in greater detail by (1.23) in this region.

Remark 1.10. The above result shows that, in both cases, whether $a(z)$ has one or two zeros, the leading terms are the same for $t \ll|q|^{-2}$. In particular, in the case that $a(z)$ has two zeros, the contribution from the second zero is not noticeable for $t \ll|q|^{-2}$. The one-zero case and the two-zero case indeed behave differently, however, for larger times, $t \gg|q|^{-2}$ (see Theorem 11). Hence the critical region where the solution changes its behavior is $t \sim q^{-2}$.

Remark 1.11. The determining role played by the parameter $\tau=|q| \sqrt{t}$ can be traced, at least at the formal level, to the following observation: the Bäcklund transformation taking NLS from the half line to the full line introduces terms of the form $\frac{q}{z \pm i q}$, which are small away from $z=\mp q$. On the other hand the solution of the Cauchy problem involves integrals of the form $\int f(z) \frac{q}{z \pm i q} e^{-i t z^{2} / 2}$, from which we see that the leading order contributions take the form $\sim f(\mp i q) e^{i t q^{2} / 2}=f(\mp i q) e^{i \tau^{2} / 2}$, in which the role of $\tau$ is prominently displayed.

Remark 1.12. Note that in the cross-over region, $\frac{1}{C} \leq \tau \leq C$ the formulae (1.16), (1.18), (1.23) and (1.24) describe the solution only up to an error $O\left(q^{2}\right)=O\left(t^{-1}\right)$.

Remark 1.13. Holmer and Zworski [HZ09] used $\hat{\lambda}=\mu_{0}+q w_{1}$ in (1.7) to describe the evolution of solutions to (1.4) for time interval $1 \ll t \leq$ $c|q|^{-2 / 7}\left(\ll c|q|^{-2}\right)$. From (1.23) we see that as $\mu_{1}=\hat{\lambda}+O\left(q^{2}\right)$,

$$
\begin{aligned}
u(0, t) & =e^{i \mu_{1}^{2} t / 2}\left(v_{\mu_{1}}(0)-q w_{0} \sqrt{\frac{2}{\pi t}} e^{i\left(\mu_{1}^{2} t / 2+\pi / 4\right)}\right)+o\left(\frac{q}{\sqrt{t}}\right) \\
& =e^{i \hat{\lambda}^{2} t / 2}\left(v_{\hat{\lambda}}(0)-q w_{0} \sqrt{\frac{2}{\pi t}} e^{i\left(\hat{\lambda}^{2} t / 2+\pi / 4\right)}\right)+o\left(\frac{q}{\sqrt{t}}+q^{2} t\right),
\end{aligned}
$$

which agrees to leading order with the result (1.7) of [HZ09] for times $t \ll|q|^{-2 / 3}$. Of course $|q|^{-2 / 7} \ll|q|^{-2 / 3}$, and we see in particular that the result in [HZ09] is in fact true in the larger interval $1 \ll t \ll|q|^{-2 / 3}$. In order to describe the asymptotics up to time $t \sim|q|^{-2}$, however, we need to use the more accurate frequency $\mu_{1}^{2}$ in place of $\hat{\lambda}^{2}$ in (1.7).

In order to gain some perspective on the nonlinear problem (1.3) (1.2), it is useful to consider first the linear case:

$$
\begin{aligned}
& i u_{t}+\frac{1}{2} u_{x x}=0, \quad x \geq 0, \quad t \geq 0, \\
& u(x, 0)=u_{0}(x), \quad x>0, \\
& u_{x}(0+, t)+q u(0, t)=0, \quad t \geq 0 .
\end{aligned}
$$


for some $q \in \mathbb{R}$. Here we assume that $u_{0}(x)$ is smooth and decays at some appropriate rate as $x \rightarrow \infty$. The standard, functional analytic way to solve this problem utilizes the self-adjoint operator $H_{q}^{+}=$ $-d^{2} / d x^{2}$ acting in $L^{2}(0, \infty)$ with the domain $D\left(H_{q}^{+}\right)=\left\{u \in L^{2}(0, \infty)\right.$ : $u, u^{\prime}$ absolutely continuous, $\left.u^{\prime \prime} \in L^{2}(0, \infty), u_{x}(0+)+q u(0+)=0\right\}$. For $q<0, H_{q}^{+}$has purely absolutely continuous spectrum on $(0, \infty)$, but for $q>0, H_{q}^{+}$has, in addition, an $L^{2}\left(\mathbb{R}^{+}\right)$eigenvalue at $-q^{2}$. The solution $u(x, t)$ of $(1.25)$ is then expressed in terms of the spectral theory of $H_{q}^{+}$as follows: for $q<0$,

$$
u(x, t)=\int_{0}^{\infty} e^{-i t z^{2} / 2} r(z) f_{q}(x, z) \mathrm{d} z,
$$

and for $q>0$,

$$
\begin{aligned}
u(x, t) & =\int_{0}^{\infty} e^{-i t z^{2} / 2} r(z) f_{q}(x, z) \mathrm{d} z \\
& +2 q e^{i t q^{2} / 2} e^{-q x} \int_{0}^{\infty} e^{-q y} u_{0}(y) \mathrm{d} y .
\end{aligned}
$$

Here $r(z)$ is determined by the initial data $u_{0}(x)$ and the functions $f_{q}(x, z)=\frac{e^{i x z}}{i z+q}-\frac{e^{-i x z}}{-i z+q}$ are the continuum eigenfunctions of $H_{q}^{+}$.

In the nonlinear case on the whole line, $-\infty<x<\infty$, the method of Zakharov-Shabat [ZS72] shows how to solve NLS in terms of the spectral theory of the associated Lax operator $\partial_{x}-L$ where

$$
L=i z \sigma+\left(\begin{array}{cc}
0 & u(x) \\
-u(x) & 0
\end{array}\right), \quad \sigma=\frac{1}{2} \sigma_{3}, \quad \sigma_{3}=\left(\begin{array}{cc}
1 & 0 \\
0 & -1
\end{array}\right) .
$$

On the half line, $x>0$, the method of Fokas [Fok89] provides, in an appropriate sense, an " $H_{q}^{+}$-analog" of $L$, and hence a solution procedure for $\mathrm{HNLS}_{q}^{+}$. Alternatively, however, we can try to solve the IBV problem by an analog of the "method of images" from electrostatics, i.e. we extend the solution $u(x, t)$ to a solution $u_{e}(x, t)$ of the differential equation on the whole real line, in such a way that the boundary condition at $x=0$ is automatically satisfied.

In particular for the linear problem (1.25) we seek a $C^{1}$ function $u_{e}(x, t)$ that decays appropriately as $|x| \rightarrow \infty$ on $\mathbb{R}$ such that

(i) $u_{e}(x, t)$ solves the Schrödinger equation $i \partial_{t} u_{e}+\frac{1}{2} \partial_{x}^{2} u_{e}=0$ on $\mathbb{R} \times \mathbb{R}^{+}$

(ii) $u_{e}(x, t=0)=u_{0}(x), x>0$,

(iii) $\left(u_{e}\right)_{x}(x, t)+q u_{e}(x, t)$ is an odd function, $t \geq 0$.

Condition (iii) immediately implies that $\left(u_{e}\right)_{x}(0, t)+q u_{e}(0, t)=0$ and so for $x>0, u_{e}(x, t)$ is a solution to (1.25) with $u_{e}(x, 0)=u_{0}(x), x>0$. 
To ensure (iii) for all $t \geq 0$, it is sufficient, by linearity and homogeneity, to verify condition (iii) at $t=0$, i.e.

$$
\left(u_{e}\right)_{x}(-x, 0)+q u_{e}(-x, 0)=-\left(\left(u_{e}\right)_{x}(x, 0)+q u_{e}(x, 0)\right), \quad x>0 .
$$

Integrating this relation we obtain

$$
u_{e}(-x, 0)=u_{0}(x)+2 q \int_{0}^{x} e^{q(x-s)} u_{0}(s) \mathrm{d} s, \quad x>0 .
$$

Set

$$
u_{e}(x, 0)= \begin{cases}u_{0}(x), & x>0, \\ u_{0}(-x)+2 q \int_{0}^{-x} e^{-q(x+s)} u_{0}(s) \mathrm{d} s, & x<0 .\end{cases}
$$

A direct calculation shows that $u_{e}(x, 0)$ is indeed $C^{1}$ and gives rise, in principle, to a solution of the Schrödinger equation satisfying (i)(ii)(iii) and hence a solution of (1.25).

There are, however, some technical considerations. If $q<0$, then one sees from (1.29) that $u_{e}(x, 0)$ decays (at a rate dictated by $q$ and $u_{0}(x)$ ) as $x \rightarrow-\infty$. This means, in particular, that $u_{e}(x, t)$ solves a PDE on $\mathbb{R}$ with initial data in some standard class for which existence and uniqueness are easily established. Indeed, one can solve (i)(ii)(iii) using Fourier theory (equivalently, using the spectral theory of $H=-d^{2} / d x^{2}$ in $L^{2}(\mathbb{R})$ )

$$
u_{e}(x, t)=\frac{1}{2 \pi} \int_{\mathbb{R}} e^{i\left(x z-t z^{2} / 2\right)} \hat{u}(z) \mathrm{d} z
$$

where $\hat{u}(z)$ decays appropriately as $|z| \rightarrow \infty$. To ensure the oddness of $\left(u_{e}\right)_{x}(x, t)+q u_{e}(x, t)$, we see that $r(z) \equiv(i z+q) \hat{u}(z)$ must be odd, from which it follows that

$$
r(z)=\int_{\mathbb{R}} e^{-i x z} F(x) \mathrm{d} x
$$

where $F(x)=\left(u_{0}\right)_{x}(x)+q u_{0}(x), x>0$ and $F(x)=-F(-x)$ for $x<0$. To check (ii), we note that for $x>0,\left(u_{e}\right)_{x}(x, 0)+q u_{e}(x, 0)=$ $\int e^{i x z} r(z) \mathrm{d} z=\left(u_{0}\right)_{x}(x)+q u_{0}(x)$ and so $e^{q x} u_{e}(x, 0)=e^{q x} u_{0}(x)+c$. Letting $x \rightarrow+\infty$, we see that $c=0$ and so $u_{e}(x, 0)=u(x)$ for $x>0$. Thus,

$$
\begin{aligned}
u_{e}(x, t) & =\int_{\mathbb{R}} e^{i\left(x z-t z^{2} / 2\right)} \frac{r(z)}{i z+q} \mathrm{~d} z \\
& =\int_{0}^{\infty} e^{-i t z^{2} / 2} r(z)\left(\frac{e^{i x z}}{i z+q}-\frac{e^{-i x z}}{-i z+q}\right) \mathrm{d} z
\end{aligned}
$$


where $r(z)$ is determined from (1.32). In other words the method of images leads us back to the functional analytical approach using the operator $H_{q}^{+}$. If $q>0$, however, $u_{e}(x, 0) \sim e^{q|x|} \int_{0}^{\infty} e^{-q s} u_{0}(s) \mathrm{d} s$ as $x \rightarrow-\infty$ so that generically $u_{e}(x, 0)$ does not decay as $x \rightarrow-\infty$. Thus the method of images breaks down. Said differently, the functional analytic solution procedure (1.27) for $q>0$ cannot be "unfolded" to a "method of images" on the whole line with $u_{e}(x)$ sufficiently smooth and decaying.

The method of [Tar88] [BT91] Tar91] to solve $\mathrm{HNLS}_{q}^{+}$is a nonlinear analog of the "method of images": we seek an extension $u_{e}(x, t)$ of the solution $u(x, t)$ of (1.3) such that $u_{e}(x, t)$ solves NLS, $i \partial_{t} u_{e}+\frac{1}{2} \partial_{x}^{2} u_{e}+$ $\left|u_{e}\right|^{2} u_{e}=0$, on the whole line and the boundary condition $\left(u_{e}\right)_{x}(0, t)+$ $q u_{e}(0, t)=0$ is automatically satisfied. As NLS is not linear (and also not homogeneous), choosing $\left(u_{e}\right)_{x}(x, 0)+q u_{e}(x, 0)$ to be odd no longer works. The situation is more subtle: The key ingredient in [Tar88] [BT91] Tar91] is the notion of a Bäcklund transformation. As we will show (see Remark4.31 below), the method of [Tar88] BT91] [Tar91] is not just a nonlinear analog of the method of images for (1.25), but in fact a nonlinearization of the method in an appropriate sense.

We recall (see e.g. [RS02]) that a Bäcklund transformation refers very generally to a mapping $\mathcal{B}$ whereby a solution, say $u$, of one differential equation is transformed into a solution, say $v=\mathcal{B}(u)$, of another equation. The two equations may be the same in which case $\mathcal{B}$ is called an auto-Bäcklund transformation. Bäcklund transformations have their origin in the transformation theory of pseudospherical surfaces by Bianchi and Bäcklund in the 1880's. (For such surfaces the equation that underlies the theory is the sine-Gordon equation (see [RS02]).) In the modern theory of integrable system, in particular, the Bäcklund transformation has emerged as a major tool. The map $u \mapsto \mathcal{B}(u) \equiv-u$ is a trivial auto-Bäcklund transformation for NLS: More interesting, for example, is the transformation (see e.g. [Dei78]) $w \mapsto W$ where

$$
\begin{aligned}
& W(x, t)=-2 \frac{d^{2}}{d x^{2}} \log w(x, t), \\
& w(x, t)=e^{\theta}+\alpha e^{-\theta}, \quad \alpha>0, \quad \theta=\beta x-4 \beta^{3} t, \quad \beta \in \mathbb{R},
\end{aligned}
$$

which takes the solution $w(x, t)$ of the (integrable!) linear equation

$$
w_{t}+4 w_{x x x}=0
$$

into a (soliton) solution $W(x, t)$ of the $\mathrm{KdV}$ equation

$$
W_{t}-6 W W_{x}+W_{x x x}=0
$$


The idea in Tar88 BT91]Tar91 is to use a Bäcklund transformation to extend the solution $u(x, t)$ in $x>0$ to NLS (1.3) to a solution $u_{e}(x, t)$ on the whole line, in such a way that the boundary condition $u_{e}(0, t)+q u_{e}(0, t)=0$ is automatically satisfied, as desired. In addition the transformation should be such, as in the linear case, that the extension $u_{e}(x, t)$ is smooth and decays as $x \rightarrow \pm \infty$ so that the standard methods of scattering/inverse scattering theory, and hence RiemannHilbert methods can be applied. Riemann-Hilbert theory provides the precise analog in the nonlinear case of the Fourier transform method given in (1.31). As in the linear case (cf. $q>0$ ), however, there are limitations on the applicability of this "method of images." (see Remark 4.37 below)

The paper is organized as follows. In Section 2, we prove the existence of solutions of equations (1.8), (1.9) and (1.10). In Section 3, we describe the scattering and inverse scattering method for the focusing NLS equation on the line using Riemann-Hilbert techniques. In Section 4, we discuss Bäcklund transformation and the method of Bikbaev and Tarasov to solve NLS on the half-line with boundary conditions (1.2). A significant part of this section involves the rephrasing of the method of Bikbaev and Tarasov as a RHP. In Sections 5, 6 and 7, we apply the steepest-descent method for RHP in DZ93 DZ03 to analyze $u(x, t)$ as $t \rightarrow \infty$. (cf. Theorem 1) Finally in Section 8 we combine the results from Section 4 together with Riemann-Hilbert method to obtain a more detailed description of the solution $u(x, t)$ for times $t=O\left(q^{-2}\right)$ (cf. Theorem 2).

We need to clarify a point of terminology. We have defined a Bäcklund transformation very generally as a mapping which takes solutions of one differential equation into the solution of another differential equations. In particular, by (4.10), the map $\psi \mapsto \tilde{\psi}=(z+P) \psi$ is a Bäcklund transformation taking solutions of $\left(\partial_{x}-L\right) \psi=0$ to solutions of $\left(\partial_{x}-\tilde{L}\right) \tilde{\psi}=$ 0 . But (4.10) also shows that $\partial_{x}-\tilde{L}=(z+P)\left(\partial_{x}-L\right)(z+P)^{-1}$ is invertible if and only if $\partial_{x}-L$ is invertible (apart, possibly, from points $z$ in the $\operatorname{spec}(-\mathrm{P}))$. Said differently, the operators $(i \sigma)^{-1}\left(\partial_{x}-Q\right)$ and $(i \sigma)^{-1}\left(\partial_{x}-\tilde{Q}\right)$ are iso-spectral (modulo points in spec $(-\mathrm{P})$ ). Such iso-spectral transformations are usually referred to in the inverse scattering community as Darboux transformations. There is considerable freedom in the choice of Darboux transformations, and we see that the Bäcklund transformation that we utilize in this paper taking solutions of NLS to solutions of NLS, are constructed from Darboux transformations $\partial_{x}-L \mapsto \partial_{x}-\tilde{L}$ with a specific choice of parameters. The choice of parameters in the Darboux transformations, depends in general, on 
the tack at hand. For example, a different choice of parameters gives rise to an operator $\partial_{x}-\tilde{L}$ with poles added in or removed from the spectrum of $\partial_{x}-L$. Such Darboux transformations are discussed in the appendix.

\section{Solutions of EQuations (1.8), (1.9) AND (1.10)}

Theorem 3. Solutions to equations (1.8), (1.9) and (1.10) in $H^{1,1}(\mathbb{R})$, $H^{1,1}\left(\mathbb{R}^{+}\right)$and $H^{1,1}(\mathbb{R})$, respectively, exist and are unique for all $t \geq 0$.

The proof of this theorem is (essentially) standard. For the convenience of the reader we provide some of the details for $\operatorname{HNLS}_{q}^{+}$(1.9). We proceed in steps. Let $U_{t} \equiv e^{-i H_{q}^{+} t / 2}$ be the one parameter unitary operator generated by $H_{q}^{+}$in $L^{2}\left(\mathbb{R}^{+}\right)$. If $u(t)=u(x, t)$ solves (1.9), set

$$
\breve{u}(t) \equiv U_{-t} u(t)=u_{0}+i \int_{0}^{t} U_{-s}|u(s)|^{2} u(s) \mathrm{d} s .
$$

For any $\phi \in C_{0}^{\infty}(0, \infty)$, as $U_{t}$ is unitary in $L^{2}\left(\mathbb{R}^{+}\right)$,

$$
\begin{aligned}
\frac{d}{d t}(\phi, u(t)) & =\frac{d}{d t}\left(U_{-t} \phi, \breve{u}(t)\right) \\
& =\left(-\frac{i}{2} U_{-t} H_{q}^{+} \phi, \breve{u}(t)\right)+\left(U_{-t} \phi, i U_{-t}|u(t)|^{2} u(t)\right),
\end{aligned}
$$

where $(f, g)=\int_{0}^{\infty} \bar{f} g$ is the standard inner product in $L^{2}\left(\mathbb{R}^{+}\right)$. Thus,

$$
\frac{d}{d t}(\phi, u(t))=\frac{i}{2}\left(\phi^{\prime \prime}, u\right)+i\left(\phi,|u(t)|^{2} u(t)\right) .
$$

Let $X=C\left([0, T], H^{1,1}\left(\mathbb{R}^{+}\right)\right), 0<T<\infty$, denote the set of continuous maps from $[0, T]$ to $H^{1,1}\left(\mathbb{R}^{+}\right)$. Equip $\mathrm{X}$ with a norm, $\|v\|_{X} \equiv$ $\sup _{0 \leq t \leq T}\|v(t)\|_{H^{1,1}\left(\mathbb{R}^{+}\right)}$. For $v=v(t) \in X$, define an operator

$$
\left(T_{q} v\right)(t)=U_{t} v(0)+i \int_{0}^{t} U_{t-s}|v(s)|^{2} v(s) \mathrm{d} s, \quad 0 \leq t \leq T .
$$

Step 1 (Local existence and uniqueness): $T_{q}$ is a locally Lipschitz continuous mapping from $X$ to itself.

From standard spectral theory(see CL55]), we have

$$
\begin{aligned}
U_{t} w=\frac{1}{2 \pi} & \int_{0}^{\infty} e^{-i z^{2} t / 2}\left(f_{q}, w\right)(z) f_{q}(x, z) \mathrm{d} z \\
& +2 q\left(\int_{0}^{\infty} e^{-q y} w(y) \mathrm{d} y\right) e^{-q x+i q^{2} t / 2}, \quad w \in L^{2}\left(\mathbb{R}^{+}\right),
\end{aligned}
$$


for $q>0$ where

$$
f_{q}(x, z)=e^{i x z}-\frac{q+i z}{q-i z} e^{-i x z} .
$$

If $q<0$, the second term is omitted in the spectral representation (2.2). As $U_{t}$ is unitary in $L^{2}\left(\mathbb{R}^{+}\right),\left\|U_{t} w\right\|_{L^{2}\left(\mathbb{R}^{+}\right)}=\|w\|_{L^{2}\left(\mathbb{R}^{+}\right)}$. Now for $w \in H^{1,1}\left(\mathbb{R}^{+}\right)$define

$$
w_{q}(x) \equiv\left\{\begin{array}{l}
w^{\prime}(x)+q w(x), \quad x \geq 0, \\
-\left(w^{\prime}(-x)+q w(-x)\right), \quad x<0,
\end{array}\right.
$$

Then, a direct calculation shows that

$$
\left(U_{t} w\right)_{q}=e^{-i H_{0} t / 2} w_{q} .
$$

As $e^{-i H_{0} t / 2}$ is unitary in $L^{2}(\mathbb{R})$, it immediately follows that $\left\|\left(U_{t} w\right)^{\prime}\right\|_{L^{2}\left(\mathbb{R}^{+}\right)} \leq$ $c_{1}\|w\|_{H^{1,1}\left(\mathbb{R}^{+}\right)}$. Moreover, by using integration by parts, we obtain $\left\|x\left(U_{t} w\right)\right\|_{L^{2}\left(\mathbb{R}^{+}\right)} \leq\left(c_{2} t+c_{3}\right)\|w\|_{H^{1,1}\left(\mathbb{R}^{+}\right)}$. Assembling the above results, we see that $\left\|U_{t} w\right\|_{H^{1,1}\left(\mathbb{R}^{+}\right)} \leq\left(c_{4} t+c_{5}\right)\|w\|_{H^{1,1}\left(\mathbb{R}^{+}\right)}$and hence for $v \in X$,

$$
\begin{aligned}
&\left\|\left(T_{q} v\right)(t)\right\|_{H^{1,1}\left(\mathbb{R}^{+}\right)} \\
& \leq\left\|U_{t} v(0)\right\|_{H^{1,1}\left(\mathbb{R}^{+}\right)}+\int_{0}^{t}\left\|U_{t-s}|v(s)|^{2} v(s)\right\|_{H^{1,1}\left(\mathbb{R}^{+}\right)} \mathrm{d} s \\
& \quad \leq\left(c_{4} t+c_{5}\right)\left(\|v(0)\|_{H^{1,1}\left(\mathbb{R}^{+}\right)}+\int_{0}^{t}\left\||v(s)|^{2} v(s)\right\|_{H^{1,1}\left(\mathbb{R}^{+}\right)} \mathrm{d} s\right) .
\end{aligned}
$$

Thus, $\left\|T_{q} v\right\|_{X} \leq\left(c_{4} T+c_{5}\right)\left(1+c_{6} T\|v\|_{X}^{2}\right)\|v\|_{X}<\infty$. For $v_{1}, v_{2} \in X$,

$$
\begin{aligned}
\| T_{q} v_{1} & -T_{q} v_{2} \|_{X} \\
& \leq \sup _{0 \leq t \leq T} \int_{0}^{t}\left\|U_{t-s}\left(\left|v_{1}(s)\right|^{2} v_{1}(s)-\left|v_{2}(s)\right|^{2} v_{2}(s)\right)\right\|_{H^{1,1}\left(\mathbb{R}^{+}\right)} \mathrm{d} s \\
& \leq c_{7} T\left(c_{4} T+c_{5}\right) \sup _{0 \leq t \leq T}\left(\left\|v_{1}\right\|_{H^{1,0}}^{2}+\left\|v_{2}\right\|_{H^{1,0}}^{2}\right)\left\|v_{1}-v_{2}\right\|_{X},
\end{aligned}
$$

where $H^{1,0}=\left\{w \in L^{2}: w\right.$ is absolutely continuous $\left.w^{\prime} \in L^{2}\right\}$ is the first Sobolev space. This proves Step 1. But then, it follows by a standard fixed point argument that there exists a unique (weak) solution to HNLS $_{q}^{+}$locally in $H^{1,1}\left(\mathbb{R}^{+}\right)$. Let $u(t)=u(x, t), 0 \leq t \leq T$, be the solution to $\operatorname{HNLS}_{q}^{+}$with $u(t=0)=u_{0} \in H^{1,1}\left(\mathbb{R}^{+}\right)$.

Step 2: If $u_{0} \in H^{1,1}\left(\mathbb{R}^{+}\right)$and $u_{0}^{\prime} \in L^{1}\left(\mathbb{R}^{+}\right)$, then $u_{x}(x, t)$ is continuous in $x \in \mathbb{R}^{+}$and the boundary condition $u_{x}(0+, t)+q u(0, t)=0$ holds for all $t>0$. Moreover,

$$
\left|u_{x}(x, t)\right| \leq \frac{c}{\sqrt{t}}, \quad 0<t \leq T, 0<T<\infty .
$$


From (1.9) and (2.3), we have

$$
(u(t))_{q}=e^{-i H_{0} t / 2}\left(u_{0}\right)_{q}+i \int_{0}^{t} e^{-i H_{0}(t-s) / 2}\left(|u(s)|^{2} u(s)\right)_{q} \mathrm{~d} s .
$$

For $w \in H^{1,1}\left(\mathbb{R}^{+}\right)$such that $w^{\prime} \in L^{1}\left(\mathbb{R}^{+}\right)$, observe that $w_{q} \in L^{1}(\mathbb{R})$. Using the classical formula for the kernel of $e^{-i H_{0} t / 2}$, we obtain from (2.3),

$$
\left(U_{t} w\right)_{q}=\sqrt{\frac{2}{\pi t}} \int_{\mathbb{R}} w_{q}(s+x) e^{i\left(\frac{s^{2}}{2 t}-\frac{\pi}{4}\right)} \mathrm{d} s, \quad t>0 .
$$

Hence, $\left(U_{t} w\right)_{q}$ is continuous in $x$ and $\left|\left(U_{t} w\right)_{q}\right| \leq \frac{c}{\sqrt{t}}\left\|w_{q}\right\|_{L^{1}\left(\mathbb{R}^{+}\right)}$. Setting $w=u_{0}$ in (2.7), we see that the first term in (2.6) is continuous in $x$. Clearly $|u(t)|^{2} u(t) \in H^{1,1}\left(\mathbb{R}^{+}\right) \subset L^{1}\left(\mathbb{R}^{+}\right)$and $\left(|u(t)|^{2} u(t)\right)^{\prime} \in L^{1}\left(\mathbb{R}^{+}\right)$. As $\frac{1}{\sqrt{t-s}}$ is integrable on $[0, t], 0 \leq t \leq T$, the second term in (2.6) is also continuous in $x$. Thus, $(u(x, t))_{q}$ is continuous and, as $u(x, t)$ is clearly continuous, we conclude that $u_{x}(x, t)$ continuous. As $w_{q}$ is odd, it follows from (2.7) that $\left(U_{t} w\right)_{q}(x=0)=0$ and hence the boundary condition $u_{x}(0+, t)+q u(0+, t)=0,0<t \leq T$, immediately follows from (2.6).

Step 3 (Conserved quantities): $\mathcal{M} \equiv \int_{0}^{\infty}|u|^{2} \mathrm{~d} x$ and $\mathcal{H} \equiv \int_{0}^{\infty}\left|u_{x}\right|^{2}-$ $|u|^{4} \mathrm{~d} x-q|u(0)|^{2}$ are constant in $t$.

The usual approach here is to prove by direct calculation that $\mathcal{M}$ and $\mathcal{H}$ are conserved for classical solutions (with smooth initial data), and then to use an approximation argument. However, the existence of classical solutions for $\mathrm{HNLS}_{q}^{+}$does not appear to be an elementary matter. The technical issue is that if a smooth function $u$ satisfies the boundary condition $u^{\prime}(0)+q u(0)=0$, then $|u|^{2} u$ in general does not. In other words, although $|u|^{2} u$ is smooth, $|u|^{2} u$ may not be in the domain of the operator $\mathrm{H}_{q}^{+}$and so an iteration in a higher Sobolev space fails.

On the other hand, classical solutions to (1.9) satisfying the boundary conditions, with arbitrary orders of smoothness and decay, can indeed be shown to exist (see Remark 4.34 below). The proof of the existence of such smooth solutions, relies ultimately on Riemann-Hilbert methods. (cf. proof of Proposition 4.34) Such solutions could be used, in particular, to verify Step 3 for general $u \in H^{1,1}\left(\mathbb{R}^{+}\right)$by approximation as just described. However, we will present a direct proof of Step 3 which does not use approximation by smooth solutions. Relying on Riemann-Hilbert techniques to prove the existence of global solutions of (1.9) does not seem appropriate. Even more to the point, similar 
issues arise in the analysis of (1.8). In this case, solutions to the equation which are smooth enough to yield a direct proof of the constancy of $\mathcal{M}$ and $\mathcal{H}$, are not even known to exist for general (non-even) data (cf. Step 5 below). So one needs the analog for (1.8) of the proof given below, to prove global existence.

Suppose first that $u_{0} \in H^{1,1}\left(\mathbb{R}^{+}\right)$and $u_{0}^{\prime} \in L^{1}\left(\mathbb{R}^{+}\right)$. As $U_{t}$ is unitary, we have for $\breve{u}(t)=U_{-t} u(t)$,

$$
\begin{aligned}
\frac{d}{d t} \int_{0}^{\infty}|u|^{2} \mathrm{~d} x & =\frac{d}{d t} \int_{0}^{\infty}|\breve{u}|^{2} \mathrm{~d} x=\int_{0}^{\infty} \breve{u}_{t} \overline{\breve{u}}+\breve{u} \overline{\breve{u}_{t}} \mathrm{~d} x \\
& =\int_{0}^{\infty}\left(i U_{-t}|u|^{2} u\right) \overline{\breve{u}}+\breve{u}\left(\overline{i U_{-t}|u|^{2} u}\right) \mathrm{d} x \\
& =\int_{0}^{\infty}\left(i|u|^{2} u\right) \bar{u}+u\left(\overline{\left(i|u|^{2} u\right.}\right) \mathrm{d} x=0
\end{aligned}
$$

and hence $\int_{0}^{\infty}|u|^{2} \mathrm{~d} x$ is constant. For $\mathcal{H}$, we first have

$$
\begin{aligned}
\int_{0}^{\infty}\left|u_{q}\right|^{2} \mathrm{~d} x & =\int_{0}^{\infty}\left|u_{x}+q x\right|^{2} \mathrm{~d} x \\
& =\int_{0}^{\infty}\left|u_{x}\right|^{2} \mathrm{~d} x-q|u(0, t)|^{2}+q^{2} \int_{0}^{\infty}|u|^{2} \mathrm{~d} x
\end{aligned}
$$

On the other hand, again as $e^{i H_{0} t / 2}$ is unitary in $L^{2}(\mathbb{R})$, it follows from (2.6) and the oddness of $u_{q}(x)$ that

$$
\begin{aligned}
\frac{d}{d t} & \int_{0}^{\infty}\left|u_{q}\right|^{2} \mathrm{~d} x \\
& =\frac{1}{2} \frac{d}{d t} \int_{\mathbb{R}}\left|u_{q}\right|^{2} \mathrm{~d} x=\frac{1}{2} \frac{d}{d t} \int_{\mathbb{R}}\left|e^{i H_{0} t / 2} u_{q}\right|^{2} \mathrm{~d} x \\
& \left.=\frac{1}{2} \int_{\mathbb{R}} \overline{e^{i H_{0} t / 2} u_{q}}\left(e^{i H_{0} t / 2} u_{q}\right)_{t}+e^{i H_{0} t / 2} u_{q} \overline{\left(e^{i H_{0} t / 2} u_{q}\right.}\right)_{t} \mathrm{~d} x \\
& \left.=\frac{1}{2} \int_{\mathbb{R}} \overline{e^{i H_{0} t / 2} u_{q}}\left(i e^{i H_{0} t / 2}\left(|u|^{2} u\right)_{q}\right)+e^{i H_{0} t / 2} u_{q} \overline{\left(e^{i H_{0} t / 2}\left(|u|^{2} u\right)_{q}\right.}\right) \mathrm{d} x \\
& =\frac{i}{2} \int_{\mathbb{R}} \overline{u_{q}}\left(|u|^{2} u\right)_{q}-u_{q}\left(|u|^{2} \bar{u}\right)_{q} \mathrm{~d} x \\
& =i \int_{0}^{\infty}\left(\overline{u_{x}+q u}\right)\left(\left(|u|^{2} u\right)^{\prime}+q|u|^{2} u\right) \\
& =i \int_{0}^{\infty} u^{2} \bar{u}_{x}^{2}-\bar{u}^{2} u_{x}^{2} \mathrm{~d} x \equiv D(t)
\end{aligned}
$$


Hence, by (2.8) together with the fact that $\int_{0}^{\infty}|u|^{2}$ is constant,

$$
\frac{d}{d t}\left(\int_{0}^{\infty}\left|u_{x}\right|^{2} \mathrm{~d} x-q|u(0, t)|^{2}\right)=D(t)
$$

Let $j(x)$ be any $C^{\infty}$-function supported on $(-1,1)$ such that $j \geq 0$ and $\int_{\mathbb{R}} j(x) \mathrm{d} x=1$, and set $j_{\epsilon}(x) \equiv \frac{1}{\epsilon} j\left(\frac{x}{\epsilon}\right)$. For any function $w \in H^{1,1}\left(\mathbb{R}^{+}\right)$, denote $w_{\epsilon}(x) \equiv \int_{0}^{\infty} j_{\epsilon}(x-y) w(y) \mathrm{d} y$. Note that $j_{\epsilon}(x-y) \in C_{0}^{\infty}(0<$ $y<\infty)$ for $x>\epsilon$. Hence from (2.1), we have for $x>\epsilon$,

$$
\left(u_{\epsilon}\right)_{t}=\frac{i}{2}\left(u_{\epsilon}\right)_{x x}+i\left(|u|^{2} u\right)_{\epsilon} .
$$

Thus, integrating by parts and noting that $\left(u_{\epsilon}\right)_{x}=\left(u_{x}\right)_{\epsilon}$ for $x>\epsilon$, we obtain

$$
\begin{aligned}
\frac{d}{d t} \int_{\epsilon}^{\infty}\left|u_{\epsilon}\right|^{4} & =2 \int_{\epsilon}^{\infty} \bar{u}_{\epsilon}\left(\bar{u}_{\epsilon}\right)_{t} u_{\epsilon}^{2}+\bar{u}_{\epsilon}^{2} u_{\epsilon}\left(u_{\epsilon}\right)_{t} \\
& =D_{\epsilon}(t)+\delta_{1}^{\epsilon}(t)+\delta_{2}^{\epsilon}(t),
\end{aligned}
$$

where

$$
\begin{aligned}
& D_{\epsilon}(t)=i \int_{\epsilon}^{\infty} u_{\epsilon}^{2}\left(\bar{u}_{\epsilon}\right)_{x}^{2}-\bar{u}_{\epsilon}^{2}\left(u_{\epsilon}\right)_{x}^{2}, \\
& \delta_{1}^{\epsilon}(t)=i\left(u_{\epsilon}^{2} \bar{u}_{\epsilon}\left(\bar{u}_{\epsilon}\right)_{x}-\bar{u}_{\epsilon}^{2} u_{\epsilon}\left(u_{\epsilon}\right)_{x}\right)(\epsilon), \text { and } \\
& \delta_{2}^{\epsilon}(t)=2 i \int_{\epsilon}^{\infty} \bar{u}_{\epsilon}^{2} u_{\epsilon}\left(|u|^{2} u\right)_{\epsilon}-\bar{u}_{\epsilon} u_{\epsilon}^{2}\left(|u|^{2} \bar{u}\right)_{\epsilon} .
\end{aligned}
$$

As $j_{\epsilon}$ is an approximate identity, we see that for each $0<t \leq T \delta_{1}^{\epsilon}(t)$ and $\delta_{2}^{\epsilon}(t) \rightarrow 0$ as $\epsilon \downarrow 0$ : here we need the fact that $u_{x}(x, t)$ is continuous in $x$ and satisfies the boundary condition $u_{x}(0+, t)+q u(0, t)=0$. Also $D_{\epsilon}(t) \rightarrow D(t)$. Moreover, it follows from (2.5), and the fact that $t \mapsto u(t)$ is continuous, and hence bounded for $0<t \leq T$, that $D_{\epsilon}(t)+\delta_{1}^{\epsilon}(t)+\delta_{2}^{\epsilon}(t)$ is bounded by $\frac{c}{\sqrt{t}}$, uniformly for $\epsilon>0$. Thus from (2.9) and (2.10), we obtain

$$
\begin{gathered}
\int_{0}^{\infty}\left|u_{x}(x, t)\right|^{2} \mathrm{~d} x-q|u(0, t)|^{2}-\int_{\epsilon}^{\infty}|u(x, t)|^{4} \mathrm{~d} x \\
=\int_{0}^{\infty}\left|u_{x}(x, 0)\right|^{2} \mathrm{~d} x-q|u(0,0)|^{2}-\int_{\epsilon}^{\infty}|u(x, 0)|^{4} \mathrm{~d} x \\
+\int_{0}^{t} D(s)-D_{\epsilon}(s)-\delta_{1}^{\epsilon}(s)-\delta_{2}^{\epsilon}(s) \mathrm{d} s .
\end{gathered}
$$

Now it follows by dominated convergence theorem that $\mathcal{H}$ is constant.

Step 3 for general data $u_{0} \in H^{1,1}\left(\mathbb{R}^{+}\right)$, then follows by approximation, $u_{0} \rightarrow \check{u}_{0}=u_{0} \chi_{R}, R \rightarrow \infty$, where $\chi_{R} \in C_{0}^{\infty}[0, \infty), \chi_{R}(x)=1$ for $0 \leq x \leq R$, and $\chi_{R}(x)=0$ for $x>R+1$. 
Step 4 (Global existence: The solution $u(x, t)$ of $\mathrm{HNLS}_{q}^{+}$in $H^{1,1}\left(\mathbb{R}^{+}\right.$) exists globally for all $t \geq 0$.

From the basic estimates, we have for any constant $M>0$,

$$
\|u\|_{L^{\infty}\left(\mathbb{R}^{+}\right)}^{2} \leq 2\|u\|_{L^{2}\left(\mathbb{R}^{+}\right)}\left\|u_{x}\right\|_{L^{2}\left(\mathbb{R}^{+}\right)} \leq M \mathcal{M}+\frac{1}{M}\left\|u_{x}\right\|_{L^{2}\left(\mathbb{R}^{+}\right)}^{2},
$$

and hence

$$
\begin{aligned}
\left\|u_{x}\right\|_{L^{2}\left(\mathbb{R}^{+}\right)}^{2} & =\left.\left|\mathcal{H}+\int_{0}^{\infty}\right| u\right|^{4}+q|u(0)|^{2} \mid \\
& \leq|\mathcal{H}|+\|u\|_{L^{\infty}\left(\mathbb{R}^{+}\right)}^{2}(\mathcal{M}+|q|) \\
& \leq|\mathcal{H}|+\left(M \mathcal{M}+\frac{1}{M}\left\|u_{x}\right\|_{L^{2}\left(\mathbb{R}^{+}\right)}^{2}\right)(\mathcal{M}+|q|) .
\end{aligned}
$$

Thus, if we set $M=2(\mathcal{M}+|q|)$, then, as $\mathcal{M}$ and $\mathcal{H}$ are constant, $\left\|u_{x}\right\|_{L^{2}\left(\mathbb{R}^{+}\right)}$is uniformly bounded.

As Lipschitz constant in (2.4) depends only on the $H^{1,0}$-norm of $u(t)$, it follows by standard arguments using the a priori bound on $\left\|u_{x}(x, t)\right\|_{L^{2}},\|u(x, t)\|_{L^{2}}$ that the solution of $\mathrm{HNLS}_{q}^{+}$exists globally.

Step 5: Set $\hat{u}_{0}(x) \equiv u_{0}(|x|)$ and $\hat{u}(x, t) \equiv u(|x|, t), x \in \mathbb{R}$. Then, $\hat{u}(x, t)$ solves (1.8) with $\hat{u}(t=0)=\hat{u}_{0}$.

The proof follows immediately by applying the following result,

$$
\left(e^{-i H_{q} t / 2} \hat{w}\right)(x)=\left(e^{-i H_{q}^{+} t / 2} w\right)(x), \quad x>0
$$

for any $w \in L^{2}\left(\mathbb{R}^{+}\right)$, and $\hat{w}(x)=w(|x|)$. This identity follows directly by using the spectral representation (2.2) for $e^{-i H_{q}^{+} t / 2}$ and the spectral representation (cf. [CL55])

$$
\begin{aligned}
& \left(e^{-i H_{q} t / 2} \hat{w}\right)(x) \\
& =\frac{1}{4 \pi} \int_{\mathbb{R}} e^{-i z^{2} t / 2} \frac{z^{2}}{z^{2}+q^{2}}\left[\left(f_{1}, \hat{w}\right)(z) f_{1}(x, z)+\left(f_{2}, \hat{w}\right)(z) f_{2}(x, z)\right] \mathrm{d} z \\
& \quad+q\left(\int_{\mathbb{R}} e^{-q|y|} \hat{w}(y) \mathrm{d} y\right) e^{-q|x|}
\end{aligned}
$$

in the case $q>0$, where $f_{1}(x, z)=e^{i x z}, x>0$, and $f_{1}(x, z)=(1+$ $\left.\frac{q}{i z}\right) e^{i x z}-\frac{q}{i z} e^{-i x z}, x<0$, and $f_{2}(x, z)=f_{1}(-x, z)$. In the case $q<0$, the second term is omitted.

For NLS on the line, solutions to IVP exist globally in $H^{k, k}(\mathbb{R})$ for all $k \geq 1$.

Proposition 2.1. Let $u_{0} \in H^{k, k}(\mathbb{R}), k \geq 1$. Then $N L S$ on $\mathbb{R}$ has a (unique) solution with $u(t=0)=u_{0}$ in $H^{k, k}(\mathbb{R})$, i.e., there exists a 
continuous map $t \mapsto u(t) \in H^{k, k}(\mathbb{R}) \subset H^{1,1}(\mathbb{R}), u(t=0)=u_{0}$ such that $u(t)$ solves (1.10).

Remark 2.2. A standard argument shows that if $u(t)$ solves NLS in $H^{k, k}(\mathbb{R})$ for $k \geq 3$, then $u(t)$ is a classical solution.

\section{Scattering And Inverse Scattering}

We now recall the Riemann-Hilbert version (see [BC84] Zho89] [Zho98]; see also [DZ91] BDT88]) of scattering and inverse scattering for the focusing NLS equation on the line,

$$
\begin{aligned}
& i u_{t}+\frac{1}{2} u_{x x}+|u|^{2} u=0, \quad t \geq 0, \quad-\infty<x<\infty, \\
& u(x, t=0)=u_{0}(x) .
\end{aligned}
$$

Let $L$ denote the Zakharov-Shabat Lax operator in (1.28) above and set

$$
E=-\frac{i}{2} z^{2} \sigma-\frac{1}{2} z\left(\begin{array}{cc}
0 & u \\
-\bar{u} & 0
\end{array}\right)+\frac{1}{2}\left(\begin{array}{cc}
i|u|^{2} & i u_{x} \\
i \bar{u}_{x} & -i|u|^{2}
\end{array}\right) .
$$

The operators $\partial_{x}-L$ and $\partial_{t}-E$ form a Lax pair for the focusing NLS equation in the sense that the compatibility of $\partial_{x} \psi=L \psi$ and $\partial_{t} \psi=E \psi$, i.e. $\partial_{t}\left(\partial_{x} \psi\right)=\partial_{x}\left(\partial_{t} \psi\right)$ is equivalent to NLS (3.1), i.e., $u=u(x, t)$ is a (classical) solution to (3.1) if and only if $\partial_{x}-L$ and $\partial_{t}-E$ commute as operators on smooth functions $\psi=\psi(x, t)$, i.e.,

$$
\left(\partial_{x}-L\right)\left(\partial_{t}-E\right)=\left(\partial_{t}-E\right)\left(\partial_{x}-L\right) .
$$

Alternatively, NLS is equivalent to an iso-spectral deformation of the operator

$$
T=T(u)=(i \sigma)^{-1}\left(\partial_{x}-\left(\begin{array}{cc}
0 & u(x) \\
-u(x) & 0
\end{array}\right)\right) .
$$

The following facts about solutions $\psi$ of $T \psi=z \psi$, or equivalently,

$$
\psi_{x}=L \psi=(i z \sigma+Q) \psi, \quad Q(x)=\left(\begin{array}{cc}
0 & u(x) \\
-u(x) & 0
\end{array}\right),
$$

play a basic role in the theory that follows. The proofs are direct calculations. Let $\sigma_{2}=\left(\begin{array}{cc}0 & -i \\ i & 0\end{array}\right)$ be the second Pauli matrix.

Proposition 3.1. Let $\psi(x, z)$ solve (3.5) $)$. Then,

(i) $\overline{\sigma_{2} \psi(x, \bar{z}) \sigma_{2}}$ also solves (3.5) and

(ii) $\sigma_{3} \psi(-x,-z) \sigma_{3}$ solves (3.5) with $Q(x)$ replaced by $Q(-x)$. 
Of course the factors $\sigma_{2}$ and $\sigma_{3}$ on the right in (i),(ii) can be omitted: we include them here in view of the applications that follow.

The scattering map $\mathcal{S}$ (see Definition 3.7 below) maps $T$ to its scattering data. We now describe the properties of $\mathcal{S}$ when $u$ lies in the Sobolev space $H^{1,1}=\left\{u \in L^{2}(\mathbb{R}): u^{\prime}(x), x u(x) \in L^{2}(\mathbb{R})\right\}$. This is sufficient for our purpose as we will only consider NLS and (1.4) in $H^{1,1}$. (cf. [DZ03]) (For the action of $\mathcal{S}$ on the other spaces e.g. $H^{k, j}=\left\{u \in L^{2}(\mathbb{R}): u, u^{\prime}, \cdots, u^{(k-1)}\right.$ are absolutely continuous, $\left.u^{(k)}(x), x^{j} u(x) \in L^{2}(\mathbb{R})\right\}, k \geq 0, j \geq 1$, or Schwartz space, see [Zho98]).

We use the standard notation $\mathbb{C}^{+}=\{\operatorname{Im} z>0\}, \mathbb{C}^{-}=\{\operatorname{Im} z<0\}$, $e_{1}=(1,0)^{T}$ and $e_{2}=(0,1)^{T}$. For $u \in H^{1,1}$ and $z \in \mathbb{C}^{+}$, the equation

$$
\phi_{x}=(i z \sigma+Q) \phi
$$

has a unique column solution $\phi=\left(\phi_{1}, \phi_{2}\right)^{T}$ such that

$$
\phi(x, z) e^{i x z / 2} \rightarrow e_{2} \text { as } x \rightarrow-\infty .
$$

As $x \rightarrow \infty$,

$$
\phi(x, z) e^{i x z / 2} \rightarrow a(z) e_{2} .
$$

where the scattering function $a(z)$ is

- analytic in $\mathbb{C}^{+}$,

- continuous in $\overline{\mathbb{C}^{+}}$and

- $a(z) \rightarrow 1$ as $z \rightarrow \infty$ in $\overline{\mathbb{C}^{+}}$.

We say that $u \in H^{1,1}(\mathbb{R})$ is generic if $a(z)$ is non-zero in $\overline{\mathbb{C}^{+}}$except at a finite number of points $z_{1}, \cdots, z_{n} \in \mathbb{C}^{+}$where it has simple zeros $a\left(z_{k}\right)=0, a^{\prime}\left(z_{k}\right) \neq 0, k=1, \cdots, n$. The set of generic functions $u(x)$ is an open dense subset of $H^{1,1}(\mathbb{R})$ (see Zho98 BDT88]), which we denote by $\mathcal{G}$. Set $Z_{+}=\left\{z_{1}, \cdots, z_{n}\right\}, Z_{-}=\left\{\overline{z_{1}}, \cdots, \overline{z_{n}}\right\}$, and $Z=Z_{+} \cup Z_{-}$.

Theorem 4 (Direct Scattering: see [BC84] Zho98]; also cf. [BDT88]). Let $u \in \mathcal{G} \subset H^{1,1}$.

(1) For $z \in \mathbb{C} \backslash(\mathbb{R} \cup Z)$, the equation $T \psi=z \psi$ has a unique solution $\psi=\psi(x, z)$ such that

(i) $\psi(x, z) e^{-i x z \sigma}$ is bounded for $x \in \mathbb{R}$ and

(ii) $\psi(x, z) e^{-i x z \sigma} \rightarrow I$ as $x \rightarrow-\infty$.

(2) Points $z \in Z$ correspond to $L^{2}(\mathbb{R})$ eigenvalues of $T$, $T h=z h$, $h=\left(h_{1}, h_{2}\right)^{T} \in L^{2}(\mathbb{R})$. Moreover, $z=z_{k}$ is an $L^{2}$ eigenvalue if and only if $z=\overline{z_{k}}$ is an $L^{2}$ eigenvalue. The associated eigenfunctions $h$ have asymptotics

$$
\begin{aligned}
h=h\left(x, z_{k}\right) & =\left(e_{1}+o(1)\right) e^{i x z_{k} / 2} \quad \text { as } x \rightarrow+\infty, \\
& =\gamma\left(z_{k}\right)\left(e_{2}+o(1)\right) e^{-i x z_{k} / 2} \quad \text { as } x \rightarrow-\infty,
\end{aligned}
$$


and

$$
\begin{aligned}
h=h\left(x, \overline{z_{k}}\right) & =\left(e_{2}+o(1)\right) e^{-i x \overline{z_{k}} / 2} \quad \text { as } x \rightarrow+\infty, \\
& =\gamma\left(\overline{z_{k}}\right)\left(e_{1}+o(1)\right) e^{i x \overline{z_{k}} / 2} \quad \text { as } x \rightarrow-\infty,
\end{aligned}
$$

where $\gamma\left(z_{k}\right)=-\overline{\gamma\left(\overline{z_{k}}\right)} \neq 0$.

(3) For all $x \in \mathbb{R}$, the solution $\psi(x, z)$ in (1)

- is analytic in $\mathbb{C} \backslash(\mathbb{R} \cup Z)$,

- has simple poles at $Z$ such that

$$
\begin{aligned}
& \operatorname{Res}_{z=z_{k}} \psi(x, z)=\lim _{z \rightarrow z_{k}} \psi(x, z)\left(\begin{array}{cc}
0 & 0 \\
c\left(z_{k}\right) & 0
\end{array}\right), \\
& \operatorname{Res}_{z=\overline{z_{k}}} \psi(x, z)=\lim _{z \rightarrow \overline{z_{k}}} \psi(x, z)\left(\begin{array}{cc}
0 & c\left(\overline{z_{k}}\right) \\
0 & 0
\end{array}\right) .
\end{aligned}
$$

where $c\left(z_{k}\right)=\gamma\left(z_{k}\right) / a^{\prime}\left(z_{k}\right), c\left(\overline{z_{k}}\right)=-\overline{c\left(z_{k}\right)}, k=1, \cdots, n$, and

- has continuous boundary values $\psi_{ \pm}(x, z)=\lim _{\epsilon \downarrow 0} \psi(x, z \pm i \epsilon)$, $z \in \mathbb{R}$, satisfying the jump relation

$$
\psi_{+}(x, z)=\psi_{-}(x, z) v(z), \quad z \in \mathbb{R},
$$

where the jump matrix $v(z)$ has the form

$$
v(z)=\left(\begin{array}{cc}
1+\frac{|r(z)|^{2}}{r(z)} & r(z) \\
1
\end{array}\right)
$$

and the reflection coefficient $r(z) \in H^{1,1}$.

(4) For all $x \in \mathbb{R}$,

- $\psi(x, z) e^{-i x z \sigma} \rightarrow I$ as $z \rightarrow \infty$ in $\mathbb{C}^{+}$or in $\mathbb{C}^{-}$.

- $\psi(x, z) e^{-i x z \sigma}=I+\frac{m_{1}(x)}{z}+o\left(\frac{1}{z}\right)$ as $z \rightarrow \infty$ in any cone $|\operatorname{Im} z|>c|\operatorname{Re} z|, c>0$.

Remark 3.2. A solution $\psi$ of $T \psi=z \psi, z \in \mathbb{C} \backslash \mathbb{R}$, satisfying (1)(i)(ii) is known as a Beals-Coifman solution. For emphasis we denote $\psi=\psi_{B C}$.

Remark 3.3. Note that as the vector field in (3.6) has trace zero, $\operatorname{det} \psi(x, z)$ is independent of $x$. Hence by $(1)(\mathrm{i})$,

$$
\operatorname{det} \psi(x, z)=1 \text {, for all } x \in \mathbb{R}, z \in \mathbb{C} \backslash(\mathbb{R} \cup Z) \text {. }
$$

Remark 3.4. If $u \in H^{1,1}(\mathbb{R})$ is not generic, the zero set of $a(z ; u)$ can be extremely complicated. For example see [Zho89]. Let $z_{0}>0$ be an arbitrary positive number and let $\epsilon>0$ be small. Let $D^{+}=\left\{z \in \mathbb{C}^{+}\right.$: $\left.\left|z-z_{0}\right|<\epsilon\right\}$. Let $a(z)$ be any function which is analytic in $D^{+}$, continuous up to the boundary $\partial D^{+}$, and non-zero in $\partial D^{+} \cap \mathbb{C}^{+}$. Then there exists $u$ in Schwartz space, $\mathcal{S}(\mathbb{R})$, such that $\Delta=\left\{z \in D^{+}: a(z)=0\right\} \subset$ 
$\operatorname{spec}\{T(u)\}$. Thus we see, in particular, that there exist a Schwartz function $u$ with $L^{2}(\mathbb{R})$-eigenvalues $\left\{z_{k}\right\} \subset \mathbb{C}^{+}$accumulating at essentially arbitrary rates onto the real axis. The long time asymptotics of solutions of NLS with such initial data $u$, is correspondingly extremely complicated and not yet fully understood. This is in sharp contrast to the case where $u \in \mathcal{G}$ (see below).

We note that the second column of $\psi(x, z), z \in \mathbb{C}^{+}$, is in fact the solution $\phi=\left(\phi_{1}, \phi_{2}\right)^{T}$ in (3.6) (3.7) above. Define

$$
a(z) \equiv \overline{a(\bar{z})}, \quad z \in \mathbb{C}^{-} \text {. }
$$

The asymptotic behavior of $\psi(x, z) e^{-i x z \sigma}$ in (1)(i)(ii) above is in $H^{1,1}$ in the following sense.

Proposition 3.5. Let $z \in \mathbb{C} \backslash(\mathbb{R} \cup Z)$. Let $\chi^{+}(x)$ be a $C^{\infty}$ function such that $0 \leq \chi^{+} \leq 1, \chi^{+}(x)=1$ for $x \geq 1$ and $\chi^{+}(x)=0$ for $x \leq 0$. Let $\chi^{-}=1-\chi^{+}$. Then,

$$
\begin{aligned}
& \left\|\left(\psi(x, z) e^{-i x z \sigma}-I\right) \chi^{-}\right\|_{H^{1,1}} \leq c(u), \\
& \left.\|\left(\psi(x, z) e^{-i x z \sigma}-a(z)^{-\sigma_{3}}\right) \chi^{+}\right) \|_{H^{1,1}} \leq c(u),
\end{aligned}
$$

Proof. Let $z \in \mathbb{C}^{+} \backslash Z$. The other case $z \in \mathbb{C}^{-} \backslash Z$ is similar. Let $m(x, z)=\left(m_{1}, m_{2}\right)=\psi(x, z) e^{-i x z \sigma}$. Then (cf. [BC84], [Zho98]), as $a(z) \neq 0, m_{1}, m_{2}$ are obtained as solutions of the following Volterra integral equations

$$
m_{1}(x, z)=a(z)^{-1} e_{1}-\int_{x}^{\infty}\left(\begin{array}{cc}
1 & 0 \\
0 & e^{i z(y-x)}
\end{array}\right)\left(\begin{array}{cc}
0 & u \\
-\bar{u} & 0
\end{array}\right) m_{1}(y, z) \mathrm{d} y
$$

and

$$
m_{2}(x, z)=e_{2}+\int_{-\infty}^{x}\left(\begin{array}{cc}
e^{-i z(y-x)} & 0 \\
0 & 1
\end{array}\right)\left(\begin{array}{cc}
0 & u \\
-\bar{u} & 0
\end{array}\right) m_{2}(y, z) \mathrm{d} y
$$

We consider (3.12): the analysis of (3.11) is similar. The solution $m_{2}(x, z)$ of (3.12) is obtained by standard iterations, which yields the bound

$$
\sup _{x \in \mathbb{R}}\left|m_{2}(x, z)\right| \leq e^{\int_{-\infty}^{\infty}|u(y)| \mathrm{d} y} .
$$

Writing $m_{2}=\left(\left(m_{2}\right)_{1},\left(m_{2}\right)_{2}\right)^{T}$, (3.12) becomes

$$
\left(m_{2}\right)_{1}(x, z)=\int_{-\infty}^{x} e^{-i z(y-x)} u(y)\left(m_{2}\right)_{2}(y, z) \mathrm{d} y
$$

and

$$
\left(m_{2}\right)_{2}(x, z)=1-\int_{-\infty}^{x} \bar{u}(y)\left(m_{2}\right)_{1}(y, z) \mathrm{d} y
$$


From Lemma 3.6 below,

$$
\left\|\left(m_{2}\right)_{1}\right\|_{H^{1,1}(\mathbb{R})} \leq c\left\|u\left(m_{2}\right)_{2}\right\|_{H^{0,1}(\mathbb{R})} \leq c\left\|\left(m_{2}\right)_{2}\right\|_{L^{\infty}(\mathbb{R})}\|u\|_{H^{0,1}(\mathbb{R})},
$$

and hence from Lemma 3.6 again,

$$
\left\|\left(\left(m_{2}\right)_{2}-1\right) \chi^{-}\right\|_{H^{1,1}(\mathbb{R})} \leq c\|u\|_{H^{0,1}}\left\|\left(m_{2}\right)_{1}\right\|_{H^{1,1}} \leq c\|u\|_{H^{0,1}}^{2}\left\|\left(m_{2}\right)_{2}\right\|_{L^{\infty}} .
$$

As $x \rightarrow \infty$, we re-write (3.15) as

$$
\left(m_{2}\right)_{2}(x, z)=1-\int_{-\infty}^{\infty} \bar{u}(y)\left(m_{2}\right)_{1}(y, z) \mathrm{d} y+\int_{x}^{\infty} \bar{u}(y)\left(m_{2}\right)_{1}(y, z) \mathrm{d} y .
$$

The quantity $1-\int_{-\infty}^{\infty} \bar{u}(y)\left(m_{2}\right)_{1}(y, z) \mathrm{d} y$ is precisely $a(z)$. Arguing as above we see that

$$
\left\|\left(\left(m_{2}\right)_{2}-a(z)\right) \chi^{+}\right\|_{H^{1,1}(\mathbb{R})} \leq c\|u\|_{H^{0,1}}^{2}\left\|\left(m_{2}\right)_{2}\right\|_{L^{\infty}} .
$$

This completes the proof of the Proposition.

Lemma 3.6. Let $f(x) \in H^{0,1}(\mathbb{R}), g(x) \in H^{1,1}(\mathbb{R})$ and $q>0$. Then,

$$
\begin{aligned}
& \left\|\int_{\langle x\rangle}^{\infty} f(y) g(y) \mathrm{d} y\right\|_{H^{1,1}\left(\mathbb{R}^{+}\right)} \leq c\|f\|_{H^{0,1}\left(\mathbb{R}^{+}\right)}\|g\|_{H^{1,1}\left(\mathbb{R}^{+}\right)}, \\
& \left\|\int_{\langle x\rangle}^{\infty} f(y) e^{-q(y-\langle x\rangle)} \mathrm{d} y\right\|_{H^{1,1}(\mathbb{R})} \leq c\|f\|_{H^{0,1}(\mathbb{R})}, \\
& \left\|\int_{-\infty}^{\langle x\rangle} f(y) e^{-q(\langle x\rangle-y)} \mathrm{d} y\right\|_{H^{1,1}(\mathbb{R})} \leq c\|f\|_{H^{0,1}(\mathbb{R})},
\end{aligned}
$$

where $c$ depends on $q$.

Proof. For $x>0$,

$$
\begin{aligned}
\left|\int_{x}^{\infty} f(y) g(y) \mathrm{d} y\right| & \leq \int_{x}^{\infty} \frac{y^{2}+1}{x^{2}+1}|f(y)||g(y)| \mathrm{d} y \\
& \leq \frac{1}{x^{2}+1}\|f\|_{H^{0,1}\left(\mathbb{R}^{+}\right)}\|g\|_{H^{0,1}\left(\mathbb{R}^{+}\right)} .
\end{aligned}
$$

and hence we obtain the inequality for $\left\|\int_{\langle x\rangle}^{\infty} f g\right\|_{H^{0,1}\left(\mathbb{R}^{+}\right)}$. As $\frac{d}{d x} \int_{x}^{\infty} f g=$ $-f g$, the first inequality follows. For the second inequality, observe that

$$
\int_{x}^{\infty} f(y) e^{-q(y-x)} \mathrm{d} y=\int_{0}^{\infty} f(x+y) e^{-q y} \mathrm{~d} y .
$$


and hence by Minkowski's inequality,

$$
\begin{aligned}
& \left\|(1+|\langle x\rangle|) \int_{\langle x\rangle}^{\infty} f(y) e^{-q(y-\langle x\rangle)}\right\|_{L^{2}(\mathbb{R})} \\
& \leq \int_{0}^{\infty}\|(1+|\langle x\rangle|) f(\langle x\rangle+y)\|_{L^{2}(\mathbb{R})} e^{-q y} \mathrm{~d} y \\
& \leq c\|f\|_{H^{0,1}(\mathbb{R})} \int_{0}^{\infty}(1+y) e^{-q y} \mathrm{~d} y \leq c\|f\|_{H^{0,1}(\mathbb{R})} .
\end{aligned}
$$

Now, $\frac{d}{d x} \int_{x}^{\infty} f e^{q(x-y)}=-f+\int_{x}^{\infty} q f e^{q(x-y)}$, which lies in $L^{2}$, again by the Minkowski's inequality. This proves the the second inequality. A simple change of variables shows that the third inequality follows from the second inequality.

In [ZS72] the authors consider solutions $\phi$ of the Lax equation $T \phi=$ $z \phi$ which are different from, but related to, the boundary values of the Beals-Coifman solutions $\psi_{ \pm}(x, z)=\left(\psi_{1_{ \pm}}(x, z), \Psi_{2 \pm}(x, z)\right)$. For $z \in \mathbb{R}$, set

$$
\begin{aligned}
& \psi^{+}(x, z) \equiv\left(a(z) \psi_{1_{+}}(x, z), \overline{a(z)} \psi_{2_{-}}(x, z)\right), \\
& \psi^{-}(x, z) \equiv\left(\psi_{1_{-}}(x, z), \psi_{2_{+}}(x, z)\right),
\end{aligned}
$$

Then $T \psi^{ \pm}=z \psi \pm, z \in \mathbb{R}$ and $\psi^{ \pm}(x, z)$ have asymptotics,

$$
\begin{aligned}
& m^{+}(x, z) \equiv \psi^{+}(x, z) e^{-i x z \sigma} \rightarrow \mathrm{I} \text { as } x \rightarrow+\infty, \\
& m^{-}(x, z) \equiv \psi^{-}(x, z) e^{-i x z \sigma} \rightarrow \mathrm{I} \text { as } x \rightarrow-\infty,
\end{aligned}
$$

Moreover, the solutions $\psi^{ \pm}, z \in \mathbb{R}$, are uniquely determined by the conditions (3.18). From Proposition 3.1.

$$
\psi_{1}^{ \pm}(x, z)=i \sigma_{2} \overline{\psi_{2}^{ \pm}(x, \bar{z})}=\left(\begin{array}{cc}
0 & 1 \\
-1 & 0
\end{array}\right) \overline{\psi_{2}^{ \pm}(x, \bar{z})} .
$$

As det $\psi^{ \pm}(x, z) \equiv 1, z \in \mathbb{R}$, we conclude that for $x, z \in \mathbb{R}$,

$$
\left\|\psi_{j}^{ \pm}(x, z)\right\|^{2}=\left|\left(\psi_{j}^{ \pm}\right)_{1}(x, z)\right|^{2}+\left|\left(\psi_{j}^{ \pm}\right)_{2}(x, z)\right|^{2}=1, \quad j=1,2 .
$$

A direct calculation using the jump relation for $\psi_{ \pm}$shows that

$$
\psi^{+}(x, z)=\psi^{-}(x, z) S(z), \quad z \in \mathbb{R}
$$

where $S$ is the scattering matrix and

$$
S(z)=\left(\begin{array}{ll}
a(z) & -\overline{b(z)} \\
b(z) & \overline{a(z)}
\end{array}\right), \quad b(z) \equiv a(z) \overline{r(z)}, \quad z \in \mathbb{R} .
$$

We have

$$
|a(z)|^{2}+|b(z)|^{2}=\operatorname{det} S(z)=1, \quad z \in \mathbb{R} .
$$


Note that if $u$ is generic, $a(z) \neq 0$ for $z \in \mathbb{R}$ and as $a(z) \rightarrow 1$ as $|z| \rightarrow \infty,\|b\|_{L^{\infty}(\mathbb{R})} \leq c<1$. Let

$$
\Gamma_{+}=\left\{\gamma\left(z_{k}\right), z_{k} \in Z_{+}\right\}
$$

Knowledge of $\left(a, b, \Gamma_{+}\right)$is equivalent to the knowledge of $\left(r, Z_{+}, K_{+}\right)$. Indeed if $a, b$ and $\Gamma_{+}$are known, then clearly $r(z)=\overline{b(z)} / \overline{a(z)}$ and $c\left(z_{k}\right)=\frac{\gamma\left(z_{k}\right)}{a^{\prime}\left(z_{k}\right)}, k=1, \cdots, n$ are known. Moreover, $Z_{+}$is just the set of the zeros of $a(z)$ in $\mathbb{C}^{+}$. Conversely, as $|a(z)|^{-2}=1+|r(z)|^{2}$, it is easy to verify that

$$
a(z)=\prod_{z_{k} \in Z_{+}} \frac{z-z_{k}}{z-\overline{z_{k}}} e^{-l(z)} .
$$

where

$$
l(z)=\frac{1}{2 \pi i} \int_{\mathbb{R}} \frac{\log \left(1+|r(s)|^{2}\right)}{s-z} \mathrm{~d} s, \quad z \in \mathbb{C}^{+} .
$$

But then $b(z)=a(z) \overline{r(z)}, z \in \mathbb{R}$, and using the above formula (3.24) it is easy to deduce that

$$
b \in H^{1,1}(\mathbb{R}) .
$$

Also,

$$
\gamma\left(z_{k}\right)=c\left(z_{k}\right) a^{\prime}\left(z_{k}\right), \quad k=1, \cdots, n .
$$

We will use this fact repeatedly below without further comment. The solutions $\psi^{ \pm}$are called the ZS-AKNS solutions of $T \psi=z \psi$ (see [AKNS74]). (3.17) shows that $\psi_{1}^{+}$and $\psi_{2}^{-}$, the first and second columns of $\psi^{+}$and $\psi^{-}$, respectively, have analytic continuations to $\mathbb{C}^{+}$. Also, $\psi_{1}^{-}$and $\psi_{2}^{+}=\overline{a(z)} \psi_{2_{-}}(x, z)=\overline{a(\bar{z})} \psi_{2_{-}}(x, z)$ and $\psi_{2}^{+}$have analytic continuations to $\mathbb{C}^{-}$. Whenever we mention ZS-AKNS solutions in the sequel for $z \in \mathbb{C} \backslash \mathbb{R}$, we always mean the analytic continuations of $\psi^{+}$, $\psi_{2}^{-}$and $\psi_{1}^{-}, \psi_{2}^{+}$to $\mathbb{C}^{+}$(respectively $\mathbb{C}^{-}$). Note that

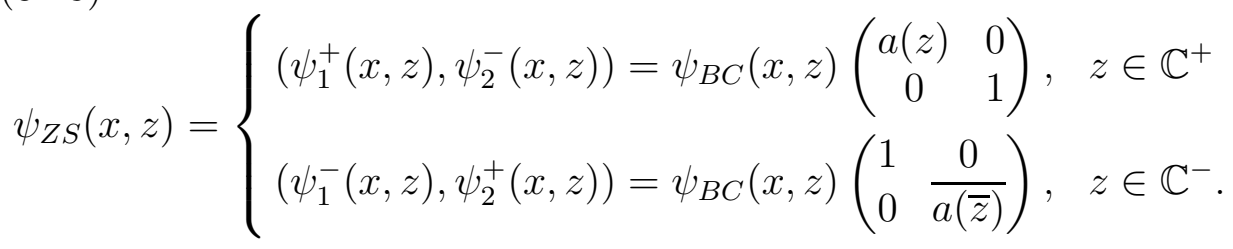

In particular, as $\operatorname{det} \psi_{B C}(x, z)=1$, we see that

$$
\operatorname{det} \psi_{Z S}(x, z)= \begin{cases}a(z), & z \in \mathbb{C}^{+} \\ \overline{a(\bar{z})}, & z \in \mathbb{C}^{-}\end{cases}
$$


Also, from (3.21), we see that

$$
b(z)=\operatorname{det}\left(\psi_{1}^{-}, \psi_{1}^{+}\right)(x, z), \quad z \in \mathbb{R} .
$$

Clearly by (3.29),$a(z)=0$ for $z \in \mathbb{C}^{+}$if and only if $\psi_{1}^{+}$is proportional to $\psi_{2}^{-}$. But as $\psi_{1}^{+}$and $\psi_{2}^{-}$decays exponentially as $x \rightarrow \pm \infty$, respectively, we see that $a(z)=0, z \in \mathbb{C}^{+}$if and only if $z$ is an $L^{2}(\mathbb{R})$-eigenvalue of the operator $T$. Similarly for $z \in \mathbb{C}^{-}, a(z)=0$ if and only if $\psi_{1}^{-}$is proportional to $\psi_{2}^{+}$. Also, $a(z)=0$ if and only if $z$ is an $L^{2}(\mathbb{R})$-eigenvalue of the operator $T$. Let $K=K_{+} \cup K_{-}=$ $\left\{c\left(z_{1}\right), \cdots, c\left(z_{n}\right)\right\} \cup\left\{c\left(\overline{z_{1}}\right), \cdots, c\left(\overline{z_{n}}\right)\right\}$.

Definition 3.7. The scattering map $\mathcal{S}$ takes $u \in \mathcal{G}$ to its scattering data $\mathcal{S}=\mathcal{S}(u)=\left(r, Z_{+}, K_{+}\right)$

$H^{1,1} \supset \mathcal{G} \ni u \mapsto \mathcal{S}(u)=\left(r(\cdot ; u), Z_{+}(u), K_{+}(u)\right) \in H^{1,1} \times \mathbb{C}_{+}^{n} \times(\mathbb{C} \backslash 0)^{n}$

where $n=n(u)$ depends on $u$.

The inverse scattering map $\mathcal{S}^{-1}$ is constructed by solving a normalized RHP (see BC84, Zho98]). The use of RHP's to solve inverse scattering problems, goes back to the work of Shabat Sha75. For any $n \geq 0$, let

- $r \in H^{1,1}$,

- $Z=Z_{+} \cup Z_{-}$where $Z_{+} \in \mathbb{C}_{+}^{n}$ and $Z_{-}=\overline{Z_{+}} \in \mathbb{C}_{-}^{n}$,

- $K=K_{+} \cup K_{-}$where $K_{+}=\left\{c\left(z_{1}\right), \cdots, c\left(z_{n}\right)\right\} \in(\mathbb{C} \backslash 0)^{n}$ and $K_{-}=\left\{c\left(\overline{z_{1}}\right)=-\overline{c\left(z_{1}\right)}, \cdots, c\left(\overline{z_{n}}\right)=-\overline{c\left(z_{n}\right)}\right\} \in(\mathbb{C} \backslash 0)^{n}$.

Set $v(z)=\left(\begin{array}{c}1+|r(z)|^{2} \\ r(z)\end{array} \quad 1\right.$ and for $x \in \mathbb{R}$,

$$
v_{x}(z)=e^{i x z \operatorname{ad} \sigma} v(z) \equiv e^{i x z \sigma} v(z) e^{-i x z \sigma}=\left(\begin{array}{cc}
1+\left|r_{x}(z)\right|^{2} & r_{x}(z) \\
r_{x}(z) & 1
\end{array}\right),
$$

where $r_{x}(z)=r(z) e^{i x z}$. For fixed $x \in \mathbb{R}$, let $m=m(x, z)$ solve the following normalized RHP:

(i) $m(x, \cdot)$ is analytic in $\mathbb{C} \backslash(\mathbb{R} \cup Z)$.

(ii) $m(x, \cdot)$ has continuous boundary values on the axis satisfying

$$
m_{+}(x, z)=m_{-}(x, z) v_{x}(z), \quad z \in \mathbb{R},
$$

(iii) $m(x, \cdot)$ has simple poles at $Z$ satisfying

$$
\begin{aligned}
& \operatorname{Res}_{z=z_{k}} m(x, z)=\lim _{z \rightarrow z_{k}} m(x, z) v_{x}\left(z_{k}\right), \quad v_{x}\left(z_{k}\right)=\left(\begin{array}{cc}
0 & 0 \\
c_{x}\left(z_{k}\right) & 0
\end{array}\right), \\
& \operatorname{Res}_{z=\bar{z}_{k}} m(x, z)=\lim _{z \rightarrow \overline{z_{k}}} m(x, z) v_{x}\left(\overline{z_{k}}\right), \quad v_{x}\left(\overline{z_{k}}\right)=\left(\begin{array}{cc}
0 & c_{x}\left(\overline{z_{k}}\right) \\
0 & 0
\end{array}\right) .
\end{aligned}
$$


where $c_{x}\left(z_{k}\right)=e^{-i x z_{k}} c\left(z_{k}\right), c_{x}\left(\overline{z_{k}}\right)=e^{i x \overline{z_{k}}} c\left(\overline{z_{k}}\right)=-\overline{c_{x}\left(z_{k}\right)}$.

(iv) $m(x, z) \rightarrow I$ as $z \rightarrow \infty$.

Note that, by Theorem 4, if $u(x, z)$ is the Beals-Coifman solution for $u(x)$, then $m(x, z) \equiv \psi(x, z) e^{-i x z \sigma}$ is a solution of the above normalized RHP with $\mathcal{S}(u)=\left(r, Z_{+}, K_{+}\right)$.

Remark 3.8. If $m=m(x, z)$ solves the above RHP, $\operatorname{det} m(x, z) \equiv 1$. Indeed, as $\operatorname{det} v_{x}(z) \equiv 1$, $\operatorname{det} m$ is analytic across $\mathbb{R}$. Also, $\operatorname{det} m$ is analytic at $z_{k}$ : this is because the residue condition is easily shown to be equivalent to the requirement that $m(x, z)=M(x, z)\left(I+\frac{v_{x}\left(z_{k}\right)}{z-z_{k}}\right)$ where $M(x, z)$ is analytic and invertible near $z=z_{k}$. On the other hand, $\operatorname{det} m(x, z) \rightarrow 1$ as $z \rightarrow \infty$ and so $\operatorname{det} m(x, z) \equiv 1$ by Liouville's theorem.

Remark 3.9. If the above RHP has a solution $m=m(x, z)$, then it is unique. Indeed, if $\hat{m}=\hat{m}(x, z)$ is another solution of the RHP, a simple standard calculation shows that the ratio $\hat{m} m^{-1}$ is an entire function which goes to I as $z \rightarrow \infty$. By Liouville's theorem, it follows that $\hat{m}=m$.

We now introduce a Cauchy operator on $\mathbb{R}$,

$$
\begin{aligned}
& C h(z)=\int_{\mathbb{R}} \frac{h(s)}{s-z} \frac{\mathrm{d} s}{2 \pi i}, \quad z \in \mathbb{C} \backslash \mathbb{R}, \\
& C^{ \pm} h(z)=\lim _{\epsilon \downarrow 0} C h(z \pm i \epsilon), \quad z \in \mathbb{R} .
\end{aligned}
$$

The operators $C^{ \pm}$are bounded, self-adjoint projections in $L^{2}(\mathbb{R}),\left\|C^{ \pm}\right\|_{L^{2}(\mathbb{R}) \rightarrow L^{2}(\mathbb{R})}=$ 1 , satisfying the important identity

$$
C^{+}-C^{-}=\mathrm{id}
$$

Define the operator on $2 \times 2$ matrix valued functions

$$
C_{v} h=C^{-}(h(v-I)), \quad h \in L^{2} .
$$

Clearly, $C_{v}$ is also bounded in $L^{2}$. For $x \in \mathbb{R}$, let $\mu=\mu_{x}(z)$ be a $2 \times 2$ matrix valued function on $\mathbb{R} \cup Z$ with the following properties. On $\mathbb{R}$, all the entries of $\mu-I=\mu(z)-I$ lie in $L^{2}(\mathbb{R})$ and for each $\zeta \in Z$, $\mu(\zeta) \in M_{2 \times 2}(\mathbb{C})$. Suppose that $\mu=\mu_{x}$ solves the following system of 
coupled singular integral equations in $\mathbb{R} \times Z$,

$$
\begin{array}{cl}
\left(\left(1-C_{v_{x}}\right) \mu_{x}\right)(z)=I+\sum_{\zeta \in Z} \frac{\mu_{x}(\zeta) v_{x}(\zeta)}{\zeta-z}, \quad z \in \mathbb{R}, & \\
\mu_{x}(\zeta)=I+\frac{1}{2 \pi i} \int_{\mathbb{R}} \frac{\mu_{x}(s)\left(v_{x}(s)-I\right)}{s-\zeta} \mathrm{d} s & \\
+\sum_{\zeta^{\prime} \in Z \backslash\{\zeta\}} \frac{\mu_{x}\left(\zeta^{\prime}\right) v_{x}\left(\zeta^{\prime}\right)}{\zeta^{\prime}-\zeta}, & \zeta \in Z .
\end{array}
$$

Set

$$
m(z)=m(x, z) \equiv I+\frac{1}{2 \pi i} \int_{\mathbb{R}} \frac{\mu_{x}(s)\left(v_{x}(s)-I\right)}{s-z} \mathrm{~d} s+\sum_{\zeta \in Z} \frac{\mu_{x}(\zeta) v_{x}(\zeta)}{\zeta-z}
$$

for $z \in \mathbb{C} \backslash(\mathbb{R} \cup Z)$. Then, a direct calculation using (3.32) shows that $m(z)$ solves the RHP (3.31). Equations (3.34) have a unique solution $\mu=\mu_{x}$ in $\left(I+L^{2}(\mathbb{R})\right) \times\left(M_{2 \times 2}(\mathbb{C})\right)^{2 n}$ by the following argument (see [Zho98] for details): First one shows that the equations are Fredholm of index zero, and then one shows that the associated kernel of the equations is $\{0\}$ (More properly, one shows that equations (3.34) are of type $\mathcal{F} \mathbf{x}=\mathbf{y}$ where $\mathcal{F}$ is a Fredholm operator with $\operatorname{ind}(\mathcal{F})=0$ and $\operatorname{Null}(\mathcal{F})=\operatorname{dim} \operatorname{Ker}(\mathcal{F})=0$. Thus $\operatorname{codim}(\mathcal{F})=-\operatorname{ind}(\mathcal{F})+\operatorname{Null}(\mathcal{F})=0$ and so $\mathcal{F}$ is a bijection). Hence the solution $\mu_{x}$ exists and is unique in $\left(I+L^{2}(\mathbb{R})\right) \times\left(M_{2 \times 2}(\mathbb{C})\right)^{2 n}$ for each $x \in \mathbb{R}$. Moreover, as $z \rightarrow \infty$ in a cone $|\operatorname{Im} z|>c|\operatorname{Re} z|, c>0$,

Set

$$
m(z)=m(x, z)=I+\frac{m_{1}(x)}{z}+o\left(\frac{1}{z}\right) .
$$

$$
u(x)=-i\left(m_{1}(x)\right)_{12} .
$$

Then one shows that $r \in H^{1,1} \Rightarrow u(x) \in H^{1,1}$.

Definition 3.10. The inverse scattering map $\mathcal{I}$ takes $\left(r, Z_{+}, K_{+}\right) \in$ $H^{1,1} \times \mathbb{C}_{+}^{n} \times(\mathbb{C} \backslash 0)^{n}$ to the function $u(x) \in H^{1,1}$,

$$
\left(r, Z_{+}, K_{+}\right) \mapsto\left(r, Z_{+} \cup \overline{Z_{+}}, K_{+} \cup\left(-\overline{K_{+}}\right)\right) \mapsto u(x) .
$$

Theorem 5. The maps $\mathcal{S}: \mathcal{G} \rightarrow H^{1,1} \times \mathbb{C}_{+}^{n} \times(\mathbb{C} \backslash 0)^{n}$ and $\mathcal{I}: H^{1,1} \times$ $\mathbb{C}_{+}^{n} \times(\mathbb{C} \backslash 0)^{n} \rightarrow \mathcal{G}$ are continuous and inverse to each other.

Remark 3.11. (see [BC84] [Zho98]; also cf. [DZ03]) The direct scattering problem yields the function $m(x, z)=m(x, z ; u)=\psi(x, z ; u) e^{-i x z \sigma}$ where $\psi$ is a Beals-Coifman solution of $\frac{d \psi}{d x}=\left(i z \sigma+\left(\begin{array}{cc}0 & u(x) \\ -u(x) & 0\end{array}\right)\right) \psi$. On the other hand, the RHP yields the function $m(x, z)=m\left(x, z ; r, Z_{+}, K_{+}\right)$. 
We will simply use the notation $m(x, z)$; the precise meaning will be clear from the context. Of course, if $\left(r, Z_{+}, K_{+}\right)=\mathcal{S}(u)$, then by the above theorem $m(x, z ; u)=m\left(x, z ; r, Z_{+}, K_{+}\right)$.

Theorem 6 (Time evolution of the scattering data: see [ZS72] [Zho89]). Suppose $u_{0} \in \mathcal{G} \subset H^{1,1}$. Then the (weak, global) solution $u(t)$ of (3.1) with $u(t=0)=u_{0}$ remains in $\mathcal{G}, u(t) \in \mathcal{G}$ for $t \geq 0$, and

$$
\begin{aligned}
& r(t)=r(u(t))=r\left(z ; u_{0}\right) e^{-i z^{2} t / 2}, \quad z \in \mathbb{R}, \\
& Z_{+}(t)=\left\{z_{j}(t)=z_{j}(u(t))=z_{j}\left(u_{0}\right), j=1, \cdots, n\right\}, \\
& K_{+}(t)=\left\{c_{j}(t)=c_{j}(u(t))=c_{j}\left(u_{0}\right) e^{i z_{j}^{2} t / 2}, j=1, \cdots, n\right\},
\end{aligned}
$$

or equivalently,

$$
\begin{aligned}
& a(t)=a(u(t))=a\left(z ; u_{0}\right), \quad z \in \overline{\mathbb{C}^{+}} \\
& b(t)=b(u(t))=b\left(z ; u_{0}\right) e^{i z^{2} t / 2}, \quad z \in \mathbb{R}, \\
& Z_{+}(t)=\left\{z_{j}(t)=z_{j}(u(t))=z_{j}\left(u_{0}\right), j=1, \cdots, n\right\} \\
& \Gamma_{+}(t)=\left\{\gamma_{j}(t)=\gamma_{j}(u(t))=\gamma_{j}\left(u_{0}\right) e^{i z_{j}^{2} t / 2}, j=1, \cdots, n\right\} .
\end{aligned}
$$

Conversely, if $r_{0} \in H^{1,1}(\mathbb{R})$ and

$$
\begin{aligned}
& Z_{+}^{0}=\left\{z_{1}^{0}, \cdots, z_{n}^{0}\right\} \subset \mathbb{C}^{+}, \\
& K_{+}^{0}=\left\{c_{1}^{0}, \cdots, c_{n}^{0}\right\} \subset \mathbb{C} \backslash\{0\},
\end{aligned}
$$

then $u(t)=\mathcal{I}\left(r_{0}(\diamond) e^{-i \diamond^{2} t / 2}, Z_{+}^{0},\left\{c_{j}^{0} e^{i\left(z_{j}^{0}\right)^{2} t / 2}\right\}\right)$ solves $N L S$ with initial data $u_{0}=\mathcal{I}\left(r_{0}, Z_{+}^{0}, K_{+}^{0}\right)$. In particular, if $u_{0} \in \mathcal{G}$ and $r_{0}=r\left(z ; u_{0}\right)$, $\left\{z_{j}^{0}=z_{j}\left(u_{0}\right)\right\},\left\{c_{j}^{0}=c_{j}\left(u_{0}\right)\right\}$, then the (weak, global) solution $u(t)$ of $N L S$ with $u(0)=u_{0}$ is given by

$$
\begin{aligned}
u(t) & =\mathcal{I}(\mathcal{S}(u(t))) \\
& =\mathcal{I}\left(r(u(t)), Z_{+}(t)=Z_{+}, K_{+}(t)\right) \\
& =\mathcal{I}\left(r\left(\diamond ; u_{0}\right) e^{-i \diamond^{2} t / 2},\left\{z_{j}\left(u_{0}\right)\right\},\left\{c_{j}\left(u_{0}\right) e^{i z_{j}^{2} t / 2}\right\}\right) .
\end{aligned}
$$

The long-time behavior of $u(t)$ is then inferred by evaluating $\mathcal{I}$ in terms of the RHP (3.31) and using the steepest descent method of DZ93.

Remark 3.12. Let $\psi_{Z S}(x, t, z) \equiv\left(\psi_{1}^{+}, \psi_{2}^{-}\right)(x, t, z)$ for $z \in \mathbb{C}^{+}$. As the operator $\partial_{t}-E$ in (3.2) commutes with $\partial_{x}-L$ in (1.28), $\left(\partial_{x}-\right.$ $L)\left(\left(\partial_{t}-E\right) \psi_{Z S}\right)=\left(\partial_{t}-E\right)\left(\left(\partial_{x}-L\right) \psi_{Z S}\right)=0$, and so $\left(\partial_{t}-E\right) \psi_{Z S}$ also solves (3.5). For $z \in \mathbb{C} \backslash \mathbb{R}$ such that $a(z)=\operatorname{det} \psi_{Z S} \neq 0$, we have $\left(\partial_{t}-E\right) \psi_{Z S}=\psi_{Z S} M$ for some matrix $M=M(z, t)$. As $\psi_{Z S} e^{-i x z \sigma}$ is 
bounded as $x \rightarrow \pm \infty$, we see that $M(z, t)=\frac{i z^{2} \sigma}{2}$, or equivalently,

$$
\frac{\partial}{\partial t}\left(\begin{array}{c}
\left(\psi_{1}^{+}\right)_{1} \\
\left(\psi_{1}^{+}\right)_{2}
\end{array}\right)=\frac{i}{2}\left(\begin{array}{cc}
|u|^{2} & u_{x}+i z u \\
\bar{u}_{x}-i z \bar{u} & z^{2}+|u|^{2}
\end{array}\right)\left(\begin{array}{c}
\left(\psi_{1}^{+}\right)_{1} \\
\left(\psi_{1}^{+}\right)_{2}
\end{array}\right)
$$

and

$$
\frac{\partial}{\partial t}\left(\begin{array}{c}
\left(\psi_{2}^{-}\right)_{1} \\
\left(\psi_{2}^{-}\right)_{2}
\end{array}\right)=\frac{i}{2}\left(\begin{array}{cc}
-z^{2}+|u|^{2} & u_{x}+i z u \\
\bar{u}_{x}-i z \bar{u} & |u|^{2}
\end{array}\right)\left(\begin{array}{c}
\left(\psi_{2}^{-}\right)_{1} \\
\left(\psi_{2}^{-}\right)_{2}
\end{array}\right)
$$

\section{The BäCKLund Transformation, the Bäcklund Extension And the Solution Procedure}

Most of the results in this section are due to Bikbaev and Tarasov [BT91] Tar91]. For completeness, what follows is a presentation of the results in BT91 Tar91 with some interpretations, additions and alternative proofs. Let $q \in \mathbb{R}$ and let $u(x)$ be an $L_{\text {loc }}^{1}$ complex-valued function on the line or the half-line $\mathbb{R}^{+}$or $\mathbb{R}^{-}$. Let $Q=\left(\begin{array}{cc}0 & u \\ -\bar{u} & 0\end{array}\right)$ as above. Consider the following $2 \times 2$ matrix ODE,

$$
\left\{\begin{array}{l}
P_{x}=(Q-i[\sigma, P]) P-P Q, \\
P_{0} \equiv P(0)=-i q \sigma_{3} .
\end{array}\right.
$$

Proposition 4.1. If $u \in L_{l o c}^{1}(\mathbb{R})$, then there exists a unique global solution $P(x)$ of (4.1) on $\mathbb{R}$. If $u \in L_{\text {loc }}^{1}\left(\mathbb{R}^{ \pm}\right)$, then there exists a unique global solution $P(x)$ of (4.1) on $\mathbb{R}^{ \pm}$, respectively.

Proof. Let $\Psi^{0}(x, z)=\left(\Psi_{1}^{0}(x, z), \Psi_{2}^{0}(x, z)\right)$ solve (3.5) with $\Psi^{0}(0, z)=I$. Equation (4.1) can be linearized in the following way. Note that (4.1) can be rewritten in an iso-spectral form

$$
P_{x}=[Q-i \sigma P, P] \text {. }
$$

Hence

$$
P(x)=\varphi(x) P_{0}(\varphi(x))^{-1} .
$$

for some invertible matrix $\varphi(x), \varphi(0)=I$. But then we must have $P_{x}=$ $\left[\varphi^{\prime}(x)(\varphi(x))^{-1}, P\right]$, so we can choose $\varphi^{\prime} \varphi^{-1}=Q-i \sigma P=Q-i \sigma \varphi P_{0} \varphi^{-1}$ or $\varphi^{\prime}=Q \varphi-i \sigma \varphi P_{0}$. Hence if $\varphi(x)=\left(\varphi_{1}(x), \varphi_{2}(x)\right)$ we see that $\varphi_{1}^{\prime}=$ $Q \varphi_{1}+i(i q) \sigma \varphi_{1}$ and $\varphi_{2}^{\prime}=Q \varphi_{2}+i(-i q) \sigma \varphi_{2}$. As $\varphi(0)=I$, we must have $\varphi(x)=\left(\Psi_{1}^{0}(x, i q), \Psi_{2}^{0}(x,-i q)\right)$. But by Proposition [3.1, $\Psi_{2}^{0}(x,-i q)=$ $\overline{\sigma_{2} \Psi^{0}(x, i q) \sigma_{2}} e_{2}=\left(\begin{array}{cc}0 & -1 \\ 1 & 0\end{array}\right) \overline{\Psi_{1}^{0}(x, i q)}$. Thus $\varphi(x)=\left(\begin{array}{cc}\xi_{1} & -\overline{\xi_{2}} \\ \xi_{2} & \overline{\xi_{1}}\end{array}\right)$, where $\xi=$ $\left(\begin{array}{l}\xi_{1} \\ \xi_{2}\end{array}\right)=\Psi_{1}^{0}(x, i q)$. In particular, $\operatorname{det} \varphi(x)=\left|\xi_{1}(x)\right|^{2}+\left|\xi_{2}(x)\right|^{2} \neq 0$ so that $\varphi(x)$ is invertible. We conclude that $P=\varphi P_{0} \varphi^{-1}, \varphi=\left(\begin{array}{ll}\xi_{1} & -\overline{\xi_{2}} \\ \xi_{2} & \overline{\xi_{1}}\end{array}\right)$ is the desired unique global solution of (4.1). 
Henceforth $P(x)$ denotes the unique solution to (4.2) for $u(x) \in L_{\text {loc }}^{1}$. For a column vector $\mathfrak{b}(x)=\left(\begin{array}{c}\mathfrak{b}_{1}(x) \\ \mathfrak{b}_{2}(x)\end{array}\right)$ such that $\mathfrak{b}(x) \neq 0$ for all $x$, define

$$
\mathcal{F}(\mathfrak{b}) \equiv \frac{\mathfrak{b}_{1} \overline{\mathfrak{b}_{2}}}{\left|\mathfrak{b}_{1}\right|^{2}+\left|\mathfrak{b}_{2}\right|^{2}}
$$

Note that $\mathcal{F}(\mathfrak{b})$ in $(4.3)$ is determined by the ratio of the entries in $\mathfrak{b}$. From (4.2) we see that

$$
P(x)=\varphi P_{0} \varphi^{-1}=\frac{-i q}{\left|\xi_{1}\right|^{2}+\left|\xi_{2}\right|^{2}}\left(\begin{array}{cc}
\left|\xi_{1}\right|^{2}-\left|\xi_{2}\right|^{2} & 2 \xi_{1} \overline{\xi_{2}} \\
2 \overline{\xi_{1}} \xi_{2} & -\left|\xi_{1}\right|^{2}+\left|\xi_{2}\right|^{2}
\end{array}\right),
$$

where $\varphi_{1}=\left(\begin{array}{l}\xi_{1} \\ \xi_{2}\end{array}\right)$. Denote

$$
\tilde{u} \equiv u-i P_{12}=u-2 q \mathcal{F}\left(\varphi_{1}\right)
$$

Then, from (4.4),

$$
\tilde{Q} \equiv Q-i[\sigma, P]=\left(\begin{array}{cc}
0 & \tilde{u} \\
-\overline{\tilde{u}} & 0
\end{array}\right)
$$

In particular, by (4.1),

$$
\tilde{u}(0)=u(0) .
$$

The choice of $P$ in (4.1) is motivated by the following fact which is easily verified by direct computation: Let $\psi(x, z)$ be a solution of (3.5),

$$
\psi_{x}=L \psi=(i z \sigma+Q) \psi
$$

and set

$$
\tilde{\psi}(x, z)=(z+P(x)) \psi(x, z) .
$$

Then $P(x)$ solves (3.5) if and only if $\tilde{\psi}(x, z)$ is an eigensolution of

$$
\tilde{\psi}_{x}=\tilde{L} \tilde{\psi}=(i z \sigma+\tilde{Q}) \tilde{\psi}, \quad \tilde{L}=i z \sigma+\tilde{Q},
$$

where $\tilde{Q}$ is given by (4.6). Equivalently, $P(x)$ solves (3.5) if and only if

$$
(z+P)\left(\partial_{x}-L\right)=\left(\partial_{x}-\tilde{L}\right)(z+P) .
$$

In particular, we see that $P(x)$ induces a Bäcklund transformation $\psi \mapsto \tilde{\psi}$ for scattering equations of type (3.5).

Remark 4.2. Note that const $=\operatorname{det} \tilde{\psi}(x, z)=\operatorname{det}(z+P(x)) \operatorname{det} \psi(x, z)=$ $\operatorname{det}(z+P(x))$ const, so $\operatorname{det}(z+P(x))$ is independent of $x$. This is consistent with the fact that (4.1) is an iso-spectral deformation $P_{0}$. 
We call the transformation $\mathcal{B}_{q}: u \mapsto \tilde{u}=\mathcal{B}_{q}(u)$, the Bäcklund transformation of $u(x)$ with respect to $q$. If $u(x)$ is only defined on $\mathbb{R}^{+}$or $\mathbb{R}^{-}$, then we call the map $\mathcal{B}_{q}^{ \pm}: u \mapsto \tilde{u}=\mathcal{B}_{q}^{ \pm}(u)$, the Bäcklund transformation of $u(x)$ with respect to $q$ on $\mathbb{R}^{+}$or $\mathbb{R}^{-}$, respectively. Clearly, $\mathcal{B}_{q}$ maps $L_{\text {loc }}^{1}(\mathbb{R}) \rightarrow L_{\text {loc }}^{1}(\mathbb{R})$ and $\mathcal{B}_{q}^{ \pm}$maps $L_{\text {loc }}^{1}\left(\mathbb{R}^{ \pm}\right) \rightarrow L_{\text {loc }}^{1}\left(\mathbb{R}^{ \pm}\right)$.

In general, Bäcklund transformations $u \mapsto \tilde{u}$ involve a choice of parameters, symbolically $\tilde{u}=F\left(u ; c_{1}, c_{2}, \cdots\right)$, for some functional $F$, which takes, for example, one scattering problem (4.8) into another (4.9), If $u(t)$ solves a dynamical equation in $t$, it is often possible to choose $c_{1}=c_{1}(t), c_{2}=c_{2}(t)$ appropriately to ensure that $\tilde{u}(t)=$ $F\left(u(t) ; c_{1}(t), c_{2}(t), \cdots\right)$ also solves an (or maybe, the same) dynamical equation. In this way the Bäcklund transformation for scattering problems, for example, gives rise to (auto-)Bäcklund transformations for the dynamical systems. For example, the Bäcklund transformation in (1.34) can also be viewed (see e.g. [Dei78]) as taking the trivial solution $W_{\text {triv }}(x, t) \equiv 0$ of $\mathrm{KdV}$ to the solution (1.34) of $\mathrm{KdV}$ via a Bäcklund transformation of Schrödinger operators

$$
H_{\text {triv }}=-\frac{d^{2}}{d x^{2}}+W_{\text {triv }} \mapsto H(t)=-\frac{d^{2}}{d x^{2}}+W(t)
$$

where $W(t)=W(x, t)=-2 \frac{d^{2}}{d x^{2}} \log \left(e^{\beta x}+c e^{-\beta x}\right)$. Only if $c$ is chosen so that $c=c(t)=q e^{8 \beta^{3} t}$, does $W_{\text {triv }} \mapsto W(t)$ result in a solution of $\mathrm{KdV}$. As we will see, the method in [BT91] Tar91 can be viewed as a Bäcklund transformation taking functions on $\mathbb{R}^{+}$to functions on $\mathbb{R}$, where the parameters are chosen automatically in such a way that the solution $u(t)=u(x, t)$ of $\mathrm{HNLS}_{q}^{+}$is taken to a solution $u^{e}(t)=u^{e}(x, t)$ of (3.1) on $\mathbb{R}$. Said differently, as $\partial_{x}-\tilde{L}$ is conjugate to $\partial_{x}-L$, $\partial_{x}-\tilde{L}=(z+P)\left(\partial_{x}-L\right)(z+P)^{-1}$, any iso-spectral deformation of $\partial_{x}-L$ will give rise to an iso-spectral deformation of $\partial_{x}-\tilde{L}$. The challenge here is to choose $P$ appropriately so that $\partial_{x}-\tilde{L}$ solves the same iso-spectral deformation (viz. NLS) as $\partial_{x}-L$.

Remark 4.3. If $q=0$, then $P(x) \equiv 0$ and $\tilde{u}(x)=u(x)$.

Let $\mathcal{R} u(x) \equiv u(-x)$. As we now show, up to the reversal $\mathcal{R}$, the Bäcklund transformation is reciprocal.

Lemma 4.4. Let $q \in \mathbb{R}$.

(i) If $u \in L_{\text {loc }}^{1}(\mathbb{R})$, then $\mathcal{R B}_{q} \mathcal{R B}_{q} u(x)=u(x), x \in \mathbb{R}$.

(ii) If $u \in L_{\text {loc }}^{1}\left(\mathbb{R}^{ \pm}\right)$, then $\mathcal{R} \mathcal{B}_{q}^{ \pm} u \in L_{\text {loc }}^{1}\left(\mathbb{R}^{\mp}\right)$ and $\mathcal{R} \mathcal{B}_{q}^{\mp} \mathcal{R} \mathcal{B}_{q}^{ \pm} u(x)=$ $u(x), x \in \mathbb{R}^{ \pm}$, respectively.

Proof. We prove (i): the proof of (ii) is similar. Suppose that $u \in$ $L_{\text {loc }}^{1}(\mathbb{R})$. Let $u_{1}=\mathcal{R B}_{q}(u)$ and let $P_{1}(x)=\sigma_{3} P(-x) \sigma_{3}$. Observe from 
(4.4) that $P(x)=-P^{*}(x)$ where $P^{*}$ is an adjoint of $P$. From (4.6),

$$
\begin{aligned}
Q(-x) & =\tilde{Q}(-x)+i[\sigma, P(-x)]=\tilde{Q}(-x)-i\left[\sigma, P_{1}(x)\right] \\
& =Q_{1}(x)-i\left[\sigma, P_{1}(x)\right]
\end{aligned}
$$

where $Q_{1}=\left(\begin{array}{cc}0 & u_{1} \\ -\bar{u}_{1} & 0\end{array}\right)$. In addition,

$$
\begin{aligned}
\left(P_{1}\right)_{x}(x) & =\left(-P_{1}^{*}\right)_{x}(x)=\sigma_{3} P_{x}^{*}(-x) \sigma_{3}=\sigma_{3}(\tilde{Q} P-P Q)^{*}(-x) \sigma_{3} \\
& =\sigma_{3}\left(-Q^{*} P^{*}+P^{*} \tilde{Q}^{*}\right)(-x) \sigma_{3}=\sigma_{3}(-Q P+P \tilde{Q})(-x) \sigma_{3} \\
& =Q(-x) P_{1}(x)-P_{1}(x) \tilde{Q}(-x)=Q(-x) P_{1}(x)-P_{1}(x) Q_{1}(x) \\
& =\left(Q_{1}(x)-i\left[\sigma, P_{1}(x)\right]\right) P_{1}(x)-P_{1}(x) Q_{1}(x) .
\end{aligned}
$$

Combining with $P_{1}(0)=P(0)$, we conclude by uniqueness that $\tilde{Q}_{1}(x)=$ $Q_{1}(x)-i\left[\sigma, P_{1}(x)\right]=Q(-x)$. Hence $\mathcal{R B}_{q}\left(u_{1}\right)=u$, as desired.

Corollary 4.5. $\mathcal{B}_{q}$ is a bijection from $L_{\text {loc }}^{1}(\mathbb{R})$ onto $L_{l o c}^{1}(\mathbb{R}) . \mathcal{B}_{q}^{ \pm}$is a bijection from $L_{\text {loc }}^{1}\left(\mathbb{R}^{ \pm}\right)$onto $L_{\text {loc }}^{1}\left(\mathbb{R}^{ \pm}\right)$, respectively.

Proof. As $\mathcal{R}^{2}=1, \mathcal{R B}_{q} \mathcal{R} \mathcal{B}_{q}=1$ implies $\mathcal{B}_{q} \mathcal{R} \mathcal{B}_{q} \mathcal{R}=1$ and the bijectivity in $L_{\text {loc }}^{1}(\mathbb{R})$ follows. Similarly, $\mathcal{R} \mathcal{B}_{q}^{\mp} \mathcal{R} \mathcal{B}_{q}^{ \pm}=1_{L_{\text {loc }}^{1}\left(\mathbb{R}^{ \pm}\right)}$and $\mathcal{B}_{q}^{ \pm} \mathcal{R} \mathcal{B}_{q}^{\mp} \mathcal{R}=$ $1_{L_{\text {loc }}^{1}\left(\mathbb{R}^{ \pm}\right)}$, which implies the bijectivity for $L_{\text {loc }}^{1}\left(\mathbb{R}^{ \pm}\right)$.

Remark 4.6. Clearly if $u \in L_{\text {loc }}^{1}(\mathbb{R})$, then

$$
\left.\left(\mathcal{B}_{q} u\right)\right|_{\mathbb{R}^{ \pm}}=\mathcal{B}_{q}^{ \pm}\left(\left.u\right|_{\mathbb{R}^{ \pm}}\right) \text {. }
$$

Proposition 4.7. $\mathcal{B}_{q}$ is a bijection from $H^{1,1}(\mathbb{R})$ onto $H^{1,1}(\mathbb{R})$. $\mathcal{B}_{q}^{ \pm}$is a bijection from $H^{1,1}\left(\mathbb{R}^{ \pm}\right)$onto $H^{1,1}\left(\mathbb{R}^{ \pm}\right)$.

Proof. We only consider the case $\mathcal{B}_{q}^{ \pm}$and $q>0$. The other cases $\mathcal{B}_{q}^{ \pm}$, $q<0$, and $\mathcal{B}_{q}, q \neq 0$ are similar. As $\mathcal{R}$ is a bijection from $H^{1,1}\left(\mathbb{R}^{ \pm}\right)$ onto $H^{1,1}\left(\mathbb{R}^{\mp}\right)$, it is enough to prove that $\mathcal{B}_{q}^{+}$and $\mathcal{B}_{q}^{-}$map $H^{1,1}\left(\mathbb{R}^{ \pm}\right)$into $H^{1,1}\left(\mathbb{R}^{ \pm}\right)$, respectively; the bijectivity of $\mathcal{B}_{q}^{+}$then follows from Lemma 4.4(ii). We consider $B_{q}^{+}$: the case $B_{q}^{-}$is similar. The proof hinges on the existence of the solutions $g(x, z)$ and $h(x, z)$ of the differential equation

$$
\psi_{x}=(i z \sigma+Q) \psi
$$

on $\mathbb{R}^{+}$with the properties that

$$
g(x, z)=\left(e_{1}+r_{1}(x, z)\right) e^{i x z / 2}, \quad r_{1} \in H^{1,1}\left(\mathbb{R}^{+}\right) .
$$

and

$$
h(x, z)=\left(e_{2}+r_{2}(x, z)\right) e^{-i x z / 2}, \quad r_{2} \in H^{1,1}\left(\mathbb{R}^{+}\right) .
$$

Note that $h$ is not unique; for any constant $c, h+c g$ solves (4.12) with (4.14) if $h$ solves (4.12) with (4.14). The solution $g=\hat{g} e^{i x z / 2}$ is 
uniquely specified by the asympotics $\hat{g}(x, z) \rightarrow e_{1}$ as $x \rightarrow \infty$, and can be constructed by solving the integral equation

$$
\hat{g}(x, z)=e_{1}-\int_{x}^{\infty}\left(\begin{array}{cc}
e^{i z(y-x) / 2} & 0 \\
0 & 1
\end{array}\right) Q(y) \hat{g}(y, z) \mathrm{d} y
$$

(cf. (3.11)) as in the proof of Proposition (3.5). The solution $h$, however, cannot be obtained in a similar way via a Volterra equation. For the reader's convenience, we prove the existence of $h$ following [CL55] pp. 104-105. Note that the non-uniqueness of $h$ is reflected in the arbitrary choice of $x_{0}$.

For any given $u \in H^{1,1}\left(\mathbb{R}^{+}\right)$, fix $x_{0}>0$ so that $\int_{x_{0}}^{\infty}|u|<\frac{1}{2}$. For $z \in$ $\mathbb{C}^{+}$, we consider the following integral equation for $g(x, z)=\left(g_{1}, g_{2}\right)^{T}$,

$$
\begin{aligned}
h(x, z)=e^{-i x z / 2} e_{2}+\int_{x_{0}}^{x}\left(\begin{array}{cc}
e^{-i z(y-x) / 2} & 0 \\
0 & 0
\end{array}\right) Q(y) h(y, z) \mathrm{d} y \\
-\int_{x}^{\infty}\left(\begin{array}{cc}
0 & 0 \\
0 & e^{i z(y-x) / 2}
\end{array}\right) Q(y) h(y, z) \mathrm{d} y, \quad x \geq x_{0} .
\end{aligned}
$$

A direct calculation shows that $h(x, z)$ solves (4.12). Setting $f(x, z)=$ $h(x, z) e^{i x z / 2}$, the integral equation (4.15) becomes

$$
f(x, z)=e_{2}+(\mathcal{T} f)(x, z), \quad x \geq x_{0},
$$

where $\mathcal{T}$ is an integral operator defined by

$$
\begin{aligned}
(\mathcal{T} f)(x, z)= & \int_{x_{0}}^{x}\left(\begin{array}{cc}
e^{-i z(y-x)} & 0 \\
0 & 0
\end{array}\right) Q(y) f(y, z) \mathrm{d} y \\
& -\int_{x}^{\infty}\left(\begin{array}{rr}
0 & 0 \\
0 & 1
\end{array}\right) Q(y) f(y, z) \mathrm{d} y, \quad f \in L^{\infty}\left[x_{0}, \infty\right) .
\end{aligned}
$$

Note that as $z \in \mathbb{C}^{+}$and $u \in L^{1}\left[x_{0}, \infty\right), \mathcal{T}$ is well-defined. Let $f_{0}=e_{2}$ and define $f_{k+1}=e_{2}+\mathcal{T} f_{k}, k \geq 0$, inductively. Then,

$$
\left\|f_{k+1}-f_{k}\right\|_{L^{\infty}\left[x_{0}, \infty\right)} \leq \frac{c}{2^{k}}, \quad k \geq 0 .
$$

Indeed, $\left\|f_{1}-f_{0}\right\|_{L^{\infty}\left[x_{0}, \infty\right)} \leq c$ and for $k \geq 1$,

$$
\begin{aligned}
& \left\|f_{k+1}-f_{k}\right\|_{L^{\infty}\left[x_{0}, \infty\right)}=\left\|\mathcal{T}\left(f_{k}-f_{k-1}\right)\right\|_{L^{\infty}\left[x_{0}, \infty\right)} \\
& \quad \leq\left\|f_{k}-f_{k-1}\right\|_{L^{\infty}\left[x_{0}, \infty\right)} \int_{x_{0}}^{\infty}|u| \leq \frac{1}{2}\left\|f_{k}-f_{k-1}\right\|_{L^{\infty}\left[x_{0}, \infty\right)} .
\end{aligned}
$$


Hence $f \equiv f_{0}+\sum_{k=1}^{\infty} f_{k}-f_{k-1}$ converges in $L^{\infty}\left[x_{0}, \infty\right)$ and solves the integral equation (4.16). Writing $f(x, z)=\left(f_{1}, f_{2}\right)^{T}$, (4.16) becomes

$$
\begin{aligned}
& f_{1}(x, z)=\int_{x_{0}}^{x} e^{-i z(y-x)} u(y) f_{2}(y, z) \mathrm{d} y \\
& f_{2}(x, z)=1+\int_{x}^{\infty} \bar{u}(y) f_{1}(y, z) \mathrm{d} y
\end{aligned}
$$

As $\|f\|_{L^{\infty}\left[x_{0}, \infty\right)} \leq c$, by Lemma [3.6, $f_{1} \in H^{1,1}\left[x_{0}, \infty\right)$ and therefore again by Lemma 3.6, $f_{2}-1 \in H^{1,1}\left[x_{0}, \infty\right)$. Now by the standard ODE theory, $h(x, z)$ extends uniquely to a solution of (4.12) on $\mathbb{R}^{+}$, also denoted by $h(x, z)$. From the above calculations, we see (4.14). Now we fix $z=i q$. As $g(x, i q)$ and $h(x, i q)$ are clearly linearly independent for $x \geq 0$,

$$
\Psi_{1}^{0}(x, i q)=c_{1} g(x, i q)+c_{2} h(x, i q) .
$$

for some constants $c_{1}, c_{2}$. By (4.4) and (4.5), we must show that $\mathcal{F}\left(\Psi_{1}^{0}(x, i q)\right) \in H^{1,1}\left(\mathbb{R}^{+}\right)$. Suppose $c_{2} \neq 0$. Then as $x \rightarrow \infty$,

$$
\Psi_{1}^{0}(x, i q)=c_{1} e^{-q x / 2}\left(\begin{array}{c}
1+r_{3}(x) \\
r_{4}(x)
\end{array}\right), \quad r_{j} \in H^{1,1}\left(\mathbb{R}^{+}\right), \quad j=3,4 .
$$

Hence

$$
\mathcal{F}\left(\Psi_{1}^{0}(x, i q)\right)=\frac{\left(1+r_{3}\right) \overline{r_{4}}}{\left|1+r_{3}\right|^{2}+\left|r_{4}\right|^{2}} \in H^{1,1}\left(\mathbb{R}^{+}\right) .
$$

On the other hand, if $c_{2} \neq 0$, then as $x \rightarrow \infty$,

$$
\Psi_{1}^{0}(x, i q)=c_{2} e^{q x / 2}\left(\begin{array}{c}
r_{5}(x) \\
1+r_{6}(x)
\end{array}\right), \quad r_{j} \in H^{1,1}\left(\mathbb{R}^{+}\right), \quad j=5,6
$$

Thus,

$$
\mathcal{F}\left(\Psi_{1}^{0}(x, i q)\right)=\frac{r_{5} \overline{\left(1+r_{6}\right)}}{\left|r_{5}\right|^{2}+\left|1+r_{6}\right|^{2}},
$$

which again lies in $H^{1,1}\left(\mathbb{R}^{+}\right)$. This completes the proof.

Lemma 4.8. Suppose $u \in H^{1,1}(\mathbb{R})$, and let $P(x)$ solve (4.1). Then, $P(x) \rightarrow-i \beta_{ \pm} \sigma_{3}$ as $x \rightarrow \pm \infty$ where $\beta_{ \pm}^{2}=q^{2}$.

Proof. From (4.4), $P(x)=\frac{-i q}{\left|\xi_{1}\right|^{2}+\left|\xi_{2}\right|^{2}}\left(\begin{array}{cc}\left|\xi_{1}\right|^{2}-\left|\xi_{2}\right|^{2} & 2 \xi_{1} \overline{\xi_{2}} \\ 2 \overline{\xi_{1}} \xi_{2} & -\left|\xi_{1}\right|^{2}+\left|\xi_{2}\right|^{2}\end{array}\right)$, where $\xi=$ $\left(\begin{array}{l}\xi_{1} \\ \xi_{2}\end{array}\right)=\Psi_{1}^{0}(x, i q), \Psi_{1}^{0}(0, i q)=e_{1}$. Suppose first that $q>0$. Then by (4.17), $\Psi_{1}^{0}(x, i q)=c_{1} g(x, i q)+c_{2} h(x, i q)$ for some constants $c_{1}$, $c_{2}$. From (4.13) (4.14), $g(x, i q)=\left(e_{1}+r_{1}(x, i q)\right) e^{-q x / 2}, h(x, i q)=$ $\left(e_{2}+r_{2}(x, i q)\right) e^{q x / 2}$ where $r_{1}(x, i q), r_{2}(x, i q) \in H^{1,1}\left(\mathbb{R}^{+}\right)$. If the second component of $g(x, i q)=\left(g_{1}(x, i q), g_{2}(x, i q)\right)^{T}$ vanishes at $x=0$, then necessarily $c_{2}=0$. But then $\frac{\xi_{2}}{\xi_{1}}=\frac{g_{2}(x, i q)}{g_{1}(x, i q)} \rightarrow 0$ as $x \rightarrow+\infty$, 
and so $P(x) \rightarrow-i q \sigma_{3}$ as $x \rightarrow+\infty$. If the second component of $g(x, i q)$ does not vanish at $x=0$, then $c_{2} \neq 0$. Then, as in (4.18), $\xi=\Psi_{1}^{0}(x, i q)=c_{2} e^{q x / 2}\left(\begin{array}{c}r_{5}(x) \\ 1+r_{6}(x)\end{array}\right)$, where $r_{5}, r_{6} \in H^{1,1}\left(\mathbb{R}^{+}\right)$. It now follows that $\frac{\xi_{1}}{\xi_{2}} \rightarrow 0$ as $x \rightarrow+\infty$ and so $P(x) \rightarrow i q \sigma_{3}$ as $x \rightarrow+\infty$. Thus $P(x) \rightarrow-i \beta_{+} \sigma_{3}$ as $x \rightarrow+\infty$, where $\beta_{+}=q$ if $g_{2}(0, i q)=0$ and $\beta_{+}=-q$ if $g_{2}(0, i q) \neq 0$.

As in the case $x \rightarrow+\infty$, there are solutions $g(x, i q), h(x, i q)$ of (4.12) with the property that $g(x, i q)=\left(e_{1}+r_{1}(x, i q)\right) e^{-q x / 2}$ and $h(x, i q)=$ $\left(e_{2}+r_{2}(x, i q)\right) e^{q x / 2}$ where $r_{1}(x, i q)$ and $r_{2}(x, i q)$ now belong to $H^{1,1}\left(\mathbb{R}^{-}\right)$. (As opposed to the case $x>0, h(x, i q)$ is now uniquely determined and $g(x, i q)$ is not unique). Again $\xi=\Psi_{1}^{0}(x, i q)=c_{1} g(x, i q)+c_{2} h(x, i q)$. If the second component of $h(x, i q)$ vanishes at $x=0$, then $c_{1}=0$. Hence $\frac{\xi_{1}}{\xi_{2}}=\frac{h_{1}(x, i q)}{h_{2}(x, i q)} \rightarrow 0$ as $x \rightarrow-\infty$, and so $P(x) \rightarrow-i q \sigma_{3}$ as $x \rightarrow-\infty$. On the other hand, as before, if the second component of $h(x, i q)$ does not vanish at $x=0$, then $c_{1} \neq 0$ and we find that $P(x) \rightarrow i q \sigma_{3}$ as $x \rightarrow-\infty$. Thus $P(x) \rightarrow-i \beta_{-} \sigma_{3}$ as $x \rightarrow-\infty$, where $\beta_{-}=q$ if $h_{2}(0, i q)=0$ and $\beta_{-}=-q$ if $h_{2}(0, i q) \neq 0$. If $q<0$, the situation is similar, and again $\beta_{ \pm}^{2}=q^{2}$.

Remark 4.9. From the above proof, we see that as $x \rightarrow \infty$, if $\Psi_{1}^{0}(x, i q)$ grows exponentially, then $\beta_{+}=-|q|$ and if $\Psi_{1}^{0}(x, i q)$ decays exponentially, then $\beta_{+}=|q|$. On the other hand, as $x \rightarrow-\infty$, if $\Psi_{1}^{0}(x, i q)$ grows exponentially, then $\beta_{-}=|q|$ and if $\Psi_{1}^{0}(x, i q)$ decays exponentially, then $\beta_{-}=-|q|$. For example, if $q>0$ and $a(i|q|) \neq 0$, then $\Psi_{1}^{0}(x, i q)=c_{1} \psi_{1}^{+}(x, i q)+c_{2} \psi_{2}^{-}(x, i q)$ for some constants $c_{1}, c_{2}$. Hence,

$$
\begin{aligned}
&\left(\psi_{1}^{+}\right)_{2}(0, i q)=0 \Longleftrightarrow c_{2}=0 \Longleftrightarrow \beta_{+}=\beta_{-}=q, \\
&\left(\psi_{2}^{-}\right)_{2}(0, i q)=0 \Longleftrightarrow c_{1}=0 \Longleftrightarrow \beta_{+}=\beta_{-}=-q, \text { and } \\
&\left(\psi_{1}^{+}\right)_{2}(0, i q) \neq 0,\left(\psi_{2}^{-}\right)_{2}(0, i q) \neq 0 \Longleftrightarrow c_{1} \neq 0, c_{2} \neq 0 \\
& \Longleftrightarrow \beta_{+}=-q, \quad \beta_{-}=q .
\end{aligned}
$$

The following calculation is standard in the inverse scattering literature.

Proposition 4.10. Let $q \in \mathbb{R} \backslash\{0\}$ be given. Suppose $u \in H^{1,1}(\mathbb{R})$ and let $\tilde{u}=\mathcal{B}_{q} u \in H^{1,1}(\mathbb{R})$. Let $S(z)=\left(\begin{array}{cc}a(z) & -\overline{\overline{b(z)}} \\ b(z) & \overline{a(z)}\end{array}\right), \tilde{S}(z)=\left(\begin{array}{ll}\tilde{a}(z) & -\overline{\tilde{b}(z)} \\ \tilde{b}(z) & \overline{\tilde{a}(z)}\end{array}\right)$ be the scattering matrices for $u$ and $\tilde{u}$, respectively. Then,

$$
\tilde{S}(z)=\left(z-i \beta_{-} \sigma_{3}\right) S(z)\left(z-i \beta_{+} \sigma_{3}\right)^{-1}, \quad z \in \mathbb{R},
$$

where $P(x) \rightarrow-i \beta_{ \pm} \sigma_{3}$ as $x \rightarrow \pm \infty, \beta_{ \pm}^{2}=q^{2}$. Equivalently,

$$
\tilde{a}(z)=\frac{z-i \beta_{-}}{z-i \beta_{+}} a(z), \quad \tilde{b}(z)=\frac{z+i \beta_{-}}{z-i \beta_{+}} b(z), \quad z \in \mathbb{R} .
$$


In particular, if $a(i|q|) \neq 0$, then

$$
\tilde{u} \in \mathcal{G} \text {, if } u \in \mathcal{G} \text {. }
$$

Moreover,

$$
\tilde{Z}_{+}=Z_{+} \text {or } \tilde{Z}_{+}=Z_{+} \cup\{i|q|\}
$$

In both cases,

$$
\tilde{\gamma}\left(z_{k}\right)=\frac{z_{k}+i \beta_{-}}{z_{k}-i \beta_{+}} \gamma\left(z_{k}\right), \quad z_{k} \in Z_{+}
$$

and in the second case,

$$
\tilde{\gamma}(i|q|)= \begin{cases}\frac{\left(\psi_{1}^{+}\right)_{2}(0, i q)}{\left(\psi_{2}^{-}\right)_{2}(0, i q)}, & \text { if } q>0, \\ \frac{\left(\psi_{1}^{+}\right)_{1}(0,-i q)}{\left(\psi_{2}^{-}\right)_{1}(0,-i q)}, & \text { if } q<0 .\end{cases}
$$

Proof. From (4.9) and Lemma 4.8, we see that

$$
\tilde{\psi}^{ \pm}(x, z)=(z+P(x)) \psi^{ \pm}(x, z)\left(z-i \beta_{ \pm} \sigma_{3}\right)^{-1}, \quad z \in \mathbb{R},
$$

where $\psi^{ \pm}, \tilde{\psi}^{ \pm}$are the ZS-AKNS solutions for $u$ and $\tilde{u}$, respectively. Substituting these relations into $\tilde{\psi}^{+}=\tilde{\psi}^{-} \tilde{S}$ and utilizing $\psi^{+}=\psi^{-} S$, we immediately obtain (4.20) and hence (4.21).

Now suppose that $u \in \mathcal{G}$ and $a(i|q|) \neq 0$. Then $\tilde{a}(z) \neq 0, z \in \mathbb{R}$, and $\tilde{a}(z) \rightarrow 1$ as $z \rightarrow \infty$. Then relationship $\tilde{a}(z)=\frac{z-i \beta_{-}}{z-i \beta_{+}} a(z)$ continues analytically to $\mathbb{C}^{+} \backslash\left\{i \beta_{+}\right\}$. Now clearly $i \beta_{ \pm} \notin Z_{+}$. Hence $\tilde{a}\left(z_{k}\right)=0$ if and only if $a\left(z_{k}\right)=0$. and as $a^{\prime}\left(z_{k}\right) \neq 0$, it follows that $z_{k}$ is a simple zero for $\tilde{a}(z)$. From (4.26), we have by analytic continuations

$$
\tilde{\psi}_{1}^{+}\left(x, z_{k}\right)=\frac{z_{k}+P(x)}{z_{k}-i \beta_{+}} \psi_{1}^{+}\left(x, z_{k}\right), \quad \tilde{\psi}_{2}^{-}\left(x, z_{k}\right)=\frac{z_{k}+P(x)}{z_{k}+i \beta_{-}} \psi_{2}^{-}\left(x, z_{k}\right) .
$$

Substituting these relations into $\tilde{\psi}_{1}^{+}\left(x, z_{k}\right)=\tilde{\gamma}\left(z_{k}\right) \tilde{\psi}_{2}^{-}\left(x, z_{k}\right)$ and utilizing $\psi_{1}^{+}\left(x, z_{k}\right)=\gamma\left(z_{k}\right) \psi_{2}^{-}\left(x, z_{k}\right)$, we immediately obtain (4.24). From (4.21) we see that $a(i|q|)=0$ if and only if $\beta_{+}=-|q|, \beta_{-}=|q|$. In this case, the zero at $i|q|$ is also simple. In particular, $\tilde{u} \in \mathcal{G}$ in both cases in (4.23). From (4.26), for $z=i|q|, \tilde{f}^{+}(x) \equiv \frac{i|q|+P(x)}{2 i|q|} \psi_{1}^{+}(x, i|q|)$ and $\tilde{f}^{-}(x) \equiv \frac{i|q|+P(x)}{2 i|q|} \psi_{2}^{-}(x, i|q|)$ are two solutions of $\frac{d \tilde{\psi}}{d x}=(-|q| \sigma+\tilde{Q}) \tilde{\psi}$ with asymptotics, $\frac{1}{2 i|q|}\left(\begin{array}{cc}i\left(|q|-\beta_{+}\right) & 0 \\ 0 & i\left(|q|+\beta_{+}\right)\end{array}\right)\left(e_{1}+o(1)\right) e^{-|q| x / 2}$ and $\frac{1}{2 i|q|}\left(\begin{array}{cc}i\left(|q|-\beta_{-}\right) & 0 \\ 0 & i\left(|q|+\beta_{-}\right)\end{array}\right)\left(e_{2}+\right.$ $o(1)) e^{|q| x / 2}$ as $x \rightarrow \pm \infty$, respectively. Hence $\tilde{f}^{+}(x)=\tilde{\psi}_{1}^{+}(x, i|q|)$, 
$\tilde{f}^{-}(x)=\tilde{\psi}_{2}^{-}(x, i|q|)$. By definition $\tilde{f}^{+}(x)=\tilde{\gamma}(i|q|) \tilde{f}^{-}(x)$. Evaluating this relation at $x=0$, we find

$$
\left(\begin{array}{cc}
i(|q|-q) & 0 \\
0 & i(|q|+q)
\end{array}\right) \psi_{1}^{+}(0, i|q|)=\tilde{\gamma}(i|q|)\left(\begin{array}{cc}
i(|q|-q) & 0 \\
0 & i(|q|+q)
\end{array}\right) \psi_{2}^{-}(0, i|q|)
$$

and so (4.25) follows. It is easy to see that $\tilde{\gamma}(i|q|)$ is non-zero and finite.

In general, if $u(t)$ solves NLS, $\tilde{u}(t)=\mathcal{B}_{q} u(t)$ will not solve NLS, because $\beta_{ \pm}(t)$ are not continuous functions of $t$. For example, we see from the proof of Lemma 4.8 that for $q>0$, say, $\beta_{+}=q$ if $g_{2}(0, i q)=0$ and $\beta_{+}=-q$ if $g_{2}(0, i q) \neq 0$. But $g=\left(g_{1}, g_{2}\right)^{T}$ is just the ZS-AKNS solution $\psi_{1}^{+}(x, i q)=\psi_{1}^{+}(x, i q ; u)$ and there is no reason why $\left(\psi_{1}^{+}\right)_{2}(0, i q)$ cannot pass through 0 as $u(t)$ evolves under NLS. In particular, we may have $\left(\psi_{1}^{+}\right)_{2}(0, i q ; u(t=0))=0$ but $\left(\psi_{1}^{+}\right)_{2}(0, i q ; u(t)) \neq 0$ for $t>0$ and hence $\beta_{+}(t)$ is not continuous at $t=0$.

Now from (4.19) $\tilde{u}$ has an eigenvalue at $z=i q, q>0$, if and only if $\left(\psi_{1}^{+}\right)_{2}(0, i q ; u)$ and $\left(\psi_{2}^{-}\right)_{2}(0, i q ; u)$ are both non-zero. Thus if $\left(\psi_{1}^{+}\right)_{2}(0, i q ; u(t))=0$ for $t=0$ but $\left(\psi_{1}^{+}\right)_{2}(0, i q ; u(t))$ and $\left(\psi_{2}^{-}\right)_{2}(0, i q ; u(t))$ are non-zero for $t>0$, we see by (4.21) that $\partial_{x} \tilde{\psi}=(i z \sigma+\tilde{Q}) \tilde{\psi}$ has no $L^{2}$-eigenvalue at $z=i q$ for $t=0$, but $\partial_{x} \tilde{\psi}=(i z \sigma+\tilde{Q}) \tilde{\psi}$ has an $L^{2}$-eigenvalue at $z=i q$ for $t>0$. Furthermore, in the case when $\left(\psi_{1}^{+}\right)_{2}(0, i q ; u(t))$ and $\left(\psi_{2}^{-}\right)_{2}(0, i q ; u(t))$ are both non-zero, the norming constant $\tilde{\gamma}(i|q|)$ in (4.25) will not, in general, evolve appropriately, i.e. $\tilde{\gamma}(i|q| ; u(t)) \neq \tilde{\gamma}(i|q| ; u(0)) e^{-i q^{2} t / 2}$. In terms of the discussion preceding Remark 4.3, the "parameters" in the Bäcklund transformation $u \mapsto \tilde{u}=\mathcal{B}_{q} u$ are not chosen correctly.

As we now show, however, if $u(t)$ solves NLS on $\mathbb{R}^{+}$together with the boundary condition (1.2), then $\tilde{u}(t)=\mathcal{B}_{q}^{+} u(t)$ is also a solution of NLS on $\mathbb{R}^{+}$(but with $q$ replaced by $-q$ ). Thus in this case, the "parameters" in the Bäcklund transformation are correctly chosen automatically. The following lemma gives the necessary and sufficient conditions on $P=P(x, t)$ in order that $\mathcal{B}_{q}^{+} u(t)$ solves NLS.

Lemma 4.11. Let $u(t)$ be a classical solution in $H^{1,1}$. Suppose that $P$ solves (4.1) and let $\tilde{u}(t)=\mathcal{B}_{q}^{+} u(t)$ be the Bäcklund transformation of $u(t)$. Let $L, \tilde{L}$ denote the Lax operators in (1.28) and define $E, \tilde{E}$ as in (3.2) with $u, \tilde{u}$, respectively. Then,

$$
\left(\partial_{t}-\tilde{E}\right)(z+P)=(z+P)\left(\partial_{t}-E\right)
$$

if and only if

$$
\left(\partial_{t}-\tilde{E}\right)\left(\partial_{x}-\tilde{L}\right)=\left(\partial_{x}-\tilde{L}\right)\left(\partial_{t}-\tilde{E}\right)
$$


In other words, condition (4.27) is necessary and sufficient for $\tilde{u}(t)$ to solve NLS.

Proof. If (4.27) holds, then by (3.3) and (4.10),

$$
\begin{aligned}
\left(\partial_{t}\right. & -\tilde{E})\left(\partial_{x}-\tilde{L}\right)(z+P) \\
& =\left(\partial_{t}-\tilde{E}\right)(z+P)\left(\partial_{x}-L\right)=(z+P)\left(\partial_{t}-E\right)\left(\partial_{x}-L\right) \\
& =(z+P)\left(\partial_{x}-L\right)\left(\partial_{t}-E\right)=\left(\partial_{x}-\tilde{L}\right)(z+P)\left(\partial_{t}-E\right) \\
& =\left(\partial_{x}-\tilde{L}\right)\left(\partial_{t}-\tilde{E}\right)(z+P),
\end{aligned}
$$

which clearly proves (4.28). Conversely, suppose that (4.28) holds. Define $Q_{1} \equiv\left(\begin{array}{cc}i|u|^{2} & i u_{x} \\ i \bar{u}_{x} & -i|u|^{2}\end{array}\right)$ and similarly $\tilde{Q}_{1}$ with $u$ replaced by $\tilde{u}$. Then,

$$
\tilde{Q}_{1}-Q_{1}=P_{x}=(\tilde{Q} P-P Q) .
$$

Indeed, $(\tilde{Q} P-P Q)_{12}=\left(P_{12}\right)_{x}=i(\tilde{u}-u)_{x}=\left(\tilde{Q}_{1}-Q_{1}\right)_{12}$ and $(\tilde{Q} P-$ $P Q)_{11}=\tilde{u} P_{21}-(-\bar{u}) P_{12}=i\left(|\tilde{u}|^{2}-|u|^{2}\right)=\left(\tilde{Q}_{1}-Q_{1}\right)_{11}$. Equation (4.29) now follows by symmetry. Set

$$
\Delta \equiv\left(\partial_{t}-\tilde{E}\right)(z+P)-(z+P)\left(\partial_{t}-E\right) .
$$

Then, by (4.6) and (4.29),

$$
\begin{aligned}
\Delta= & P_{t}-z(\tilde{E}-E)-(\tilde{E} P-P E) \\
= & \frac{1}{2} z^{2}(\tilde{Q}-Q+i[\sigma, P])-\frac{z}{2}\left(\tilde{Q}_{1}-Q_{1}-(\tilde{Q} P-P Q)\right) \\
& \quad+\left(P_{t}-\frac{1}{2}\left(\tilde{Q}_{1} P-P Q_{1}\right)\right) \\
& =P_{t}-\frac{1}{2}\left(\tilde{Q}_{1} P-P Q_{1}\right) .
\end{aligned}
$$

In particular, $\Delta$ is independent of $z$. Again by (3.3) and (4.10), we see that

$$
\begin{aligned}
\left(\partial_{x}\right. & -\tilde{L})\left(\partial_{t}-\tilde{E}\right)(z+P) \\
& =\left(\partial_{t}-\tilde{E}\right)\left(\partial_{x}-\tilde{L}\right)(z+P)=\left(\partial_{t}-\tilde{E}\right)(z+P)\left(\partial_{x}-L\right) \\
& =\Delta\left(\partial_{x}-L\right)+(z+P)\left(\partial_{t}-E\right)\left(\partial_{x}-L\right) \\
& =\Delta\left(\partial_{x}-L\right)+(z+P)\left(\partial_{x}-L\right)\left(\partial_{t}-E\right) \\
& =\Delta\left(\partial_{x}-L\right)+\left(\partial_{x}-\tilde{L}\right)(z+P)\left(\partial_{t}-E\right) \\
& =\Delta\left(\partial_{x}-L\right)-\left(\partial_{x}-\tilde{L}\right) \Delta+\left(\partial_{x}-\tilde{L}\right)\left(\partial_{t}-\tilde{E}\right)(z+P)
\end{aligned}
$$


and hence

$$
\begin{aligned}
& \Delta\left(\partial_{x}-L\right)-\left(\partial_{x}-\tilde{L}\right) \Delta=0, \quad z \in \mathbb{C} \\
& \Longleftrightarrow i z[\sigma, \Delta]-\left(\Delta_{x}-\tilde{Q} \Delta+\Delta Q\right)=0, \quad z \in \mathbb{C} \\
& \Longleftrightarrow[\sigma, \Delta]=0, \quad \Delta_{x}=\tilde{Q} \Delta-\Delta Q .
\end{aligned}
$$

The first equation above implies that $\Delta$ is diagonal. But then, $\tilde{Q} \Delta$ and $\Delta Q$ are off-diagonal and it follows that $\Delta_{x}=0$, which implies that $\Delta$ is constant in $x$. As $\tilde{u}(0)=u(0)$ and $P(x=0, t)=-i q \sigma_{3},\left(\tilde{Q}_{1} P-P Q_{1}\right)$ is off-diagonal at $x=0$ and $P_{t}(x=0)=0$, and it follows from (4.30) that $\Delta$ is also off-diagonal at $x=0$, and hence we must have $\Delta=0$ at $x=0$. Thus, $\Delta \equiv 0$ for all $x$, which completes the proof.

In motivating the approach in [BT91] and [Tar91], the following result plays a key role.

Proposition 4.12. Let $q \in \mathbb{R} \backslash\{0\}$ be given. Let $t \mapsto u(t)=u(x, t), x \geq$ 0 be $C^{2}$ with respect to $x$ and $C^{1}$ with respect to $t$ such that $u_{x x}$ and $u_{t}$ decay rapidly as $x \rightarrow+\infty$. Let $u_{0}(x)=u(x, 0)$. Then, the following statements are equivalent.

(i) $u(t)$ solves $H N L S_{q}^{+}$with initial data $u_{0}$.

(ii) $\mathcal{B}_{q}^{+} u(t)$ solves $H N L S_{-q}^{+}$with initial data $\mathcal{B}_{q}^{+} u_{0}$.

(iii) $\mathcal{R B}_{q}^{+} u(t)$ solves $H N L S_{q}^{-}$with initial data $\mathcal{R B}_{q}^{+} u_{0}$.

Proof. Clearly (ii) and (iii) are equivalent. We will first show that (i) implies (ii). Suppose that $q>0$. The case $q<0$ is similar and will be discussed below. Define $E$ as in $(\underline{3.2})$ and $\tilde{E}$ with $u$ replaced by $\tilde{u}=\mathcal{B}_{q}^{+} u$.

Claim: (i) implies that $\left(\partial_{t}-\tilde{E}\right)(z+P)=(z+P)\left(\partial_{t}-E\right), x, t \geq 0$.

Let $g(x, t, z)=\left(g_{1}, g_{2}\right)^{T}, x \geq 0, z \in \overline{\mathbb{C}^{+}}$be as in the proof of Proposition 4.7 with $u(x)=u(x, t)$. As the operator $\partial_{t}-E$ in (3.2) commutes with $\partial_{x}-L$ in (1.28), $\left(\partial_{x}-L\right)\left(\left(\partial_{t}-E\right) g\right)=\left(\partial_{t}-E\right)\left(\left(\partial_{x}-L\right) g\right)=0$, and so $\left(\partial_{t}-E\right) g$ also solves (3.5). As $\left(\partial_{t}-E\right) g e^{-i x z / 2} \rightarrow \frac{i z^{2}}{4} e_{1}$ as $x \rightarrow \infty$, we see that $\left(\partial_{t}-E\right) g=\frac{i z^{2}}{4} g$, or equivalently,

$$
\frac{\partial g}{\partial t}=\frac{i}{2}\left(\begin{array}{cc}
|u|^{2} & u_{x}+i z u \\
\bar{u}_{x}-i z \bar{u} & z^{2}+|u|^{2}
\end{array}\right) g .
$$

If $g_{2}(0, i q, t=0)=0$, then as $u_{x}(0, t)+q u(0, t)=0$, it follows from (4.31) that $g_{2}(0, i q, t)=0$ for all $t \geq 0$. Let $P(x, t)$ solve (4.1) with $u(x, t)$ and $P(x, t) \rightarrow-i \beta_{+}(t) \sigma_{3}$ as $x \rightarrow \infty$. From (4.17), we see that $\Psi_{1}^{0}(x, i q, t)=c_{1}(t) g(x, i q, t), c_{1}(t) \neq 0$ and hence $\beta_{+}(t)=q$ by Remark 4.9. From (4.2), we have $P(x, t)=\varphi(x, t) P_{0}(\varphi(x, t))^{-1}$ where 
$\varphi=\left(\varphi_{1}, \varphi_{2}\right)=\left(\Psi_{1}^{0}(x, i q, t), \Psi_{2}^{0}(x,-i q, t)\right)$. Now, $\left(\varphi_{1}\right)_{t}=\left(c_{1} g(i q)\right)_{t}=$ $\left(c_{1}^{\prime} / c_{1}-i q^{2} / 4+E(i q)\right) \varphi_{1}$. As $\varphi_{2}=\left(\begin{array}{cc}0 & -1 \\ 1 & 0\end{array}\right) \overline{\varphi_{1}}$, we have $\left(\varphi_{2}\right)_{t}=\overline{\left(c_{1}^{\prime} / c_{1}\right)}+$ $\left.i q^{2} / 4+E(-i q)\right) \varphi_{2}$ and hence $\varphi_{t}=\varphi D+\frac{1}{2}\left(i q^{2} \sigma \varphi-i q Q \varphi \sigma_{3}+Q_{1} \varphi\right)$,

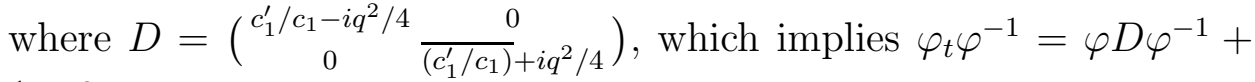
$\frac{1}{2}\left(i q^{2} \sigma+Q P+Q_{1}\right)$, Thus, from the above calculations together with $P^{2}=-q^{2} I$,

$$
\begin{aligned}
P_{t} & =\left(\varphi_{t} \varphi^{-1}\right) P-P\left(\varphi_{t} \varphi^{-1}\right) \\
& =\frac{1}{2}\left(i q^{2}[\sigma, P]+(Q P-P Q) P+Q_{1} P-P Q_{1}\right) \\
& =\frac{1}{2}\left((\tilde{Q}-Q) P^{2}+(Q P-P Q) P+Q_{1} P-P Q_{1}\right) \\
& =\frac{1}{2}\left(\tilde{Q}_{1} P-P Q_{1}\right),
\end{aligned}
$$

and hence the Claim follows from (4.30). But then, from Lemma 4.11 we see that the operators $\partial_{t}-\tilde{E}, \partial_{x}-\tilde{L}$ commute with each other. Hence, $\tilde{u}(t)$ solves NLS on $\mathbb{R}^{+}$. As $\tilde{Q}(0)=Q(0), \tilde{Q}_{x}(0)=Q_{x}(0)-$ $i\left[\sigma, P_{x}(0)\right]=-q Q(0)-i\left[\sigma, \tilde{Q}(0) P_{0}-P_{0} Q(0)\right]=q \tilde{Q}(0)$ and hence $\tilde{u}(t)$ solves $\mathrm{HNLS}_{-q}^{+}$.

If $g_{2}(0, i q, t=0) \neq 0$, then from Remark 4.9, we have $\beta_{+}(t=0)=$ $-q$. Set $v_{0}=\mathcal{R B}_{q}^{+} u_{0} \in H^{1,1}\left(\mathbb{R}^{-}\right)$and let $v(t)$ solve $\mathrm{HNLS}_{q}^{-}$with initial data $v_{0}$. Let $G(x, t, z)=\left(G_{1}, G_{2}\right)^{T}$ be the (unique) solution of the spectral problem $\left(\partial_{x}-\left(i z \sigma+\left(\begin{array}{cc}0 & v(t) \\ -\overline{v(t)} & 0\end{array}\right)\right) G=0\right.$ such that $G(x, t, z) e^{i x z / 2} \rightarrow e_{2}$ as $x \rightarrow-\infty, z \in \overline{\mathbb{C}^{+}}$. Let $P(x, t)$ be as above. Then by the proof of (4.36),

$$
G(x, t, z=0)=\frac{1}{z+i \beta_{+}(t=0)} \sigma_{1}(z-\overline{P(-x, t=0)}) \overline{g(-x,-\bar{z}, t=0)} .
$$

In particular, we see that $G_{2}(0, i q, t=0)=0$ and it follows that $\Psi_{1}^{0}\left(x, i q ; v_{0}\right)=c_{1} G(x, i q, t=0), x \leq 0$. We are now in a similar situation to the case $g_{2}(0, i q, t=0)=0$, but for $x \leq 0$, and we can conclude that $\mathcal{R B}_{q}^{-} v(t)$ solves $\mathrm{HNLS}_{q}^{+}$with initial data $\mathcal{R} \mathcal{B}_{q}^{-} \mathcal{R B}_{q}^{+} u_{0}=$ $u_{0}$. Hence $u(t)=\mathcal{R B}_{q}^{-} v(t)$, which implies that $\mathcal{R B}_{q}^{+} u(t)(=v(t))$ solves $\operatorname{HNLS}_{q}^{-}$. This shows that (i) $\Longrightarrow\left(\right.$ iii) in the case $g_{2}(0, i q, t=0) \neq 0$, and so completes the proof of the implication (i) $\Longrightarrow$ (ii), (iii).

Conversely, if (ii) holds, then $\mathcal{R B}_{q}^{+} u(t)$ solves $\operatorname{HNLS}_{q}^{-}$with initial data $\mathcal{R B}_{q}^{+} u_{0}$. As we showed above, $u(t)=\mathcal{R B}_{q}^{-}\left(\mathcal{R B}_{q}^{+} u(t)\right)$ solves $\operatorname{HNLS}_{q}^{+}$with initial data $\mathcal{R B}_{q}^{-}\left(\mathcal{R B}_{q}^{+} u_{0}\right)=u_{0}$, which proves (i). 
If $q<0$, we consider the solution $g=g(x, z)$ of $\left(\partial_{x}-L\right) g=0, x \geq 0$, $z \in \overline{\mathbb{C}^{-}}$, with $g(x, z) e^{i x z / 2} \rightarrow e_{2}$ as $x \rightarrow+\infty$. Then, mutatis mutandis, the proof goes through as above.

We will use the following definition.

Definition 4.13. Let $u \in L_{\text {loc }}^{1}\left(\mathbb{R}^{+}\right)$. Let $q \in \mathbb{R}$ and define

$$
u^{e}(x) \equiv\left\{\begin{array}{l}
u(x), x \geq 0, \\
\mathcal{R B}_{q}^{+} u(x), x<0 .
\end{array}\right.
$$

Then, $u^{e}$ is called the Bäcklund extension of $u \in L_{\text {loc }}^{1}\left(\mathbb{R}^{+}\right)$to $\mathbb{R}$ with respect to $q$.

Remark 4.14. We can also define the Bäcklund extension of $u \in L_{\text {loc }}^{1}\left(\mathbb{R}^{-}\right)$:

$$
u^{e}(x) \equiv\left\{\begin{array}{l}
\mathcal{R B}_{q}^{-} u(x), x \geq 0 \\
u(x), x<0
\end{array}\right.
$$

The following procedure, which is motivated by Proposition 4.12, shows how to express the solution of the IVP (1.4) with even initial data $u_{0} \in H^{1,1}(\mathbb{R})$ in terms of a solution of NLS (3.1). In the generic case, the latter problem can be evaluated asymptotically as $t \rightarrow \infty$ using RHP/steepest descent methods. In this way we are able to infer the long-time behavior of solutions of (1.4).

Theorem 7 (Solution procedure for the IVP (1.4) with even initial data; [BT91]). Let $q \in \mathbb{R} \backslash\{0\}$ be given and let $u_{0}$ be an even function in $H^{1,1}(\mathbb{R})$.

Step1. Set $u_{0}^{+}(x)=u_{0}(x), x \geq 0$. Let $u_{0}^{e}$ be the Bäcklund extension of $u_{0}^{+}$.

Step2. Let $u^{e}(x, t)$ be the (unique, weak global) solution of $N L S$ (3.1) in $H^{1,1}(\mathbb{R})$ with initial data $u^{e}(x, t=0)=u_{0}^{e}(x)$, in the sense of (1.10).

Step3. Set $u(x, t) \equiv u^{e}(|x|, t)$.

Then $\left.u^{e}\right|_{\mathbb{R}^{+}}(x, t), u(x, t)$ are the (unique, weak global) solutions of $H N L S_{q}^{+}$, (1.4) in the sense of (1.9), (1.8), respectively.

Up till this point, what we have shown is that if we have a solution $u^{+}(x, t)$ of $\mathrm{HNLS}_{q}^{+}$with certain technical properties, then $u^{+}(x, t)$ should be constructible via the solution procedure in Theorem[7. What we have to show, is that the solution procedure in Theorem 7 indeed produce the solution $u^{+}(x, t)$ to $\operatorname{HNLS}_{q}^{+}$with $u^{+}(x, t=0)=u_{0}^{+}(x) \in$ 
$H^{1,1}\left(\mathbb{R}^{+}\right)$. The proof of this fact involves various technicalities, and will be given in the Proof of Theorem 7 below at the end of this section.

By Theorem 7, if $u^{+}(x, t)$ solves $\operatorname{HNLS}_{q}^{+}$, then $u^{e}(x, t)$ is a solution of NLS on $\mathbb{R}$ with the property that $\mathcal{B}_{q} u^{e}(t)$ is also a solution of NLS. The following proposition provides a converse to this statement.

Proposition 4.15. Let $u(t)$ be a classical solution of $N L S$ (3.1) on $\mathbb{R}$ with $u(t=0)=u_{0} \in H^{1,1}(\mathbb{R})$. Suppose that $a(i|q|) \neq 0$ where $a(z)$ is the scattering function for $u_{0}$. If $\mathcal{B}_{q} u(t)$ solves $N L S$ on $\mathbb{R}$, then $\left.u(t)\right|_{\mathbb{R}^{+}}$ solves $H N L S_{q}^{+}$.

Proof. We only consider $q>0$. The other case $q<0$ is similar. If $\beta_{+}(t=0) \neq \beta_{-}(t=0)$, then necessarily $\beta_{+}(t=0)=-q$ and $\beta_{-}(t=0)=q$. As both $u(t)$ and $\mathcal{B}_{q} u(t)$ solve NLS, from (4.21), $\frac{z-i \beta_{-}(t)}{z-i \beta_{+}(t)} a(z ; u(t))=a\left(z ; \mathcal{B}_{q} u(t)\right)=a\left(z ; \mathcal{B}_{q} u(t=0)\right)=\frac{z-i \beta_{-}(t=0)}{z-i \beta_{+}(t=0)} a(z ; t=$ $0)=\frac{z-i q}{z+i q} a(z ; u(t=0))=\frac{z-i q}{z+i q} a(z ; u(t))$. Thus, $\beta(t)=-q$ and $\beta_{-}(t)=q$ for all $t \geq 0$ and hence $\tilde{a}(z ; u(t))$ has a simple zero at $z=i q$. As $\gamma\left(i q ; \mathcal{B}_{q} u(t)\right)=\gamma\left(i q ; \mathcal{B}_{q} u(t=0)\right) e^{\frac{i(i q)^{2} t}{2}}$, it follows from (3.36) (3.37) that

$$
\begin{aligned}
-\frac{i q^{2}}{2} & =\frac{\partial}{\partial t} \log (\tilde{\gamma}(i q, t))=\frac{\partial}{\partial t} \log \left(\frac{\left(\psi_{1}^{+}\right)_{2}(0, i q)}{\left(\psi_{2}^{-}\right)_{2}(0, i q)}\right) \\
& =-\frac{i q^{2}}{2}+\left.\frac{i}{2}\left(\bar{u}_{x}+q \bar{u}\right)\right|_{x=0}\left(\frac{\left(\psi_{1}^{+}\right)_{1}}{\left(\psi_{1}^{+}\right)_{2}}-\frac{\left(\psi_{2}^{-}\right)_{1}}{\left(\psi_{2}^{-}\right)_{2}}\right)(0, i q) \\
& =-\frac{i q^{2}}{2}+\left.\frac{i}{2}\left(\bar{u}_{x}+q \bar{u}\right)\right|_{x=0} \frac{a(i q)}{\left(\psi_{1}^{+}\right)_{2}\left(\psi_{2}^{-}\right)_{2}(0, i q)}
\end{aligned}
$$

and hence $u_{x}(0, t)+q u(0, t)=0$. Thus $\left.u(t)\right|_{\mathbb{R}^{+}}$solves $\mathrm{HNLS}_{q}^{+}$.

If $\beta_{+}(t)=\beta_{-}(t)$ for all $t \geq 0$, then from (4.19), we see that either $\left(\psi_{1}^{+}\right)_{2}(0, i q, t)$ or $\left(\psi_{2}^{-}\right)_{2}(0, i q, t)$ is zero for all $t \geq 0$. As $0 \neq$ $a(i q)=\operatorname{det}\left(\psi_{1}^{+}, \psi_{2}^{-}\right)(0, i q, t),\left(\psi_{1}^{+}\right)_{2}(0, i q, t)$ and $\left(\psi_{2}^{-}\right)_{2}(0, i q, t)$ cannot vanish simultaneously. But, these functions are continuous in $t$. Thus, if $\left(\psi_{1}^{+}\right)_{2}(0, i q, t=0)$ (respectively $\left.\left(\psi_{2}^{-}\right)_{2}(0, i q, t=0)\right)$ is zero, then $\left(\psi_{1}^{+}\right)_{2}(0, i q, t)$ (respectively $\left.\left(\psi_{2}^{-}\right)_{2}(0, i q, t)\right)$ is zero for all $t \geq 0$. If $\left(\psi_{1}^{+}\right)_{2}(0, i q, t)=$ 0 for all $t \geq 0$, then $\left(\psi_{1}^{+}\right)_{1}(0, i q, t) \neq 0$ for all $t \geq 0$ and hence it follows from (3.36) that $u_{x}(0, t)+q u(0, t)=0$ for all $t \geq 0$. Similarly, if $\left(\psi_{2}^{-}\right)_{2}(0, i q, t)=0$ for all $t \geq 0$, then from (3.37), $u_{x}(0, t)+q u(0, t)=0$ for all $t \geq 0$. Thus, we conclude that $\left.u(t)\right|_{\mathbb{R}^{+}}$solves $\mathrm{HNLS}_{q}^{+}$.

The question arises whether the Bäcklund extension method described above provides the only way to extend the solution $u^{+}(t)$ of $\operatorname{HNLS}_{q}^{+}$to a solution $u^{e}(t)$ of NLS on the whole line such that $\left.u^{e}(t)\right|_{\mathbb{R}^{+}}=$ 
$u^{+}(t)$. If $v(t)$ is any such extension, we must have

$$
\begin{aligned}
& v(0-, t)=v(0+, t)=u^{+}(0+, t), \\
& v_{x}(0-, t)=v_{x}(0+, t)=u_{x}^{+}(0+, t) .
\end{aligned}
$$

Thus the question reduces to showing that the solution of NLS on $\mathbb{R}^{-}$ is uniquely specified by (4.32). But this is true, as can be seen, for example from the work of Isakov [Isa93]. Thus the Bäcklund extension method is the only way in which one can solve $\mathrm{HNLS}_{q}^{+}$by extension to a solution of NLS on $\mathbb{R}$.

We now develop further properties of the Bäcklund extensions.

Definition 4.16. Let $q \in \mathbb{R}$ and let $u \in L_{\text {loc }}^{1}(\mathbb{R})$. We say that $u$ is q-symmetric if

$$
u=\mathcal{R B}_{q} u
$$

Remark 4.17. Note that if $u(x)$ is $q$-symmetric, then $u$ is the Bäcklund extension of $\left.u\right|_{\mathbb{R}^{+}}$.

Proposition 4.18. The Bäcklund extension of a function $u \in L_{l o c}^{1}(\mathbb{R})$ is q-symmetric.

Proof. By (4.11) and Lemma 4.4(ii),

$$
\left.\left(\mathcal{R B}_{q} u^{e}\right)\right|_{\mathbb{R}^{+}}=\mathcal{R}\left(\left.\left(\mathcal{B}_{q} u^{e}\right)\right|_{\mathbb{R}^{-}}\right)=\mathcal{R}\left(\mathcal{B}_{q}^{-}\left(\left.u^{e}\right|_{\mathbb{R}^{-}}\right)\right)=\mathcal{R B}_{q}^{-} \mathcal{R} \mathcal{B}_{q}^{+} u=u,
$$

and

$$
\left(\mathcal{R} \mathcal{B}_{q} u^{e}\right){\mid \mathbb{R}^{-}}=\mathcal{R}\left(\left.\left(\mathcal{B}_{q} u^{e}\right)\right|_{\mathbb{R}^{+}}\right)=\mathcal{R}\left(\mathcal{B}_{q}^{+}\left(\left.u^{e}\right|_{\mathbb{R}^{+}}\right)\right)=\mathcal{R} \mathcal{B}_{q}^{+} u
$$

Lemma 4.19. Let $u(x) \in H^{1,1}(\mathbb{R})$. If $u$ is q-symmetric, then $\beta \equiv$ $\beta_{+}=\beta_{-}$. In other words, $P(x) \rightarrow-i \beta \sigma_{3}$ as $|x| \rightarrow \infty$ where $\beta^{2}=q^{2}$.

Proof. From the proof of Lemma 4.4, $P(x)=\sigma_{3} P(-x) \sigma_{3}$ and the result follows from Lemma 4.8.

The above calculations together with Proposition 3.1 imply the following.

Proposition 4.20. Suppose that $u(x)$ is q-symmetric. Let $P(x)$ solve (4.1) with $Q(x)=\left(\begin{array}{cc}0 & u(x) \\ -\overline{u(x)} & 0\end{array}\right)$ and let $\psi(x, z)$ be an eigensolution of (3.5). Set $\tilde{\psi}(x, z)=(z+P(x)) \psi(x, z)$. Then,

$$
\hat{\psi}(x, z) \equiv \sigma_{3} \tilde{\psi}(-x,-z)=\sigma_{3}(-z+P(-x)) \psi(-x,-z)
$$

and

$$
\psi^{\#}(x, z) \equiv(-i) \overline{\sigma_{2} \hat{\psi}(x, \bar{z})}=\sigma_{1}(z-\overline{P(-x)}) \overline{\psi(-x,-\bar{z})}
$$


also solve (3.5) where $\sigma_{1}=\left(\begin{array}{ll}0 & 1 \\ 1 & 0\end{array}\right)$ is the first Pauli matrix. Thus the maps $\psi \mapsto \hat{\psi}$ and $\psi \mapsto \psi^{\#}$ are auto-Bäcklund transformations for the equation (3.5). In particular,

$$
\psi^{ \pm}(x, z)=\sigma_{3}(z-P(-x)) \psi^{\mp}(-x,-z)\left(z+i \beta \sigma_{3}\right)^{-1} \sigma_{3}, \quad z \in \mathbb{R}
$$

and

$$
\psi^{ \pm}(x, z)=\sigma_{1}(z-\overline{P(-x)}) \overline{\psi^{\mp}(-x,-z)}\left(z-i \beta \sigma_{3}\right)^{-1} \sigma_{1}, \quad z \in \mathbb{R}
$$

where $\psi^{ \pm}(x, z)$ are the associated $Z S-A K N S$ solutions and $P(x) \rightarrow$ $-i \beta \sigma_{3}$ as $x \rightarrow \pm \infty$.

Remark 4.21. As $\psi_{1}^{+}(x, z), \psi_{2}^{-}(x, z)$ continue analytically into $\mathbb{C}^{+}$, for $z \in \mathbb{C}^{+} \backslash\{-i \beta\}$

$$
\psi_{1}^{+}(x, z)=\frac{1}{z+i \beta} \sigma_{1}(z-\overline{P(-x)}) \overline{\psi_{2}^{-}(-x,-\bar{z})},
$$

and for $z \in \mathbb{C}^{+} \backslash\{i \beta\}$,

$$
\psi_{2}^{-}(x, z)=\frac{1}{z-i \beta} \sigma_{1}(z-\overline{P(-x)}) \overline{\psi_{1}^{+}(-x,-\bar{z})} .
$$

If $\beta>0$, say, it is easy to check that the apparent singularity in (4.37) as $z \rightarrow i \beta$, is in fact removable, as it should be, etc.

Lemma 4.22. Suppose that $u(x) \in H^{1,1}(\mathbb{R})$ is q-symmetric. Then, the scattering function $a(z)$ of $u(x)$ does not vanish at $z=i|q|$.

Proof. Let $\psi_{1}^{+}, \psi_{2}^{-}$be the associated ZS-AKNS solutions and let $P(x)$ solve (4.1). By Lemma4.19, $P(x) \rightarrow-i \beta \sigma_{3}$ as $x \rightarrow \pm \infty$ where $\beta^{2}=q^{2}$. Suppose first that $\beta=-|q|<0$. If $a(i|q|)=0$, necessarily $\psi_{1}^{+}(x, i|q|)=$ $\gamma \psi_{2}^{-}(x, i|q|)$ for some constant $\gamma \neq 0$. But then, it follows from (4.37) that

$$
\psi_{1}^{+}(0, i|q|)=\gamma \psi_{2}^{-}(x, i|q|)=\frac{\gamma}{2 i|q|} \sigma_{1}\left(i|q|-i q \sigma_{3}\right) \overline{\psi_{1}^{+}(0, i|q|)} .
$$

As $i|q|-i q \sigma_{3}$ is either $\left(\begin{array}{cc}0 & 0 \\ 0 & 2 i q\end{array}\right)$ or $\left(\begin{array}{rr}-2 i q & 0 \\ 0 & 0\end{array}\right)$, we conclude that $\psi_{1}^{+}(0, i|q|)$ $=0$, which is a contradiction. If $\beta_{+}=|q|>0$, we utilize (4.36) and the proof is similar.

Remark 4.23. It follows by Proposition 4.18 and Lemma 4.22 that the scattering function $a(z)$ of any Bäcklund extension of $u \in H^{1,1}\left(\mathbb{R}^{+}\right)$ does not vanish at $z=i|q|$.

$\mathcal{B}_{q}, \mathcal{B}_{q}^{ \pm}$are not continuous in $H^{1,1}(\mathbb{R}), H^{1,1}\left(\mathbb{R}^{ \pm}\right)$, respectively. In other words, a small perturbation of $u(x)$ may not result in a small perturbation of the Bäcklund transformation $\tilde{u}(x)$. For example, consider the case when $q>0$ and $u(x)=0$ on $\mathbb{R}^{+}$. Let $f_{\lambda}(x)=v_{\lambda}(x)$, 
$x \geq 0$, where $v_{\lambda}$ is defined by (1.6). Then, $\left\|f_{\lambda}\right\|_{H^{1,1}\left(\mathbb{R}^{+}\right)} \rightarrow 0$ as $\lambda \downarrow q$. From Remark 4.33, $\left\|\widetilde{f}_{\lambda}\right\|_{H^{1,1}\left(\mathbb{R}^{+}\right)}=\left\|\eta_{\lambda}\right\|_{H^{1,1}\left(\mathbb{R}^{-}\right)}$, but $\left\|\eta_{\lambda}\right\|_{H^{1,1}\left(\mathbb{R}^{-}\right)}$is not small for any $\lambda>q>0$. In the case when $q<0$ and $u(x)=v_{\mu} \mid \mathbb{R}^{+}$, there is a sequence of 2 -solitons with symmetry conditions which converges to $v_{\mu}$ on $\mathbb{R}^{+}$.

The following lemma, however, shows that $\mathcal{B}_{q}, \mathcal{B}_{q}^{ \pm}$are continuous at $u \in H^{1,1}\left(\mathbb{R}^{+}\right)$for which $g_{2}(0, i q ; u) \neq 0$, where $g(x, z)=\left(g_{1}, g_{2}\right)^{T}$, $x \geq 0, z \in \overline{\mathbb{C}^{+}}$be as in the proof of Proposition 4.7.

Lemma 4.24. Let $q>0$ be given. Let $\left\{u_{n}\right\}$ be any sequence in $H^{1,1}\left(\mathbb{R}^{+}\right)$such that $u_{n} \rightarrow u$ as $n \rightarrow \infty$. If $g_{2}(0, i q) \neq 0$, then $\mathcal{R} \mathcal{B}_{q}^{+} u_{n} \rightarrow$ $\mathcal{R} \mathcal{B}_{q}^{+} u$. In particular, $u_{n}^{e} \rightarrow u^{e}$ where $u_{n}^{e}$, $u^{e}$ are the Bäcklund extensions of $u_{n}, u$, respectively.

Proof. Note that $g(x, z ; u)=\psi_{1}^{+}\left(x, z ; u^{e}\right), x \geq 0$. From Remark 4.23, we have $a\left(i q ; u^{e}\right) \neq 0$, and hence $\Psi_{1}^{0}\left(x, i q ; u^{e}\right)=c_{1}\left(u^{e}\right) \psi_{1}^{+}\left(x, i q ; u^{e}\right)+$ $c_{2}\left(u^{e}\right) \psi_{2}^{-}\left(x, i q ; u^{e}\right)$, where $c_{1}\left(u^{e}\right)=\left(\psi_{2}^{-}\right)_{2}\left(0, i q ; u^{e}\right) / a\left(i q ; u^{e}\right), c_{2}\left(u^{e}\right)=$ $-\left(\psi_{1}^{+}\right)_{2}\left(0, i q ; u^{e}\right) / a\left(i q ; u^{e}\right)$. Note that $c_{2}\left(u^{e}\right)$ is non-zero. Let $\xi_{n}(x)=$ $\left(\left(\xi_{n}\right)_{1},\left(\xi_{n}\right)_{2}\right)^{T}=\Psi_{1}^{0}\left(x, i q ; u_{n}^{e}\right) e^{-q x / 2}$ and $\xi(x)=\left(\xi_{1}, \xi_{2}\right)^{T}=\Psi_{1}^{0}\left(x, i q ; u^{e}\right) e^{-q x / 2}$.

From (4.5), we see that

$$
\mathcal{B}_{q}^{+} u_{n}=u_{n}-2 q \frac{\left(\xi_{n}\right)_{1} \overline{\left(\xi_{n}\right)_{2}}}{\left|\left(\xi_{n}\right)_{1}\right|^{2}+\left|\left(\xi_{n}\right)_{2}\right|^{2}}
$$

and

$$
\mathcal{B}_{q}^{+} u=u-2 q \frac{\xi_{1} \overline{\xi_{2}}}{\left|\xi_{1}\right|^{2}+\left|\xi_{2}\right|^{2}}
$$

By Lemma 7.1, we have $a\left(i q ; u_{n}^{e}\right) \rightarrow a\left(i q ; u^{e}\right)$ and hence $c_{1}\left(u_{n}^{e}\right) \rightarrow$ $c_{1}\left(u^{e}\right), c_{2}\left(u_{n}^{e}\right) \rightarrow c_{2}\left(u^{e}\right)$. Set $m_{2}^{-}(x, z)=\left(\left(m_{2}^{-}\right)_{1},\left(m_{2}^{-}\right)_{2}\right)^{T} \equiv \psi_{2}^{-}(x, z) e^{i x z / 2}$. Again by Lemma 7.1, we have $\psi_{1}^{+}\left(x, i q ; u_{n}^{e}\right) \rightarrow \psi_{1}^{+}\left(x, i q ; u^{e}\right)$ in $H^{1,1}\left(\mathbb{R}^{+}\right)$, $\left(m_{2}^{-}\right)_{1}\left(x, i q ; u_{n}^{e}\right) \rightarrow\left(m_{2}^{-}\right)_{1}\left(x, i q ; u^{e}\right)$ in $H^{1,1}\left(\mathbb{R}^{+}\right),\left(m_{2}^{-}\right)_{2}\left(x, i q ; u_{n}^{e}\right) \rightarrow$ $\left(m_{2}^{-}\right)_{2}\left(x, i q ; u^{e}\right)$ in $L^{\infty}\left(\mathbb{R}^{+}\right)$, and $\partial_{x}\left(m_{2}^{-}\right)_{2}\left(x, i q ; u_{n}^{e}\right) \rightarrow \partial_{x}\left(m_{2}^{-}\right)_{2}\left(x, i q ; u^{e}\right)$ in $L^{2}\left(\mathbb{R}^{+}\right)$, As $\left|\xi_{1}(x)\right|^{2}+\left|\xi_{2}(x)\right|^{2} \geq c>0$ for all $x \geq 0$, we conclude that $\mathcal{B}_{q}^{+} u_{n} \rightarrow \mathcal{B}_{q}^{+} u$ in $H^{1,1}\left(\mathbb{R}^{+}\right)$.

The following fact is basic.

Proposition 4.25. If $u \in C^{1}(\mathbb{R})$ is q-symmetric, then

$$
u^{\prime}(0)+q u(0)=0 .
$$

In other words, q-symmetry yields the boundary condition. 
Proof. Observe that $\tilde{Q}(0)=Q(0)-i[\sigma, P(0)]=Q(0)$ and

$$
\begin{aligned}
\tilde{Q}_{x}(0)+Q_{x}(0) & =2 Q_{x}(0)-i\left[\sigma, P_{x}(0)\right] \\
& =2 Q_{x}(0)-i[\sigma, \tilde{Q}(0) P(0)-P(0) Q(0)] \\
& =2 Q_{x}(0)-i\left[\sigma, i q\left[\sigma_{3}, Q(0)\right]\right] \\
& =2\left(Q_{x}(0)+q Q(0)\right) .
\end{aligned}
$$

As $\tilde{Q}_{x}(0)=-Q_{x}(0)$ by the $q$-symmetry condition, the equation (4.38) follows.

Proposition 4.26. If $u \in C^{2}\left(\mathbb{R}^{+}\right)$and $u^{\prime}(0+)+q u(0)=0$, then the Bäcklund extension of $u$ belongs to $C^{2}(\mathbb{R})$.

Proof. From the proof of Proposition 4.25, we have $u^{e}(0-)=u^{e}(0+)$ and $\left(u^{e}\right)^{\prime}(0-)=\left(u^{e}\right)^{\prime}(0+)$. For $u^{\prime \prime}(0)$,

$$
\begin{aligned}
\partial_{x}^{2} Q^{e}(0-)-\partial_{x}^{2} Q^{e}(0+) & =\partial_{x}^{2} \tilde{Q}(0)-\partial_{x}^{2} Q(0) \\
& =-i\left[\sigma, \partial_{x}^{2} P\right](0) \\
& =-i\left[\sigma, \tilde{Q}_{x} P-P Q_{x}+\tilde{Q} P_{x}-P_{x} Q\right](0) \\
& =-i\left[\sigma,-Q_{x} P-P Q_{x}-\left[P_{x}, Q\right](0)\right. \\
& =-i\left[\sigma,-i q\left[\left[\sigma_{3}, Q(0)\right], Q(0)\right]=0 .\right.
\end{aligned}
$$

We need the following lemma to prove Proposition 4.28 below.

Lemma 4.27. Let $\Psi(x, z)$ be any invertible solution to (3.5) for $z \in \mathbb{R}$. Then,

$$
S(z)=\lim _{x \rightarrow \infty} e^{i x z \sigma} \Psi(-x, z) \Psi^{-1}(x, z) e^{i x z \sigma} .
$$

Proof. Let $\psi^{ \pm}=\left(\psi_{1}^{ \pm}, \psi_{2}^{ \pm}\right)$be the ZS-AKNS solutions normalized at $\pm \infty$, respectively. As $\psi^{+}$solves (3.5) and is invertible, $\Psi(x, z)=$ $\psi^{+}(x, z) M(z)$ for some invertible matrix $M(z)$.

$$
\begin{aligned}
& \lim _{x \rightarrow \infty} e^{i x z \sigma} \Psi(-x, z) \Psi^{-1}(x, z) e^{i x z \sigma} \\
& \quad=\lim _{x \rightarrow \infty} e^{i x z \sigma} \psi^{+}(-x, z)\left(\psi^{+}(x, z)\right)^{-1} e^{i x z \sigma} \\
& \quad=\lim _{x \rightarrow \infty} e^{i x z \sigma} \psi^{-}(-x, z) S(z)\left(\psi^{+}(x, z)\right)^{-1} e^{i x z \sigma}
\end{aligned}
$$

Since $\psi^{-}(-x, z) e^{i x z \sigma}, \psi^{+}(x, z) e^{-i x z \sigma} \rightarrow I$ as $x \rightarrow \infty$, we obtain the result.

For $u \in H^{1,1}(\mathbb{R})$, the $q$-symmetry condition (4.33) can be reformulated in terms of the scattering data of $u$ as follows. 
Proposition 4.28. Let $q \in \mathbb{R} \backslash\{0\}$ be given. Suppose that $u \in \mathcal{G} \subset$ $H^{1,1}(\mathbb{R})$ is generic with scattering data $a(z), b(z), Z_{+}=\left\{z_{1}, \cdots, z_{n}\right\}$, $\Gamma_{+}=\left\{\gamma\left(z_{1}\right), \cdots, \gamma\left(z_{n}\right)\right\}$. Then, $u$ is q-symmetric if and only if

$$
\left\{\begin{array}{l}
\overline{a(-\bar{z})}=a(z), \quad z \in \overline{\mathbb{C}^{+}} \\
b(-z)=b(z) \frac{z+i \beta}{z-i \beta}, \quad z \in \mathbb{R}, \\
z_{k} \neq \pm i \beta \text { and } \gamma\left(z_{k}\right) \overline{\gamma\left(-\overline{z_{k}}\right)}=\frac{z_{k}-i \beta}{z_{k}+i \beta}, \quad k=1, \ldots, n, \\
\beta=(-1)^{n} q .
\end{array}\right.
$$

Remark 4.29. Note that if $u$ is $q$-symmetric, then $a(i|q|) \neq 0$ by Lemma 4.22. Hence $z_{k} \neq \pm i \beta$. For if $z_{k}= \pm i \beta$ then $z_{k}^{2}=-\beta^{2}=-q^{2}$ and so $z_{k}=i|q|$, which contradicts $a(i|q|) \neq 0$.

Remark 4.30. For $z \in \mathbb{R}$, we have

$$
|r(z)|=\left|\frac{b(z)}{a(z)}\right|=\left|\frac{b(-z)}{a(-z)}\right|=|r(-z)| .
$$

By setting $z=0$ in the symmetry condition for $b(z)$, it follows that $b(0)=0$ and hence $r(0)=0$.

Proof. Suppose first that $u(x)$ is $q$-symmetric. From (4.35), it follows that for $z \in \mathbb{R}$,

$$
\begin{aligned}
S(z) & =\left(\psi^{-}\right)^{-1} \psi^{+}(x, z) \\
& =\sigma_{1}\left(z-i \beta \sigma_{3}\right) \overline{\left(\psi^{+}\right)^{-1} \psi^{-}(-x,-z)}\left(z-i \beta \sigma_{3}\right)^{-1} \sigma_{1} \\
& =\sigma_{1}\left(z-i \beta \sigma_{3}\right) \overline{S^{-1}(-z)}\left(z-i \beta \sigma_{3}\right)^{-1} \sigma_{1} .
\end{aligned}
$$

As $S(z)=\left(\begin{array}{cc}a(z) & -\overline{b(z)} \\ b(z) & \overline{a(z)}\end{array}\right)$, we conclude that $a(z)=\overline{a(-z)}$ and $b(-z)=$ $b(z) \frac{z+i \beta}{z-i \beta}, z \in \mathbb{R}$ and hence by analytic continuation,

$$
a(z)=\overline{a(-\bar{z})}, \quad z \in \overline{\mathbb{C}^{+}} \text {and } b(-z)=b(z) \frac{z+i \beta}{z-i \beta}, \quad z \in \mathbb{R} .
$$

Let $\Psi^{0}(x, z)$ be the solution of (3.5) with $\Psi^{0}(0, z)=I$. By Proposition 4.20, there exists an $M_{0}(z)$ such that $\sigma_{3}(-z+P(-x)) \Psi^{0}(-x,-z)=$ $\Psi^{0}(x, z) M_{0}(z)$. Setting $x=0, z=0$, we obtain $M_{0}(0)=-i q I$ and hence $\sigma_{3} P(-x) \Psi^{0}(-x, 0)=-i q \Psi^{0}(x, 0)$. Thus, by Lemma 4.27,

$$
S(0)=\lim _{x \rightarrow \infty} \Psi^{0}(-x, 0)\left(\Psi^{0}\right)^{-1}(x, 0)=\lim _{x \rightarrow \infty}-i q\left(\sigma_{3} P(-x)\right)^{-1}=\frac{q}{\beta} I
$$

On the other hand, from (4.41), $a(0)=\overline{a(0)}, b(0)=0$ and so $S(0)=$ $a(0) I$. Note that $a(z)$ has the explicit formula (3.24) (recall $|r|^{2}=$ 
$\left.|b|^{2} /|a|^{2},|a|^{2}+|b|^{2}=1\right)$,

$$
a(z)=\prod_{k=1}^{n} \frac{z-z_{k}}{z-\overline{z_{k}}} \exp \left[\frac{1}{2 \pi i} \int_{\mathbb{R}} \frac{\log \left(1-|b(s)|^{2}\right)}{s-z} \mathrm{~d} s\right], \quad z \in \mathbb{C}^{+} .
$$

Using this formula together with $b(0)=0$ and $|b(-z)|=|b(z)|$ from (4.41), we see that $a(0)=\prod_{k=1}^{n} z_{k} / \prod_{k=1}^{n} \overline{z_{k}}$. In computing $a(0)=$ $\lim _{z \rightarrow 0, z \in \mathbb{C}^{+}} a(z)$ via (4.42), we have used the fact that $b(z) \in H^{1,1} \subset$ $H^{1,0}$ (see (3.26) $)$ and so $|b(z)|^{2}=O(|z|)$ as $z \rightarrow 0, z \in \mathbb{R}$. As $a\left(z_{k}\right)=0$ if and only if $a\left(-\overline{z_{k}}\right)=0, a(0)=\prod_{k=1}^{n}\left(-\overline{z_{k}}\right) / \prod_{k=1}^{n} \overline{z_{k}}=(-1)^{n}$. Thus, $\beta=q / a(0)=(-1)^{n} q$. Now using (4.37), we see that

$$
\begin{aligned}
\psi_{1}^{+}\left(x, z_{k}\right) & =\gamma\left(z_{k}\right) \psi_{2}^{-}\left(x, z_{k}\right) \\
& =\frac{\gamma\left(z_{k}\right)}{z_{k}-i \beta} \sigma_{1}\left(z_{k}-\overline{P(-x)}\right) \overline{\psi_{1}^{+}\left(-x,-\overline{z_{k}}\right)} \\
& =\frac{\gamma\left(z_{k}\right) \overline{\gamma\left(-\overline{z_{k}}\right)}}{z_{k}-i \beta} \sigma_{1}\left(z_{k}-\overline{P(-x)}\right) \overline{\psi_{2}^{-}\left(-x,-\overline{z_{k}}\right)}
\end{aligned}
$$

Comparing with (4.36), we obtain $\gamma\left(z_{k}\right) \overline{\gamma\left(-\overline{z_{k}}\right)}=\frac{z_{k}-i \beta}{z_{k}+i \beta}$.

Conversely, suppose that the scattering data for $u(x)$ satisfy the symmetries (4.39). Denote by $m(x, z)=\left(m_{1}(x, z), m_{2}(x, z)\right)$ the solution to the normalized RHP with the jump matrix

$$
v_{x}(z)=\left(\begin{array}{cc}
1+\left|r_{x}(z)\right|^{2} & r_{x}(z) \\
r_{x}(z) & 1
\end{array}\right), \quad r_{x}(z) \equiv r(z) e^{i x z}
$$

Define

$$
\mathfrak{b}(x)=\left(m_{1}(x, i \beta), m_{2}(x,-i \beta)\right) .
$$

From the relation in Proposition 3.1(i), we see that $m_{2}(x,-i \beta)=$ $i \sigma_{2} \overline{m_{1}(x, i \beta)}$. Hence $\operatorname{det} b(x)=\left|m_{1}(x, i \beta)\right|^{2}>0$ and so $b(x)$ is invertible. Set

$$
U_{0}=-i \beta \sigma_{3}, U(x)=\mathfrak{b}(x) U_{0} \mathfrak{b}(x)^{-1}
$$

and define

$\breve{m}(x, z) \equiv\left\{\begin{array}{l}\sigma_{1}(z-U(x))^{-1} m(x, z)\left(z-U_{0}\right) a(z)^{\sigma_{3}} \sigma_{1}, \quad z \in \mathbb{C}^{+} \backslash\{i|q|\}, \\ \sigma_{1}(z-U(x))^{-1} m(x, z)\left(z-U_{0}\right) \overline{a(\bar{z})}{ }^{-\sigma_{3}} \sigma_{1}, z \in \mathbb{C}^{-} \backslash\{-i|q|\} .\end{array}\right.$

Claim: $m(x, z)=\overline{\breve{m}(-x,-\bar{z})}$ 
We prove this claim in a number of steps. First, for $z \in \mathbb{R}$,

$$
\begin{aligned}
\left(\breve{m}_{-}\right)^{-1} \breve{m}_{+}(x, z) & =\sigma_{1} \overline{a(z)} \sigma^{\sigma_{3}}\left(z-U_{0}\right)^{-1} v_{x}(z)\left(z-U_{0}\right) a(z)^{\sigma_{3}} \sigma_{1} \\
& =\sigma_{1}\left(\begin{array}{cc}
1 & r_{x}(z) \frac{z-i \beta}{z+i \beta} \overline{a(z)} \\
\hline r_{x}(z) \frac{z+i \beta}{z-i \beta} \frac{a(z)}{a(z)} & 1+\left|r_{x}(z)\right|^{2}
\end{array}\right) \sigma_{1} \\
& =\left(\begin{array}{cc}
1+\left|r_{-x}(-z)\right|^{2} & \overline{r_{-x}(-z)} \\
r_{-x}(-z) & 1
\end{array}\right) .
\end{aligned}
$$

Here, we used the symmetries (4.39) for the scattering data. Set $m^{\#}(x, z) \equiv \overline{\breve{m}(-x,-\bar{z})}$. Then,

$$
\left(m_{-}^{\#}\right)^{-1} m^{\#}+(x, z)=\overline{\left(\breve{m}_{-}\right)^{-1} \breve{m}_{+}(-x,-z)}=v_{x}(z) .
$$

Observe that for $z \in \mathbb{C} \backslash(\mathbb{R} \cup\{ \pm i|q|\}$,

$$
(z-U(x))^{-1} m(x, z)\left(z-U_{0}\right)=\mathfrak{b}\left(\begin{array}{cc}
\left(\mathfrak{b}^{-1} m\right)_{11} & \left(\mathfrak{b}^{-1} m\right)_{12} \frac{z-i \beta}{z+i \beta} \\
\left(\mathfrak{b}^{-1} m\right)_{21} \frac{z+i \beta}{z-i \beta} & \left(\mathfrak{b}^{-1} m\right)_{22}
\end{array}\right) .
$$

Since $\left(\mathfrak{b}^{-1} m\right)_{21}(x, i \beta)=0$ and $\left(\mathfrak{b}^{-1} m\right)_{12}(x,-i \beta)=0$ for $x \in \mathbb{R}, m^{\#}(x, z)$ does not have a pole at $z= \pm i \beta$ in the $z$-plane. As $u \in \mathcal{G}$, for any pole $z_{k} \in \mathbb{C}^{+}$

$$
\operatorname{Res}_{z=z_{k}} m(x, z)=\lim _{z \rightarrow z_{k}}\left(z-z_{k}\right) m(x, z)=\lim _{z \rightarrow z_{k}} m(x, z)\left(\begin{array}{cc}
0 & 0 \\
c_{x}\left(z_{k}\right) & 0
\end{array}\right) .
$$

where $c_{x}\left(z_{k}\right)=e^{-i x z_{k}} c\left(z_{k}\right)$ and $c\left(z_{k}\right)=\frac{\gamma\left(z_{k}\right)}{a^{\prime}\left(z_{k}\right)}$. Equivalently,

$$
\lim _{z \rightarrow z_{k}} a(z) m_{1}(x, z)=a^{\prime}\left(z_{k}\right) m_{2}\left(x, z_{k}\right) c_{x}\left(z_{k}\right)=\gamma\left(z_{k}\right) m_{2}\left(x, z_{k}\right) e^{-i x z_{k}}
$$

For $m^{\#}(x, z)$, we have

$$
\begin{aligned}
& \overline{\operatorname{Res}_{z=-\overline{z_{k}}} m^{\#}(-x, z)} \\
& =\lim _{z \rightarrow-\bar{z}_{k}} \overline{\left(z-\left(-\overline{z_{k}}\right) m^{\#}(-x, z)\right.}=\lim _{z \rightarrow z_{k}}-\left(z-z_{k}\right) \overline{m^{\#}(-x,-\bar{z})} \\
& =\lim _{z \rightarrow z_{k}}-\sigma_{1}(z-U(x))^{-1}\left(z-z_{k}\right) m(x, z) a(z)^{\sigma_{3}}\left(z-U_{0}\right) \sigma_{1} \\
& =\lim _{z \rightarrow z_{k}}-\sigma_{1}(z-U(x))^{-1}\left(z-z_{k}\right)\left(a(z) m_{1}(x, z) \frac{m_{2}(x, z)}{a(z)}\right)\left(z-U_{0}\right) \sigma_{1} \\
& =-\sigma_{1}\left(z_{k}-U(x)\right)^{-1}\left(\frac{z_{k}-i \beta}{a^{\prime}\left(z_{k}\right)} m_{2}\left(x, z_{k}\right) \quad 0\right)
\end{aligned}
$$

Thus at $-\overline{z_{k}}$, the second column of $m^{\#}(x, z)$ is analytic, and the first column has a simple pole. A similar calculation shows that at $-z_{k}$, 
the first column of $m^{\#}(x, z)$ is analytic, and the second column has a simple pole. On the other hand, if we set $c_{x}^{\#}\left(z_{k}\right)=e^{-i x z_{k}} c^{\#}\left(z_{k}\right)$, then

$$
\begin{aligned}
& \lim _{z \rightarrow-\overline{z_{k}}} \overline{m^{\#}(-x, z)\left(\begin{array}{cc}
0 & 0 \\
c_{-x}^{\#}\left(-\overline{z_{k}}\right) & 0
\end{array}\right)}=\lim _{z \rightarrow z_{k}} \overline{m^{\#}(-x,-\bar{z})}\left(\begin{array}{cc}
0 & 0 \\
\overline{c_{-x}^{\#}\left(-\overline{z_{k}}\right)} & 0
\end{array}\right) \\
& =\lim _{z \rightarrow z_{k}} \sigma_{1}(z-U(x))^{-1} m(x, z) a(z)^{\sigma_{3}}\left(z-U_{0}\right) \sigma_{1}\left(\begin{array}{cc}
0 & 0 \\
c_{-x}^{\#}\left(-\overline{z_{k}}\right) & 0
\end{array}\right) \\
& =\lim _{z \rightarrow z_{k}} \sigma_{1}(z-U(x))^{-1}\left(\overline{c_{-x}^{\#}\left(-\overline{z_{k}}\right)}(z+i \beta) a(z) m_{1}(x, z) \quad 0\right) \\
& =\sigma_{1}\left(z_{k}-U(x)\right)^{-1}\left(\overline{c^{\#}\left(-\overline{z_{k}}\right)}\left(z_{k}+i \beta\right) \gamma\left(z_{k}\right) m_{2}\left(x, z_{k}\right) \quad 0\right),
\end{aligned}
$$

Here we have used (4.46). Comparing with (4.47) and using (4.39), we have

$$
c^{\#}\left(-\overline{z_{k}}\right)=-\overline{\frac{1}{a^{\prime}\left(z_{k}\right) \gamma\left(z_{k}\right)} \frac{z_{k}-i \beta}{z_{k}+i \beta}}=-\frac{\gamma\left(-\overline{z_{k}}\right)}{\overline{a^{\prime}\left(z_{k}\right)}} .
$$

As $a(z)=\overline{a(-\bar{z})}, \frac{a(z)}{z-z_{k}}=-\overline{\left(\frac{a(-\bar{z})}{-\bar{z}-\left(-\overline{z_{k}}\right)}\right)}$ and so $a^{\prime}\left(z_{k}\right)=-\overline{a^{\prime}\left(-\overline{z_{k}}\right)}$. Hence it follows that $c^{\#}\left(-\overline{z_{k}}\right)=\frac{\gamma\left(-\overline{z_{k}}\right)}{a^{\prime}\left(-\overline{z_{k}}\right)}=c\left(-\overline{z_{k}}\right)$ and so $c^{\#}\left(z_{k}\right)=c\left(z_{k}\right)$ for $k=1, \cdots, n$. Assembling the above results, we conclude by the uniqueness for the RHP that $m^{\#}(x, z)=m(x, z)$, which proves the above Claim.

Now fix $x=0$ and write $\mathfrak{b}=\mathfrak{b}(0)$ and $m(z)=m(0, z)$, etc. If $q>0$ and $n$ is even, then $i \beta \in \mathbb{C}^{+}$and

$$
\begin{aligned}
\overline{m_{21}(i \beta)} & =\overline{m_{21}^{\#}(i \beta)}=\breve{m}_{21}(i \beta) \\
& =\lim _{z \rightarrow i \beta}\left(\mathfrak{b}\left(z-U_{0}\right)^{-1} \mathfrak{b}^{-1} m(z)\left(z-U_{0}\right) a(z)^{\sigma_{3}}\right)_{12} \\
& =\frac{\mathfrak{b}_{12}}{a(i \beta)}\left(\mathfrak{b}^{-1} m\right)_{22}(i \beta) \\
& =\frac{m_{12}(-i \beta)}{a(i \beta)} \frac{1}{\operatorname{det} \mathfrak{b}}\left(-m_{21} m_{12}+m_{11} m_{22}\right)(i \beta) \\
& =-\frac{\frac{m_{21}(i \beta)}{a(i \beta) \operatorname{det} \mathfrak{b}}}{}
\end{aligned}
$$

Let $Z_{+}^{(1)}=Z_{+} \cap i \mathbb{R}$ and $Z_{+}^{(2)}=Z_{+} \cap\left\{z \in \mathbb{C}^{+}: \operatorname{Re} z>0\right\}$. If $z_{k}=$ $i \xi \in Z_{+}^{(1)}$, then by the symmetries (4.39), $0<|\gamma(i \xi)|^{2}=\frac{\xi-\beta}{\xi+\beta}$ and so $\frac{i \beta-i \xi}{i \beta+i \xi}<0$. If $z_{k} \in Z_{+}^{(2)}$, then $-\overline{z_{k}} \in Z_{+}$and $\frac{i \beta-z_{k}}{i \beta-\overline{z_{k}}} \frac{i \beta-\left(-\overline{z_{k}}\right)}{i \beta-\overline{\left(\overline{z_{k}}\right)}}=\left|\frac{i \beta-z_{k}}{i \beta-\overline{z_{k}}}\right|^{2}>0$. Since $n$ is even, $\left|Z_{+}^{(1)}\right|$ is even and hence we see that $\prod_{k=1}^{n} \frac{i \beta-z_{k}}{i \beta-\overline{z_{k}}}>0$. On the other hand, as $|b(-z)|=|b(z)|, z \in \mathbb{R}$, we see $\int_{\mathbb{R}} \frac{\log \left(1-|b(s)|^{2}\right)}{s-i \beta} \mathrm{d} s \in i \mathbb{R}$, 
so it follows from the formula (4.42) that $a(i \beta)>0$. Since $\operatorname{det} \mathfrak{b}=$ $\left|m_{1}(i \beta)\right|^{2}>0$, it follows that

$$
m_{21}(0, i q)=m_{21}(0, i \beta)=0 .
$$

It follows in a similar way that

$$
\begin{aligned}
& m_{21}(0, i q)=m_{21}(0, i \beta)=0, \quad \text { if } q<0, \quad n \text { is even, } \\
& m_{11}(0,-i q)=m_{11}(0, i \beta)=0, \quad \text { if } q>0, \quad n \text { is odd, } \\
& m_{11}(0,-i q)=m_{11}(0, i \beta)=0, \quad \text { if } q<0, \quad n \text { is odd. }
\end{aligned}
$$

Set $\psi(x, z) \equiv m(x, z) e^{i x z \sigma}$. If $\mathrm{n}$ is even,

$$
\Psi_{1}^{0}(x, i q)=\psi(x, i q) \psi(0, i q)^{-1} e_{1}=c_{1} \psi_{1}(x, i q)=c_{1} \psi_{1}(x, i \beta),
$$

for some nonzero constant $c_{1}$. If $\mathrm{n}$ is odd,

$$
\Psi_{2}^{0}(x,-i q)=\psi(x,-i q) \psi(0,-i q)^{-1} e_{2}=c_{2} \psi_{1}(x,-i q)=c_{2} \psi_{1}(x, i \beta),
$$

for some nonzero constant $c_{2}$. Thus, using the symmetries $\Psi_{2}^{0}(x, \bar{z})=$ $i \sigma_{2} \overline{\Psi_{1}^{0}(x, z)}$, etc., we obtain

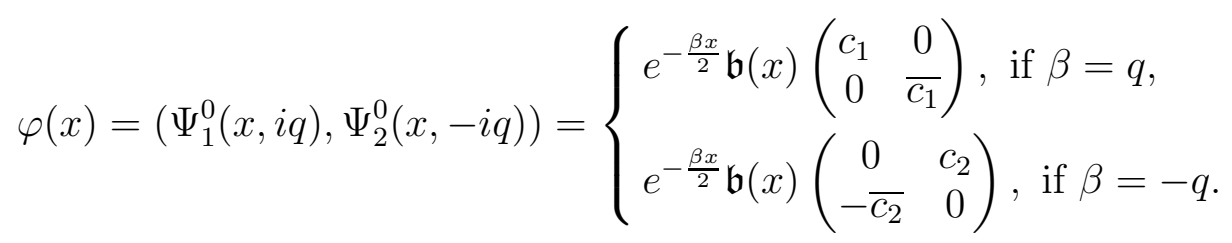

In both cases we see that

$$
U(x)=\mathfrak{b}(x) U_{0} \mathfrak{b}(x)^{-1}=\varphi(x)\left(-i q \sigma_{3}\right) \varphi(x)^{-1}=P(x) .
$$

where $P(x)$ solves (4.1). Let $m(x, z)=I+\frac{m_{1}(x)}{z}+o\left(\frac{1}{z}\right)$ and $a(z)=$ $1+\frac{a_{1}}{z}+o\left(\frac{1}{z}\right)$ be the expansions as $z \rightarrow \infty,|\operatorname{Im} z|>c|\operatorname{Re} z|, c>0$. As $\breve{m}(x, z)=\overline{m(-x,-\bar{z})}$, it follows from (4.45) that

$$
-\overline{m_{1}(-x)}=\sigma_{1}\left(P(x)+m_{1}(x)+a_{1} \sigma_{3}-U_{0}\right) \sigma_{1} .
$$

From the symmetries $m(x, z)=\overline{\sigma_{2} m(x, \bar{z}) \sigma_{2}}, P(x)=\overline{\sigma_{2} P(x) \sigma_{2}}$, we see that $\left(m_{1}\right)_{12}(x)=-\overline{\left(m_{1}\right)_{21}(x)}$ and $P_{12}(x)=-\overline{P_{21}(x)}$. Hence, it follows that

$$
\begin{aligned}
u(x) & =-i\left(m_{1}\right)_{12}(x)=i\left(\overline{\sigma_{1}\left(P(-x)+m_{1}(-x)+a_{1} \sigma_{3}-U_{0}\right) \sigma_{1}}\right)_{12} \\
& =i\left(\overline{P(-x)+m_{1}(-x)}\right)_{21}=-i\left(m_{1}\right)_{12}(-x)-i P_{12}(-x) \\
& =u(-x)-i P_{12}(-x)=\tilde{u}(-x),
\end{aligned}
$$

which completes the proof. 
Remark 4.31. Suppose we are in a situation when the reflection coefficient $r(z)=\frac{\bar{b}}{\bar{a}}$ is small, as is the case corresponding to our data $u_{0}=v_{\mu}+\epsilon w$ (note that for $\epsilon=0, u_{0}=v_{\mu}$ gives rise to a solution with $r \equiv 0$ ). The solution of the RHP $m=\left(m_{i j}\right)_{1 \leq i, j \leq 2}$ is given by (see (3.35)

$$
m(x, z)=I+\frac{1}{2 \pi i} \int_{\mathbb{R}} \frac{\mu_{x}(s)\left(v_{x}(s)-I\right)}{s-z} \mathrm{~d} s+\text { polar terms. }
$$

where $\mu_{x}$ solves (3.34). For $r$ small, we see that $\mu \sim I+\nu$ where $\nu$ is a sum of polar terms. Substituting $\mu_{x} \sim I+\nu$ into (4.48), we obtain

$$
\begin{aligned}
u(x) & =-i \lim _{z \rightarrow \infty} z m_{12}(x, z) \\
& =i \int_{\mathbb{R}} \mu_{x}\left(v_{x}-I\right) \frac{\mathrm{d} z}{2 \pi i}+\text { polar terms } \\
& \sim \frac{1}{2 \pi} \int_{\mathbb{R}} r(z) e^{i\left(x z-z^{2} t / 2\right)}+\text { polar terms }
\end{aligned}
$$

Here, as $|a|^{2}=1-|b|^{2}, r=\frac{\bar{b}}{\bar{a}} \sim \bar{b}$. Neglecting the polar contribution we see that this formula is of the same form as formula (1.31) for the solution in the linear case $u_{e}(x, t)=\frac{1}{2 \pi} \int_{\mathbb{R}} e^{i\left(x z-t z^{2} / 2\right)} \hat{u}(z) \mathrm{d} z$. Here $(i z+$ q) $\hat{u}(z)$ is anti-symmetric. But from (4.39), $\overline{b(z)}(i z+\beta)=-\overline{b(-z)}(-i z+$ $\beta$ ), so that in the case $\beta=q$, we see that $\bar{b}$ and $\hat{u}$ have the same symmetry. Thus the Bäcklund extension is not only the nonlinear analog of the method of images; it is the non-linearization of the method of images.

The situation is even more striking in "u-space". Indeed, from (4.5), $\tilde{u}=u-2 q \frac{\xi_{1} \overline{\xi_{2}}}{\left|\xi_{1}\right|^{2}+\left|\xi_{2}\right|^{2}}$, where $\xi=\left(\xi_{1}, \xi_{2}\right)^{T}$ solve

$$
\partial_{x} \xi=(i z \sigma+Q) \xi, \quad \xi(x=0)=e_{1} .
$$

Iterating (4.49) for $u$ small, we obtain $\xi(x)=\left(e^{-q x / 2},-\int_{0}^{x} e^{q(x-2 y) / 2} \bar{u}(y) \mathrm{d} y\right)^{T}+$ $O\left(u^{2}\right)$. Thus $\tilde{u}(x)=u(x)+2 q \int_{0}^{x} e^{q(x-2 y) / 2} \bar{u}(y) \mathrm{d} y+O\left(u^{2}\right)$. As $u(x)=$ $\tilde{u}(-x)$ for $x<0$, we see that $u^{e}(x)$ is precisely the same as $u_{e}$ in (1.30), up to terms of order $u^{2}$.

The following result is an immediate consequence of Theorem 5 and Proposition 4.28. Recall from (3.26) that $r \in H^{1,1}(\mathbb{R})$ if and only if $b \in H^{1,1}(\mathbb{R})$.

Corollary 4.32. Let $\left(r, Z_{+}, K_{+}\right) \in H^{1,1} \times \mathbb{C}_{+}^{n} \times(\mathbb{C} \backslash 0)^{n}$. Define $a(z)$ as in (3.24) and set $b(z)=a(z) \overline{r(z)}$. If $\left\{a(z), b(z),\left\{z_{k}\right\},\left\{\gamma_{k}\right\}, \beta\right\}$ satisfies (4.39), then $u=\mathcal{I}\left(r, Z_{+}, K_{+}\right) \in H^{1,1}(\mathbb{R})$ and is q-symmetric. 
Remark 4.33. Consider the 1-parameter family of 1-soliton solutions $e^{i \lambda^{2} t / 2} \eta_{\lambda}$ of NLS,

$$
\eta_{\lambda}(x)=\lambda \operatorname{sech}\left(\lambda x+\tanh ^{-1}(q / \lambda)\right), \quad|q|<\lambda .
$$

A direct calculation shows that the scattering data of $Q=\left(\begin{array}{cc}0 & \eta_{\lambda} \\ -\overline{\eta_{\lambda}} & 0\end{array}\right)$ is given by

$$
n=1, \quad z_{1}=i \lambda, \quad a(z)=\frac{z-i \lambda}{z+i \lambda}, \quad b(z) \equiv 0, \quad \gamma(i \lambda)=\sqrt{\frac{\lambda+q}{\lambda-q}},
$$

from which we immediately check by (4.39) that $\eta_{\lambda}$ is $q$-symmetric. Note that $\eta_{\lambda}$ coincides with the nonlinear ground state $v_{\lambda}$ for $x \geq 0$. In view of Remark 4.17, the Bäcklund extension of $\left.v_{\lambda}\right|_{\mathbb{R}^{+}}$is $\eta_{\lambda}$.

Theorem 6, Proposition 4.18, Proposition 4.25 and Proposition 4.28 imply the following important result.

Proposition 4.34. Let $u(t)$ solve $N L S$ on $\mathbb{R}$ with $u(t=0)=u_{0} \in$ $H^{1,1}(\mathbb{R})$. If $u_{0}$ is $q$-symmetric and generic, then $u(t)$ is $q$-symmetric for all $t \geq 0$. If $u(t)$ is a classical solution, then $\left.u(t)\right|_{\mathbb{R}^{+}}$and $\mathcal{R} \mathcal{B}_{q}^{+}\left(\left.u(t)\right|_{\mathbb{R}^{+}}\right)$ solve $H N L S_{q}^{ \pm}$, respectively.

From Proposition 4.7 the Bäcklund extension $u^{e}$ of $u \in H^{1,1}\left(\mathbb{R}^{+}\right)$ belongs to $H^{1,1}(\mathbb{R})$. In order to compute the scattering data for $u^{e}$, we need to know $u^{e} \in \mathcal{G}$. We have the following proposition.

Proposition 4.35 (Scattering data for the Bäcklund extension). Let $u(x) \in H^{1,1}\left(\mathbb{R}^{+}\right)$and let $g(x, z)$ be the (unique) solution of (3.5) for $x \geq 0, z \in \overline{\mathbb{C}^{+}}$given in (4.13) with $g(x, z) e^{-i x z / 2} \rightarrow e_{1}$ as $x \rightarrow \infty$. Denote $\left(\begin{array}{l}A(z) \\ B(z)\end{array}\right) \equiv g(0, z)$. Define

$$
\beta= \begin{cases}q, & \text { if } q>0, B(i q)=0 \quad \text { or } \quad q<0, A(-i q) \neq 0 \\ -q, & \text { if } q>0, B(i q) \neq 0 \quad \text { or } \quad q<0, A(-i q)=0\end{cases}
$$

and for $z \in \overline{\mathbb{C}^{+}}$, set

$$
a(z) \equiv \frac{1}{z-i \beta}((z-i q) A(z) \overline{A(-\bar{z})}-(z+i q) B(z) \overline{B(-\bar{z})}) .
$$

Suppose that $a(z)$ has only a finite number of zeros $z_{1}, \ldots, z_{n}, n \geq 0$, in $\overline{\mathbb{C}^{+}}$, all of which are simple and lie in $\mathbb{C}^{+}$. For $z \in \mathbb{R}$, set

$$
b(z) \equiv \frac{1}{z+i \beta}((z+i q) A(-z) B(z)+(z-i q) A(z) B(-z)),
$$


For $1 \leq k \leq n$, set

$$
\gamma\left(z_{k}\right) \equiv \begin{cases}\frac{z_{k}-i \beta}{z_{k}+i q} \frac{A\left(z_{k}\right)}{\overline{B\left(-\overline{z_{k}}\right)}}, & \text { if } A\left(z_{k}\right) \neq 0, \\ \frac{z_{k}-i \beta}{z_{k}-i q} \frac{B\left(z_{k}\right)}{\overline{A\left(-\overline{z_{k}}\right)}}, & \text { if } A\left(z_{k}\right)=0,\end{cases}
$$

Then, the Bäcklund extension $u^{e}$ of $u$ is generic and $\left\{a(z), b(z),\left\{z_{k}\right\}\right.$, $\left.\left\{\gamma_{k}\right\}\right\}$ is the scattering data $\mathcal{S}\left(u^{e}\right)$ for $u^{e}$. Moreover, $\mathcal{S}\left(u^{e}\right)$ satisfies the symmetries (4.39).

Proof. Let $u^{e}$ be the Bäcklund extension of $u$ and let $P^{e}(x)$ solve (4.1). As $u^{e}$ is $q$-symmetric, we have $P^{e}(x) \rightarrow-i \beta^{e} \sigma_{3}$ as $|x| \rightarrow \infty$, $\left(\beta^{e}\right)^{2}=q^{2}$. Let $\left(\psi^{e}\right)^{ \pm}$be the associated ZS-AKNS solutions. It is easy to check from the proof of Lemma 4.8 that in fact $\beta^{e}=\beta$. Clearly $\left(\psi^{e}\right)_{1}^{+}(x, z)=g(x, z), x \geq 0, z \in \overline{\mathbb{C}^{+}}$and hence $\left(\psi^{e}\right)_{1}^{+}(0, z)=g(0, z)=$ $(A(z), B(z))^{T}$. As $u^{e}$ is $q$-symmetric, we see from (4.34) that

$$
\left(\psi^{e}\right)_{1}^{-}(0, z)=\frac{1}{z+i \beta}\left(\begin{array}{c}
(z+i q) A(-z) \\
-(z-i q) B(-z)
\end{array}\right), \quad z \in \mathbb{R} .
$$

Thus, by (3.30), $b^{e}(z) \equiv b\left(z ; u^{e}\right)=\operatorname{det}\left(\left(\psi^{e}\right)_{1}^{-},\left(\psi^{e}\right)_{1}^{+}\right)(0, z)=b(z)$. On the other hand, it follows from (4.37) that

$$
\left(\psi^{e}\right)_{2}^{-}(0, z)=\frac{1}{z-i \beta}\left(\begin{array}{l}
(z+i q) \overline{B(-\bar{z})} \\
(z-i q) \overline{A(-\bar{z})}
\end{array}\right), \quad z \in \overline{\mathbb{C}^{+}} .
$$

So, the scattering function $a^{e}(z) \equiv a\left(z ; u^{e}\right)=\operatorname{det}\left(\left(\psi^{e}\right)_{1}^{+},\left(\psi^{e}\right)_{2}^{-}\right)(0, z)=$ $a(z)$. From this fact, we see that $a^{e}(z)$ has only a finite number of zeros $z_{1}, \ldots, z_{n}, n \geq 0$, in $\overline{\mathbb{C}^{+}}$, all of which are simple and lie in $\mathbb{C}^{+}$. Moreover, if $A\left(z_{k}\right) \neq 0$, then $\left(\left(\psi^{e}\right)_{1}^{+}\right)_{1}\left(0, z_{k}\right)=A\left(z_{k}\right) \neq 0$ and so $\left(\left(\psi^{e}\right)_{2}^{-}\right)_{1}\left(0, z_{k}\right) \neq$ 0 . Thus the norming constant $\gamma^{e}\left(z_{k}\right) \equiv \gamma\left(z_{k} ; u^{e}\right)=\frac{\left(\left(\psi^{e}\right)_{1}^{+}\right)_{1}\left(0, z_{k}\right)}{\left(\left(\psi^{e}\right)_{2}^{-}\right)_{1}\left(0, z_{k}\right)}=$ $\frac{z_{k}-i \beta}{z_{k}+i q} \frac{A\left(z_{k}\right)}{\overline{B\left(-\overline{z_{k}}\right)}}$. If $A\left(z_{k}\right)=0$, then $B\left(z_{k}\right) \neq 0$ and hence $\left(\left(\psi^{e}\right)_{1}^{+}\right)_{2}\left(0, z_{k}\right)=$ $B\left(z_{k}\right) \neq 0$. So, $\left(\left(\psi^{e}\right)_{2}^{-}\right)_{2}\left(0, z_{k}\right) \neq 0$ and hence $\gamma^{e}\left(z_{k}\right)=\frac{\left(\left(\psi^{e}\right)_{1}^{+}\right)_{2}\left(0, z_{k}\right)}{\left(\left(\psi^{e}\right)_{2}^{-}\right)_{2}\left(0, z_{k}\right)}=$

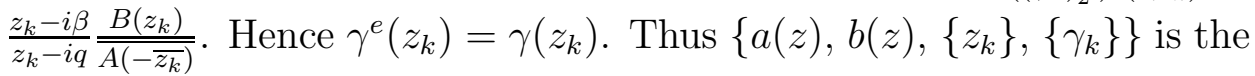
scattering data $\mathcal{S}\left(u^{e}\right)$ for $u^{e}$; the fact that $u^{e} \in \mathcal{G}$, follows directly from our assumption on $a(z)$.

Remark 4.36. The fact that $\left\{a(z), b(z),\left\{z_{k}\right\},\left\{\gamma_{k}\right\}\right\}$ is the scattering data $\mathcal{S}\left(u^{e}\right)$ for $u^{e}$, implies a variety of regularity properties for $a(z), b(z)$. In particular, it follows necessarily that if $\beta=|q|$, then the apparent singularity of $a(z)$ at $z=i \beta$ is removable: this can be seen directly as 
follows: $\beta=|q|$ if $q>0$ and $B(i q)=0$, or $q<0$ and $A(-i q)=0$. In the first case we have

$$
a(z)=A(z) \overline{A(-\bar{z})}-\frac{z+i q}{z-i q} B(z) \overline{B(-\bar{z})}
$$

and in the second case we have

$$
a(z)=\frac{z-i q}{z+i q} A(z) \overline{A(-\bar{z})}-B(z) \overline{B(-\bar{z})} .
$$

In both cases, the analyticity of $a(z)$ is clear. Furthermore, as $z \rightarrow i|q|$, $a(z) \rightarrow|A(i|q|)|^{2}$ or $-|B(i|q|)|^{2}$, respectively. As $A(z)$ and $B(z)$ cannot vanish simultaneously, we see that $a(i|q|) \neq 0$ in both cases. If $q>0$ and $B(i q) \neq 0$, then $\beta=-q$ and $a(i|q|)=a(i q)=-|B(i|q|)|^{2} \neq 0$ and if $q<0$ and $A(-i q) \neq 0$, then $\beta=q$ and $a(i|q|)=a(-i q)=$ $|A(i|q|)|^{2} \neq 0$. This shows that $a(z)$ is analytic in $\mathbb{C}^{+}$and non-zero at $z=i|q|$.

To see that the norming constant $\gamma\left(z_{k}\right)$ in (4.54) are well-defined and non-zero, suppose that $B\left(-\overline{z_{k}}\right) \neq 0$ : if $A\left(z_{k}\right)=0$, then as $a\left(z_{k}\right)=0$, we must have from (4.52) that $B\left(z_{k}\right)=0$, which contradicts the fact that $A(z)$ and $B(z)$ cannot vanish simultaneously. Thus $A\left(z_{k}\right) \neq 0$ and $\gamma\left(z_{k}\right)$ is well-defined and non-zero. If $B\left(-\overline{z_{k}}\right)=0$, then $A\left(-\overline{z_{k}}\right) \neq 0$. If $B\left(z_{k}\right)=0$, then again from (4.52) we see that $A\left(z_{k}\right)=0$, which is a contradiction. Thus we see that in all cases $\gamma\left(z_{k}\right)$ is well-defined and non-zero. Finally, the fact that $|a(z)|^{2}+|b(z)|^{2}=1, z \in \mathbb{R}$, in a direct calculation using (4.52) (4.53) together with $|A(z)|^{2}+|B(z)|^{2}=1$, $z \in \mathbb{R}$, from (3.20). The symmetry properties (4.39) can also be verified directly.

Remark 4.37. As noted in [Tar91], p.437, in the repulsive case $\kappa=$ -1 in (1.1), corresponding to the defocusing NLS equation, one can similarly construct the Bäcklund extension $u^{e}$ of $u$ as above. In this case, however, $a(z)$ may have a zero on the imaginary axis, so that the spectral data for $u^{e}$ is singular. This is the analog in the nonlinear situation that the method of images in the linear case breaks down if $q>0$, as described in Section 1 .

The set of functions $u$ on $\mathbb{R}^{+}$whose Bäcklund extensions $u^{e}$ are generic, is open and dense in $H^{1,1}\left(\mathbb{R}^{+}\right)$. We have the following result.

Proposition 4.38. Let $\mathcal{G}^{+}$be the set of functions $u \in H^{1,1}\left(\mathbb{R}^{+}\right)$such that the scattering function $a(z)$ of the Bäcklund extension of $u$ has only a finite number zeros in $\overline{\mathbb{C}^{+}}$, all of which are simple and lie in $\mathbb{C}^{+}$. Then, $\mathcal{G}^{+}$is open and dense in $H^{1,1}\left(\mathbb{R}^{+}\right)$. 
Proof. For $u \in \mathcal{G}^{+}$, consider a small disk $D=D(u, \epsilon)$ in $H^{1,1}\left(\mathbb{R}^{+}\right)$ centered at $u$ with radius $\epsilon>0$. For $v \in D$, let $g(0, z)=g(0, z ; v)$, $a(z)=a(z ; v)$ and $\beta=\beta(v)$ be defined as in Proposition 4.35. By Lemma 7.1 ,

$$
\sup _{v \in D} \sup _{z \in \overline{\mathbb{C}}^{+}}|g(0, z ; v)-g(0, z ; u)| \leq c \epsilon
$$

We have $|a(z ; u)| \geq \hat{c}>0$ for $z \in \mathbb{R}$. Define $a_{1}(z ; v) \equiv a(z ; v)(z-i \beta(v))$. As $|z-i \beta(v)|=|z-i \beta(u)|, z \in \mathbb{R}$, it follows from (4.55) that

$$
|a(z ; v)|=\left|\frac{a_{1}(z ; v)}{z-i \beta(u)}\right| \geq|a(z ; u)|-\left|\frac{a_{1}(z ; v)-a_{1}(z ; u)}{z-i \beta(u)}\right| \geq \hat{c}-c \epsilon>0,
$$

for sufficiently small $\epsilon>0$. On the other hand, since $a(z ; u) \rightarrow 1$ as $z \rightarrow \infty$, by (4.55) again, we have for some $R>0,|a(z ; v)| \geq c^{\#}>0$ for $|z| \geq R$, uniformly for $v \in D$. Now $a_{1}(z ; u)$ has only a finite number of zeros in $\mathbb{C}^{+}$, all of which are simple. This is clear for $z \neq i \beta$. However, if $\beta=|q|>0$, then as $a(i|q| ; u) \neq 0, a_{1}(z ; u)$ has only a simple zero at $z=i|q|=i \beta$. Again by Lemma 7.1 together with Rouché's theorem, we see that $a_{1}(z ; v)$ also has only a finite number of zeros in $\mathbb{C}^{+}$all of which are simple. This proves that $\mathcal{G}^{+}$is open.

We will show that $\mathcal{G}^{+}$is dense in $H^{1,1}\left(\mathbb{R}^{+}\right)$. Let $u \in H^{1,1}\left(\mathbb{R}^{+}\right)$. We can assume that $u$ has compact support, say $[0, L]$. Let $p(x, s)=$ $u(x)+s v(x)$ where $s \in \mathbb{R}$, and $v \in H^{1,1}\left(\mathbb{R}^{+}\right)$has compact support in $\left[L, L^{\prime}\right], L^{\prime}>L$, and will be determined later. Denote the associated ZS-AKNS solution normalized at $+\infty$ by $\psi^{+}(x, z ; p), x \geq 0$. Let $m^{+}(x, z ; p)=\psi^{+}(x, z ; p) e^{-i x z \sigma}=\left(m_{1}^{+}, m_{2}^{+}\right), m_{1}^{+}(0, z ; p)=\left(\begin{array}{c}A(z ; p) \\ B(z ; p)\end{array}\right)$, $m_{1}^{+}(0, z ; u)=\left(\begin{array}{c}A(z) \\ B(z)\end{array}\right)$ and $f(x, z)=\left.\frac{\partial m_{1}^{+}(x, z ; p)}{\partial s}\right|_{s=0}$. Note that since $p(x, s)$ has compact support, $m^{+}(x, z ; p)$ is analytic in $z$-plane. By differentiating the integral equation for $m_{1}^{+}$with respect to $s$ at $s=0$, we obtain

$$
\begin{aligned}
f(x, z)= & -\int_{x}^{\infty}\left(\begin{array}{cc}
1 & 0 \\
0 & e^{-i z(x-y)}
\end{array}\right)\left(\begin{array}{cc}
0 & v \\
-\bar{v} & 0
\end{array}\right) m_{1}^{+}(y, z ; u) \\
& -\int_{x}^{\infty}\left(\begin{array}{cc}
1 & 0 \\
0 & e^{-i z(x-y)}
\end{array}\right)\left(\begin{array}{cc}
0 & u \\
-\bar{u} & 0
\end{array}\right) f(y, z) \\
= & \mathrm{I}+\mathrm{II} .
\end{aligned}
$$

Since $m_{1}^{+}(x, z ; u)=e_{1}$ for $x \geq L$ and $v(x)=0$ for $0 \leq x<L$, we have

$$
\mathrm{I}=e^{-i x z}\left(\begin{array}{c}
0 \\
c(z)
\end{array}\right), \quad 0 \leq x \leq L
$$


where $c(z)=\int_{L}^{L^{\prime}} \overline{v(y)} e^{i z y}$. Therefore, for $0 \leq x<L$,

$$
e^{i x z} f(x, z)=\left(\begin{array}{c}
0 \\
c(z)
\end{array}\right)-\int_{x}^{\infty}\left(\begin{array}{cc}
e^{i z(x-y)} & 0 \\
0 & 1
\end{array}\right)\left(\begin{array}{cc}
0 & u \\
-\bar{u} & 0
\end{array}\right) e^{i y z} f(y, z) .
$$

Since $c(z) m_{2}^{+}(x, z ; u), z \in \mathbb{R}$, solves the same integral equation, it follows by uniqueness that

$$
e^{i x z} f(x, z)=c(z) m_{2}^{+}(x, z ; u) .
$$

for $0 \leq x<L$ and $z \in \mathbb{R}$. But then by analytic continuation, (4.56) remains true for all $z \in \mathbb{C}, 0 \leq x<L$. Thus,

$$
\left.\frac{\partial}{\partial s}\right|_{s=0}\left(\begin{array}{l}
A(z ; p) \\
B(z ; p)
\end{array}\right)=f(0, z)=c(z) m_{2}^{+}(0, z ; u)=c(z)(\overline{-\overline{B(\bar{z})}}) .
$$

Let $a_{1}(z, s) \equiv a_{1}(z ; p)=a(z ; p)(z-i \beta(p))$. Then,

$$
\frac{\partial a_{1}}{\partial s}(z, 0)=c(z) \overline{\mu(-\bar{z})}-\overline{c(-\bar{z})} \mu(z)
$$

where

$$
\mu(z)=(z-i q) A(z) B(-z)+(z+i q) A(-z) B(z) .
$$

Now we prove that, for suitable choice of $v$,

$$
\frac{\partial a_{1}}{\partial s}(\xi, 0) \neq 0
$$

for any zero $\xi$ of $a(z)=a(z ; u)$. We first show that $\mu(\xi)$ and $\mu(-\bar{\xi})$ do not vanish simultaneously. Recall that

$$
\operatorname{det} m^{+}(x, z) \equiv 1 \Rightarrow A(z) \overline{A(\bar{z})}+B(z) \overline{B(\bar{z})}=1, z \in \mathbb{C} .
$$

Suppose that $a(\xi)=0, \mu(\xi)=0$ and $\mu(-\bar{\xi})=0$ for some $\xi \in \mathbb{C}^{+}$. Note that $\xi \neq i|q|$. If $B(\xi)=0, A(\xi) \neq 0$. But then as $a(\xi)=0, A(-\bar{\xi})=0$ by (4.52). But then $B(-\bar{\xi}) \neq 0$ and it follows from $\mu(-\bar{\xi})=0$ that $A(\bar{\xi})=0$. This contradicts (4.60) and hence $B(\xi) \neq 0$. On the other hand, if $A(-\bar{\xi})=0$, then as $a(\xi)=0$ and $B(\xi) \neq 0$, we must have $B(-\bar{\xi})=0$, which is a contradiction. Hence $A(-\bar{\xi}) \neq 0$. Now as $a(\xi)=0,(\xi-i q) A(\xi)=(\xi+i q) \frac{\overline{B(-\bar{\xi})}}{\overline{A(-\bar{\xi})}} B(\xi)$ and hence by (4.60) again,

$$
\begin{aligned}
\mu(\xi) & =(\xi+i q)\left(\frac{\overline{B(-\bar{\xi})}}{\overline{A(-\bar{\xi})}} B(\xi) B(-\xi)+A(-\xi) B(\xi)\right) \\
& =(\xi+i q) \frac{B(\xi)}{\overline{A(-\bar{\xi})}} \neq 0,
\end{aligned}
$$


which is a contradiction. Now, as $a(z ; u)$ is analytic across $\mathbb{R}$ and $a(z ; u) \rightarrow 1$ as $z \rightarrow \infty, z \in \overline{\mathbb{C}^{+}}, a(z ; u)$ has only a finite number of zeros in $\overline{\mathbb{C}^{+}}$. As $c(z)=\int_{L}^{L^{\prime}} \overline{v(y)} e^{i z y}$ and $\overline{c(-\bar{z})}=\int_{L}^{L^{\prime}} v(y) e^{i z y}$, using the above results, we can find a function $v$ such that (4.59) is satisfied for all the zeros of $a(z ; u)$. For example, a simple way to do this is to consider $v=e^{i \theta} \delta_{L}(x)$, where $\delta_{L}(x)$ is a delta function at $x=L$, and then choose $\theta \in \mathbb{R}$ appropriately; smoothing out $v$ then produces the desired $v$. But then, by Proposition 4.39 below, all the zeros of $a_{1}(z ; p)$ are simple for sufficiently small $s \neq 0$ and hence so are those of $a(z ; p)$. In other words, there exists $p(x)$ which has compact support such that $a(z ; p)$ has only a finite number of zeros in $\overline{\mathbb{C}^{+}}$, all of which are simple. We now show that any real zeros of $a(z ; p)$, if there are any, can be moved off the real axis by perturbing $p(x) \rightarrow p_{\hat{s}}(x)=p(x)+\hat{s} \hat{v}(x)$, where $\hat{v}(x)$ again has compact support, and $\hat{s} \neq 0$ is arbitrarily small. By standard analysis, if $\xi$ is a simple zero of $a\left(z ; p_{\hat{s}=0}\right)$, then for $\hat{s}$ small, $a\left(z ; p_{\hat{s}}\right)$ has simple zero $\xi(\hat{s})$ near $\xi=\xi(0), a\left(\xi(\hat{s}) ; p_{\hat{s}}\right)=0$ and $\xi(\hat{s})$ is differentiable. As $a_{1}\left(\xi(\hat{s}) ; p_{\hat{s}}\right)=0$, we have

$$
0=\xi^{\prime}(0) a_{1}^{\prime}(\xi(0) ; p)+\frac{\partial a_{1}}{\partial \hat{s}}(\xi(0) ; p)
$$

Here (4.58) is given with $u$ replaced by $p$. If $a(z ; p)$ has real zeros, as $a_{1}^{\prime}(\xi(0) ; p) \neq 0$, it is easy to see that we can choose $\hat{v}$ such that (4.59) is satisfied and $\xi^{\prime}(0)$ is not real for all (the finite number of) real zeros $\xi(0)$ of $a(z ; p)$. Thus, for sufficiently small $\hat{s} \neq 0$, all the zeros of $a\left(z ; p_{\hat{s}}\right)$ in $\overline{\mathbb{C}^{+}}$are simple and non-real. This completes the proof that $\mathcal{G}^{+}$is open and dense in $H^{1,1}\left(\mathbb{R}^{+}\right)$.

Proposition 4.39 ([BDT88], p.72). Suppose $U$ is a neighborhood of the origin in $\mathbb{C} \times \mathbb{R}$ and suppose $h: U \rightarrow \mathbb{C}$ is smooth and holomorphic with respect to the first variable. Suppose

$$
h(0,0)=0, \quad \frac{\partial h}{\partial s}(0,0) \neq 0, \quad h=h(z, s) .
$$

Then, for small $s \neq 0$, the zeros of $h(\cdot, s)$ near $z=0$ are simple.

Remark 4.40. It is of interest to see whether the scattering function $a(z)$ for the Bäcklund extension of a function $u \in H^{1,1}\left(\mathbb{R}^{+}\right)$, can indeed have zeros on $\mathbb{R}$. Fix any integer $n \geq 0$ and let $\xi=\sqrt{2}\left(n+\frac{1}{2}\right) \pi>0$ and $q=\xi \cot \xi$ ia finite and non-zero. Define $u(x)=\frac{\xi}{2} \chi_{[1,2]}, x \geq 0$ where $\chi$ is a characteristic function. Then, a direct calculation shows that for 
$z \in \mathbb{R}$

$$
\left\{\begin{array}{l}
A(z)=e^{\frac{i z}{2}}\left\{\cos W-\frac{i z}{2 W} \sin W\right\}, \\
B(z)=\frac{\xi e^{\frac{3 i z}{2}}}{2 W} \sin W
\end{array}\right.
$$

where $W=W(z) \equiv \frac{1}{2} \sqrt{\xi^{2}+z^{2}}$. Since $\overline{A(-z)}=A(z)$ and $\overline{B(-z)}=$ $B(z)$, by (4.52),

$$
a(z)=\frac{z-i q}{z-i \beta}\left\{(A(z))^{2}-\frac{z+i q}{z-i q}(B(z))^{2}\right\}, \quad z \in \mathbb{R} .
$$

and it is easy to check that $a(z)$ vanishes at $\pm \xi$.

Before we present the proof of Theorem 7, we need the following lemma of approximation.

Lemma 4.41. Suppose $u \in H^{1,1}(\mathbb{R})$. Then there exists a sequence of smooth functions $\left\{u_{n}\right\}$ with compact support in $[0, \infty)$ such that

(i) $u_{n} \rightarrow u$ in $H^{1,1}\left(\mathbb{R}^{+}\right)$,

(ii) $u_{n}^{\prime}(0)+q u_{n}(0)=0$, and

(iii) the Bäcklund extension $u_{n}^{e}$ of $u_{n}$ is generic.

Proof. As the set $\mathcal{G}^{+}$in Proposition 4.38 is open and dense, it is enough to show that there exists a sequence of smooth functions $\left\{u_{n}\right\}$ with compact support in $[0, \infty)$ such that (i) and (ii) hold. By standard estimates, there exist smooth functions $u_{n}^{(1)}$ with compact support in $\left[\frac{1}{2}, \infty\right)$ such that $u_{n}^{(1)} \rightarrow u$ in $H^{1,1}\left[\frac{1}{2}, \infty\right)$. Now let $\left\{f_{n}\right\}$ be a sequence of functions in $C_{0}^{\infty}(0,1)$ such that $f_{n} \rightarrow f \equiv u^{\prime}+q u$ in $L^{2}[0,1]$. Set $u_{n}^{(2)} \equiv e^{-q x} u(0)+\int_{0}^{x} e^{-q(x-s)} f_{n}(s) \mathrm{d} s, x \in[0,1]$. Then $u_{n}^{(2)}(x)$ is smooth and $\left(u_{n}^{(2)}\right)^{\prime}(x)+q u_{n}^{(2)}(x)=f_{n}(x)$, so that $\left(u_{n}^{(2)}\right)^{\prime}(0)+q u_{n}^{(2)}(0)=f_{n}(0)=$ 0 . Also $u_{n}^{(2)}$ converges in $L^{2}[0,1], u_{n}^{(2)} \rightarrow u^{(2)}$, and we have $u^{(2)}(x)=$ $e^{-q x} u(0)+\int_{0}^{x} e^{-q(x-s)}\left(u^{\prime}(s)+q u(s)\right) \mathrm{d} s=u(x)$. But then $\left(u_{n}^{(2)}\right)^{\prime}=$ $f_{n}-q u_{n}^{(2)} \rightarrow\left(u^{\prime}+q u\right)-q u=u^{\prime}$, also in $L^{2}[0,1]$. Let $\chi \in C_{0}^{\infty}\left(\mathbb{R}^{+}\right)$such that $0 \leq \chi(x) \leq 1, \chi(x)=0$ for $0 \leq x \leq \frac{1}{2}$, and $\chi(x)=1$ for $x \geq 1$. Let $u_{n}=u_{n}^{(1)} \chi+u_{n}^{(2)}(1-\chi)$. Then clearly $u_{n} \rightarrow u$ in $H^{1,1}\left(\mathbb{R}^{+}\right)$and $u_{n}^{\prime}(0)+q u_{n}(0)=0$.

Proof of Theorem 7. Suppose that $q>0$. The case $q<0$ is similar. Let $g(x, z)=\left(g_{1}, g_{2}\right)^{T}$ be as in the proof of Proposition 4.7. If $g_{2}\left(0, i q ; u_{0}^{+}\right) \neq 0$, then by Lemma 4.41, we can choose a sequence $\left\{u_{0}^{(n)}\right\}$ of $C^{\infty}$-functions with compact support in $[0, \infty)$ such that

(1) $u_{0}^{(n)} \rightarrow u_{0}$ as $n \rightarrow \infty$ in $H^{1,1}\left(\mathbb{R}^{+}\right)$, 
(2) $\left(u_{0}^{(n)}\right)^{\prime}(0+)+q u_{0}^{(n)}(0)=0$, and

(3) the Bäcklund extension $\left(u_{0}^{(n)}\right)^{e}$ of $u_{0}^{(n)}$ is generic.

As $u_{0}^{(n)}$ has compact support, it is easy to verify from (4.5) that $\mathcal{R B}_{q}^{+} u_{0}$ is also smooth and decays at least exponentially. It follows by Proposition 4.26 that $\left(u_{0}^{(n)}\right)^{e} \in H^{3,3}(\mathbb{R})$ and hence it follows by Proposition 2.1 that NLS has a solution $u_{n}^{e}(t)=u_{n}^{e}(x, t)$ in $H^{3,3}(\mathbb{R})$ with $u_{n}^{e}(t=0)=\left(u_{0}^{(n)}\right)^{e}$. In particular, $u_{n}^{e}(t)$ is a classical solution of NLS. By Proposition 4.34 together with the properties of $u_{0}^{(n)}$ above, we see that $\left.u_{n}^{e}(t)\right|_{\mathbb{R}^{+}}$solves $\mathrm{HNLS}_{q}^{+}$. On the other hand, by Lemma 4.24 below, we see that $\left(u_{0}^{(n)}\right)^{e} \rightarrow u_{0}^{e}$ in $H^{1,1}(\mathbb{R})$ and hence $u_{n}^{e}(t) \rightarrow u^{e}(t)$ in $H^{1,1}(\mathbb{R})$ as $n \rightarrow \infty$, uniformly for $t$ in any interval $[0, T], 0<T<\infty$. In particular, $\left.\left.u_{n}^{e}(t)\right|_{\mathbb{R}^{+}} \rightarrow u^{e}(t)\right|_{\mathbb{R}^{+}}$as $n \rightarrow \infty$. Hence, it follows that $\left.u^{e}(t)\right|_{\mathbb{R}^{+}}$also solves $\mathrm{HNLS}_{q}^{+}$.

If $g_{2}\left(0, i q ; u_{0}^{+}\right)=0$, then define $G(x, z)=\left(G_{1}, G_{2}\right)^{T}$ as in the proof of Proposition 4.12. We obtain $G_{2}\left(0, i q ; \mathcal{R B}_{q}^{+} u_{0}^{+}\right) \neq 0$ and hence we are in the similar situation to the case $g_{2}\left(0, i q ; u_{0}^{+}\right) \neq 0$, but now on $\mathbb{R}^{-}$ with $v_{0} \equiv \mathcal{R} \mathcal{B}_{q}^{+} u_{0}^{+}$. We choose a sequence $\left\{v_{0}^{(n)}\right\}$ which converges to $v_{0}$ in $H^{1,1}\left(\mathbb{R}^{-}\right)$and gives rise to a classical solution $v_{n}(t)$ with $v_{n}(t=0)=$ $\left(v_{0}^{(n)}\right)^{e}$, where $\left(v_{0}^{(n)}\right)^{e}$ is the Bäcklund extension of $v_{0}^{(n)} \in H^{1,1}\left(\mathbb{R}^{-}\right)$. Let $v_{0}^{e}$ be the Bäcklund extension of $v_{0} \in H^{1,1}\left(\mathbb{R}^{-}\right)$. By Proposition 4.18 below, we see that $v_{0}^{e}=u_{0}^{e}$ and hence $\left(v_{0}^{(n)}\right)^{e} \rightarrow u_{0}^{e}$. Thus, $\left.u^{e}(t)\right|_{\mathbb{R}^{+}}=$ $\left.\lim _{n \rightarrow \infty} v_{n}(t)\right|_{\mathbb{R}^{+}}$solves $\mathrm{HNLS}_{q}^{+}$.

Now it follows immediately that $u(x, t) \equiv u^{e}(|x|, t)$ solves the IVP (1.4) with $u(x, 0)=u_{0}(x)$ in the sense of (1.8).

Remark 4.42. It follows from the proof of Theorem 7 , that if $u(t) \in$ $H^{1,1}\left(\mathbb{R}^{+}\right), u(t=0)=u_{0}$, is a solution of $\operatorname{HNLS}_{q}^{+}$, then there exists a sequence of solutions $u_{n}(t) \in H^{3,3}\left(\mathbb{R}^{+}\right)$of $\mathrm{HNLS}_{q}^{+}$, such that for $t \geq 0$, $\left\|u_{n}(t)-u(t)\right\|_{H^{1,1}(\mathbb{R})} \rightarrow 0$ as $n \rightarrow \infty$. In particular, any solution to $\mathrm{HNLS}_{q}^{+}$in $H^{1,1}\left(\mathbb{R}^{+}\right)$can be approximated by a classical solution to $\mathrm{HNLS}_{q}^{+}$. With a little more work, one can show that the $u_{n}$ 's can be chosen in $H^{k, k}\left(\mathbb{R}^{+}\right)$for any $k \geq 3$.

Remark 4.43. It also follows from the proof of Theorem 7 , that if $u_{0} \in$ $H^{1,1}(\mathbb{R})$ and $u^{e}(t)$ is the solution of NLS on $\mathbb{R}$ with $u^{e}(t=0)=u_{0}^{e}$, the Bäcklund extension of $u_{0}$, then $u^{e}(t)$ is $q$-symmetric (cf. Proposition 4.34).

Proposition 4.44. Let $k \geq 2$ and suppose $u_{0} \in H^{k, k}\left(\mathbb{R}^{+}\right)$. In addition, suppose that the Bäcklund extension $u_{0}^{e}$ of $u_{0}$ is in $H^{k, k}(\mathbb{R})$. Then 
$H N L S_{q}^{+}$has a unique solution $u(t)$ in $H^{k, k}\left(\mathbb{R}^{+}\right)$with $u(t=0)=u_{0}$ and satisfies the boundary condition $u_{x}(0+, t)+q u(0+, t)=0, t \geq 0$.

Proof. As $u_{0}^{e} \in H^{k, k}(\mathbb{R})$, NLS has a solution $u^{e}(t)$ in $H^{k, k}(\mathbb{R})$ with $u^{e}(t=0)=u_{0}^{e}$. But then, by Theorem 7, as $H^{k, k} \subset H^{1,1},\left.u^{e}(t)\right|_{\mathbb{R}^{+}}$satisfies (1.9), and hence $\left.u^{e}(t)\right|_{\mathbb{R}^{+}}$is a solution of $\operatorname{HNLS}_{q}^{+}$in $H^{k, k}\left(\mathbb{R}^{+}\right)$. Furthermore, as $H^{k, k}\left(\mathbb{R}^{+}\right) \subset C^{1}\left(\mathbb{R}^{+}\right)$, for $k \geq 2$, and $u^{e}(t)$ is $q$-symmetric (see Remark 4.43), the boundary condition at $x=0$ follows from Proposition 4.25.

Remark 4.45. Let $k \geq 2$. As $u_{0}^{e} \in H^{k, k}(\mathbb{R})$, the boundary condition $u_{0}^{\prime}(0+)+q u_{0}(0+)=0$ is automatic for $u_{0}$ (see Proposition 4.25).

The condition above on $u_{0}^{e}$ is rather implicit. Of course, the condition can be rephrased in terms of requirement on the derivatives of $u_{0}$ at $x=0+$. For $k=2$ or 3 , the conditions are particularly simple.

Proposition 4.46. Suppose $u_{0} \in H^{k, k}\left(\mathbb{R}^{+}\right), k=2$ or 3 , and in addition, suppose that $u_{0}^{\prime}(0+)+q u_{0}(0+)=0$. Then $H N L S_{q}^{+}$has a unique solution $u(t)$ in $H^{k, k}\left(\mathbb{R}^{+}\right), k=2$ or 3 , with $u(t=0)=u_{0}$ and satisfying the boundary condition $u_{x}(0+, t)+q u(0+, t)=0, t \geq 0$.

Proof. It is enough to show that $u_{0}^{e}$ is in $H^{k, k}, k=2,3$. Let $w=\mathcal{R B}_{q}^{+} u_{0}$. We must show that for $k=2$,

(i) $w(0-)=u_{0}(0+)$,

(ii) $w^{\prime}(0-)=u_{0}^{\prime}(0+)$,

and in addition, if $k=3$,

(iii) $w^{\prime \prime}(0-)=u_{0}^{\prime \prime}(0+)$.

As $H^{2,2} \subset C^{1}$, conditions (i)(ii) follow from (the proof of) Proposition 4.25. On the other hand, as $H^{3,3} \subset C^{2}$, condition (iii) follows from Proposition 4.26. The proof that the Bäcklund extension $u_{0}^{e}$ of $u_{0} \in$ $H^{k, k}\left(\mathbb{R}^{+}\right)$belongs to $H^{k, k}(\mathbb{R}), k=2$ or 3 , follows in a similar way to the proof that $u_{0}^{e}$ of $u_{0} \in H^{1,1}\left(\mathbb{R}^{+}\right)$lies in $H^{1,1}(\mathbb{R})$ (cf. Proposition 4.7).

Remark 4.47. For $k=3$, the solution $u(t)$ in $H^{3,3}$ is a classical solution.

Remark 4.48. By Proposition 4.46, solutions $u(t)$ of $\mathrm{HNLS}_{q}^{+}$in $H^{2,2}$ satisfy the boundary condition $u_{x}(0+, t)+q u(0+, t)=0, t \geq 0$. As $H^{2,2} \subset\left\{u \in H^{1,1}\left(\mathbb{R}^{+}\right): u^{\prime} \in L^{1}\left(\mathbb{R}^{+}\right)\right\}$, we see that this result is consistent with Step 2 above for $t>0$.

\section{Long-Time Asymptotics}

By the results of the previous section, in order to determine the longtime behavior of the solution $u(x, t)$ of the IVP (1.4), we need to analyze 
the solution $u^{e}(x, t)$ to the equation (3.1) with initial data given by the Bäcklund extension $u_{0}^{e}$ of $\left.u_{0}\right|_{\mathbb{R}^{+}}$. We consider only the case when $u_{0}^{e}$ is generic so that the associated RHP (3.31) has a finite number of simple poles in $\mathbb{C} \backslash \mathbb{R}$ (cf. Remark 3.4). Rather than analyzing the asymptotic behavior of the RHP directly, it is convenient to remove the poles using Darboux transformations (see the Appendix for details). Once the long time behavior of the RHP without poles is determined, we then add the poles back in, again using Darboux transformations. This procedure of removing the poles is not necessary: we can certainly apply the DeiftZhou steepest-descent method directly to RHP's with poles. However, in situations where there are only one or two pairs of poles, which is of primary interest here, it is more convenient to proceed by first stripping out the poles.

In order to explain the above procedure in more detail, suppose that the scattering data of $u^{e}(x, t=0)=u_{0}^{e}(x)$ is given by the reflection coefficient function $r(z)=\overline{b(z)} / \overline{a(z)}$ with $\|r\|_{H^{1,1}(\mathbb{R})}<\infty$, and one pair of simple eigenvalues at $z=z_{1} \equiv i \mu_{1} \in i \mathbb{R}^{+}$with the norming constant $\gamma_{1}=\gamma\left(z_{1}\right) \neq 0$, and at $\overline{z_{1}}$ with corresponding norming constant $-\overline{\gamma_{1}}$. Here, $a(z), b(z)$ and $\gamma_{1}$ are constructed from $u_{0} \in H^{1,1}\left(\mathbb{R}^{+}\right)$as in Proposition 4.35. Note that the norming constant $\gamma_{1}$ is of the form $\gamma_{1}=e^{-i \rho} \sqrt{\frac{\mu_{1}+q}{\mu_{1}-q}}$ for some $\rho \in \mathbb{R}$. Indeed, by Proposition 4.28,

$$
\gamma\left(z_{1}\right) \overline{\gamma\left(-\overline{z_{1}}\right)}=\frac{z_{1}-i \beta}{z_{1}+i \beta}, \quad \beta=-q \Rightarrow\left|\gamma\left(z_{1}\right)\right|=\sqrt{\frac{\mu_{1}+q}{\mu_{1}-q}} .
$$

The evolution of the scattering data is given as follows by Theorem 6 .

$$
z_{1}(t)=i \mu_{1}, \quad \gamma_{1}(t)=\gamma_{1} e^{i z_{1}^{2} t / 2}, \quad r(z, t)=r(z) e^{-i z^{2} t / 2} \text { for } z \in \mathbb{R}
$$

Define

$$
r_{f}(z) \equiv r(z) \frac{z-\overline{z_{1}}}{z-z_{1}} .
$$

By the Appendix, $r_{f}$ is the pole-free reflection coefficient that one obtains after removing the poles at $z=z_{1}, \overline{z_{1}}$ by using a Darboux transformation. For each fixed $x \in \mathbb{R}$ and $t>0$, let the $2 \times 2$ matrix function $m_{f}(x, t, z)$ solve the normalized $\operatorname{RHP}\left(\mathbb{R}, v_{f}\right): m_{f}(x, t, z)$ is analytic in $\mathbb{C} \backslash \mathbb{R}$ and the jump matrix is given by

$v_{f}(z)=v_{f}(x, t, z) \equiv\left(\begin{array}{cc}1+\left|r_{f}(z)\right|^{2} & r_{f}(z) e^{i \theta} \\ r_{f}(z) e^{-i \theta} & 1\end{array}\right), \theta=\theta(x, t, z) \equiv x z-\frac{t z^{2}}{2}$.

Let $u_{f}(x, t)$ be the associated potential function with $Q_{f}=\left(\begin{array}{cc}0 & u_{f} \\ -\overline{u_{f}} & 0\end{array}\right)$. If we apply the Darboux transformation to add in poles at $z_{1}=i \mu_{1}$, 
$\overline{z_{1}}=-i \mu_{1}$ with the norming constant $\gamma_{1}(t)=\gamma_{1} e^{i z_{1}^{2} t / 2}$, we obtain the solution $m(x, t, z)$ to the RHP corresponding to (5.2). In particular, we have from (9.3) and (9.5),

$$
u^{e}(x, t)=u_{f}(x, t)+i\left(z_{1}-\overline{z_{1}}\right) \mathcal{F}(\mathfrak{b})
$$

where

$$
\mathfrak{b}=m_{f}\left(z_{1}\right) e^{i x z_{1} \sigma}\left(\begin{array}{c}
1 \\
\frac{-c_{1}(t)}{z_{1}-\overline{z_{1}}}
\end{array}\right), \quad c_{1}(t)=\frac{\gamma_{1}(t)}{a^{\prime}\left(z_{1}\right)}
$$

The pole-free solution $u_{f}(x, t)$ can be read off from the residue of $m_{f}(x, t, z)$, i.e.,

$$
u_{f}(x, t)=-i\left(\left(m_{f}\right)_{1}(x, t)\right)_{12}, \quad\left(m_{f}\right)_{1}(x, t) \equiv \lim _{z \rightarrow \infty} z\left(m_{f}(x, t, z)-I\right) .
$$

The goal is to obtain an explicit formula for the asymptotic of $m_{f}(x, t, z)$ as $t \rightarrow \infty$; the behavior of $u^{e}(x, t)$ as $t \rightarrow \infty$ can then be read off from (5.5) and (5.6). The case where there are $n \geq 2$ simple eigenvalues, is treated similarly. Here we use

$$
r_{f}(z)=r(z) \prod_{k=1}^{n} \frac{z-\overline{z_{k}}}{z-z_{k}} .
$$

We now apply the steepest-descent method introduced by Deift and Zhou in DZ93 to the focusing NLS at hand (see also [DIZ93]). We will follow DZ03 in which the authors analyze the long-time behavior of the defocusing NLS equation, making suitable modification for the focusing case. Along the way we will state many technical results (e.g. Proposition 5.1, etc.) without proof: the proofs are very similar to analogous results in [DZ03] and the details are left to the reader.

In view of the preceding discussion we must consider normalized RHP's $(\Sigma=\mathbb{R}, v)$ on $\mathbb{R}$ with jump matrices of the form,

$$
v(z)=v(x, t, z)=\left(\begin{array}{cc}
1+|r(z)|^{2} & r(z) e^{i \theta} \\
r(z) e^{-i \theta} & 1
\end{array}\right), \theta=x z-\frac{t z^{2}}{2},
$$

where $r \in H^{1,1}(\mathbb{R})$. We are interested in the behavior of the solution $m=m(x, t, z)$ of $(\mathbb{R}, v)$ as $t \rightarrow \infty$.

Solutions $m=m(x, t, z)$ of the RHP as given in (3.31) are classical in the sense that $m$ is continuous up to the boundary in $\mathbb{C}^{+}$and $\mathbb{C}^{-}$. Such solutions $m$ exist because the reflection coefficient $r \in H^{1}(\mathbb{R})$. For a general RHP on an oriented contour $\Sigma \subset \mathbb{C}$ with jump matrix $v \in L^{\infty}(\Sigma)$, we must define the RHP in a weaker sense (see [LS87], CG81, and also DZ03]), as follows. 
Let $C_{\Sigma}$ denote the Cauchy operator

$$
\left(C_{\Sigma} h\right)(z)=\int_{\Sigma} \frac{h(s)}{s-z} \frac{\mathrm{d} s}{2 \pi i}, \quad z \in \mathbb{C} \backslash \Sigma,
$$

with associated boundary operators

$$
\left(C_{\Sigma}^{ \pm} h\right)(z)=\lim _{z^{\prime} \rightarrow z^{ \pm}}\left(C_{\Sigma} h\right)\left(z^{\prime}\right) .
$$

Here $z^{ \pm}$refer to the orientation of $\Sigma$ at the point $z \in \Sigma$ : by convention if one traverses the contour in the direction of the orientation, the $( \pm)$ sides lie to the left (right) respectively. For example, if $\Sigma=\mathbb{R}$ with orientation from $-\infty$ to $\infty$, then $z^{ \pm}$refer to limits as $z^{\prime} \rightarrow z \in \mathbb{R}$ from $\mathbb{C}^{+} / \mathbb{C}^{-}$respectively. If $\Sigma$ is sufficiently regular - Lipschitz would do the operators $C_{\Sigma}^{ \pm}$are bounded in $L^{p}(\Sigma), 1<p<\infty$ (see e.g. [BK97]), and the limits in (5.10) exist pointwise almost everywhere in $\Sigma$. We always have

$$
C_{\Sigma}^{+}-C_{\Sigma}^{-}=\mathrm{id}
$$

Given $\Sigma$ and a matrix $v$, we say that a pair $h_{ \pm}$of $L^{p}(\Sigma)$ functions lies in $\partial C\left(L^{p}\right)$ if $h_{ \pm}=C_{\Sigma}^{ \pm} g$ for some (unique) $g \in L^{p}(\Sigma)$. We say $m_{ \pm}$solves the normalized $\operatorname{RHP}(\Sigma, v)_{L^{2}}$, if

- $m_{ \pm} \in I+\partial C\left(L^{2}\right)$,

- $m_{+}(z)=m_{-}(z) v(z), z \in \Sigma$.

Note that if $m_{ \pm}$solves the normalized RHP $(\Sigma, v)$, then $m_{ \pm}=I+C_{\Sigma}^{ \pm} g$ for some $g \in L^{2}(\Sigma)$. Hence $m(z)=I+\left(C_{\Sigma} g\right)(z)=I+\int_{\Sigma} \frac{g(s)}{s-z} \frac{\mathrm{d} s}{2 \pi i}$, $z \in \mathbb{C} \backslash \Sigma$, is analytic in $\mathbb{C} \backslash \Sigma$, and clearly solves the $\operatorname{RHP}(\Sigma, v)$ a.e. on $\Sigma$. Although the original $\operatorname{RHP}(\mathbb{R}, v)$ has a classical solution, in the process of solving for $m(x, t, z)$ as $t \rightarrow \infty$, we will encounter RHP's which only have solutions in the generalized sense (see e.g. (5.12) below, etc.). It is easy to see that the classical solution $m(x, t, z)$ of $(\Sigma, v)$ is a also a solution in the generalized sense.

The first step in the steepest descent method is to separate $e^{i \theta}$ and $e^{-i \theta}$ in the jump matrix $v(x, t, z)$ in such a way as to respect the signature table for $\operatorname{Re}(i \theta)$. We have $\operatorname{Re}(i \theta)=\operatorname{Re}\left[i t\left(z_{0}^{2}-\left(z-z_{0}\right)^{2}\right) / 2\right]=$ $t \operatorname{Im}\left(z-z_{0}\right)^{2} / 2$ where $z_{0}=x / t$ is the stationary phase point for $\theta$. Let $\delta_{ \pm}$be the solution of the scalar, normalized RHP $\left(\mathbb{R}_{-}+z_{0}, 1+|r|^{2}\right)$,

$$
\left\{\begin{array}{l}
\delta_{+}=\delta_{-}\left(1+|r|^{2}\right), \quad z \in \mathbb{R}_{-}+z_{0}, \\
\delta_{ \pm}-1 \in \partial C\left(L^{2}\right),
\end{array}\right.
$$

where the contour $\mathbb{R}_{-}+z_{0}$ is oriented from $-\infty$ to $z_{0}$. 
Proposition 5.1 (Properties of $\delta$ ). Suppose $r \in L^{\infty}(\mathbb{R}) \cap L^{2}(\mathbb{R})$ and $\|r\|_{L^{\infty}} \leq \rho<\infty$. The extension $\delta$ of $\delta_{ \pm}$off $\mathbb{R}_{-}+z_{0}$ is given by the formula

$$
\delta(z)=\exp \left[\frac{1}{2 \pi i} \int_{-\infty}^{z_{0}} \frac{\log \left(1+|r(s)|^{2}\right)}{s-z} d s\right], \quad z \in \mathbb{C} \backslash\left(\mathbb{R}_{-}+z_{0}\right) .
$$

and satisfies for $z \in \mathbb{C} \backslash\left(\mathbb{R}_{-}+z_{0}\right)$,

$$
\begin{gathered}
\delta(z) \overline{\delta(\bar{z})}=1, \\
\left(1+\rho^{2}\right)^{-\frac{1}{2}} \leq|\delta(z)|,\left|\delta^{-1}(z)\right| \leq\left(1+\rho^{2}\right)^{\frac{1}{2}}
\end{gathered}
$$

and

$$
\left|\delta^{ \pm 1}(z)\right| \leq 1 \quad \text { for } \quad \pm \operatorname{Im} z>0 .
$$

For real $z$,

$$
\begin{gathered}
\left|\delta_{+}(z) \delta_{-}(z)\right|=1 \quad \text { and, in particular, } \quad|\delta(z)|=1 \quad \text { for } \quad z>z_{0}, \\
\left|\delta_{+}(z)\right|=\left|\delta_{-}^{-1}(z)\right|=\left(1+|r(z)|^{2}\right)^{\frac{1}{2}}, \quad z<z_{0},
\end{gathered}
$$

and

$$
\Delta \equiv \delta_{+} \delta_{-}=\exp \left[\frac{1}{i \pi} P . V . \int_{-\infty}^{z_{0}} \frac{\log \left(1+|r(s)|^{2}\right)}{s-z} d s\right],
$$

where P.V. denotes the principal value and $|\Delta|=\left|\delta_{+} \delta_{-}\right|=1$.

$$
\left\|\delta_{ \pm}-1\right\|_{L^{2}(d z)} \leq c \rho\left(1+\rho^{2}\right)^{\frac{1}{2}}\|r\|_{L^{2}} .
$$

We write $w=\left(w^{-}, w^{+}\right)$for a matrix $v$ and any of its factorization $v=\left(v^{-}\right)^{-1} v^{+}=\left(I-w^{-}\right)^{-1}\left(I+w^{+}\right), v^{ \pm},\left(v^{ \pm}\right)^{-1} \in L^{\infty}(\Sigma)$. Denote the singular integral operator $C_{w}(h)=C^{+}\left(h w^{-}\right)+C^{-}\left(h w^{+}\right)$acting on $L^{p}$-function valued matrices where $C^{ \pm}$is a limit of Cauchy operator $C$ from the (+)-side(respectively, (-)-side) of the oriented contour $\Sigma$. Note that if $w=(0, v-I)$ then $C_{w}=C_{v}$ as in (3.33). As shown in [DZ03, if $\left(1-C_{w}\right)^{-1}$ is bounded in $L^{p}$ for one factorization $v=$ $\left(1-w^{-1}\right)^{-1}\left(I+w^{+}\right)$, then it is bounded for all such factorizations.

Lemma 5.2. Let $v$ be as in (5.8), with $r \in H^{1,1}(\mathbb{R})$. Then for any factorization $v=\left(v^{-}\right)^{-1} v^{+}, v^{ \pm},\left(v^{ \pm}\right)^{-1} \in L^{\infty}(\mathbb{R})$, the operator $1-C_{w}$ with $w=\left(w^{-}, w^{+}\right)=\left(I-v^{-}, v^{+}-I\right)$ has a bounded inverse in $L^{2}(\mathbb{R})$ and

$$
\left\|\left(1-C_{w}\right)^{-1}\right\|_{L^{2}(\mathbb{R})} \leq c\left(1+\rho^{2}\right),
$$

where $\|r\|_{\infty} \leq \rho<\infty$. 
Proof. By Corollary 2.7 in DZ03, it is enough to show that $1-C_{v}$ is invertible in $L^{2}(\mathbb{R})$. We will first show that $1-C_{v}$ is a Fredholm operator of index 0 . Define $w=(0, v-I)$ and $\hat{w}=\left(0, v^{-1}-I\right)$. Then for $f \in L^{2}(\mathbb{R})$, using (3.32) and (3.33),

$$
\begin{aligned}
C_{w}\left(C_{\hat{w}} f\right) & =C^{-}\left[\left(C_{\hat{w}} f\right)(v-I)\right]=C^{-}\left[C^{-}\left(f\left(v^{-1}-I\right)\right)(v-I)\right] \\
& =C^{-}\left[\left(C^{+}\left(f\left(v^{-1}-I\right)\right)-f\left(v^{-1}-I\right)\right)(v-I)\right] \\
& =C^{-}\left[C^{+}\left(f\left(v^{-1}-I\right)\right)(v-I)+f\left(v+v^{-1}-2 I\right)\right] \\
& =C^{-}\left[C^{+}\left(f\left(v^{-1}-I\right)\right)(v-I)\right]+C_{w} f+C_{\hat{w}} f .
\end{aligned}
$$

As $v$ is continuous and $v-I \rightarrow 0$ as $|z| \rightarrow \infty$, it follows that we can approximate $v-I$ by a sequence of rational functions of the form,

$$
\sum_{i=1}^{n} \frac{B_{i}}{z-a_{i}}, \quad a_{i} \in \mathbb{C} \backslash \mathbb{R} .
$$

Let $h=f\left(v^{-1}-I\right)$. Then,

$$
\begin{aligned}
C^{-}\left(\left(C^{+} h\right)(\diamond) \frac{1}{\diamond-a_{i}}\right)(z) & =\lim _{\epsilon \downarrow 0} \int_{\mathbb{R}} \frac{\left(C^{+} h\right)(s)}{s-a_{i}} \frac{1}{s-(z-i \epsilon)} \frac{\mathrm{d} s}{2 \pi i} \\
& = \begin{cases}0 & \text { if } a_{i} \in \mathbb{C}^{-}, \\
\frac{C h\left(a_{i}\right)}{a_{i}-z} & \text { if } a_{i} \in \mathbb{C}^{+} .\end{cases}
\end{aligned}
$$

which implies that $f \mapsto C^{-}\left(\left(C^{+}\left(f\left(v^{-1}-I\right)\right)\right) \frac{1}{\diamond-a_{i}}\right)$ has either rank 0 or 1 and hence is compact in $L^{2}(\mathbb{R})$. It follows that $C^{-}\left[C^{+}\left(f\left(v^{-1}-I\right)\right)(v-\right.$ $I)]$ is operator limit of compact operators and hence is compact. But $\left(1-C_{w}\right)\left(1-C_{\hat{w}}\right)=1-C_{w}-C_{\hat{w}}+C_{w} C_{\hat{w}}=1+C^{-}\left[C^{+}\left(\diamond\left(v^{-1}-I\right)\right)(v-I)\right]$. Similarly, we see that $\left(1-C_{\hat{w}}\right)\left(1-C_{w}\right)-1$ is compact, and hence $1-C_{w}$ is a Fredholm operator. For $0 \leq \gamma \leq 1$, set $v(\gamma) \equiv\left(\begin{array}{c}1+\gamma^{2}|r|^{2} \gamma r e^{i \theta} \\ \bar{r} e^{-i \theta} \\ 1\end{array}\right)$. Clearly $v(1)=v$. The same argument shows that $v(\gamma)$ is Fredholm for all $\gamma \in[0,1]$. But for $\gamma=0, v(0)=I$ and so $1-C_{v(0)}=1$; we conclude that $\operatorname{ind}\left(1-C_{v(\gamma=1)}\right)=\operatorname{ind}\left(1-C_{v(\gamma=0)}\right)=0$. Thus $1-C_{v}$ is Fredholm of index zero. To complete the proof, we must show that $\left(1-C_{v}\right) f=0$, $f \in L^{2}(\mathbb{R})$, implies that $f=0$. Set $m_{ \pm}=C^{ \pm}(f(v-I)) \in \partial C\left(L^{2}\right)$. Then as $f=C_{v} f=C^{-}(f(v-I))=m_{-}$, we have

$$
\begin{aligned}
m_{+} & =C^{+}(f(v-I))=C^{-}(f(v-I))+f(v-I) \\
& =m_{-}+m_{-}(v-I)=m_{-} v .
\end{aligned}
$$

On the other hand, by Cauchy's theorem,

$$
0=\int_{\mathbb{R}} m_{+} m_{-}^{*} \mathrm{~d} z=\int_{\mathbb{R}} m_{-} v m_{-}^{*} \mathrm{~d} z .
$$


But $v$ is clearly strictly positive definite and hence $f=m_{-} \equiv 0$.

Suppose that $m_{ \pm} \in \partial C\left(L^{2}\right)$ and $m_{+}=m_{-} v+f$ for $f \in L^{2}(\mathbb{R})$. As above, we have

$$
0=\int_{\mathbb{R}} m_{+} m_{-}^{*} \mathrm{~d} z=\int_{\mathbb{R}} m_{-} v m_{-}^{*}+\int_{\mathbb{R}} f m_{-}^{*} .
$$

and hence

$$
\left|\int_{\mathbb{R}} m_{-} v m_{-}^{*}\right| \leq\|f\|_{L^{2}}\left\|m_{-}\right\|_{L^{2}} .
$$

But $v \geq \lambda_{0}$, where $\lambda_{0} \geq \frac{2+\rho^{2}-\sqrt{\left(2+\rho^{2}\right)^{2}-4}}{2}>0$ is the smallest eigenvalue of $v$. Hence $\lambda_{0}\left\|m_{-}\right\|_{L^{2}}^{2} \leq\|f\|_{L^{2}}\left\|m_{-}\right\|_{L^{2}}$ or $\left\|m_{-}\right\|_{L^{2}} \leq \frac{1}{\lambda_{0}}\|f\|_{L^{2}}$. But this bound then implies $\left\|\left(1-C_{w}\right)^{-1}\right\| \leq \frac{c}{\lambda_{0}}$, for any factorization $v=(I-$ $\left.w_{-}\right)^{-1}\left(I+w_{+}\right)$of $v$, by Proposition 2.6 and Corollary 2.7 in [DZ03].

Let $m_{\delta} \equiv m \delta^{-\sigma_{3}}$ solve the normalized $\operatorname{RHP}\left(\mathbb{R}, v_{\delta}\right)$ where $v_{\delta}=$ $\delta_{-}^{\sigma_{3}} v \delta_{+}^{-\sigma_{3}}$. In other words,

$$
\begin{aligned}
& w_{\delta}=\left(w_{\delta}^{-}, w_{\delta}^{+}\right) \\
& = \begin{cases}\left(\left(\begin{array}{cc}
0 & r \delta^{2} e^{i \theta} \\
0 & 0
\end{array}\right),\left(\begin{array}{cc}
0 & 0 \\
\bar{r} \delta^{-2} e^{-i \theta} & 0
\end{array}\right)\right), & z>z_{0}, \\
\left(\left(\begin{array}{cc}
0 & 0 \\
\frac{\bar{r}}{1+|r|^{2}} \delta_{-}^{-2} e^{-i \theta} & 0
\end{array}\right),\left(\begin{array}{cc}
0 & \frac{r}{1+|r|^{2}} \delta_{+}^{2} e^{i \theta} \\
0 & 0
\end{array}\right)\right), \quad z<z_{0} .\end{cases}
\end{aligned}
$$

Notation 5.3. We say that the bounds on two operators $A$ and $B$ in a Banach space are equivalent if and only if $c^{-1}\|B\| \leq\|A\| \leq c\|B\|$ for some $c>1$.

Lemma 5.4 (cf. DZ03, p. 1042). The operator $\left(1-C_{w_{\delta}}\right)^{-1}$ exists in $L^{2}(\mathbb{R})$ and the bound on $\left(1-C_{w_{\delta}}\right)^{-1}$ is equivalent to the bound on $\left(1-C_{w}\right)^{-1}$.

For a function $f$ on $\mathbb{R}$ we introduce a rational function which coincides with $f$ at $z_{0}$,

$$
[f](z)=\frac{f\left(z_{0}\right)}{\left(1+i\left(z-z_{0}\right)\right)^{2}}, \quad z \in \mathbb{R} .
$$

Replacing $r$ by the rational function $[r]$ etc., we obtain

$$
\begin{aligned}
& w_{\delta}^{[\cdot]}=\left(\left(w_{\delta}^{[\cdot]}\right)^{-},\left(w_{\delta}^{[\cdot]}\right)^{+}\right) \\
& \equiv \begin{cases}\left(\left(\begin{array}{cc}
0 & {[r] \delta^{2} e^{i \theta}} \\
0 & 0
\end{array}\right),\left(\begin{array}{cc}
0 & 0 \\
{[\bar{r}] \delta^{-2} e^{-i \theta}} & 0
\end{array}\right)\right), & z>z_{0}, \\
\left(\left(\begin{array}{cc}
0 & 0 \\
\left(\frac{\bar{r}}{1+|r|^{2}}\right] \delta_{-}^{-2} e^{-i \theta} & 0
\end{array}\right),\left(\begin{array}{cc}
0 & {\left[\frac{r}{1+|r|^{2}}\right] \delta_{+}^{2} e^{i \theta}} \\
0 & 0
\end{array}\right)\right), & z<z_{0} .\end{cases}
\end{aligned}
$$




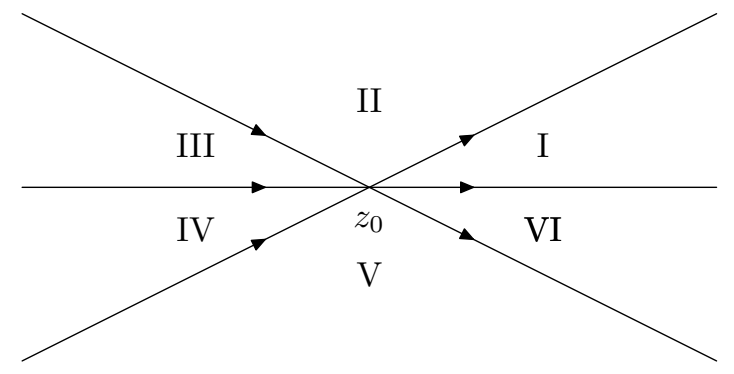

Figure 1. Contour $\Sigma^{e}$

Let $\Sigma^{e} \equiv \mathbb{R} \cup\left(z_{0}+e^{i \pi / 4} \mathbb{R}\right) \cup\left(z_{0}+e^{-i \pi / 4} \mathbb{R}\right)$ be oriented from left to right (see Figure 1).

Introduce the trivial extension $v_{e}^{[\cdot]}$ of $v_{\delta}^{[\cdot]}$ onto $\Sigma^{e}$ by setting $v_{e}^{[\cdot]}=v_{\delta}^{[\cdot]}$ on $\mathbb{R}$ and $v_{e}^{[\cdot]}=I$ on $\Sigma^{e} \backslash \mathbb{R}$.

Lemma 5.5 (cf. [DZ03], pp. 1043-1044). Suppose that $v_{e}^{[\cdot]}=(1-$ $\left.\left(w_{e}^{[\cdot]}\right)^{-}\right)^{-1}\left(1+\left(w_{e}^{[\cdot]}\right)^{+}\right)$is a factorization as above. Then, $\left(1-C_{w_{s}^{[\cdot]}}\right)^{-1}$ is bounded in $L^{2}(\mathbb{R})$ if and only if $\left(1-C_{w_{e}^{[]]}}\right)^{-1}$ is bounded in $L^{2}\left(\Sigma^{e}\right)$ and the bounds are equivalent.

Now, set

$$
\Phi=\left\{\begin{array}{lc}
I & \text { on II or V } \\
\left(\begin{array}{cc}
1 & 0 \\
-[\bar{r}] \delta^{-2} e^{-i \theta} & 1
\end{array}\right) & \text { on I } \\
\left(\begin{array}{cc}
1 & -\left[\frac{r}{1+|r|^{2}}\right] \delta^{2} e^{i \theta} \\
0 & 1
\end{array}\right) & \text { on III } \\
\left(\begin{array}{cc}
1 & 0 \\
{\left[\frac{\bar{r}}{1+|r|^{2}}\right] \delta^{-2} e^{-i \theta}} & 1
\end{array}\right) & \text { on IV } \\
\left(\begin{array}{cc}
1 & {[r] \delta^{2} e^{i \theta}} \\
0 & 1
\end{array}\right) & \text { on VI }
\end{array}\right.
$$

and set $v_{d}^{[\cdot]} \equiv \Phi_{-}^{-1} v_{e}^{[\cdot]} \Phi_{+}$where $\Phi \pm$ denote the $( \pm)$-limits on the contour $\Sigma^{e}$. Direct calculation shows that 


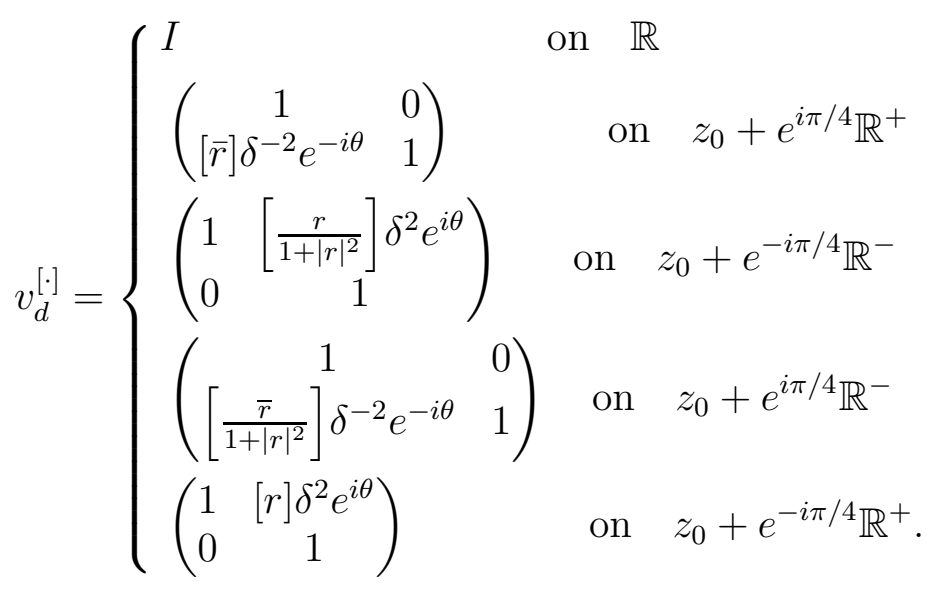

Because $\Phi$ is analytic and uniformly bounded in $\mathbb{C} \backslash \Sigma^{e}$ for all $x \in \mathbb{R}$, $t \geq 0$, we obtain the following.

Lemma 5.6 (cf. [DZ03], pp. 1044-1045). The operator $\left(1-C_{v_{d}^{[\cdot]}}\right)^{-1}$ is bounded if and only if $\left(1-C_{v_{e}^{[\cdot]}}\right)^{-1}$ is bounded in $L^{2}\left(\Sigma^{e}\right)$ and the bounds are equivalent.

The bound on, if it exists, is equivalent to the bound on.

As noted above, bounds on $\left(1-C_{v_{d}^{[\cdot]}}\right)^{-1}$ and $\left(1-C_{v_{e}^{[\cdot]}}\right)^{-1}$ imply bounds on $\left(1-C_{w_{d}^{[\cdot]}}\right)^{-1}$ and $\left(1-C_{w_{e}^{[\cdot]}}\right)^{-1}$ for any factorizations $v_{d}^{[\cdot]}=(1-$ $\left.\left(w_{d}^{[\cdot]}\right)^{-}\right)^{-1}\left(1+\left(w_{d}^{[\cdot]}\right)^{+}\right)$and $v_{e}^{[\cdot]}=\left(1-\left(w_{e}^{[\cdot]}\right)^{-}\right)^{-1}\left(1+\left(w_{e}^{[\cdot]}\right)^{+}\right)$. For $z<z_{0}$, define

$$
\begin{aligned}
& \beta\left(z, z_{0}\right) \equiv \\
& \int_{-\infty}^{z_{0}}\left\{\log \left(1+|r(s)|^{2}\right)-\log \left(1+\left|r\left(z_{0}\right)\right|^{2}\right) \chi^{0}(s)\left(s-z_{0}+1\right)\right\} \frac{d s}{2 \pi i(s-z)},
\end{aligned}
$$

where $\nu\left(z_{0}\right)=\frac{1}{2 \pi} \log \left(1+\left|r\left(z_{0}\right)\right|^{2}\right)$ and $\chi^{0}(s)$ denotes the characteristics function of the interval $\left(z_{0}-1, z_{0}\right)$. Define

$$
\delta_{0}(z) \equiv e^{\beta\left(z_{0}, z_{0}\right)-i \nu\left(z_{0}\right)}\left(z-z_{0}\right)^{-i \nu\left(z_{0}\right)}
$$


Set

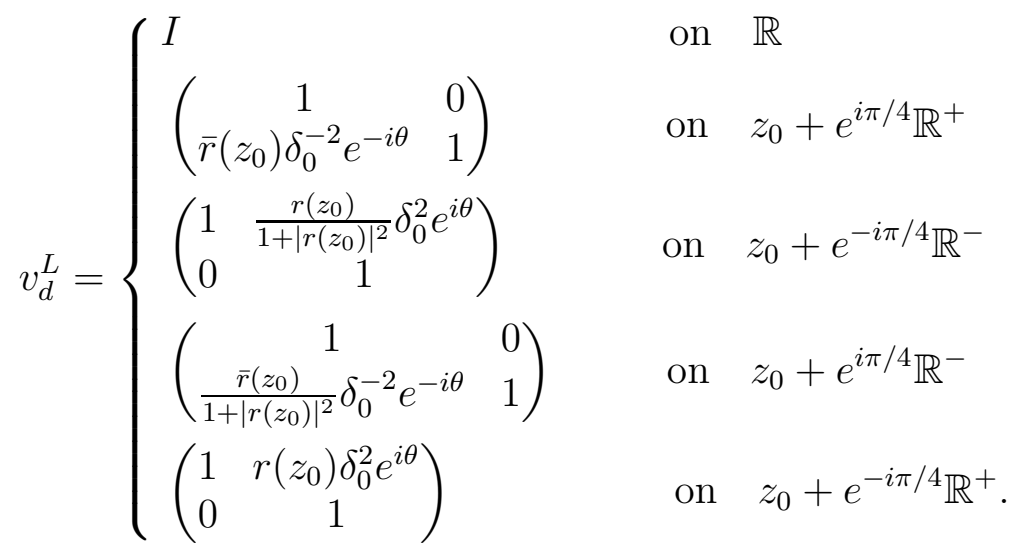

Lemma 5.7 (cf. [DZ03], Lemma 2.14). Let $r \in H^{1,0}(\mathbb{R})$ with $\|r\|_{H^{1,0}(\mathbb{R})} \leq$ $\lambda<\infty$. Then, for $1 \leq p \leq \infty$,

$$
\left\|v_{d}^{[\cdot]}-v_{d}^{L}\right\|_{L^{p}\left(\Sigma^{e}\right)} \leq \frac{c}{t^{\frac{1}{4}+\frac{1}{2 p}}}
$$

uniformly for $t \geq 1$ and all $x \in \mathbb{R}$ where $c$ depends on $\lambda$. Hence, $\left(1-C_{v_{d}^{[\cdot]}}\right)^{-1}$ is bounded if and only if $\left(1-C_{v_{d}^{L}}\right)^{-1}$ is bounded in $L^{2}\left(\Sigma^{e}\right)$ and the bounds are equivalent uniformly for $t \geq 1$ and all $x \in \mathbb{R}$.

Remark 5.8. If $r$ had more decay, say $r \in H^{1,1}(\mathbb{R})$, one sees by integration by part that

$$
\beta\left(z_{0}, z_{0}\right)-i \nu\left(z_{0}\right)=-\frac{1}{2 \pi i} \int_{-\infty}^{z_{0}} \log \left(z_{0}-s\right) d \log \left(1+|r(s)|^{2}\right) .
$$

Lemma 5.9 (cf. [DZ03], p. 1049). The operator $\left(1-C_{v_{d}^{L}}\right)^{-1}$ exists in $L^{2}\left(\Sigma^{e}\right)$ and its bound is equivalent to the bound on $\left(1-C_{v^{L}}\right)^{-1}$ in

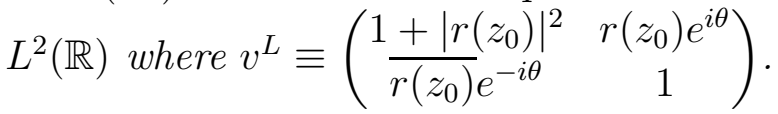

Proposition 5.10 (cf. [DZ03, Proposition 2.13). For all sufficiently large $t$, say $t \geq t_{0},\left(1-C_{w_{\delta}^{[\cdot]}}\right)^{-1}$ exists in $L^{2}(\mathbb{R})$, and for some uniform constant $c$

$$
\left\|\left(1-C_{w_{\delta}^{[\cdot]}}\right)^{-1}\right\|_{L^{2}(\mathbb{R})} \leq c \text { for } x \in \mathbb{R} \text { and } t \geq t_{0} .
$$

From the previous results, we see that for $t \geq t_{0},\left(1-C_{w_{\delta}^{[\cdot]}}\right)^{-1}$, $\left(1-C_{v_{e}^{[\cdot]}}\right)^{-1},\left(1-C_{v_{d}^{[\cdot]}}\right)^{-1}$ and $\left(1-C_{v_{d}^{L}}\right)^{-1}$ exist and bounded in $L^{2}$ and the bounds are equivalent. It follows that the normalized RHPs $\left(\mathbb{R}, v_{\delta}^{[\cdot]}\right),\left(\Sigma^{e}, v_{e}^{[\cdot]}\right),\left(\Sigma^{e}, v_{d}^{[\cdot]}\right)$ and $\left(\Sigma^{e}, v_{d}^{L}\right)$ have unique solutions $m_{\delta}^{[\cdot]}, m_{e}^{[\cdot]}$, $m_{d}^{[\cdot]}$ and $m_{d}^{L} \in I+\partial C\left(L^{2}\right)$, respectively. Note that

$$
m_{\delta}^{[\cdot]}=m_{d}^{[\cdot]} \Phi^{-1}
$$


For reasons that will become clear (see (5.21) below), we now reverse the orientation on $\mathbb{R}_{-}+z_{0}$ to obtain a contour $\widetilde{\mathbb{R}}_{z_{0}}=e^{i \pi}\left(\mathbb{R}^{+}+z_{0}\right) \cup$ $\left(\mathbb{R}^{+}+z_{0}\right)$ with associated jump matrix $\widetilde{v}_{\delta}=v_{\delta}$ for $z>z_{0}$ and $\widetilde{v}_{\delta}=v_{\delta}^{-1}$ for $z<z_{0}$. From the form of $v_{\delta}$, we see that $\widetilde{v}_{\delta}=\left(I-\widetilde{w}_{\delta}^{-}\right)^{-1}\left(I+\widetilde{w}_{\delta}^{+}\right)$ where

$$
\begin{aligned}
\widetilde{w}_{\delta}= & \left(\widetilde{w}_{\delta}^{-}, \widetilde{w}_{\delta}^{+}\right) \\
= & \begin{cases}\left(\left(\begin{array}{cc}
0 & r \delta^{2} e^{i \theta} \\
0 & 0
\end{array}\right),\left(\begin{array}{cc}
0 & 0 \\
\bar{r} \delta^{-2} e^{-i \theta} & 0
\end{array}\right)\right), & z>z_{0}, \\
\left(\left(\begin{array}{cc}
0 & -\frac{r}{1+|r|^{2}} \tilde{\delta}_{-}^{2} e^{i \theta} \\
0 & 0
\end{array}\right),\left(\begin{array}{cc}
0 & 0 \\
-\frac{r}{1+|r|^{2}} \tilde{\delta}_{+}^{-2} e^{-i \theta} & 0
\end{array}\right)\right), \quad z<z_{0} .\end{cases}
\end{aligned}
$$

where $\tilde{\delta}_{ \pm}(z)$ denotes the boundary values of $\delta(z)$ on $\widetilde{\mathbb{R}}_{z_{0}}$. Thus $\tilde{\delta}_{ \pm}(z)=$ $\delta_{ \pm}(z)$ for $z>z_{0}$ and $\tilde{\delta}_{ \pm}(z)=\delta_{\mp}(z)$ for $z<z_{0}$.

We use $\rho, \lambda$ and $\eta$ to denote $L^{\infty}, H^{1,0}$ and $H^{1,1}$ bounds for $r$ respectively. Thus

$$
\|r\|_{L^{\infty}} \leq \rho, \quad\|r\|_{H^{1,0}} \leq \lambda, \quad\|r\|_{H^{1,1}} \leq \eta
$$

Extend $\widetilde{\mathbb{R}}_{z_{0}}$ to a contour $\Gamma_{z_{0}}=\widetilde{\mathbb{R}}_{z_{0}} \cup\left(z_{0}+e^{i \pi / 2} \mathbb{R}_{-}\right) \cup\left(z_{0}+e^{-i \frac{\pi}{2}} \mathbb{R}_{-}\right)$. As a complete contour, $\Gamma_{z_{0}}$ has the important property

$$
C_{\Gamma_{z_{0}}}^{+} C_{\Gamma_{z_{0}}}^{-}=C_{\Gamma_{z_{0}}}^{-} C_{\Gamma_{z_{0}}}^{+}=0
$$

In the following lemmas we will assume for convenience that $x=0$. Thus $z_{0}=0, \delta=\delta_{z_{0}=0}, \Delta=\Delta_{z_{0}=0}, \widetilde{\mathbb{R}} \equiv \widetilde{\mathbb{R}}_{z_{0}=0}, \Gamma \equiv \widetilde{\Gamma}_{z_{0}=0}$, and $\theta=-t z^{2} / 2$.

Lemma 5.11 (cf. [DZ03], Lemma 4.1). For $z \in \mathbb{R} \backslash 0$,

$$
\left|\Delta^{\prime}(z)\right| \leq \mathrm{I}+\mathrm{II}
$$

where

$$
\begin{aligned}
& \|\mathrm{I}\|_{L^{2}} \leq c \rho \lambda \\
& |\mathrm{II}| \leq c \rho^{2} \frac{1}{|z|} .
\end{aligned}
$$

Lemma 5.12 ([DZ03], p.1064). Suppose $f \in H^{1,0}$. Then for all $t>1$,

$$
\left|\int_{\mathbb{R}} f \Delta^{ \pm 1} e^{\mp i t z^{2}} d z\right| \leq \frac{c(1+\rho \lambda)}{t^{\frac{1}{2}}}\|f\|_{H^{1,0}} .
$$

In addition, if $f(0)=0$, then for all $t>1$,

$$
\left|\int_{\mathbb{R}} f \Delta^{ \pm 1} e^{\mp i t z^{2}} d z\right| \leq \frac{c(1+\rho \lambda)}{t^{3 / 4}}\|f\|_{H^{1,0}} .
$$




\begin{tabular}{l|l}
$D_{2}$ & $D_{1}$ \\
\hline$D_{3}$ & $D_{4}$
\end{tabular}

Figure 2. Contour $\Gamma$

Let $D_{j}, j=1, \ldots, 4$, be the $j^{\text {th }}$ quadrant in $\mathbb{C} \backslash \Gamma$ (see Figure 2). In the Lemma below $H^{q}$ denotes Hardy space.

Lemma 5.13 ([DZ03], pp.1065-1069). Suppose $f \in H^{1,0}$, then for $2 \leq p<\infty$ and for all $t \geq 0$,

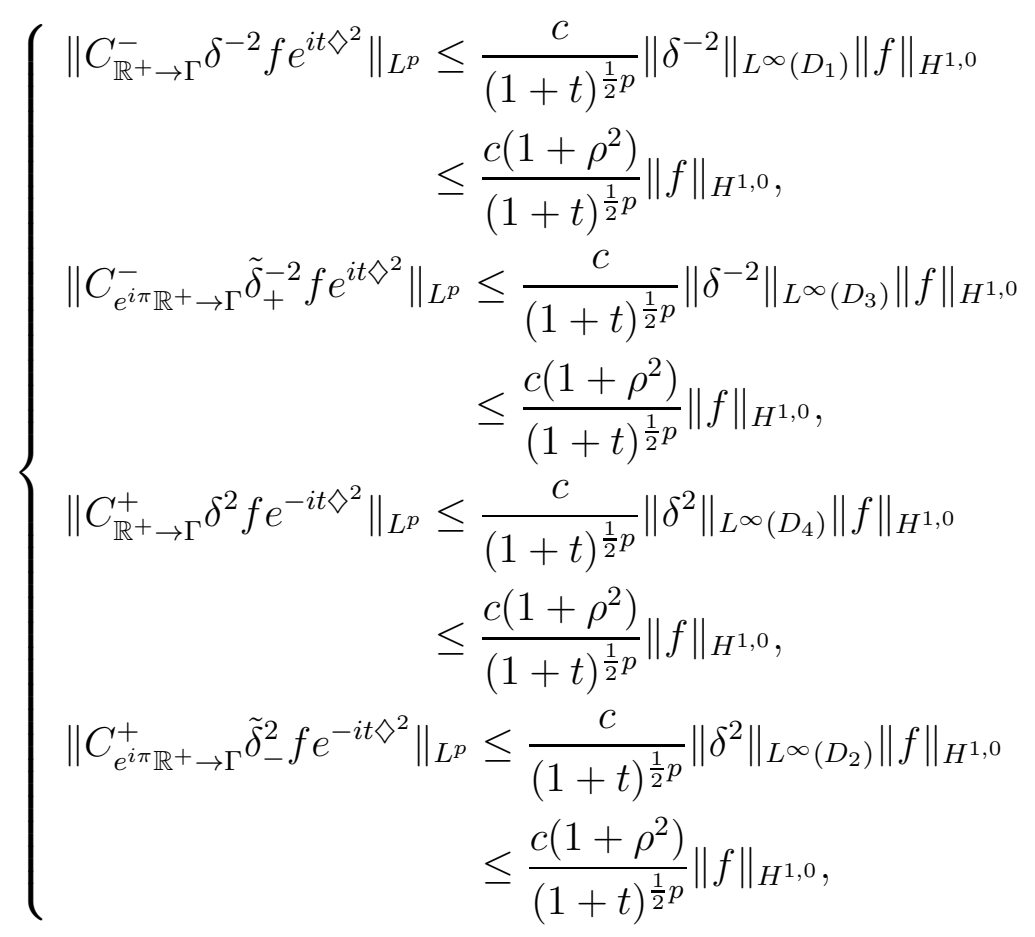

Suppose in addition that $f(0)=0$ and that $g$ is a function in the Hardy space $H^{q}(\mathbb{C} \backslash \mathbb{R})$ for some $2 \leq q \leq \infty$. Then for all $t \geq 0$,

$$
\left\{\begin{array}{l}
\left\|C_{\mathbb{R}^{+} \rightarrow \Gamma}^{-} g_{+} f e^{i t \diamond^{2}}\right\|_{L^{2}} \leq \frac{c}{(1+t)^{\frac{1}{2}-\frac{1}{q}}}\|g\|_{H^{q}(\mathbb{C} \backslash \mathbb{R})}\|f\|_{H^{1,0}}, \\
\left\|C_{e^{i \pi} \mathbb{R}^{+} \rightarrow \Gamma}^{-} \tilde{g}_{+} f e^{i t \diamond^{2}}\right\|_{L^{2}} \leq \frac{c}{(1+t)^{\frac{1}{2}-\frac{1}{q}}}\|g\|_{H^{q}(\mathbb{C} \backslash \mathbb{R})}\|f\|_{H^{1,0}}, \\
\left\|C_{\mathbb{R}^{+} \rightarrow \Gamma}^{+} g_{-} f e^{-i t \diamond^{2}}\right\|_{L^{2}} \leq \frac{c}{(1+t)^{\frac{1}{2}-\frac{1}{q}}}\|g\|_{H^{q}(\mathbb{C} \backslash \mathbb{R})}\|f\|_{H^{1,0}}, \\
\left\|C_{e^{i \pi} \mathbb{R}^{+} \rightarrow \Gamma}^{+} \tilde{g}_{-} f e^{-i t \diamond^{2}}\right\|_{L^{2}} \leq \frac{c}{(1+t)^{\frac{1}{2}-\frac{1}{q}}}\|g\|_{H^{q}(\mathbb{C} \backslash \mathbb{R})}\|f\|_{H^{1,0}},
\end{array}\right.
$$


where $g_{ \pm}$are the boundary values of $g$ on $\mathbb{R}$ and $\tilde{g}_{ \pm}=g_{\mp}$ on $e^{i \pi} \mathbb{R}^{+}$.

Since the $H^{1,0}$ - and $L^{\infty}$-norms of $r(z)$ is invariant under translation, appropriate choices of $f$ in (5.24) give rise to the following.

Corollary 5.14 (cf. DZ03, Corollary 4.4). For any $2 \leq p<\infty$, and for all $t \geq 0$

$$
\left\|C_{\widetilde{\mathbb{R}}_{z_{0}} \rightarrow \Gamma_{z_{0}}}^{ \pm} \widetilde{w}_{\delta}^{ \pm}\right\|_{L^{p}\left(\Gamma_{z_{0}}\right)} \leq \frac{c \lambda\left(1+\rho^{2}\right)}{(1+t)^{\frac{1}{2} p}} .
$$

We need the following $L^{p}$ bound on solutions of RHPs.

Proposition 5.15 (cf. [DZ03], Proposition 4.5). Suppose that $r$ is a continuous function on $\mathbb{R}$ such that

$$
\lim _{z \rightarrow \infty} r(z)=0, \quad \text { and }\|r\|_{L^{\infty}(\mathbb{R})} \leq \rho<\infty .
$$

Let $v$ be given as in (5.8) and $v=\left(1-w^{-}\right)^{-1}\left(1+w^{+}\right)$is any factorization as above. Then for any $p \geq 2$, there exists $t_{0}=t_{0}(r, p)$ such that for $t \geq t_{0}$ and all $x \in \mathbb{R},\left(1-C_{w}\right)^{-1}$ exists in $L^{p}(\mathbb{R})$ and

$$
\left\|\left(1-C_{w}\right)^{-1}\right\|_{L^{p}(\mathbb{R}) \rightarrow L^{p}(\mathbb{R})} \leq c
$$

for $t \geq t_{0}$ and all $x \in \mathbb{R}$.

By Lemma 5.4 and Proposition 5.15, we obtain for $r \in H^{1,0}$ and $2 \leq p<\infty$,

$$
\left\|\left(1-C_{\widetilde{w}_{\delta}}\right)^{-1}\right\|_{L^{p}\left(\widetilde{\mathbb{R}}_{z_{0}}\right) \rightarrow L^{p}\left(\widetilde{\mathbb{R}}_{z_{0}}\right)} \leq c_{p}
$$

where $c_{p}$ is uniform for all $x \in \mathbb{R}$ and all $t \geq t_{0}$. Reversing the orientation on $\mathbb{R}$ as above, we see that (cf. Proposition 2.8 in [DZ03])

$$
\left\|\left(1-C_{\widetilde{w}_{\delta}}\right)^{-1}\right\|_{L^{p}\left(\widetilde{\mathbb{R}}_{z_{0}}\right) \rightarrow L^{p}\left(\widetilde{\mathbb{R}}_{z_{0}}\right)} \leq c_{p}^{\prime},
$$

where $c_{p}^{\prime}$ is uniform for all $x \in \mathbb{R}$ and all $t \geq t_{0}$. Let $\widetilde{\mu}_{\delta} \in I+L^{2}(\widetilde{\mathbb{R}})$ be the solution of $\left(1-C_{\widetilde{w}_{\delta}}\right) \widetilde{\mu}_{\delta}=I$. Using Corollary 5.14 and (5.26), we obtain the following result.

Corollary 5.16 (cf. [DZ03], Corollary 4.6). For any $2 \leq p<\infty$ and for all $t \geq 0$

$$
\left\|\widetilde{\mu}_{\delta}-I\right\|_{L^{p}} \leq \frac{c_{p} \lambda\left(1+\rho^{2}\right)}{(1+t)^{\frac{1}{2} p}} .
$$

Lemma 5.17 (cf. DZ03, Lemma 4.7). For any $2 \leq p<\infty$ and for $t$ sufficiently large,

$$
\left\|C^{ \pm} \widetilde{\mu}_{\delta}\left(\widetilde{w}_{\delta}^{[\cdot] \pm}-\widetilde{w}_{\delta}^{\mp}\right)\right\|_{L^{2}} \leq c t^{-\frac{1}{2}+\frac{1}{2 p}}
$$


Lemma 5.18 (cf. DZ03, Lemma 4.8). For any $2 \leq p<\infty$ and for $t$ sufficiently large,

$$
\left|\int_{\widetilde{\mathbb{R}}} \widetilde{\mu}_{\delta} \widetilde{w}_{\delta}-\int_{\widetilde{\mathbb{R}}} \widetilde{\mu}_{\delta}^{[\cdot]} \widetilde{w}_{\delta}^{[\cdot]}\right| \leq c t^{-\frac{3}{4}+\frac{1}{2 p}}
$$

and for $z \in \mathbb{C} \backslash \mathbb{R}$

$$
\left|\widetilde{m}_{\delta}(z)-\widetilde{m}_{\delta}^{[\cdot]}(z)\right| \leq c(z) t^{-\frac{3}{4}+\frac{1}{2 p}}
$$

where $c(z)$ is a constant depending on $z$.

Proof. The proof of (5.27) follows the proof of Lemma 4.8 in [DZ03].

The proof of (5.28) is similar using the relation $\widetilde{m}_{\delta}(z)=I+\int \frac{\widetilde{\mu}_{\delta}(s) \widetilde{w}_{\delta}(s)}{s-z} \frac{d s}{2 \pi i}$, and $\widetilde{m}_{\delta}^{[\cdot]}(z)=I+\int \frac{\widetilde{\mu}_{\delta}^{[\cdot]}(s) \widetilde{w}_{\delta}^{[\cdot]}(s)}{s-z} \frac{d s}{2 \pi i}$.

Since $\Phi=I$ in the region II, $\widetilde{m}_{\delta}^{[\cdot]}=m_{\delta}^{[\cdot]}=m_{d}^{[\cdot]} \Phi^{-1}=m_{d}^{[\cdot]}$ on II and hence the residues at $z=\infty$ are the same, i.e. $\lim _{z \rightarrow \infty, z \in \mathrm{II}} z \widetilde{m}_{\delta}^{[\cdot]}=$ $\lim _{z \rightarrow \infty, z \in \mathrm{II}} z m_{d}^{[\cdot]}$, which implies $\int_{\widetilde{\mathbb{R}}} \widetilde{\mu}_{\delta}^{[\cdot]} \widetilde{w}_{\delta}^{[\cdot]}=\int_{\Sigma^{e}} \mu_{d}^{[\cdot]} w_{d}^{[\cdot]}$.

Lemma 5.19 (cf. [DZ03], pp. 1073-1074). For t sufficiently large,

$$
\left|\int_{\Sigma^{e}} \mu_{d}^{L} w_{d}^{L}-\int_{\Sigma^{e}} \mu_{d}^{[\cdot] \cdot]} w_{d}^{[\cdot]}\right| \leq c t^{-\frac{3}{4}}
$$

and for $z \in \mathbb{C} \backslash \mathbb{R}$,

$$
\left|m_{d}^{L}(z)-m_{d}^{[\cdot]}(z)\right| \leq c(z) t^{-\frac{3}{4}}
$$

where $c(z)$ is a constant depending on $z$.

Proof. As in Lemma 5.18, the proof of (5.30) is similar to the proof of (5.29).

If $m_{1}$ and $\delta_{1}$ are the residues at $z=\infty$ of $m(z)=m(x, t, z)$ and $\delta(z)^{\sigma_{3}}$ respectively, we see that

$$
m_{1}(x, t)=-\frac{1}{2 \pi i} \int_{\mathbb{R}} \mu w \quad \text { and } \quad \delta_{1}(x, t)=-\frac{1}{2 \pi i} \int_{-\infty}^{z_{0}} \log \left(1+|r|^{2}\right) \sigma_{3} .
$$

Since $m_{\delta}(z)=\widetilde{m}_{\delta}(z)=m(z) \delta(z)^{-\sigma_{3}}$, the residue $\left(\widetilde{m}_{\delta}\right)_{1}(x, t)$ at $z=\infty$ of $\widetilde{m}_{\delta}(x, t, z)$ is given by $\left(\widetilde{m}_{\delta}\right)_{1}=m_{1}-\delta_{1}$. Assembling the above results, we conclude that for any $p \geq 2$ and all sufficiently large $t$,

$$
\begin{aligned}
u_{f}(x, t) & =-i\left(m_{1}(x, t)\right)_{12}=-i\left(\left(\widetilde{m}_{\delta}\right)_{1}(x, t)\right)_{12} \\
& =\frac{1}{2 \pi} \int_{\widetilde{\mathbb{R}}}\left(\widetilde{\mu}_{\delta} \widetilde{w}_{\delta}\right)_{12}=\frac{1}{2 \pi} \int_{\Sigma^{e}}\left(\mu_{d}^{L} w_{d}^{L}\right)_{12}+O\left(t^{-\frac{3}{4}+\frac{1}{2 p}}\right) \\
& =-i\left(\left(m_{d}^{L}\right)_{1}(x, t)\right)_{12}+O\left(t^{-\frac{3}{4}+\frac{1}{2 p}}\right),
\end{aligned}
$$


where $\left(m_{d}^{L}\right)_{1}$ is the residue of $m_{d}^{L}$ at $z=\infty$. Similarly, for any fixed $z \in \mathbb{C} \backslash \mathbb{R}$

$$
\begin{aligned}
m(z) & =\widetilde{m}_{\delta}(z) \delta^{\sigma_{3}}(z) \\
& =\widetilde{m}_{\delta}^{[\cdot]}(z) \delta^{\sigma_{3}}(z)+O\left(t^{-\frac{3}{4}+\frac{1}{2 p}}\right) \\
& =m_{d}^{[\cdot]}(z) \Phi^{-1}(z) \delta^{\sigma_{3}}(z)+O\left(t^{-\frac{3}{4}+\frac{1}{2 p}}\right) \\
& =m_{d}^{L}(z) \Phi^{-1}(z) \delta^{\sigma_{3}}(z)+O\left(t^{-\frac{3}{4}+\frac{1}{2 p}}\right) .
\end{aligned}
$$

Remark 5.20. Clearly by the above estimates, the error terms in (5.31) and (5.32) are controlled by $H^{1,0}$ norm of the reflection coefficient function $r(z)$. In particular, if $\|r\|_{L^{\infty}} \leq c \epsilon, \epsilon \ll 1$, then it is easy to check that the error terms in (5.31) and (5.32) are $O\left(\|r\|_{H^{1,0}} t^{-\frac{3}{4}+\frac{1}{2 p}}\right)$, $O\left(\left\|\frac{r(\cdot)}{\cdot-z}\right\|_{H^{1,0}} t^{-\frac{3}{4}+\frac{1}{2 p}}\right)$, respectively.

If $\|r\|_{H^{1,0}}$ is sufficiently small, then the singular operators $\left(1-C_{w_{\delta}^{[\cdot]}}\right)^{-1}$, etc. are bounded uniformly on $t \geq 0$, which leads the following result.

Lemma 5.21. If $r(z)$ has sufficiently small norm in $H^{1,0}$, we have for all $t \geq 1$,

$$
u_{f}(x, t)=-i\left(\left(m_{d}^{L}\right)_{1}(x, t)\right)_{12}+O\left(\|r\|_{H^{1,0}} t^{-\frac{3}{4}+\frac{1}{2 p}}\right)
$$

and for any fixed $z \in \mathbb{C} \backslash \mathbb{R}$,

$$
m(z)=m_{d}^{L}(z) \Phi^{-1}(z) \delta^{\sigma_{3}}(z)+O\left(\left\|\frac{r(\cdot)}{\cdot-z}\right\|_{H^{1,0}} t^{-\frac{3}{4}+\frac{1}{2 p}}\right) .
$$

\section{LOCALIZED RHP}

We compute the solution of the localized $\operatorname{RHP}\left(\Sigma^{e}, v_{d}^{L}\right)$ explicitly in terms of parabolic cylinder functions (see AS65]). Introduce the scaling $\zeta=\sqrt{t}\left(z-z_{0}\right)$ and set $($ see $(\underline{5.17}))$

$$
\phi(\zeta)=\delta_{0}^{2}(z) e^{i \theta(z)}=\alpha_{0}^{2} \zeta^{-2 i \nu\left(z_{0}\right)} e^{-i \zeta^{2} / 2}
$$

where

$$
\alpha_{0}=\exp \left[\frac{i x^{2}}{4 t}+\frac{i}{2} \nu\left(z_{0}\right) \log t+\beta\left(z_{0}, z_{0}\right)-i \nu\left(z_{0}\right)\right]
$$

Define

$$
M_{d}^{L}(x, t, \zeta) \equiv m_{d}^{L}(x, t, z), \quad M^{\infty}(\zeta) \equiv M_{d}^{L}(\zeta) \phi^{\sigma}(\zeta), \quad z \in \mathbb{C} \backslash \Sigma^{e}
$$




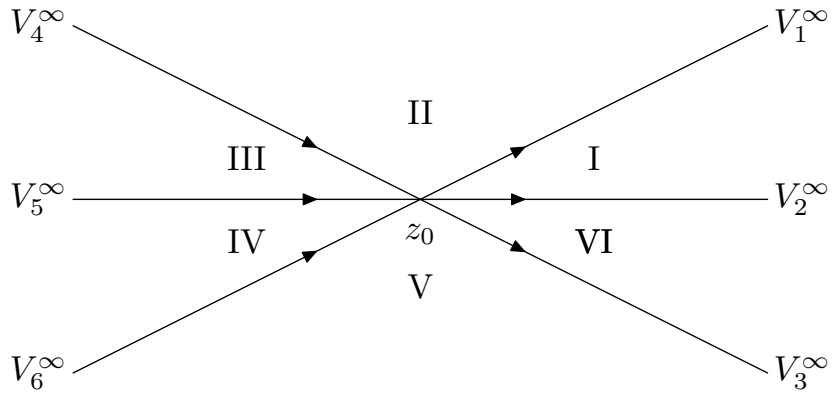

FiguRE 3. Numbering of $V^{\infty}$ on $\Sigma^{e}$

and

$$
\begin{aligned}
V^{\infty} \equiv & \phi(\zeta)^{-\mathrm{ad} \sigma} v_{d}^{L}(z) \\
& = \begin{cases}I & \text { on } z_{0}+\mathbb{R}^{+} \\
\left(\begin{array}{cc}
1 & 0 \\
\bar{r}\left(z_{0}\right) & 1
\end{array}\right) & \text { on } z_{0}+e^{i \pi / 4} \mathbb{R}^{+} \\
\left(\begin{array}{cc}
1+\left|r\left(z_{0}\right)\right|^{2} & 0 \\
0 & \frac{1}{1+\left|r\left(z_{0}\right)\right|^{2}}
\end{array}\right) & \text { on } z_{0}+\mathbb{R}^{-} \\
\left(\begin{array}{cc}
1 & \frac{r\left(z_{0}\right)}{1+\left|r\left(z_{0}\right)\right|^{2}} \\
0 & 1
\end{array}\right) & \text { on } z_{0}+e^{-i \pi / 4} \mathbb{R}^{-} \\
\left(\begin{array}{cc}
1 & 0 \\
\frac{\bar{r}\left(z_{0}\right)}{1+\left|r\left(z_{0}\right)\right|^{2}} & 1
\end{array}\right) & \text { on } z_{0}+e^{i \pi / 4} \mathbb{R}^{-} \\
\left(\begin{array}{cc}
1 & r\left(z_{0}\right) \\
0 & 1
\end{array}\right) & \text { on } z_{0}+e^{-i \pi / 4} \mathbb{R}^{+} .\end{cases}
\end{aligned}
$$

Then, it follows that $M_{+}^{\infty}(\zeta)=M_{-}^{\infty}(\zeta) V^{\infty}$ on $\zeta \in \Sigma^{e}-z_{0}$. Since $\operatorname{det} V_{d}^{L} \equiv 1$ on $\Sigma^{e}, \operatorname{det} M_{d}^{L}(z) \equiv 1$ (see Remark [3.8), $\operatorname{det} M^{\infty}=$ $\operatorname{det}\left(M_{d}^{L} \phi^{-\sigma}\right)=1$, and hence $\left(M_{d}^{L}\right)^{-1},\left(M_{\infty}\right)^{-1}$ exist. Numbering the restrictions of piecewise constant jump matrix $V^{\infty}$ on $\Sigma^{e}$ as in Figure 3, we have the cyclic condition $V_{3}^{\infty} V_{2}^{\infty} V_{1}^{\infty}=V_{6}^{\infty} V_{5}^{\infty} V_{4}^{\infty}$, which implies, in particular (see e.g. [BDT88]), that in each sector I, II, $\cdots, \mathrm{VI}, M^{\infty}(\zeta)$ is the restriction of an entire function. In particular this implies that $M^{\infty}$ is differentiable with respect to $\zeta$ and $\partial_{\zeta} M_{+}^{\infty}(\zeta)=\partial_{\zeta} M_{-}^{\infty}(\zeta) V^{\infty}$ on $\zeta \in \Sigma^{e}-z_{0}$. Thus, $\left(\partial_{\zeta} M^{\infty}\right)\left(M^{\infty}\right)^{-1}$ has a trivial jump matrix and 
hence is entire. As $\zeta \rightarrow \infty, \zeta \in \mathbb{C} \backslash\left(\Sigma^{e}-z_{0}\right)$,

$$
\begin{aligned}
\partial_{\zeta} M^{\infty}\left(M^{\infty}\right)^{-1} & =\partial_{\zeta}\left(M_{d}^{L} \phi^{\sigma}\right)\left(\phi^{-\sigma}\left(M_{d}^{L}\right)^{-1}\right) \\
& =\left[\partial_{\zeta} M_{d}^{L} \phi^{\sigma}+M_{d}^{L}\left(\frac{-2 i \nu\left(z_{0}\right) \sigma}{\zeta}-i \zeta \sigma\right) \phi^{\sigma}\right]\left(\phi^{-\sigma}\left(M_{d}^{L}\right)^{-1}\right) \\
& =M_{d}^{L}(-i \zeta \sigma)\left(M_{d}^{L}\right)^{-1}+O\left(\frac{1}{\zeta}\right) .
\end{aligned}
$$

Here, we have used the fact that $\partial_{\zeta} M_{d}^{L}=O\left(\zeta^{-1}\right)$. As $M_{d}^{L}=I+\frac{\left(M_{d}^{L}\right)_{1}}{\zeta}+$ $o\left(\zeta^{-1}\right)$ as $\zeta \rightarrow \infty$, we have

$$
\begin{aligned}
\hat{M} & \equiv\left(\partial_{\zeta} M^{\infty}+i \zeta \sigma M^{\infty}\right)\left(M^{\infty}\right)^{-1}=\partial_{\zeta} M^{\infty}\left(M^{\infty}\right)^{-1}+i \zeta \sigma \\
& =i \zeta\left[\sigma, M_{d}^{L}\right]\left(M_{d}^{L}\right)^{-1}+O\left(\frac{1}{\zeta}\right) \\
& =i \zeta\left[\sigma, I+\frac{\left(M_{d}^{L}\right)_{1}}{\zeta}+o\left(\frac{1}{\zeta}\right)\right]\left(I-\frac{\left(M_{d}^{L}\right)_{1}}{\zeta}+o\left(\frac{1}{\zeta}\right)\right)+O\left(\frac{1}{\zeta}\right) \\
& =i\left[\sigma,\left(M_{d}^{L}\right)_{1}\right]+o(1) .
\end{aligned}
$$

But $\hat{M}$ has no jumps across $\Sigma^{e}$ and hence by Liouville's theorem, $\hat{M} \equiv$ $i\left[\sigma,\left(M_{d}^{L}\right)_{1}\right]$. Then, from the definition of $\hat{M}$, we obtain the following system of ODEs

$$
\left(\partial_{\zeta} M^{\infty}+i \zeta \sigma M^{\infty}\right)=i\left[\sigma,\left(M_{d}^{L}\right)_{1}\right] M^{\infty} .
$$

Set

$$
\left(\begin{array}{cc}
0 & k_{1} \\
k_{2} & 0
\end{array}\right)=i\left[\sigma,\left(M_{d}^{L}\right)_{1}\right]
$$

The differential equations for $M_{11}^{\infty}$ and $M_{21}^{\infty}$ are given by

$$
\left\{\begin{array}{l}
\partial_{\zeta} M_{11}^{\infty}+\frac{i \zeta}{2} M_{11}^{\infty}=k_{1} M_{21}^{\infty}, \\
\partial_{\zeta} M_{21}^{\infty}-\frac{i \zeta}{2} M_{21}^{\infty}=k_{2} M_{11}^{\infty} .
\end{array}\right.
$$

Then,

$$
\begin{aligned}
k_{1} k_{2} M_{11}^{\infty} & =\partial_{\zeta}\left(\partial_{\zeta} M_{11}^{\infty}+\frac{i}{2} \zeta M_{11}^{\infty}\right)-\frac{i \zeta}{2}\left(\partial_{\zeta} M_{11}^{\infty}+\frac{i}{2} \zeta M_{11}^{\infty}\right) \\
& =\partial_{\zeta}^{2} M_{11}^{\infty}+\frac{\zeta^{2}}{4} M_{11}^{\infty}+\frac{i}{2} M_{11}^{\infty}
\end{aligned}
$$

Set $\eta \equiv e^{-\frac{3 i \pi}{4}} \zeta$ and $g(\eta) \equiv M_{11}^{\infty}(\zeta)$. Then, we see that $g$ satisfies the parabolic cylinder equation

$$
\partial_{\eta}^{2} g+\left(\frac{1}{2}-\frac{\eta^{2}}{4}+a\right) g=0, \text { where } a=i k_{1} k_{2} .
$$


General solutions of this equation can be written in terms of standard entire parabolic cylinder functions $D_{a}(\cdot)$ as follows.

$$
M_{11}^{\infty}(\zeta)=c_{1} D_{a}\left(e^{-\frac{3 i \pi}{4}} \zeta\right)+c_{2} D_{a}\left(-e^{-\frac{3 i \pi}{4}} \zeta\right)
$$

Since $M_{d}^{L}(\zeta) \rightarrow I$ as $\zeta \rightarrow \infty, z \in i \mathbb{R}$, we have

$$
\begin{aligned}
\left(M_{+}^{\infty}\right)_{11}(\zeta) & =(1+o(1)) \phi_{11}^{\sigma} \\
& =(1+o(1)) \alpha_{0} e^{\frac{3 \pi}{4} \nu\left(z_{0}\right)} \eta^{-i \nu\left(z_{0}\right)} e^{-\frac{1}{4} \eta^{2}}
\end{aligned}
$$

But from [AS65], as $\eta \rightarrow \infty$,

$$
D_{a}(\eta)=\left\{\begin{array}{c}
\eta^{a} e^{-\frac{1}{4} \eta^{2}}\left(1+O\left(a \eta^{-2}\right)\right) \\
\text { for }|\arg \eta|<\frac{3 \pi}{4} \\
\eta^{a} e^{-\frac{1}{4} \eta^{2}}\left(1+O\left(a \eta^{-2}\right)\right) \\
-(2 \pi)^{\frac{1}{2}}(\Gamma(-a))^{-1} e^{a \pi i} \eta^{-a-1} e^{\frac{\eta^{2}}{4}}\left(1+O\left(a \eta^{-2}\right)\right), \\
\text { for } \frac{\pi}{4}<\arg \eta<\frac{5 \pi}{4} \\
\eta^{a} e^{-\frac{1}{4} \eta^{2}}\left(1+O\left(a \eta^{-2}\right)\right) \\
-(2 \pi)^{\frac{1}{2}}(\Gamma(-a))^{-1} e^{-a \pi i} \eta^{-a-1} e^{\frac{\eta^{2}}{4}}\left(1+O\left(a \eta^{-2}\right)\right), \\
\text { for }-\frac{5 \pi}{4}<\arg \eta<-\frac{\pi}{4}
\end{array}\right.
$$

Inserting (6.8) into (6.6) and comparing with (6.7), we see that $a=$ $-i \nu\left(z_{0}\right)$, and $c_{1}=\alpha_{0} e^{\frac{3 \pi}{4} \nu\left(z_{0}\right)}$. Utilizing (6.5), we thus have for $\zeta \in$ $\mathrm{II}-z_{0} \equiv \mathrm{II}_{z_{0}}$

$$
\begin{aligned}
& M_{11}^{\infty}(\zeta)=\alpha_{0} e^{\frac{3 \pi}{4} \nu\left(z_{0}\right)} D_{a}\left(e^{-\frac{3 i \pi}{4}} \zeta\right) \\
& M_{21}^{\infty}(\zeta)=\frac{\alpha_{0}}{k_{1}} e^{\frac{3 \pi}{4} \nu\left(z_{0}\right)}\left[\partial_{\zeta}\left(D_{a}\left(e^{-\frac{3 i \pi}{4}} \zeta\right)\right)+\frac{i \zeta}{2} D_{a}\left(e^{-\frac{3 i \pi}{4}} \zeta\right)\right],
\end{aligned}
$$

Similarly, we have for $\zeta \in \mathrm{II}_{z_{0}}$,

$$
\begin{aligned}
& M_{12}^{\infty}(\zeta)=\frac{\alpha_{0}^{-1}}{k_{2}} e^{-\frac{\pi}{4} \nu\left(z_{0}\right)}\left[\partial_{\zeta}\left(D_{-a}\left(e^{-\frac{i \pi}{4}} \zeta\right)\right)-\frac{i \zeta}{2} D_{-a}\left(e^{-\frac{i \pi}{4}} \zeta\right)\right] \\
& M_{22}^{\infty}(\zeta)=\alpha_{0}^{-1} e^{-\frac{\pi}{4} \nu\left(z_{0}\right)} D_{-a}\left(e^{-\frac{i \pi}{4}} \zeta\right) .
\end{aligned}
$$


Also, for $\zeta \in \mathrm{V}_{z_{0}}$,

$$
\begin{aligned}
& M_{11}^{\infty}(\zeta)=\alpha_{0} e^{-\frac{\pi}{4} \nu\left(z_{0}\right)} D_{a}\left(e^{\frac{i \pi}{4}} \zeta\right) \\
& M_{21}^{\infty}(\zeta)=\frac{\alpha_{0}}{k_{1}} e^{-\frac{\pi}{4} \nu\left(z_{0}\right)}\left[\partial_{\zeta}\left(D_{a}\left(e^{\frac{i \pi}{4}} \zeta\right)\right)+\frac{i \zeta}{2} D_{a}\left(e^{\frac{i \pi}{4}} \zeta\right)\right] \\
& M_{12}^{\infty}(\zeta)=\frac{\alpha_{0}^{-1}}{k_{2}} e^{\frac{3 \pi}{4} \nu\left(z_{0}\right)}\left[\partial_{\zeta}\left(D_{-a}\left(e^{\frac{3 i \pi}{4}} \zeta\right)\right)-\frac{i \zeta}{2} D_{-a}\left(e^{\frac{3 i \pi}{4}} \zeta\right)\right], \\
& M_{22}^{\infty}(\zeta)=\alpha_{0}^{-1} e^{\frac{3 \pi}{4} \nu\left(z_{0}\right)} D_{-a}\left(e^{\frac{3 i \pi}{4}} \zeta\right) .
\end{aligned}
$$

Let $M_{\mathrm{II}}^{\infty}, M_{\mathrm{V}}^{\infty}$ be the analytic extensions to $\mathbb{C}$ of $\left.M^{\infty}\right|_{\mathrm{II}_{z_{0}}},\left.M^{\infty}\right|_{\mathrm{V}_{z_{0}}}$, respectively. Then, from the jump matrix $V^{\infty}$ we observe that for all $\zeta \in \mathbb{C}$

$$
M_{\mathrm{II}}^{\infty}(\zeta)=M_{\mathrm{V}}^{\infty}(\zeta)\left(\begin{array}{cc}
1 & r\left(z_{0}\right) \\
0 & 1
\end{array}\right)\left(\begin{array}{cc}
1 & 0 \\
r\left(z_{0}\right) & 1
\end{array}\right)=M_{\mathrm{V}}^{\infty}(\zeta)\left(\begin{array}{cc}
1+\frac{\left|r\left(z_{0}\right)\right|^{2}}{r\left(z_{0}\right)} & r\left(z_{0}\right) \\
1
\end{array}\right) .
$$

Therefore, as $\operatorname{det} M_{V}^{\infty}=1$,

$$
\begin{aligned}
\overline{r\left(z_{0}\right)} & =\left(M_{\mathrm{II}}^{\infty}\right)_{21}\left(M_{\mathrm{V}}^{\infty}\right)_{11}-\left(M_{\mathrm{II}}^{\infty}\right)_{11}\left(M_{\mathrm{V}}^{\infty}\right)_{21} \\
& =\frac{\alpha_{0}^{2}}{k_{1}} e^{\frac{\pi}{2} \nu\left(z_{0}\right)} W\left[D_{a}\left(e^{\frac{i \pi}{4}} \zeta\right), D_{a}\left(e^{\frac{-3 i \pi}{4}} \zeta\right)\right] \\
& =\frac{\alpha_{0}^{2}}{k_{1}} e^{\frac{\pi}{2} \nu\left(z_{0}\right)}\left[\frac{\sqrt{2 \pi}}{\Gamma(-a)} e^{\frac{i \pi}{4}}\right]
\end{aligned}
$$

where $W(f, g)=f g^{\prime}-f^{\prime} g$ is the Wronskian of $f$ and $g$. Hence,

$$
k_{1}=\frac{\sqrt{2 \pi} e^{\frac{i \pi}{4}} \alpha_{0}^{2} e^{\frac{\pi}{2} \nu\left(z_{0}\right)}}{\overline{r\left(z_{0}\right)} \Gamma(-a)}=\beta\left(z_{0}\right) e^{i\left[x^{2} /(2 t)+\nu\left(z_{0}\right) \log t\right]},
$$

where

$$
\begin{aligned}
\left|\beta\left(z_{0}\right)\right|^{2}= & \frac{2 \pi e^{\pi \nu\left(z_{0}\right)}}{\left|r\left(z_{0}\right)\right|^{2}\left|\Gamma\left(i \nu\left(z_{0}\right)\right)\right|^{2}}=\frac{2 \pi e^{\pi \nu\left(z_{0}\right)}}{\left|r\left(z_{0}\right)\right|^{2}}\left(\frac{\nu\left(z_{0}\right) \sinh \left(\pi \nu\left(z_{0}\right)\right)}{\pi}\right) \\
= & \nu\left(z_{0}\right) \\
\arg \beta\left(z_{0}\right)= & \frac{\pi}{4}-2 i\left(\beta\left(z_{0}, z_{0}\right)-i \nu\left(z_{0}\right)\right)+\arg \left(r\left(z_{0}\right)\right)-\arg \left(\Gamma\left(i \nu\left(z_{0}\right)\right)\right) \\
= & \frac{\pi}{4}+\frac{1}{\pi} \int_{-\infty}^{z_{0}} \log \left(z_{0}-s\right) \mathrm{d} \log \left(1+|r(s)|^{2}\right) \\
& \quad+\arg \left(r\left(z_{0}\right)\right)-\arg \left(\Gamma\left(i \nu\left(z_{0}\right)\right) .\right.
\end{aligned}
$$

and

$$
k_{2}=\frac{a}{i k_{1}}=-\frac{\nu\left(z_{0}\right)}{k_{1}}=-\overline{\beta\left(z_{0}\right)} e^{-i\left[x^{2} /(2 t)+\nu\left(z_{0}\right) \log t\right]} .
$$


Inserting (6.12), (6.14) into (6.9), 6.10) respectively, we obtain the explicit formulae for $M^{\infty}$ in region II. On the other regions, $M^{\infty}$ is obtained by simply using the constant jump matrix $V^{\infty}$.

\section{Proof of Theorem 1$]$}

For functions $u, \check{u} \in H^{1,1}(\mathbb{R})$, let $\psi^{ \pm}(x, z), \check{\psi}^{ \pm}(x, z)$ be the associated ZS-AKNS solutions, respectively (see Section 3). Set $m^{ \pm}=$ $\left(m_{1}^{ \pm}, m_{2}^{ \pm}\right) \equiv \psi^{ \pm} e^{-i x z \sigma}, \check{m}^{ \pm}=\left(\check{m}_{1}^{ \pm}, \check{m}_{2}^{ \pm}\right) \equiv \check{\psi}^{ \pm} e^{-i x z \sigma}$. Denote $\Delta u=$ $\check{u}-u, \Delta m_{1}^{+}=\check{m}_{1}^{+}-m_{1}^{+}$, etc. We write $m_{1}^{+}=\left(\left(m_{1}^{+}\right)_{1},\left(m_{1}^{+}\right)_{2}\right)^{T}$, etc.

Lemma 7.1. Let $u \in H^{1,1}(\mathbb{R})$. For any $\check{u} \in H^{1,1}(\mathbb{R})$ such that $\| \check{u}-$ $u \|_{H^{1,1}(\mathbb{R})} \leq \epsilon$,

$$
\left\|\Delta m_{1}^{+}(\cdot, z)\right\|_{L^{\infty}(\mathbb{R})} \leq c_{1}\|\Delta u\|_{H^{1,1}} \leq c_{1} \epsilon,
$$

where $c_{1}$ depends on $u$ and is uniform on $z \in \overline{\mathbb{C}^{+}}$. Moreover, for any fixed $z \in \mathbb{C}^{+}$,

$$
\left\|\Delta\left(m_{1}^{+}\right)_{2}(\cdot, z)\right\|_{H^{0,1}(\mathbb{R})} \leq c_{2} \epsilon, \quad\left\|\Delta \partial_{x} m_{1}^{+}(\cdot, z)\right\|_{L^{2}(\mathbb{R})} \leq c_{2} \epsilon
$$

where $c_{2}$ depends on $u$ and $z$. A similar result holds for $m_{2}^{-}(x, z)$.

Proof. The difference $\Delta m_{1}^{+}$solves the integral equation (cf. (3.11))

$$
\Delta m_{1}^{+}=\mathfrak{b}_{+}+T_{+}\left(\Delta m_{1}^{+}\right)
$$

where

$$
\begin{aligned}
\mathfrak{b}_{+} & =-\int_{x}^{\infty}\left(\begin{array}{cc}
1 & 0 \\
0 & e^{-i z(x-y)}
\end{array}\right)\left(\begin{array}{cc}
0 & \Delta u \\
-\Delta \bar{u} & 0
\end{array}\right) m_{1}^{+} \mathrm{d} y \\
& =\int_{x}^{\infty}\left(\begin{array}{c}
-\Delta u\left(m_{1}^{+}\right)_{2} \\
\Delta \bar{u} e^{-i z(x-y)}\left(m_{1}^{+}\right)_{1}
\end{array}\right) \mathrm{d} y .
\end{aligned}
$$

and $T_{+}$is the operator acting on column vectors $\mathfrak{f}$ defined by

$$
T_{+}(\mathfrak{f})(x)=-\int_{x}^{\infty}\left(\begin{array}{cc}
1 & 0 \\
0 & e^{-i z(x-y)}
\end{array}\right)\left(\begin{array}{cc}
0 & \check{u} \\
-\bar{u} & 0
\end{array}\right) \mathfrak{f}(y) \mathrm{d} y
$$

Since $\left(m_{1}^{+}\right)_{2} \in H^{1,1}(\mathbb{R}) \subset L^{1}(\mathbb{R})$ and $\left(m_{1}^{+}\right)_{1} \in L^{\infty}(\mathbb{R})$ (see Proposition 3.5),

$$
\left\|\mathfrak{b}_{+}\right\|_{L^{\infty}(\mathbb{R})} \leq c\|\Delta u\|_{H^{1,1}}
$$

uniformly on $z \in \overline{\mathbb{C}^{+}}$. For $n \geq 1$,

$$
\begin{aligned}
\left|T_{+}^{n}\left(\mathfrak{b}_{+}\right)\right| & \leq\left\|\mathfrak{b}_{+}\right\|_{\infty} \int_{x}^{\infty}\left|\check{u}\left(y_{1}\right)\right| \int_{y_{1}}^{\infty}\left|\check{u}\left(y_{2}\right)\right| \cdots \int_{y_{n-1}}^{\infty}\left|\check{u}\left(y_{n}\right)\right| \\
& \leq\left\|\mathfrak{b}_{+}\right\|_{\infty} \frac{1}{n !}\left[\int_{x}^{\infty}|\check{u}(y)| \mathrm{d} y\right]^{n+1} .
\end{aligned}
$$


and hence

$$
\left\|\Delta m_{1}^{+}\right\|_{L^{\infty}(\mathbb{R})} \leq e^{\int_{-\infty}^{\infty}|\check{u}(y)| \mathrm{d} y}\left\|\mathfrak{b}_{+}\right\|_{\infty} \leq c\|\Delta u\|_{H^{1,1}} .
$$

Now we fix $z \in \mathbb{C}^{+}$. By Lemma 3.6.

$$
\left\|\left(\mathfrak{b}_{+}\right)_{2}\right\|_{H^{1,1}(\mathbb{R})} \leq c\left\|\Delta u\left(m_{1}^{+}\right)_{1}\right\|_{H^{0,1}} \leq c\|\Delta u\|_{H^{0,1}} .
$$

Assembling these results, we obtain

$$
\begin{aligned}
\left\|\Delta\left(m_{1}^{+}\right)_{2}\right\|_{H^{0,1}(\mathbb{R})} & =\left\|\left(\mathfrak{b}_{+}\right)_{2}+\left(T_{+}\left(\Delta m_{1}^{+}\right)\right)_{2}\right\|_{H^{0,1}} \\
& \leq c\|\Delta u\|_{H^{0,1}}+c\|\check{u}\|_{H^{0,1}}\left\|\Delta\left(m_{1}^{+}\right)_{1}\right\|_{L^{\infty}} \leq c\|\Delta u\|_{H^{1,1}} .
\end{aligned}
$$

For $\Delta \partial_{x} m_{1}^{+}$, since $\Delta \partial_{x} m_{1}^{+}=\left(\begin{array}{cc}0 & 0 \\ 0 & -i z\end{array}\right) \Delta m_{1}^{+}+\Delta Q m_{1}^{+}+\check{Q} \Delta m_{1}^{+}$, we see that

$$
\begin{aligned}
\left\|\Delta \partial_{x} m_{1}^{+}\right\|_{L^{2}(\mathbb{R})} & \leq c\left\|\Delta\left(m_{1}^{+}\right)_{2}\right\|_{L^{2}}+\|\Delta Q\|_{L^{2}}\left\|m_{1}^{+}\right\|_{L^{\infty}}+\|\check{Q}\|_{L^{2}}\left\|\Delta m_{1}^{+}\right\|_{L^{\infty}} \\
& \leq c\|\Delta u\|_{H^{1,1}} .
\end{aligned}
$$

and hence the proof is done.

Lemma 7.2 (cf. DZ03, Theorem 3.2). Let $u(x) \in H^{0,1}(\mathbb{R})$. Fix $x \in$ $\mathbb{R}$. Then, the associated $Z S-A K N S$ solutions $m^{ \pm}(x, \cdot) \in I+H^{1,0}(d z)$. Moreover, if $\|\check{u}-u\|_{H^{0,1}}<\epsilon$, then $\left\|\check{m}^{ \pm}(x, \cdot)-m^{ \pm}(x, \cdot)\right\|_{H^{1,0}} \leq c \epsilon$.

Proof. We will provide the proof only for $m^{+}$. Define operators $K_{u}$ acting on $2 \times 2$ matrix functions $B$ as follows,

$$
\left(K_{u} B\right)(x, z)=\int_{+\infty}^{x} e^{i(x-y) z \text { ad } \sigma} Q(y) B(y, z) d y, x, z \in \mathbb{R}
$$

Then, ZS-AKNS solutions for real $z$ satisfy $m^{+}=I+K_{u} m^{+}$. We use the following notation. If $\mathfrak{M}$ is a measure space and $\mathfrak{B}$ is a Banach space, then $\mathfrak{B} \otimes L^{p}(\mathfrak{M}) \equiv L^{p}(\mathfrak{M} \rightarrow \mathfrak{B})$ denotes the space of $\mathfrak{B}$-valued $L^{p}$ functions with norm $\|f\|_{\mathfrak{B} \otimes L^{p}(\mathfrak{M})}=\|\| f\left\|_{\mathfrak{B}}\right\|_{L^{p}(\mathfrak{M})}$. Direct calculations show that

$$
\begin{gathered}
\left\|K_{u} B\right\|_{L^{2}(d z) \otimes L^{\infty}(d x)} \leq c\|u\|_{L^{1}}\|B\|_{L^{2}(d z) \otimes L^{\infty}(d x)}, \\
\left\|K_{u} B\right\|_{L^{2}(d z) \otimes L_{\mathbb{R}^{+}}^{2}(d x)} \leq c\|u\|_{H^{0,1}}\|B\|_{L^{2}(d z) \otimes L^{\infty}(d x)},
\end{gathered}
$$

and

$$
\left\|K_{u} B\right\|_{L^{2}(d z) \otimes L^{\infty}(d x)} \leq c\|u\|_{L^{2}}\|B\|_{L^{2}(d z) \otimes L_{\mathbb{R}^{+}}^{2}(d x)},
$$

Standard iterations for the Volterra integral equation give

$$
\left\|\left(1-K_{u}\right)^{-1}\right\|_{L^{2}(d z) \otimes L^{\infty}(d x) \rightarrow L^{2}(d z) \otimes L^{\infty}(d x)} \leq c e^{\|u\|_{L^{1}}} .
$$


By Fourier theory and Hardy's inequality,

$$
\begin{aligned}
& \left\|K_{u} I\right\|_{L^{2}(d z) \otimes L^{\infty}(d x)}=c\left\|\left(\int_{+\infty}^{\langle x\rangle}|u|^{2} d y\right)^{\frac{1}{2}}\right\|_{L^{\infty}(d x)} \leq c\|u\|_{L^{2}} \\
& \left\|K_{u} I\right\|_{L^{2}(d z) \otimes L_{\mathbb{R}^{+}}^{2}(d x)}=c\left\|\left(\int_{+\infty}^{\langle x\rangle}|u|^{2} d y\right)^{\frac{1}{2}}\right\|_{L_{\mathbb{R}^{+}}^{2}(d x)} \leq c\|u\|_{H^{0,1}} .
\end{aligned}
$$

As $m^{+}=I+\left(1-K_{u}\right)^{-1} K_{u} I$, by using (7.3) we see that

$$
\left\|m^{+}-I\right\|_{L^{2}(d z) \otimes L^{\infty}(d x)} \leq c\left\|K_{u} I\right\|_{L^{2}(d z) \otimes L^{\infty}(d x)} \leq c,
$$

and hence by (7.4),

$$
\left\|m^{+}-I\right\|_{L^{2}(d z) \otimes L_{\mathbb{R}^{+}}^{2}(d x)}=\left\|K_{u}\left(m^{+}-I\right)+K_{u} I\right\|_{L^{2}(d z) \otimes L_{\mathbb{R}^{+}}^{2}(d x)} \leq c .
$$

Define $M \equiv\left(\partial_{z}-i x\right.$ ad $\left.\sigma\right) m^{+}$. Then $M$ satisfies the equation

$$
\begin{aligned}
M(x, z)= & -i \int_{+\infty}^{x} e^{i(x-y) z \operatorname{ad} \sigma} \operatorname{ad} \sigma(y Q(y)) m^{+}(y, z) d y \\
& +\int_{+\infty}^{x} e^{i(x-y) z \text { ad } \sigma} Q(y) M(y, z) d y \\
= & -i K_{\cdot u(\cdot)}^{\prime} m^{+}+K_{u} M
\end{aligned}
$$

where $K^{\prime}$ is the operator defined in (7.2) with ad $\sigma(Q)$ in place of $Q$. As $M=-i\left(1-K_{u}\right)^{-1} K_{\cdot \Delta u(\cdot)}^{\prime} m^{+}$, it follows by (7.5) that

$$
\begin{aligned}
\|M\|_{L^{2}(d z) \otimes L^{\infty}(d x)} & \leq c\left\|K_{\cdot u(\cdot)}^{\prime}\left(m^{+}-I\right)+K_{\cdot u(\cdot)}^{\prime} I\right\|_{L^{2}(d z) \otimes L^{\infty}(d x)} \\
& \leq c\|\cdot u(\cdot)\|_{L^{2}}\left(\left\|m^{+}-I\right\|_{L^{2}(d z) \otimes L_{\mathbb{R}^{+}}^{2}(d x)}+1\right) \leq c .
\end{aligned}
$$

The equations for $\Delta m^{+}=\check{m}^{+}-m^{+}, \Delta M=\check{M}-M$ are given by (cf. (7.1))

$$
\begin{aligned}
& \Delta m^{+}=K_{\Delta u} \check{m}^{+}+K_{u} \Delta m^{+}, \\
& \Delta M=-i\left(K_{\cdot \Delta u(\cdot)}^{\prime} \check{m}^{+}+K_{\cdot u(\cdot)}^{\prime} \Delta m^{+}\right)+K_{\Delta u} \check{M}+K_{u} \Delta M .
\end{aligned}
$$

Similarly, we have

$$
\begin{aligned}
\left\|\Delta m^{+}\right\|_{L^{2}(d z) \otimes L^{\infty}(d x)} & \leq c\left\|K_{\Delta u}\left(\check{m}^{+}-I\right)+K_{\Delta u} I\right\|_{L^{2}(d z) \otimes L^{\infty}(d x)} \\
& \leq c\|\Delta u\|_{H^{0,1}}
\end{aligned}
$$


and hence

$$
\begin{aligned}
\left\|\Delta m^{+}\right\|_{L^{2}(d z) \otimes L_{\mathbb{R}^{+}}^{2}(d x)=} & \left\|K_{\Delta u}\left(\check{m}^{+}-I\right)+K_{\Delta u} I+K_{u} \Delta m^{+}\right\|_{L^{2}(d z) \otimes L_{\mathbb{R}^{+}}^{2}(d x)} \\
\leq & c\|\Delta u\|_{H^{0,1}}\left(\left\|\check{m}^{+}-I\right\|_{L^{2}(d z) \otimes L^{\infty}(d x)}+1\right) \\
& \quad+c\|u\|_{H^{0,1}}\left\|\Delta m^{+}\right\|_{L^{2}(d z) \otimes L^{\infty}(d x)} \\
\leq & c\|\Delta u\|_{H^{0,1}}
\end{aligned}
$$

Therefore for $\Delta M$ we see that

$$
\begin{aligned}
\|\Delta M\|_{L^{2}(d z) \otimes L^{\infty}(d x) \leq} \leq c\left\|K_{\cdot \Delta u(\cdot)}^{\prime}\left(\check{m}^{+}-I\right)+K_{\cdot \Delta u(\cdot)}^{\prime} I\right\|_{L^{2}(d z) \otimes L^{\infty}(d x)} & \\
& \quad+c\left\|-i K_{\cdot u(\cdot)}^{\prime} \Delta m^{+}+K_{\Delta u} \check{M}\right\|_{L^{2}(d z) \otimes L^{\infty}(d x)} \\
\leq & c\|\Delta u\|_{H^{0,1}}
\end{aligned}
$$

In particular, $\partial_{z} m^{+}(0, z)=M(0, z) \in L^{2}(\mathbb{R})$. Also, $\partial_{z} \Delta m^{+}(0, z)=$ $\Delta M(0, z) \in L^{2}(\mathbb{R})$ and hence $\left\|\Delta m^{+}\right\|_{H^{1,0}} \leq c\|\Delta u\|_{H^{0,1}}$.

Let $\psi_{1}^{+},\left(\psi_{0}\right)_{1}^{+}$be the first columns of the ZS-AKNS solutions for $u_{0}^{e}, \eta_{\mu_{0}}$ in Theorem 1 and (4.50), respectively. Set $m_{1}^{+} \equiv \psi_{1}^{+} e^{-i x z / 2}$, $\left(m_{0}\right)_{1}^{+} \equiv\left(\psi_{0}\right)_{1}^{+} e^{-i x z / 2}(x \geq 0)$, respectively. By Remark 4.33, $\eta_{\mu_{0}}(x)$ in (4.50) is the Bäcklund extension of $v_{\mu_{0}}(x)$ and it is straightforward to see that $\left(m_{0}\right)_{1}^{+}$and $\left(m_{0}\right)_{2}^{-}$have the explicit formulae (see [RS02])

$$
\begin{aligned}
& \left(m_{0}\right)_{1}^{+}(x, z)=\frac{1}{z+i \mu_{0}}\left(\begin{array}{c}
z+i \mu_{0} \tanh \left(\mu_{0} x+\tanh ^{-1}\left(q / \mu_{0}\right)\right) \\
i \mu_{0} \operatorname{sech}\left(\mu_{0} x+\tanh ^{-1}\left(q / \mu_{0}\right)\right)
\end{array}\right), \\
& \left(m_{0}\right)_{2}^{-}(x, z)=\frac{1}{z+i \mu_{0}}\left(\begin{array}{c}
i \mu_{0} \operatorname{sech}\left(\mu_{0} x+\tanh ^{-1}\left(q / \mu_{0}\right)\right) \\
z-i \mu_{0} \tanh \left(\mu_{0} x+\tanh ^{-1}\left(q / \mu_{0}\right)\right)
\end{array}\right) .
\end{aligned}
$$

Define (cf. Proposition 4.35)

$$
\begin{aligned}
& \left(\begin{array}{l}
A(z) \\
B(z)
\end{array}\right) \equiv m_{1}^{+}(0, z), \quad\left(\begin{array}{l}
A_{0}(z) \\
B_{0}(z)
\end{array}\right) \equiv\left(m_{0}\right)_{1}^{+}(0, z), \\
& A_{1}(z) \equiv A(z)-A_{0}(z), \quad B_{1}(z) \equiv B(z)-B_{0}(z) .
\end{aligned}
$$

Then

$$
\left(\begin{array}{l}
A_{0}(z) \\
B_{0}(z)
\end{array}\right)=\frac{1}{z+i \mu_{0}}\left(\begin{array}{c}
z+i q \\
i \sqrt{\mu_{0}^{2}-q^{2}}
\end{array}\right) .
$$

By Proposition 4.35, the scattering data for the Bäcklund extension $u_{0}^{e}$ of $\left.u_{0}(x)\right|_{\mathbb{R}^{+}}=\left.u(x, 0)\right|_{\mathbb{R}^{+}}$is given by

$$
\begin{aligned}
& a(z)=\frac{1}{z-i \beta}\left[(z+i q) \frac{z-i \mu_{0}}{z+i \mu_{0}}+(z-i q) g_{1}(z)-(z+i q) g_{2}(z)\right], \\
& b(z)=\frac{1}{z+i \beta}\left[(z+i q) g_{3}(z)+(z-i q) g_{3}(-z)\right] .
\end{aligned}
$$


where

$$
\begin{aligned}
& g_{1}(z)=A_{0}(z) \overline{A_{1}(-\bar{z})}+A_{1}(z) \overline{A_{0}(-\bar{z})}+A_{1}(z) \overline{A_{1}(-\bar{z})} \\
& g_{2}(z)=B_{0}(z) \overline{B_{1}(-\bar{z})}+B_{1}(z) \overline{B_{0}(-\bar{z})}+B_{1}(z) \overline{B_{1}(-\bar{z})} \\
& g_{3}(z)=A_{0}(-z) B_{1}(z)+A_{1}(-z) B_{0}(z)+A_{1}(-z) B_{1}(z)
\end{aligned}
$$

and $\beta$ is determined as in (4.51). By Lemma 7.2 , we have

$$
\left\|A_{1}\right\|_{H^{1,0}(\mathbb{R})} \leq c \epsilon, \quad\left\|B_{1}\right\|_{H^{1,0}(\mathbb{R})} \leq c \epsilon,
$$

and hence

$$
\left\|g_{j}\right\|_{H^{1,0}(\mathbb{R})} \leq c \epsilon, \quad 1 \leq j \leq 3
$$

Thus,

$$
\|b\|_{L^{2}(\mathbb{R})} \leq c \epsilon,\|b\|_{L^{\infty}(\mathbb{R})} \leq c \epsilon \text { and }\|b\|_{H^{1,0}(\mathbb{R})} \leq c \epsilon|q|^{-\frac{1}{2}} .
$$

Define $a_{0}(z) \equiv \frac{z+i q}{z-i \beta} \frac{z-i \mu_{0}}{z+i \mu_{0}}$. Then,

$$
\left\|a-a_{0}\right\|_{L^{\infty}(\mathbb{R})} \leq c \epsilon, \quad\left\|a-a_{0}\right\|_{H^{1,0}(\mathbb{R})} \leq c \epsilon|q|^{-\frac{1}{2}} .
$$

As $a\left(z ; \eta_{\mu_{0}}\right)=\frac{z-i \mu_{0}}{z+i \mu_{0}}, \eta_{\mu_{0}}$ is generic and it follows from the proof of Proposition 4.38 that for all sufficiently small $\epsilon>0 a(z)=a\left(z ; u_{0}^{e}\right)$ is also generic. As $a\left(z ; \eta_{\mu_{0}}\right)$ has only one zero in $\mathbb{C}^{+}$, it follows that $\beta\left(\eta_{\mu_{0}}\right)=(-1)^{1} q=-q$. Suppose $q>0$. Then $a_{1}\left(z ; \eta_{\mu_{0}}\right) \equiv a\left(z ; \eta_{\mu_{0}}\right)(z-$ $\left.i \beta\left(\eta_{\mu_{0}}\right)\right)=\frac{z-i \mu_{0}}{z+i \mu_{0}}(z+i q)$ has only one zero in $\mathbb{C}^{+}$, and hence, $a_{1}\left(z ; u_{0}^{e}\right)$ also has only one zero $z_{1} \sim i \mu_{0}$ in $\mathbb{C}^{+}$, by Rouché's theorem. But

$$
a_{1}\left(z ; u_{0}^{e}\right)=a\left(z ; u_{0}^{e}\right)\left(z-i \beta\left(u_{0}^{e}\right)\right),
$$

and as $\left|\beta\left(u_{0}^{e}\right)\right|=|q|<\mu_{0}$, we see that $a\left(z_{1} ; u_{0}^{e}\right)=0$. Hence $a\left(z ; u_{0}^{e}\right)$ also has one zero $z_{1} \sim i \mu_{0}$ in $\mathbb{C}^{+}$and necessarily $\beta\left(u_{0}^{e}\right)=(-1)^{1} q=-q$, which is consistent with the fact that $a_{1}\left(i q ; u_{0}^{e}\right) \neq 0$ (see (7.15)). Now suppose $q<0$. Then $a_{1}\left(z ; \eta_{\mu_{0}}\right)$ has two zeros $i \mu_{0},-i q$. Again by Rouché, $a_{1}\left(z ; u_{0}^{e}\right)$ has two zeros $z_{1} \sim i \mu_{0}, z_{2} \sim-i q$ in $\mathbb{C}^{+}$. If $z_{2}=-i q$, then as $a\left(-i q ; u_{0}^{e}\right) \neq 0$ by Lemma 4.22, it follows from (7.15) that $\beta\left(u_{0}^{e}\right)=-q$, and that $a\left(z ; u_{0}^{e}\right)=\frac{a_{1}\left(z ; u_{0}^{e}\right)}{z+i q}$ has only one zero $z_{1} \sim i \mu_{0}$ in $\mathbb{C}^{+}$(note that this is consistent with $\beta\left(u_{0}^{e}\right)=-q$ ). On the other hand if $z_{2} \neq-i q$, then it follows from (7.15) that $\beta\left(u_{0}^{e}\right)=q$ (otherwise $a_{1}\left(z ; u_{0}^{e}\right)$ would have three zeros in $\left.\mathbb{C}^{+}\right)$. But then, again from (7.15), we see that $a\left(z ; u_{0}^{e}\right)$ has two zeros $z_{1} \sim i \mu_{0}, z_{2} \sim-i q$ in $\mathbb{C}^{+}$(note again that this is consistent with $\left.\beta\left(u_{0}^{e}\right)=q\right)$. By the symmetry condition $a\left(z ; u_{0}^{e}\right)=\overline{a\left(-\bar{z} ; u_{0}^{e}\right)}$ from (4.39), we see that the zeros $z_{1}, z_{2}$ must lie on $i \mathbb{R}^{+}$. We have proved the following result.

Proposition 7.3 (Zeros of $a\left(z ; u_{0}^{e}\right)$ in $\left.\mathbb{C}^{+}\right)$.

(i) If $q>0, a\left(z ; u_{0}^{e}\right)$ has one simple zero $z_{1}=i \mu_{1} \in i \mathbb{R}^{+}, \mu_{1} \sim \mu_{0}$. 
(ii) If $q<0$, and $a_{1}\left(-i q ; u_{0}^{e}\right)=0$, then $a\left(z ; u_{0}^{e}\right)$ has one simple zero $z_{1}=i \mu_{1} \in i \mathbb{R}^{+}, \mu_{1} \sim \mu_{0}$.

(iii) If $q<0$, and $a_{1}\left(-i q ; u_{0}^{e}\right) \neq 0$, then $a\left(z ; u_{0}^{e}\right)$ has two simple zeros $z_{1}=i \mu_{1} \in i \mathbb{R}^{+}, \mu_{1} \sim \mu_{0}$ and $z_{2}=i \mu_{2} \in i \mathbb{R}^{+}, \mu_{2} \sim-q$, $\mu_{2} \neq-q$.

Lemma 7.4. In the notation of Proposition 7.3,

$$
\begin{aligned}
& \mu_{1}=\mu_{0}+\epsilon w_{1}+O\left(\epsilon q+\epsilon^{2}\right), \\
& \mu_{2}=-q+O\left(\epsilon^{2} q\right)
\end{aligned}
$$

where $w_{1}=\int_{\mathbb{R}} \operatorname{Re} w(y) v_{\mu_{0}}(y) \mathrm{d} y$. Moreover, $\sqrt{\frac{\mu_{2}+q}{\mu_{2}-q}}=O(\epsilon)$.

Proof. For $z_{2}=i \mu_{2}$, as $\beta=+q$ and $\mu_{2} \neq-q$ by Proposition 7.3 (iii), we have from (7.9),

$$
0=a\left(z_{2}\right)=\frac{\mu_{2}+q}{\mu_{2}-q} \frac{\mu_{2}-\mu_{0}}{\mu_{2}+\mu_{0}}+g_{1}\left(z_{2}\right)-\frac{\mu_{2}+q}{\mu_{1}-q} g_{2}\left(z_{2}\right) .
$$

Set $K=\frac{\mu_{2}+q}{\mu_{2}-q}$. From (7.12) and (7.16) we see that $K=-g_{1}\left(z_{2}\right)\left(\frac{\mu_{2}-\mu_{0}}{\mu_{2}+\mu_{0}}-\right.$ $\left.g_{2}\left(z_{2}\right)\right)^{-1}=O(\epsilon)$. Hence $\mu_{2}=-q \frac{1+K}{1-K}=-q+O(\epsilon q)$, which implies that $A_{0}\left(z_{2}\right)=\frac{\mu_{2}+q}{\mu_{2}+\mu_{0}}=O(\epsilon q)$. But then from (7.10) $g_{1}\left(z_{2}\right)=$ $A_{0}\left(z_{2}\right)\left(A_{1}\left(z_{2}\right)+\overline{A_{1}\left(z_{2}\right)}\right)+O\left(\epsilon^{2}\right)=O\left(\epsilon^{2}\right)$, which now implies that $K=O\left(\epsilon^{2}\right)$ and hence $\mu_{2}=-q+O\left(\epsilon^{2} q\right)$.

For $z_{1}=i \mu_{1}$, let $\left(\psi_{1}^{+}, \psi_{2}^{-}\right),\left(\left(\psi_{0}\right)_{1}^{+},\left(\psi_{0}\right)_{2}^{-}\right)$be the ZS-AKNS solutions for $u_{0}^{e}, \eta_{\mu_{0}}$ in $\overline{\mathbb{C}^{+}}$, respectively. Define $\varphi(x, z) \equiv \dot{\psi}_{1}^{+}-\gamma_{1} \dot{\psi}_{2}^{-}, z \in \mathbb{C}^{+}$ where $\dot{\psi}_{1}^{+}=\partial_{z} \psi_{1}^{+}$and $\dot{\psi}_{2}^{-}=\partial_{z} \psi_{2}^{-}$and $\gamma_{1}$ is the norming constant for $z_{1}$. Fix $z=z_{1}=i \mu_{1}$ and set $\phi(x)=\left(\psi_{1}^{+}, \varphi\right)\left(x, z_{1}\right)$. As

$$
\begin{aligned}
0 \neq a^{\prime}\left(z_{1}\right) & =\operatorname{det}\left(\dot{\psi}_{1}^{+}, \psi_{2}^{-}\right)+\operatorname{det}\left(\psi_{1}^{+}, \dot{\psi}_{2}^{-}\right) \\
& =\operatorname{det}\left(\dot{\psi}_{1}^{+}, \gamma_{1}^{-1} \psi_{1}^{+}\right)+\operatorname{det}\left(\psi_{1}^{+}, \dot{\psi}_{2}^{-}\right) \\
& =-\gamma_{1}^{-1} \operatorname{det} \phi,
\end{aligned}
$$

$\phi^{-1}$ exists for all $x \in \mathbb{R}$. A simple computation shows that $\phi_{x}=$ $\left(i z_{1} \sigma+Q\right) \phi$ where $Q=\left(\begin{array}{cc}0 & u_{0}^{e} \\ -\overline{u_{0}^{e}} & 0\end{array}\right)$. As $\left(\left(\psi_{0}\right)_{1}^{+}\right)_{x}=\left(i z_{1} \sigma+Q_{0}\right)\left(\psi_{0}\right)_{1}^{+}$ where $Q_{0}=\left(\begin{array}{cc}0 & \eta_{\mu_{0}} \\ -\bar{\eta}_{\mu_{0}} & 0\end{array}\right)$, we see that

$$
\left(\phi^{-1}\left(\psi_{0}\right)_{1}^{+}\right)_{x}=-\left(\phi^{-1} \Delta Q \phi\right)\left(\phi^{-1}\left(\psi_{0}\right)_{1}^{+}\right), \quad \Delta Q=Q-Q_{0} .
$$

Let $M=\phi e^{-i x z_{1} \sigma}$ and $\left(m_{0}\right)_{1}^{+}=\left(\psi_{0}\right)_{1}^{+} e^{-i x z_{1} / 2}$. Then,

$$
\phi^{-1}\left(\psi_{0}\right)_{1}^{+}=\left(\begin{array}{cc}
1 & 0 \\
0 & e^{i x z_{1}}
\end{array}\right) M^{-1}\left(m_{0}\right)_{1}^{+} .
$$


Since $\left(m_{0}\right)_{1}^{+} \rightarrow e_{1}$ and $M \rightarrow\left(\begin{array}{cc}1 & 0 \\ 0 & -\gamma_{1} a^{\prime}\left(z_{1}\right)\end{array}\right)$ as $x \rightarrow+\infty$, it follows from (7.18) that

$$
\phi^{-1}\left(\psi_{0}\right)_{1}^{+}=e_{1}+\int_{x}^{\infty} \phi^{-1} \Delta Q \phi\left(\phi^{-1}\left(\psi_{0}\right)_{1}^{+}\right)
$$

and hence

$$
M^{-1}\left(m_{0}\right)_{1}^{+}=e_{1}+\int_{x}^{\infty}\left(\begin{array}{cc}
1 & 0 \\
0 & e^{-i(x-y) z_{1}}
\end{array}\right) G M^{-1}\left(m_{0}\right)_{1}^{+} \mathrm{d} y,
$$

where $G=M^{-1} \Delta Q M$. As $M(x)$ is uniformly bounded on $\mathbb{R}$ and $\|\Delta Q\|_{H^{0,1}} \leq c \epsilon$, it follows by a standard iteration that

$$
M^{-1}\left(m_{0}\right)_{1}^{+}=e_{1}+\int_{x}^{\infty}\left(\begin{array}{cc}
1 & 0 \\
0 & e^{-i(x-y) z_{1}}
\end{array}\right) G e_{1} \mathrm{~d} y+O\left(\epsilon^{2}\right),
$$

uniformly on $x \geq 0$. In particular, multiplying by $M$ and setting $x=0$, we obtain

$$
\left(\begin{array}{l}
A_{1}\left(z_{1}\right) \\
B_{1}\left(z_{1}\right)
\end{array}\right)=m_{1}^{+}\left(0, z_{1}\right)-\left(m_{0}\right)_{1}^{+}\left(0, z_{1}\right)=-M(0)\left(\begin{array}{l}
f_{1} \\
f_{2}
\end{array}\right)+O\left(\epsilon^{2}\right)
$$

where $f_{1}=\int_{0}^{\infty} G_{11}$ and $f_{2}=\int_{0}^{\infty} e^{-\mu_{1} y} G_{21}$. Set

$$
M_{0} \equiv\left(\left(\psi_{0}\right)_{1}^{+},\left(\dot{\psi}_{0}\right)_{1}^{+}-\gamma_{1}\left(\dot{\psi}_{0}\right)_{2}^{-}\right) e^{-i x z_{1} \sigma} .
$$

As $A_{0}\left(z_{1}\right) \neq 0$ by (7.8), it follows from (4.54) and (7.19) that the norming constant $\gamma_{1}=\gamma\left(z_{1}\right)$ for $u_{0}^{e}$ is given by

$$
\gamma_{1}=\frac{z_{1}-i \beta}{z_{1}+i q} \frac{A\left(z_{1}\right)}{B\left(z_{1}\right)}=(1+O(\epsilon+q)) \frac{A_{0}\left(z_{1}\right)}{B_{0}\left(z_{1}\right)}=1+O(\epsilon+q) .
$$

From (7.9) and (7.12), we have

$$
0=a\left(z_{1}\right)=\frac{\mu_{1}+q}{\mu_{1}-\beta} \frac{\mu_{1}-\mu_{0}}{\mu_{1}+\mu_{0}}+g_{1}\left(z_{1}\right)-g_{2}\left(z_{1}\right)+O(\epsilon q),
$$

and hence, again by (7.12),

$$
\mu_{1}=\mu_{0}+O(q+\epsilon) .
$$

As $\left(\begin{array}{l}A_{0}\left(z_{1}\right) \\ B_{0}\left(z_{1}\right)\end{array}\right)=\frac{1}{2}\left(\begin{array}{l}1 \\ 1\end{array}\right)+O(\epsilon)$ by (7.8), it follows from (7.10) and (7.11) that

$$
g_{1}\left(z_{1}\right)-g_{2}\left(z_{1}\right)=\operatorname{Re}\left(A_{1}\left(z_{1}\right)-B_{1}\left(z_{1}\right)\right)+O\left(\epsilon q+\epsilon^{2}\right)
$$

Direct calculation using (77.6) and (7.22) shows that $M_{0}\left(0, z_{1}\right)=\frac{1}{2}\left(\begin{array}{cc}1 & -i \mu_{0}^{-1} \\ 1 & i \mu_{0}^{-1}\end{array}\right)+$ $O(q+\epsilon)$. By Lemma 7.1, $\left|m_{1}^{+}(0, z)-\left(m_{0}\right)_{1}^{+}(0, z)\right| \leq c \epsilon$ and $\mid m_{2}^{-}(0, z)-$ $\left(m_{0}\right)_{2}^{-}(0, z) \mid \leq c \epsilon$ for $z \in \overline{\mathbb{C}^{+}}$. Then it follows by analyticity of $m_{1}^{+}(0, z)$, etc. that

$$
\left|M\left(0, z_{1}\right)-\left(M_{0}\right)\left(0, z_{1}\right)\right| \leq c \epsilon
$$


Hence from (7.19) and (7.20),

$$
\begin{aligned}
A_{1}\left(z_{1}\right)-B_{1}\left(z_{1}\right) & =-(1,-1) M(0)\left(\begin{array}{l}
f_{1} \\
f_{2}
\end{array}\right)+O\left(\epsilon^{2}\right) \\
& =-(1,-1) M_{0}(0)\left(\begin{array}{l}
f_{1} \\
f_{2}
\end{array}\right)+O\left(\epsilon^{2}\right) \\
& =\frac{i f_{2}}{\mu_{0}}+O\left(\epsilon q+\epsilon^{2}\right) .
\end{aligned}
$$

Now, from (3.24), $a^{\prime}\left(z_{1}\right)=\frac{1}{z_{1}-\overline{z_{1}}} e^{-l\left(z_{1}\right)}$ if $z_{1}$ is the only (simple) zero of $a(z)$ in $\mathbb{C}^{+}$and $a^{\prime}\left(z_{1}\right)=\frac{1}{z_{1}-\overline{z_{1}}} \frac{z_{1}-z_{2}}{z_{1}-\bar{z}_{2}} e^{-l\left(z_{1}\right)}=\frac{1}{z_{1}-\overline{z_{1}}} e^{-l\left(z_{1}\right)}+O(q)$ if $a(z)$ has a second (simple) zero at $i \mu_{2} \sim O(q)$. As $l\left(z_{1}\right)=O\left(\|r\|_{L^{2}}^{2}\right)=O\left(\epsilon^{2}\right)$, it follows from (7.17) that $\operatorname{det} M=\operatorname{det} \phi=-\gamma_{1} a^{\prime}\left(z_{1}\right)=-\frac{1}{2 i \mu_{1}}+O(q+\epsilon)$. Assembling the above results,

$$
\begin{aligned}
\operatorname{Re}\left(i f_{2}\right) & =\operatorname{Re} \int_{0}^{\infty} i e^{-\mu_{1} y}\left(M^{-1} \Delta Q M\right)_{21} \mathrm{~d} y \\
& =\operatorname{Re} \int_{0}^{\infty} \frac{i e^{-\mu_{1} y}}{\operatorname{det} M}\left(-\epsilon w M_{21}^{2}-\epsilon \bar{w} M_{11}^{2}\right) \mathrm{d} y \\
& =\operatorname{Re} \int_{0}^{\infty} \frac{i e^{-\mu_{1} y}}{\operatorname{det} M}\left(-\epsilon w\left(M_{0}\right)_{21}^{2}-\epsilon \bar{w}\left(M_{0}\right)_{11}^{2}\right) \mathrm{d} y+O\left(\epsilon^{2}\right) \\
& =-\epsilon \int_{0}^{\infty} \operatorname{Rew}(y) v_{\mu_{0}}(y) \mathrm{d} y+O\left(\epsilon q+\epsilon^{2}\right) \\
& =-\frac{\epsilon w_{1}}{2}+O\left(\epsilon q+\epsilon^{2}\right)
\end{aligned}
$$

Thus it follows from (7.21), (7.23) and (7.25) that $\mu_{1}=\mu_{0}+\epsilon w_{1}+$ $O\left(\epsilon q+\epsilon^{2}\right)$.

Remark 7.5. The zeros of $a(z)$ can be written in terms of $r(z)$ and $w^{e} \equiv u_{0}^{e}-\eta_{\mu_{0}}$ using the conserved integrals for NLS. Recall that the conserved integrals can be read off from the expansion of $\log a(z)=$ $\sum_{n=1}^{\infty} I_{n} z^{-n}$ as $z \rightarrow \infty$ (see [ZS72]). The first 3 coefficients in the expansion are given by

$$
\begin{aligned}
& I_{1}=-i \int_{\mathbb{R}}|u(x, t)|^{2} \mathrm{~d} x, \quad I_{2}=\frac{1}{2} \int_{\mathbb{R}}\left(u \bar{u}_{x}-u_{x} \bar{u}\right)(x, t) \mathrm{d} x, \\
& I_{3}=i \int_{\mathbb{R}}\left(|u|^{4}-\left|u_{x}\right|^{2}\right)(x, t) \mathrm{d} x .
\end{aligned}
$$


Also, if $a(z)$ has one zero, it follows from (3.24) that as $z \rightarrow \infty, \operatorname{Im} z>$ $c|\operatorname{Re} z|, c>0$,

$$
\begin{aligned}
\log a(z) & =\log \left(\frac{z-i \mu_{1}}{z+i \mu_{1}}\right)-l(z) \\
& =\frac{1}{z}\left[-2 i \mu_{1}+\frac{1}{2 \pi i} \int_{\mathbb{R}} \log \left(1+|r(s)|^{2}\right) \mathrm{d} s\right]+O\left(\frac{1}{z^{2}}\right),
\end{aligned}
$$

where $l(z)$ is given in (3.25) and so we have

$$
\mu_{1}=\mu_{0}+\frac{1}{2} \int_{\mathbb{R}} \eta_{\mu_{0}}\left(w^{e}+\overline{w^{e}}\right)+\left|w^{e}\right|^{2}-\frac{1}{4 \pi} \int_{\mathbb{R}} \log \left(1+|r|^{2}\right) .
$$

This formula should be compared with Lemma 7.4. We see, in particular, that the contribution of $w^{e} \equiv u_{0}^{e}-\eta_{\mu_{0}}$ is approximated by $w_{1}$. Similarly, if $a(z)$ has two zeros,

$$
\begin{aligned}
& \mu_{1}+\mu_{2}=\frac{1}{2} \int_{\mathbb{R}}\left|u_{0}^{e}\right|^{2}-\frac{1}{4 \pi} \int_{\mathbb{R}} \log \left(1+|r(s)|^{2}\right) \mathrm{d} s \\
& \mu_{1}^{3}+\mu_{2}^{3}=\frac{3}{2} \int_{\mathbb{R}}\left(\left|u_{0}^{e}\right|^{4}-\left|\left(u_{0}^{e}\right)_{x}\right|^{2}\right)-\frac{3}{4 \pi} \int_{\mathbb{R}} s^{2} \log \left(1+|r(s)|^{2}\right) \mathrm{d} s .
\end{aligned}
$$

Proof of Theorem 1. Fix $0<\kappa<\frac{1}{4}$. It is enough to consider $x \geq 0$. First, we assume that $a(z)$ has one simple zero $z_{1}=i \mu_{1}$. From (4.39), we have $\beta=-q$. By (5.1) and Theorem 6, the norming constant $\gamma_{1}(t)=\gamma\left(t ; z_{1}\right)$ is given by

$$
\gamma_{1}(t)=\gamma_{1} e^{i z_{1}^{2} t / 2} \text { where } \gamma_{1}=e^{-i \rho_{1}} \sqrt{\frac{\mu_{1}+q}{\mu_{1}-q}} \text {, for some } \rho_{1} \in \mathbb{R} \text {. }
$$

Recalling the construction in the Appendix of the solution of a RHP with one pair of poles in terms of the solution of a RHP without poles using a Darboux transformation, we set

$$
r_{f}(z) \equiv r(z) \frac{z-\overline{z_{1}}}{z-z_{1}}, \quad z \in \mathbb{R}, \text { and } c_{1}(t)=\frac{\gamma_{1}(t)}{a^{\prime}\left(z_{1}\right)} .
$$

Note from (3.24) that $a(z)=\frac{z-i \mu_{1}}{z+i \mu_{1}} e^{-l(z)}$ where $l(z)$ is defined in (3.25). As $r(z)=\frac{\overline{b(z)}}{\overline{a(z)}}$, by (17.13) and (17.14), we see that

$$
\|r\|_{L^{\infty}} \leq c\|b\|_{L^{\infty}} \leq c \epsilon, \quad\|r\|_{L^{2}} \leq c\|b\|_{L^{2}} \leq c \epsilon,
$$

and

$$
\left\|r^{\prime}\right\|_{L^{2}}=\left\|\frac{b^{\prime}}{a}-\frac{b a^{\prime}}{a^{2}}\right\|_{L^{2}} \leq c\left(\left\|b^{\prime}\right\|_{L^{2}}+\|b\|_{L^{\infty}}\left\|a^{\prime}\right\|_{L^{2}}\right) \leq c \epsilon|q|^{-\frac{1}{2}} .
$$

Therefore,

$$
\left\|r_{f}\right\|_{L^{\infty}}=\|r\|_{L^{\infty}} \leq c \epsilon, \quad\left\|r_{f}\right\|_{H^{1,0}} \leq c\|r\|_{H^{1,0}} \leq c \epsilon|q|^{-\frac{1}{2}}
$$


Assume that $\epsilon \leq \chi_{0}|q|^{\frac{1}{2}}$ where $\chi_{0}>0$ will be determined further on. Denote by $\left(m_{f}\right)_{ \pm} \in I+\partial C\left(L^{2}\right)$ the solution of the normalized $\operatorname{RHP}\left(\mathbb{R}, v_{f}(z)\right)$ without poles where $v_{f}(z)=\left(\frac{1+\left|r_{f}(z)\right|^{2}}{r_{f}(z) e^{-i \theta}} e^{-(z) e^{i \theta}}\right)$. Such a solution exists by the general theory of Section 5. As before, $m_{f}(x, t, z)$, $z \in \mathbb{C} \backslash \mathbb{R}$, denote the extension of $\left(m_{f}\right)_{ \pm}$off the axis. We now apply the steepest-descent analysis in Section 5 with $r(z)$ replaced by $r_{f}(z)$. In the analysis in Section 5, various auxiliary functions such as $m_{d}^{L}$ etc. are introduced: in the present context we should properly use the notation $\left(m_{f}\right)_{d}^{L}$ etc., but we simply write $m_{d}^{L}$.

Let $\zeta_{1}=\sqrt{t}\left(z_{1}-z_{0}\right)=\sqrt{t}\left(i \mu_{1}-z_{0}\right)$. By the estimate (5.32), Lemma 5.21 and (6.2) for $t \geq 1$,

$$
\begin{aligned}
m_{f}\left(z_{1}\right)= & m_{d}^{L}\left(z_{1}\right) \Phi^{-1}\left(z_{1}\right) \delta\left(z_{1}\right)^{\sigma_{3}}+O\left(\epsilon|q|^{-\frac{1}{2}} t^{-\left(\frac{1}{2}+\kappa\right)}\right) \\
= & M^{\infty}\left(\zeta_{1}\right) \phi^{-\sigma}\left(\zeta_{1}\right) \Phi^{-1}\left(z_{1}\right) \delta\left(z_{1}\right)^{\sigma_{3}}+O\left(\epsilon|q|^{-\frac{1}{2}} t^{-\left(\frac{1}{2}+\kappa\right)}\right) \\
= & M_{\mathrm{II}}^{\infty}\left(\zeta_{1}\right) \phi^{-\sigma}\left(\zeta_{1}\right) \phi^{\mathrm{ad} \sigma}\left(K^{\infty}\right)\left(\zeta_{1}\right) \Phi^{-1}\left(z_{1}\right) \delta\left(z_{1}\right)^{\sigma_{3}} \\
& \quad+O\left(\epsilon|q|^{-\frac{1}{2}} t^{-\left(\frac{1}{2}+\kappa\right)}\right) .
\end{aligned}
$$

where $\Phi$ is defined in (5.15) with $r_{f}(z)$ and

$$
K^{\infty}(\zeta)= \begin{cases}I, & \zeta \in \mathrm{II}_{z_{0}}, \\
\left(\begin{array}{cc}
1 & \frac{r_{f}\left(z_{0}\right)}{1+\left|r_{f}\left(z_{0}\right)\right|^{2}} \\
0 & 1
\end{array}\right)^{-1}, \zeta \in \mathrm{III}_{z_{0}} .\end{cases}
$$

Note that if $\zeta_{1} \in \mathrm{II}_{z_{0}}$,

$$
\phi^{\operatorname{ad} \sigma}\left(K^{\infty}\right)\left(\zeta_{1}\right)=I, \quad \Phi^{-1}\left(z_{1}\right)=I,
$$

If $\zeta_{1} \in \operatorname{III}_{z_{0}}$, then $x \geq \mu_{1} t$ and hence $\left|e^{i \theta\left(\zeta_{1}\right)}\right|=\left|e^{-i \zeta_{1}^{2} / 2}\right|=e^{-\mu_{1} x}$ decay exponentially as $t \rightarrow \infty$. As $r_{f}\left(z_{0}\right)=O(\epsilon)$,

$$
\phi^{\operatorname{ad} \sigma}\left(K^{\infty}\right)\left(\zeta_{1}\right)=I+O\left(\epsilon e^{-c t}\right), \quad \Phi^{-1}\left(z_{1}\right)=I+O\left(\epsilon e^{-c t}\right) .
$$

Let $k_{1}, k_{2}$ be given in (6.12) and (6.14) with $r_{f}(z)$. We use from AS65] the identity $D_{a}^{\prime}(\eta)=-\frac{1}{2} \eta D_{a}(\eta)+a D_{a-1}(\eta)$ for $\eta \in \mathbb{C}$ to obtain

$$
\begin{aligned}
\partial_{\zeta}\left(D_{a}\left(e^{-\frac{3 i \pi}{4}} \zeta\right)\right) & =e^{-\frac{3 i \pi}{4}} D_{a}^{\prime}\left(e^{-\frac{3 i \pi}{4}} \zeta\right) \\
& =e^{-\frac{3 i \pi}{4}}\left[-\frac{1}{2} e^{-\frac{3 i \pi}{4}} \zeta D_{a}\left(e^{-\frac{3 i \pi}{4}} \zeta\right)+a D_{a-1}\left(e^{-\frac{3 i \pi}{4}} \zeta\right)\right] \\
& =-\frac{i}{2} \zeta D_{a}\left(e^{-\frac{3 i \pi}{4}} \zeta\right)+a e^{-\frac{3 i \pi}{4}} D_{a-1}\left(e^{-\frac{3 i \pi}{4}} \zeta\right)
\end{aligned}
$$

and hence by (6.9),

$$
\left(M_{\mathrm{II}}^{\infty}\right)_{21}(\zeta)=\frac{\alpha_{0}}{k_{1}} e^{\frac{3 \pi}{4} \nu\left(z_{0}\right)} a e^{-\frac{3 i \pi}{4}} D_{a-1}\left(e^{-\frac{3 i \pi}{4}} \zeta\right)
$$


Similarly, it follows from (6.10) that

$$
\left(M_{\mathrm{II}}^{\infty}\right)_{12}(\zeta)=-\frac{\alpha_{0}^{-1}}{k_{2}} e^{-\frac{\pi}{4} \nu\left(z_{0}\right)} a e^{-\frac{i \pi}{4}} D_{-a-1}\left(e^{-\frac{i \pi}{4}} \zeta\right)
$$

Thus as $a=-i \nu\left(z_{0}\right)=O\left(\epsilon^{2}\right)$, we see from asymptotic expansion (6.8) of $D_{a}(\cdot)$ that

$$
M_{\mathrm{II}}^{\infty}\left(\zeta_{1}\right) \phi^{-\sigma}\left(\zeta_{1}\right)=\left(\begin{array}{cc}
1 & p_{1} \\
p_{2} & 1
\end{array}\right)+O\left(\epsilon^{2} t^{-1}\right)
$$

where

$$
p_{1}=-\frac{i k_{1}}{\zeta_{1}}=\frac{i k_{1}}{\sqrt{t}\left(z_{0}-i \mu_{1}\right)} \text { and } p_{2}=\frac{i k_{2}}{\zeta_{1}}=-\frac{i k_{2}}{\sqrt{t}\left(z_{0}-i \mu_{1}\right)} .
$$

Let $\delta(z)$ be given in (5.13) with $r_{f}(z)$. Since $\left|r_{f}(z)\right|=|r(z)|=|r(-z)|=$ $\left|r_{f}(-z)\right|$ for $z \in \mathbb{R}$ by Remark 4.30, we have $e^{l\left(z_{1}\right)} \delta\left(z_{1}\right)^{-2}=e^{-i l_{1}} \hat{\delta}_{1}^{-2}$ where $l_{1}, \delta_{1}$ are given in (1.11) and (1.12), respectively. Assembling the above results, we obtain

$$
\begin{aligned}
\mathfrak{b}(x, t) & \equiv m_{f}\left(z_{1}\right) e^{i x z_{1} \sigma}\left(\begin{array}{c}
1 \\
\frac{-c_{1}(t)}{z_{1}-z_{1}}
\end{array}\right) \\
& =\left(M_{\mathrm{II}}^{\infty}\left(\zeta_{1}\right) \phi^{-\sigma}\left(\zeta_{1}\right)+O\left(\epsilon|q|^{-\frac{1}{2}} t^{-\left(\frac{1}{2}+\kappa\right)}\right)\right) \delta\left(z_{1}\right)^{\sigma 3} e^{i x z_{1} \sigma}\left(\begin{array}{c}
1 \\
\frac{-c_{1}(t)}{z_{1}-\overline{z_{1}}}
\end{array}\right) \\
& =v_{1}^{-1} \delta\left(z_{1}\right) e^{\frac{\mu_{1} x}{2}}\left[\left(\begin{array}{c}
v_{1}^{x}+p_{1} \\
p_{2} v_{1}^{x}+1
\end{array}\right)+O\left(\epsilon|q|^{-\frac{1}{2}} t^{-\left(\frac{1}{2}+\kappa\right)}\right)\right]
\end{aligned}
$$

where $v_{1}, v_{1}^{x}$ are defined in (1.13). For $u_{f}(x, t)$, we have the expansion $m_{d}^{L}(z)=M_{d}^{L}(\zeta)=I+\left(M_{d}^{L}\right)_{1} \zeta^{-1}+o\left(\zeta^{-1}\right)=I+\left(M_{d}^{L}\right)_{1}(\sqrt{t} z)^{-1}+o\left(z^{-1}\right)$ as $z \rightarrow \infty$, which implies that $\left(m_{d}^{L}\right)_{1}=\frac{1}{\sqrt{t}}\left(M_{d}^{L}\right)_{1}$. Hence, by (5.31) and (6.4),

$$
\begin{aligned}
u_{f}(x, t) & =-i\left(\left(m_{d}^{L}\right)_{1}\right)_{12}+O\left(\epsilon|q|^{-\frac{1}{2}} t^{-\left(\frac{1}{2}+\kappa\right)}\right) \\
& =-\frac{i}{\sqrt{t}}\left(\left(M_{d}^{L}\right)_{1}\right)_{12}+O\left(\epsilon|q|^{-\frac{1}{2}} t^{-\left(\frac{1}{2}+\kappa\right)}\right) \\
& =-\frac{k_{1}}{\sqrt{t}}+O\left(\epsilon|q|^{-\frac{1}{2}} t^{-\left(\frac{1}{2}+\kappa\right)}\right) .
\end{aligned}
$$

It follows by the Darboux transformation that

$$
\begin{aligned}
u(x, t) & =u_{f}(x, t)+i\left(z_{1}-\overline{z_{1}}\right) \mathcal{F}(\mathfrak{b}(x, t)) \\
& =-\frac{k_{1}}{\sqrt{t}}-\frac{2 \mu_{1}\left(v_{1}^{x}+p_{1}\right)\left(\overline{p_{2}} \overline{v_{1}^{x}}+1\right)}{\left|v_{1}^{x}+p_{1}\right|^{2}+\left|p_{2} v_{1}^{x}+1\right|^{2}}+O\left(\epsilon|q|^{-\frac{1}{2}} t^{-\left(\frac{1}{2}+\kappa\right)}\right),
\end{aligned}
$$

where $\mathcal{F}$ is defined in (4.3). This equation holds, in particular, for $x \geq 1 / M$. 
For $0 \leq x \leq M, z_{0}=O\left(t^{-1}\right)$ and hence $\hat{\delta}_{1}=1+O\left(\epsilon^{2} t^{-1}\right)$. By Remark 4.30, $r_{f}(0)=r(0)=0$. Since $r_{f}(z)=\int_{0}^{z} r_{f}^{\prime}(s) \mathrm{d} s$, we have $r_{f}\left(z_{0}\right)=O\left(\epsilon|q|^{-\frac{1}{2}}\left|z_{0}\right|^{\frac{1}{2}}\right)=O\left(\epsilon|q|^{-\frac{1}{2}} t^{-\frac{1}{2}}\right)$ and hence $k_{1}, k_{2}=O\left(\epsilon|q|^{-\frac{1}{2}} t^{-\frac{1}{2}}\right)$. Thus,

$$
u_{f}(x, t)=O\left(\epsilon|q|^{-\frac{1}{2}} t^{-\left(\frac{1}{2}+\kappa\right)}\right), \quad p_{1}, p_{2}=O\left(\epsilon|q|^{-\frac{1}{2}} t^{-1}\right) .
$$

and hence it follows by (7.31) that

$$
\begin{aligned}
u(x, t) & =\frac{-2 \mu_{1} v_{1}^{x}}{\left|v_{1}^{x}\right|^{2}+1}+O\left(\epsilon|q|^{-\frac{1}{2}} t^{-\left(\frac{1}{2}+\kappa\right)}\right) \\
& =e^{i\left(\mu_{1}^{2} t / 2+\rho_{1}+l_{1}\right)} v_{\mu_{1}}(x)+O\left(\epsilon|q|^{-\frac{1}{2}} t^{-\left(\frac{1}{2}+\kappa\right)}\right),
\end{aligned}
$$

Now, suppose that $a(z)$ has two zeros $z_{1}=i \mu_{1}$ and $z_{2}=i \mu_{2}$. We have $\beta=q$ from (4.39). Again by (5.1) and Theorem 6, the norming constants $\gamma_{j}(t)=\gamma\left(t ; z_{j}\right), j=1,2$ are given by

$$
\gamma_{j}(t)=\gamma_{j} e^{i z_{j}^{2} t / 2}, \text { where } \gamma_{j}=e^{-i \rho_{j}} \sqrt{\frac{\mu_{j}-q}{\mu_{j}+q}}, \text { for some } \rho_{j} \in \mathbb{R} \text {. }
$$

Set $\zeta_{2}=\sqrt{t}\left(z_{2}-z_{0}\right)=\sqrt{t}\left(i \mu_{2}-z_{0}\right)$. Recalling from the Appendix the action of repeated Darboux transformation, we set

$$
r_{f}(z) \equiv r(z) \frac{z-\overline{z_{1}}}{z-z_{1}} \frac{z-\overline{z_{2}}}{z-z_{2}}, \quad \text { and } c_{1}(t)=\frac{\gamma_{1}(t)}{a_{1}^{\prime}\left(z_{1}\right)}, c_{2}(t)=\frac{\gamma_{2}(t)}{a^{\prime}\left(z_{2}\right)}
$$

where $a_{1}(z)=\frac{z-z_{1}}{z-z_{1}} e^{-l(z)}$ and $a(z)=\frac{z-z_{2}}{z-z_{2}} a_{1}(z)$. Denote by $m_{1}(x, t, z)$ the solution of the normalized RHP with the reflection coefficient $r_{1}(z) \equiv$ $r(z) \frac{z-\overline{z_{2}}}{z-z_{2}}$ and one pair of simple poles at $z= \pm i \mu_{1}$ whose norming constants are $\gamma_{1}(t),-\overline{\gamma_{1}(t)}$, respectively. We have

$$
\begin{aligned}
\mathfrak{b}_{1}(x, t) & \equiv m_{f}\left(z_{1}\right) e^{i z_{1} x \sigma}\left(\begin{array}{c}
1 \\
\frac{-c_{1}(t)}{z_{1}-\overline{z_{1}}}
\end{array}\right) \\
& =\hat{v}_{1}^{-1} \delta\left(z_{1}\right) e^{\frac{\mu_{1} x}{2}}\left[\left(\begin{array}{c}
\hat{v}_{1}^{x}+p_{1} \\
p_{2} \hat{v}_{1}^{x}+1
\end{array}\right)+O\left(\epsilon|q|^{-\frac{1}{2}} t^{-\left(\frac{1}{2}+\kappa\right)}\right)\right] .
\end{aligned}
$$

where $\hat{v}_{1}, \hat{v}_{1}^{x}$ are given in (1.13). Write $\mathfrak{b}_{1}=\left(\left(\mathfrak{b}_{1}\right)_{1},\left(\mathfrak{b}_{1}\right)_{2}\right)^{T}$ and define

$$
\begin{aligned}
& \lambda_{1} \equiv \frac{z_{2}-\overline{z_{1}}}{z_{2}-z_{1}}, \quad \hat{\mathfrak{b}}_{1} \equiv\left(\begin{array}{cc}
\left(\mathfrak{b}_{1}\right)_{1} & -\left(\overline{\mathfrak{b}_{1}}\right)_{2} \\
\left(\mathfrak{b}_{1}\right)_{2} & \left(\overline{\mathfrak{b}_{1}}\right)_{1}
\end{array}\right) \\
& \hat{\mu} \equiv\left(\begin{array}{cc}
z_{2}-z_{1} & 0 \\
0 & z_{2}-\overline{z_{1}}
\end{array}\right)=\left(z_{2}-z_{1}\right)\left(\begin{array}{cc}
1 & 0 \\
0 & \lambda_{1}
\end{array}\right),
\end{aligned}
$$


Let $\psi_{1}(x, t, z)=m_{1}(x, t, z) e^{i x z \sigma}$. It follows from the Darboux transformation (see (9.2)) that

$$
\begin{aligned}
\mathfrak{b}_{2} & \equiv \psi_{1}\left(z_{2}\right)\left(\begin{array}{c}
1 \\
\frac{-c_{2}(t)}{z_{2}-\overline{z_{2}}}
\end{array}\right) \\
& =\hat{\mathfrak{b}}_{1} \hat{\mu} \hat{\mathfrak{b}}_{1}^{-1}\left(m_{f}\left(z_{2}\right) e^{i x z_{2} \sigma}\right) \hat{\mu}^{-1}\left(\begin{array}{c}
1 \\
\frac{-c_{2}(t)}{z_{2}-\overline{z_{2}}}
\end{array}\right) \\
& =\hat{\mathfrak{b}}_{1} \hat{\mu} \hat{\mathfrak{b}}_{1}^{-1}\left(m_{f}\left(z_{2}\right) \delta\left(z_{2}\right)^{-\sigma_{3}}\right)\left(\begin{array}{c}
\hat{v}_{2}^{x} \\
1
\end{array}\right) \frac{e^{\frac{\mu_{2} x}{2}} \delta\left(z_{2}\right)}{\hat{v}_{2}\left(z_{2}-z_{1}\right)} .
\end{aligned}
$$

where $\hat{v}_{2}, \hat{v}_{2}^{x}$ are given in (1.13). As $z_{2}=-i q+O\left(\epsilon^{2} q\right)$ by Lemma 7.4, we have

$$
\left\|\frac{r_{f}(\cdot)}{\cdot-z_{2}}\right\|_{H^{1,0}} \leq c \epsilon|q|^{-\frac{3}{2}} .
$$

Now set $\tau=|q| \sqrt{t}$ and assume $t \gg q^{-2}$. Then, $\tau \gg 1$. It follows by (7.29) that

$$
\phi^{\operatorname{ad} \sigma}\left(K^{\infty}\right)\left(\zeta_{2}\right) \Phi^{-1}\left(z_{2}\right)= \begin{cases}I+O\left(\epsilon e^{-\mu_{2} x}\right)=I+O\left(\epsilon e^{-\tau^{2}}\right), & \text { if } x \geq \mu_{2} t \\ I & , \text { otherwise }\end{cases}
$$

As $\left|\zeta_{2}\right|^{-2}=\left(t\left(z_{0}^{2}+\mu_{2}^{2}\right)\right)^{-1}=O\left(\tau^{-2}\right)$, it follows by (6.8) again that

$$
M_{\mathrm{II}}^{\infty}\left(\zeta_{2}\right) \phi^{-\sigma}\left(\zeta_{2}\right)=\left(\begin{array}{cc}
1 & p_{3} \\
p_{4} & 1
\end{array}\right)+O\left(\epsilon^{2}\left(z_{0}^{2}+q^{2}\right)^{-1} t^{-1}\right),
$$

where

$$
p_{3}=-\frac{i k_{1}}{\zeta_{2}}=\frac{i k_{1}}{\sqrt{t}\left(z_{0}-i \mu_{2}\right)}, \quad p_{4}=\frac{i k_{2}}{\zeta_{2}}=-\frac{i k_{2}}{\sqrt{t}\left(z_{0}-i \mu_{2}\right)},
$$

Thus, using Lemma 5.21 again, we see that

$$
\begin{aligned}
m_{f}\left(z_{2}\right) & \delta\left(z_{2}\right)^{-\sigma_{3}} \\
= & M_{\mathrm{II}}^{\infty}\left(\zeta_{2}\right) \phi^{-\sigma}\left(\zeta_{2}\right) \phi^{\mathrm{ad} \sigma}\left(K^{\infty}\right)\left(\zeta_{2}\right) \Phi^{-1}\left(z_{2}\right)+O\left(\epsilon|q|^{-\frac{3}{2}} t^{-\left(\frac{1}{2}+\kappa\right)}\right) \\
= & \left(\begin{array}{cc}
1 & p_{3} \\
p_{4} & 1
\end{array}\right)+O\left(\epsilon e^{-\tau^{2}}+\epsilon^{2}\left(z_{0}^{2}+q^{2}\right)^{-1} t^{-1}+\epsilon|q|^{-\frac{3}{2}} t^{-\left(\frac{1}{2}+\kappa\right)}\right)
\end{aligned}
$$

where $\epsilon e^{-\tau^{2}}$ is dropped from the error term if $x<\mu_{2} t$. Inserting $\hat{\mathfrak{b}}_{1} \hat{\mu} \hat{\mathfrak{b}}_{1}^{-1}=\frac{z_{2}-z_{1}}{\left|\left(\mathfrak{b}_{1}\right)_{1}\right|^{2}+\left|\left(\mathfrak{b}_{1}\right)_{2}\right|^{2}}\left(\begin{array}{ll}\mid \frac{\left.\left(\mathfrak{b}_{1}\right)_{1}\right|^{2}+\lambda_{1}\left|\left(\mathfrak{b}_{1}\right)_{2}\right|^{2}}{\left(\mathfrak{b}_{1}\right)_{1}}\left(\mathfrak{b}_{1}\right)_{1} \overline{\left(\mathfrak{b}_{1}\right)_{2}}\left(1-\lambda_{1}\right) \\ \left(1-\lambda_{1}\right) & \lambda_{1}\left|\left(\mathfrak{b}_{1}\right)_{1}\right|^{2}+\left|\left(\mathfrak{b}_{1}\right)_{2}\right|^{2}\end{array}\right)$ into (․33) and assembling the above results, we obtain

$$
\begin{aligned}
\mathfrak{b}_{2}=e^{\frac{\mu_{2} x}{2}} \delta\left(z_{2}\right) \hat{v}_{2}\left[\left(\begin{array}{l}
s_{1} \\
s_{2}
\end{array}\right)\right. & \\
& \left.+O\left(\epsilon e^{-\tau^{2}}+\epsilon^{2}\left(z_{0}^{2}+q^{2}\right)^{-1} t^{-1}+\epsilon|q|^{-\frac{3}{2}} t^{-\left(\frac{1}{2}+\kappa\right)}\right)\right],
\end{aligned}
$$


where $s_{1}$ and $s_{2}$ are given, after some calculations, by (1.20). Again by the Darboux transformation, it follows that

$$
\begin{aligned}
& u(x, t) \\
& =u_{f}(x, t)+i\left(z_{1}-\overline{z_{1}}\right) \mathcal{F}\left(\mathfrak{b}_{1}(x, t)\right)+i\left(z_{2}-\overline{z_{2}}\right) \mathcal{F}\left(\mathfrak{b}_{2}(x, t)\right), \\
& =-\frac{k_{1}}{\sqrt{t}}-\frac{2 \mu_{1}\left(\hat{v}_{1}^{x}+p_{1}\right)\left(\overline{p_{2}} \overline{\hat{v}_{1}^{x}}+1\right)}{\left|\hat{v}_{1}^{x}+p_{1}\right|^{2}+\left|p_{2} \hat{v}_{1}^{x}+1\right|^{2}}-\frac{2 \mu_{2} s_{1} \overline{s_{2}}}{\left|s_{1}\right|^{2}+\left|s_{2}\right|^{2}} \\
& \quad+O\left(\epsilon q e^{-\tau^{2}}+\epsilon^{2} q\left(z_{0}^{2}+q^{2}\right)^{-1} t^{-1}+\epsilon|q|^{-\frac{1}{2}} t^{-\left(\frac{1}{2}+\kappa\right)}\right) .
\end{aligned}
$$

This equation holds, in particular, for $x \geq 1 / M$.

If $0 \leq x \leq M$, we have $z_{0}=O\left(t^{-1}\right)$ and $k_{1}, k_{2}=O\left(\epsilon|q|^{-\frac{1}{2}} t^{-\frac{1}{2}}\right)$ as above. Hence $p_{1}, p_{2}=O\left(\epsilon|q|^{-\frac{1}{2}} t^{-1}\right)$ and $p_{3}, p_{4}=O\left(\epsilon|q|^{-\frac{3}{2}} t^{-1}\right)$. Thus,

$$
\begin{aligned}
u(x, t)= & -\frac{2 \mu_{1} \hat{v}_{1}^{x}}{\left|\hat{v}_{1}^{x}\right|^{2}+1}-\frac{2 \mu_{2} s_{1} \overline{s_{2}}}{\left|s_{1}\right|^{2}+\left|s_{2}\right|^{2}}+O\left(\epsilon|q|^{-\frac{1}{2}} t^{-\left(\frac{1}{2}+\kappa\right)}\right), \\
= & e^{i\left(\mu_{1}^{2} t / 2+\rho_{1}+l_{1}\right)} \mu_{1} \operatorname{sech}\left(\mu_{1} x-\tanh ^{-1}\left(q / \mu_{1}\right)\right) \\
& -\frac{2 \mu_{2}\left(\hat{v}_{2}^{x}-s_{0}\right)\left(1+\hat{v}_{1}^{x} \overline{s_{0}}\right)}{\left|\hat{v}_{2}^{x}-s_{0}\right|^{2}+\left|1+\hat{v}_{1}^{x} \overline{s_{0}}\right|^{2}}+O\left(\epsilon|q|^{-\frac{1}{2}} t^{-\left(\frac{1}{2}+\kappa\right)}\right),
\end{aligned}
$$

where $s_{0}$ is given in (1.17). This completes the proof of Theorem 1 .

\section{Proof of Theorem 2}

Lemma 8.1. Let $u(x)$ be a real-valued function on the half line $x \geq 0$ and let $u^{e}(x)$ be the Bäcklund extension of $u(x)$ with respect to $q$. Let $\psi^{+}(x, z)$ be the $Z S-A K N S$ solution associated with $u^{e}$. Then,

$$
\psi^{+}(x, z)=\overline{\psi^{+}(x,-z)}, \quad x \geq 0, \quad z \in \mathbb{R} .
$$

Suppose that the scattering function $a(z)$ of $u^{e}(x)$ has $n$ simple zeros in $\mathbb{C}^{+}$. If $z=i \mu \in i \mathbb{R}^{+}$is a zero of $a(z)$, the corresponding norming constant $\gamma(i \mu)$ of $z=i \mu$ is real. For $u(x)=v_{\mu_{0}}(x)+q w(x)$, w real, $q \ll 1$, in particular, we have

$$
\gamma(i \mu)=\sqrt{\frac{\mu-\beta}{\mu+\beta}}, \quad \beta=(-1)^{n} q .
$$

Proof. Let $Q(x)=\left(\begin{array}{cc}\frac{0}{-u_{(x)}} & 0(x)\end{array}\right)$. As $u(x), x \geq 0$, is real, we see that

$$
\overline{\psi_{x}^{+}(x,-z)}=(i z \sigma+Q) \overline{\psi^{+}(x,-z)}, \quad x \geq 0, \quad z \in \mathbb{R} .
$$

and $\overline{\psi^{+}(x,-z)} e^{-i x z \sigma}=\overline{\psi^{+}(x,-z) e^{-i x(-z) \sigma}} \rightarrow I$ as $x \rightarrow \infty$, which implies (8.1). 
For the norming constant $\gamma(i \mu)$, as $\mu$ and $u(x), x \geq 0$, are real, we have

$$
\overline{\psi_{x}^{+}(x, i \mu)}=(i(i \mu) \sigma+Q) \overline{\psi^{+}(x, i \mu)}, \quad x \geq 0,
$$

and $\overline{\psi^{+}(x, i \mu)} e^{-i x(i \mu) \sigma} \rightarrow \mathrm{I}$ as $x \rightarrow+\infty$, which implies that $\psi^{+}(x, i \mu)=$ $\overline{\psi^{+}(x, i \mu)}, x \geq 0$. Therefore, $\psi^{+}(0, i \mu)$ is real and hence the result follows from (4.39) and (4.54).

Define

$$
E_{+}^{0}(z) \equiv \int_{z}^{\infty} e^{-i s^{2} / 2} \mathrm{~d} s, \quad E_{+}(z) \equiv \int_{z}^{\infty} e^{-i t s^{2} / 2} \mathrm{~d} s=\frac{1}{\sqrt{t}} E_{+}^{0}(\sqrt{t} z),
$$

and

$$
E_{-}^{0}(z) \equiv \int_{-\infty}^{z} e^{-i s^{2} / 2} \mathrm{~d} s, \quad E_{-}(z) \equiv \int_{-\infty}^{z} e^{-i t s^{2} / 2} \mathrm{~d} s=\frac{1}{\sqrt{t}} E_{-}^{0}(\sqrt{t} z),
$$

Note that $E_{0} \equiv \int_{-\infty}^{\infty} e^{-i s^{2} / 2} \mathrm{~d} s=\sqrt{2 \pi} e^{-\frac{i \pi}{4}}$.

Lemma 8.2. For any $g \in L^{2}\left(\mathbb{R}^{+}\right), t \geq 1$,

$$
\int_{0}^{\infty} g(z) E_{+}(z) \mathrm{d} z=O\left(\frac{\|g\|_{L^{2}}}{t^{3 / 4}}\right) .
$$

Proof. As $g \in L^{2}\left(\mathbb{R}^{+}\right)$, it follows that

$$
\begin{gathered}
\left|\int_{0}^{\infty} g(z) E_{+}(z) \mathrm{d} z\right|=\left|\int_{0}^{1 / \sqrt{t}}+\int_{1 / \sqrt{t}}^{\infty} g(z) \frac{E_{+}^{0}(\sqrt{t} z)}{\sqrt{t}} \mathrm{~d} z\right| \\
\leq \frac{c}{\sqrt{t}}\left[\|g\|_{L^{2}} \cdot \frac{1}{t^{1 / 4}}+\|g\|_{L^{2}} \cdot\left(\int_{1 / \sqrt{t}}^{\infty}\left|E_{+}^{0}(\sqrt{t} z)\right|^{2} \mathrm{~d} z\right)^{\frac{1}{2}}\right] .
\end{gathered}
$$

Since $E_{+}^{0}(z)=\frac{1}{i z} e^{-i z^{2} / 2}+O\left(\frac{1}{z^{2}}\right)$ as $z \rightarrow \infty$, we have

$$
\int_{1 / \sqrt{t}}^{\infty}\left|E_{+}^{0}(\sqrt{t} z)\right|^{2} \mathrm{~d} z=\frac{1}{\sqrt{t}} \int_{1}^{\infty}\left|E_{+}^{0}(u)\right|^{2} \mathrm{~d} u=O\left(\frac{1}{\sqrt{t}}\right) .
$$

Lemma 8.3. Let $|q| \ll 1, \tau=|q| \sqrt{t}$ and $z_{0} \in \mathbb{R}$. Suppose that

$$
\begin{aligned}
& g(z)=g_{1}(z)+h(z) g_{2}(z), \\
& h(z)=\prod_{k=1}^{n} \frac{i q_{k}}{z+i q_{k}}, \quad q_{k}= \pm q, \quad 1 \leq k \leq n, \text { and } \\
& \left\|g_{i}\right\|_{H^{1,0}(\mathbb{R})} \leq c|q|, \quad i=1,2 .
\end{aligned}
$$


Then, for $C>1$,

$$
\begin{aligned}
& \int_{-\infty}^{\infty} g(z) e^{-i t\left(z-z_{0}\right)^{2} / 2} \mathrm{~d} z \\
& \quad=\left\{\begin{array}{l}
\frac{g_{1}\left(z_{0}\right)}{\sqrt{t}} E_{0}+O\left(\frac{q}{\sqrt{t}}\left(|q|^{\frac{1}{2}}+t^{-\frac{1}{4}}+\tau|\log \tau|\right)\right), \quad \tau \leq \frac{1}{2} \\
O\left(q^{2}\right), \quad \tau \geq 1 / C>0 .
\end{array}\right.
\end{aligned}
$$

Proof. We first consider $z>z_{0}$. By integrating by parts and using Lemma 8.2, we see that

$$
\begin{aligned}
\int_{z_{0}}^{\infty} & g_{1}(z) e^{-i t\left(z-z_{0}\right)^{2} / 2} \mathrm{~d} z \\
& =-\left.g_{1}(z) E_{+}\left(z-z_{0}\right)\right|_{z_{0}} ^{\infty}+\int_{z_{0}}^{\infty} g_{1}^{\prime}(z) E_{+}\left(z-z_{0}\right) \mathrm{d} z \\
& =\frac{g_{1}\left(z_{0}\right)}{\sqrt{t}} E_{+}^{0}(0)+O\left(\frac{q}{t^{3 / 4}}\right) .
\end{aligned}
$$

Similarly, we have

$$
\begin{aligned}
\int_{z_{0}}^{\infty} & h(z) g_{2}(z) e^{-i t\left(z-z_{0}\right)^{2} / 2} \mathrm{~d} z \\
= & -\left.h(z) g_{2}(z) E_{+}\left(z-z_{0}\right)\right|_{z_{0}} ^{\infty}+\int_{z_{0}}^{\infty} h(z) g_{2}^{\prime}(z) E_{+}\left(z-z_{0}\right) \mathrm{d} z \\
& \quad+\int_{z_{0}}^{\infty} h^{\prime}(z) g_{2}(z) E_{+}\left(z-z_{0}\right) \mathrm{d} z \\
= & \frac{g_{2}\left(z_{0}\right)}{\sqrt{t}} h\left(z_{0}\right) E_{+}^{0}(0)+\mathrm{I}+\mathrm{II} .
\end{aligned}
$$

Since $\left\|h \cdot g_{2}^{\prime}\right\|_{L^{2}} \leq c|q|, \mathrm{I}=O\left(\frac{q}{t^{3 / 4}}\right)$ by Lemma 8.2. Let $\hat{z_{0}}=z_{0} /|q|$ and $h_{1}(u)=\prod_{k=1}^{n} \frac{i q_{k} /|q|}{u+i q_{k} /|q|}$. By change of variable $z=|q| u$,

$$
\begin{aligned}
\mathrm{II}= & \frac{1}{\sqrt{t}} \int_{\hat{z_{0}}}^{\infty} h_{1}^{\prime}(u)\left(g_{2}(|q| u)-g_{2}(0)\right) E_{+}^{0}\left(\tau u-\sqrt{t} z_{0}\right) \mathrm{d} u \\
& +\frac{g_{2}(0)}{\sqrt{t}} \int_{\hat{z_{0}}}^{\infty} h_{1}^{\prime}(u)\left(E_{+}^{0}\left(\tau u-\sqrt{t} z_{0}\right)-E_{+}^{0}\left(-\sqrt{t} z_{0}\right)\right) \mathrm{d} u \\
& +\frac{g_{2}(0)}{\sqrt{t}}\left(-h_{1}\left(\hat{z_{0}}\right)\right) E_{+}^{0}\left(-\sqrt{t} z_{0}\right) \\
= & \mathrm{II}_{1}+\mathrm{II}_{2}-\frac{g_{2}(0)}{\sqrt{t}} h\left(z_{0}\right) E_{+}^{0}\left(-\sqrt{t} z_{0}\right) .
\end{aligned}
$$


As $\left|g_{2}(|q| u)-g_{2}(0)\right|=\left|\int_{0}^{|q| u} g_{2}^{\prime}\right| \leq|q u|^{\frac{1}{2}}\left\|g_{2}^{\prime}\right\|_{L^{2}}$,

$$
\left|\mathrm{II}_{1}\right| \leq \frac{|q|^{\frac{1}{2}}\left\|g_{2}^{\prime}\right\|_{L^{2}}}{\sqrt{t}} \int_{\hat{z_{0}}}^{\infty}\left|h_{1}^{\prime}(u)\right||u|^{\frac{1}{2}} \mathrm{~d} u=O\left(\frac{|q|^{3 / 2}}{\sqrt{t}}\right) .
$$

If $\tau \leq \frac{1}{2},\left|E_{+}^{0}\left(\tau u-\sqrt{t} z_{0}\right)-E_{+}^{0}\left(-\sqrt{t} z_{0}\right)\right| \leq \min \{c, \tau|u|\}$, and hence

$$
\begin{aligned}
\left|\mathrm{II}_{2}\right| & \leq \frac{c\left|g_{2}(0)\right|}{\sqrt{t}}\left[\int_{-\infty}^{-1 / \tau}\left|h_{1}^{\prime}(u)\right|+\int_{-1 / \tau}^{1 / \tau}\left|h_{1}^{\prime}(u)\right| \tau|u|+\int_{1 / \tau}^{\infty}\left|h_{1}^{\prime}(u)\right|\right] \\
& \leq \frac{c\left|g_{2}(0)\right|}{\sqrt{t}}\left[\tau \int_{0}^{1 / \tau} \frac{u}{u^{2}+1}+\int_{1 / \tau}^{\infty} \frac{1}{u^{2}}\right]=O\left(\frac{q}{\sqrt{t}} \tau|\log \tau|\right) .
\end{aligned}
$$

Assembling the above inequalities, we obtain

$$
\begin{aligned}
& \int_{z_{0}}^{\infty} g(z) e^{-i t\left(z-z_{0}\right)^{2} / 2} \mathrm{~d} z \\
& =\frac{g_{1}\left(z_{0}\right)}{\sqrt{t}} E_{+}^{0}(0)+\frac{h\left(z_{0}\right)}{\sqrt{t}}\left(g_{2}\left(z_{0}\right) E_{+}^{0}(0)-g_{2}(0) E_{+}^{0}\left(-\sqrt{t} z_{0}\right)\right) \\
& \quad+O\left(\frac{q}{\sqrt{t}}\left(|q|^{\frac{1}{2}}+t^{-\frac{1}{4}}+\tau|\log \tau|\right)\right)
\end{aligned}
$$

Similarly using $E_{-}^{0}(z), E_{-}(z)$, we obtain for $z<z_{0}$,

$$
\begin{aligned}
& \int_{-\infty}^{z_{0}} g(z) e^{-i t\left(z-z_{0}\right)^{2} / 2} \mathrm{~d} z \\
& =\frac{g_{1}\left(z_{0}\right)}{\sqrt{t}} E_{-}^{0}(0)+\frac{h\left(z_{0}\right)}{\sqrt{t}}\left(g_{2}\left(z_{0}\right) E_{-}^{0}(0)-g_{2}(0) E_{-}^{0}\left(-\sqrt{t} z_{0}\right)\right) \\
& \quad+O\left(\frac{q}{\sqrt{t}}\left(|q|^{\frac{1}{2}}+t^{-\frac{1}{4}}+\tau|\log \tau|\right)\right)
\end{aligned}
$$

and hence

$$
\begin{aligned}
& \int_{-\infty}^{\infty} g(z) e^{-i t\left(z-z_{0}\right)^{2} / 2} \mathrm{~d} z \\
& =\frac{E_{0}}{\sqrt{t}}\left(g_{1}\left(z_{0}\right)+h\left(z_{0}\right)\left(g_{2}\left(z_{0}\right)-g_{2}(0)\right)\right)+O\left(\frac{q}{\sqrt{t}}\left(|q|^{\frac{1}{2}}+t^{-\frac{1}{4}}+\tau|\log \tau|\right)\right)
\end{aligned}
$$

As

$$
\left|h\left(z_{0}\right)\left(g_{2}\left(z_{0}\right)-g_{2}(0)\right)\right| \leq \frac{c|q|}{\left|z_{0}\right|+|q|}\left\|g_{2}^{\prime}\right\|_{L^{2}}\left|z_{0}\right|^{\frac{1}{2}} \leq c|q|^{3 / 2}
$$

the result follows for $\tau \leq \frac{1}{2}$.

For $\tau \geq 1 / C>0$, note that $\frac{1}{\sqrt{t}} \leq C|q|$. The result follows from the fact that

$$
\left|\mathrm{II}_{2}\right| \leq \frac{c\left|g_{2}(0)\right|}{\sqrt{t}} \int_{\hat{z_{0}}}^{\infty}\left|h_{1}^{\prime}(u)\right| \mathrm{d} u=O\left(q^{2}\right)
$$


with the similar estimate for $z<z_{0}$.

Lemma 8.4. Let $m(x, t, z)$ solve the normalized $R H P(\mathbb{R}, v)$ without poles where the jump matrix $v$ is given in (5.8). Let $u(x, t)$ be the associated potential function. Suppose that $\|r\|_{L^{2} \cap L^{\infty}} \leq c|q| \ll 1$. Then, for each fixed $z \in \mathbb{C}^{+}$,

$$
m(x, t, z)=I+\frac{1}{2 \pi i} \int_{\mathbb{R}}\left(\begin{array}{cc}
0 & \frac{r(s)}{s-z} e^{i \theta(s)} \\
\frac{r(s)}{s-z} e^{-i \theta(s)} & 0
\end{array}\right) \mathrm{d} s+O\left(\frac{q^{2}}{|\operatorname{Im} z|^{\frac{1}{2}}}\right),
$$

and

$$
u(x, t)=\frac{1}{2 \pi} \int_{\mathbb{R}} r(s) e^{i \theta(s)} \mathrm{d} s+O\left(q^{2}\right) .
$$

where $\theta(z)=x z-\frac{1}{2} t z^{2}$.

Proof. We refer to Section 3 for the solution procedure for the RHP $(\mathbb{R}, v)$. Let $w_{+}=v-I$. Since $\|r\|_{L^{2} \cap L^{\infty}(\mathbb{R})} \leq c|q| \ll 1$,

$$
\left\|\left(1-C_{v}\right)^{-1}\right\|_{L^{2}(\mathbb{R})} \leq \frac{1}{1-\|r\|_{\infty}} \leq c,
$$

where $C_{v}$ is defined in (3.33). Hence

$$
\mu \equiv I+\left(1-C_{v}\right)^{-1} C_{v} I=I+\left(1-C_{v}\right)^{-1} C^{-} w_{+} \in I+L^{2}(\mathbb{R}) .
$$

Here

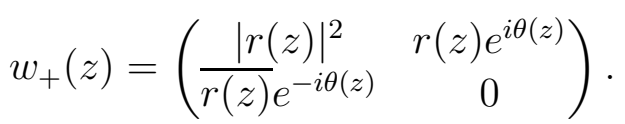

As

$$
\left|\int_{\mathbb{R}} \frac{g_{1}(s) g_{2}(s)}{s-z} \mathrm{~d} s\right| \leq \frac{\left\|g_{1}\right\|_{L^{2}}\left\|g_{2}\right\|_{L^{\infty}}}{|\operatorname{Im} z|^{\frac{1}{2}}}
$$

for any $g_{1} \in L^{2}(\mathbb{R}), g_{2} \in L^{\infty}(\mathbb{R})$, it immediately follows that (see (3.34))

$$
\begin{aligned}
m(x, t, z) & =I+C\left(\mu w_{+}\right)(z) \\
& =I+C w_{+}(z)+C\left(\left(\left(1-C_{v}\right)^{-1} C^{-} w_{+}\right) w_{+}\right)(z) \\
& =I+\frac{1}{2 \pi i} \int_{\mathbb{R}}\left(\begin{array}{cc}
0 & \frac{r(s)}{s-z} e^{i \theta(s)} \\
\frac{r(s)}{s-z} e^{-i \theta(s)} & 0
\end{array}\right) \mathrm{d} s+O\left(\frac{q^{2}}{|\operatorname{Im} z|^{\frac{1}{2}}}\right),
\end{aligned}
$$

and

$$
u(x, t)=\frac{1}{2 \pi} \int_{\mathbb{R}}\left(\mu w_{+}\right)_{12}=\frac{1}{2 \pi} \int_{\mathbb{R}} r(s) e^{i \theta(s)} \mathrm{d} s+O\left(q^{2}\right) .
$$

Let $\psi^{+}=\left(\psi_{1}^{+}, \psi_{2}^{+}\right), \psi_{0}^{+}=\left(\left(\psi_{0}\right)_{1}^{+},\left(\psi_{0}\right)_{2}^{+}\right)$be the ZS-AKNS solutions for $u_{0}^{e}, \eta_{\mu_{0}}$, respectively. The following expansion is standard in the perturbation theory. 
Lemma 8.5. For $z \in \mathbb{R}, x \geq 0$,

$$
\psi^{+}(x, z)=\psi_{0}^{+}(x, z)-q \psi_{0}^{+}(x, z) \int_{x}^{\infty} L(y, z) \mathrm{d} y+O\left(q^{2}\right)
$$

uniformly where $L(x, z)=\left(\psi_{0}^{+}\right)^{-1} W \psi_{0}^{+}$and $W(x)=\left(\begin{array}{cc}0 & w(x) \\ -w(x) & 0\end{array}\right)$.

Proof. Let $\psi^{+}(x, z)=\psi_{0}^{+}(x, z) \phi(x, z)$. Then,

$$
\phi_{x}=\left(\psi_{0}^{+}\right)^{-1}\left(\psi_{x}^{+}-\left(\psi_{0}^{+}\right)_{x} \phi\right)=q L \phi .
$$

As $\phi=\left(\psi_{0}^{+}\right)^{-1} \psi^{+} \rightarrow I$ as $x \rightarrow+\infty, z \in \mathbb{R}$, it follows by standard iterations that

$$
\phi(x, z)=I-q \int_{x}^{\infty} L(y, z) \mathrm{d} y+O\left(q^{2}\right) .
$$

Proof of Theorem 2. It is enough to consider $x \geq 0$. We use $\approx$ to denote equality up to order $\frac{q}{\sqrt{t}}\left(t^{-\frac{1}{4}}+|q|^{\frac{1}{2}}+\tau|\log \tau|\right)$ if $t \leq \frac{1}{2}|q|^{-2}$, and order $q^{2}$ if $t \geq \frac{1}{C}|q|^{-2}$. Note that $q^{2} \leq \frac{c q}{\sqrt{t}} \tau|\log \tau|$ if $t \leq \frac{1}{2}|q|^{-2}$. By the previous lemma, setting $x=0$ and using (7.6), it follows that

$$
\left(\begin{array}{l}
A_{1}(z) \\
B_{1}(z)
\end{array}\right)=q\left(\begin{array}{ll}
A_{0}(z) & -\overline{B_{0}(z)} \\
B_{0}(z) & \overline{A_{0}(z)}
\end{array}\right)\left(\begin{array}{l}
f_{1}(z) \\
f_{2}(z)
\end{array}\right)+O\left(q^{2}\right), \quad z \in \mathbb{R}
$$

where

$$
\left(\begin{array}{l}
f_{1}(z) \\
f_{2}(z)
\end{array}\right)=\int_{0}^{\infty} w(s)\left(\begin{array}{c}
-2 i z \mu_{0} \operatorname{sech}\left(\mu_{0} s\right) /\left(z^{2}+\mu_{0}^{2}\right) \\
e^{i s z}\left(z^{2}+2 i z \mu_{0} \tanh \left(\mu_{0} s\right)-\mu_{0}^{2}\right) /\left(z+i \mu_{0}\right)^{2}
\end{array}\right) \mathrm{d} s
$$

and $A_{0}, B_{0}, A_{1}$ and $B_{1}$ are defined in (7.7). Let $g_{3}(z)$ be given in (7.10). By (8.1), we have $g_{3}\left(-z_{0}\right)=\overline{g_{3}\left(z_{0}\right)}$. Hence using (7.8) and (8.2), we obtain

$$
\begin{aligned}
g_{3}\left(z_{0}\right)+g_{3}\left(-z_{0}\right) & =2 \operatorname{Re}\left(A_{0}\left(-z_{0}\right) B_{1}\left(z_{0}\right)+A_{1}\left(-z_{0}\right) B_{0}\left(z_{0}\right)\right)+O\left(q^{2}\right) \\
& =-2 q K\left(z_{0}\right)+O\left(q^{2}\right)
\end{aligned}
$$

where $K(z)$ is given in (1.21).

If $a(z)$ has one zero $z_{1}=i \mu_{1}$, then $\beta=-q$ and hence it follows from (4.53) that

$$
b(z)=g_{3}(z)+g_{3}(-z)+\frac{2 i q}{z-i q} g_{3}(z),
$$

By Lemma 8.1 and Theorem [6, the norming constant $\gamma_{1}(t)=\gamma\left(t ; z_{1}\right)$ for $z=z_{1}$ is given by

$$
\gamma_{1}(t)=\gamma_{1} e^{-i \mu_{1}^{2} t / 2}=\sqrt{\frac{\mu_{1}+q}{\mu_{1}-q}} e^{-i \mu_{1}^{2} t / 2} .
$$


Set

$$
r_{f}(z) \equiv r(z) \frac{z+i \mu_{1}}{z-i \mu_{1}}, \text { and } c_{1}(t)=\frac{\gamma_{1}(t)}{a^{\prime}\left(z_{1}\right)} .
$$

where $a(z)=\frac{z-z_{1}}{z-\overline{z_{1}}} e^{-l(z)}$. By (3.24),

$$
r_{f}(z)=\frac{\overline{b(z)}}{\overline{a(z)}} \frac{z+i \mu_{1}}{z-i \mu_{1}}=\overline{b(z)} e^{\overline{l(z)}} .
$$

where $l(z)$ is defined in (3.25). Let $m_{f}(x, t, z), u_{f}(x, t)$ be the solution of the normalized RHP and the associated potential function in Lemma 8.4 with $r$ replaced by $r_{f}$. Set $h(z) \equiv e^{\overline{l(z)}}-1$. As $\|l\|_{H^{1,0}(\mathbb{R})} \leq c \| \log (1+$ $\left.|r|^{2}\right) \|_{H^{1,0}(\mathbb{R})}$, it follows that $\|h(z)\|_{H^{1,0}(\mathbb{R})}=O\left(|q|^{3 / 2}\right)$ and hence we see by Lemma 8.3, Lemma 8.4, (8.3) and (8.4) that

$$
m_{f}\left(x, t, z_{1}\right) \approx\left(\begin{array}{cc}
1 & p_{1} \\
p_{2} & 1
\end{array}\right), \quad u_{f}(x, t) \approx i\left(z_{0}-i \mu_{1}\right) p_{1}
$$

where

$$
p_{1}=-\frac{q}{\sqrt{t}} \frac{K\left(z_{0}\right) E_{0} e^{\frac{i t z_{0}^{2}}{2}}}{\pi i\left(z_{0}-i \mu_{1}\right)}, \quad p_{2}=-\frac{q}{\sqrt{t}} \frac{K\left(z_{0}\right) \overline{E_{0}} e^{-\frac{i t z_{0}^{2}}{2}}}{\pi i\left(z_{0}-i \mu_{1}\right)} .
$$

From Remark 4.30,

$$
l(i u)=\frac{1}{2 \pi i} \int_{\mathbb{R}} \frac{i u}{s^{2}+u^{2}} \log \left(1+|r(s)|^{2}\right) \mathrm{d} s=O\left(q^{2}\right),
$$

uniformly on $u>0$. Let $v_{1}, v_{1}^{x}$ be given in (1.22). It follows by (8.6) that $\frac{-c_{1}(t)}{z_{1}-\overline{z_{1}}}=v_{1}^{-1}+O\left(q^{2}\right)$. Set

$$
\mathfrak{b} \equiv m_{f}\left(z_{1}\right) e^{i x z_{1} \sigma}\left(\begin{array}{c}
1 \\
\frac{-c_{1}(t)}{z_{1}-\overline{z_{1}}}
\end{array}\right) .
$$

Assembling the above results, we see that

$$
v_{1} e^{-\frac{\mu_{1} x}{2}} \mathfrak{b} \approx\left(\begin{array}{c}
v_{1}^{x}+p_{1} \\
p_{2} v_{1}^{x}+1
\end{array}\right)
$$

Therefore, by the Darboux transformation,

$$
\begin{aligned}
u(x, t) & =u_{f}(x, t)+i\left(z_{1}-\overline{z_{1}}\right) \mathcal{F}(\mathfrak{b}) \\
& \approx i\left(z_{0}-i \mu_{1}\right) p_{1}-\frac{2 \mu_{1}\left(v_{1}^{x}+p_{1}\right)\left(\overline{p_{2}} \overline{v_{1}^{x}}+1\right)}{\left|v_{1}^{x}+p_{1}\right|^{2}+\left|p_{2} v_{1}^{x}+1\right|^{2}} .
\end{aligned}
$$

This relation holds, in particular, in the region $x \geq 1 / M$. If $t \geq \frac{1}{C}|q|^{-2}$, then $\frac{q}{\sqrt{t}}=O\left(q^{2}\right)$ and it follows that $p_{1}, p_{2}=O\left(q^{2}\right)$, which in turn implies that

$$
u(x, t) \approx \frac{-2 \mu_{1} v_{1}^{x}}{\left|v_{1}^{x}\right|^{2}+1} \approx e^{i \mu_{1}^{2} t / 2} v_{\mu_{1}}(x) .
$$


This proves (1.24) and the second part of (1.23).

Now assume $t \leq \frac{1}{2}|q|^{-2}$ and $0 \leq x \leq M$. Since $z_{0}=O\left(t^{-1}\right), K\left(z_{0}\right)=$ $\operatorname{Re} \int_{0}^{\infty} e^{-i s z_{0}} w(s)+O\left(t^{-1}\right)$. Set $K_{1}(z) \equiv \int_{-\infty}^{\infty} e^{-i s z} w(s) d s$ and $w_{0} \equiv$ $\int_{0}^{\infty} w(s) \mathrm{d} s=\frac{1}{2} K_{1}(0)$. As $w \in H^{0,1}(\mathbb{R}), K_{1} \in H^{1,0}(\mathbb{R})$ and so $\mid K_{1}\left(z_{0}\right)-$ $\left.K_{1}(0)|\leq| z_{0}\right|^{\frac{1}{2}}\left\|K_{1}^{\prime}\right\|_{L^{2}(\mathbb{R})}=O\left(t^{-\frac{1}{2}}\right)$. As $w$ is real and even, $K\left(z_{0}\right)=$ $\frac{1}{2} \operatorname{Re} K_{1}\left(z_{0}\right)+O\left(t^{-1}\right)=w_{0}+O\left(t^{-\frac{1}{2}}\right)$. Thus,

$$
p_{1}=-\frac{q w_{0}}{\mu_{1}} \sqrt{\frac{2}{\pi t}} e^{i\left[x^{2} /(2 t)-\pi / 4\right]}+O\left(q t^{-1}\right), \quad p_{2}=\overline{p_{1}}+O\left(q t^{-\frac{3}{2}}\right) .
$$

Combining the results, we obtain from (8.8) for $0 \leq x \leq M$,

$$
u(x, t) \approx e^{i \mu_{1}^{2} t / 2}\left[v_{\mu_{1}}(x)-q w_{0} \sqrt{\frac{2}{\pi t}}\left(e^{i \Omega} \operatorname{sech}^{2} \mu_{1} x-e^{-i \Omega} \tanh ^{2} \mu_{1} x\right)\right],
$$

where $\Omega(x, t)=-x^{2} /(2 t)+\mu_{1}^{2} t / 2+\pi / 4$. This completes the proof of (1.23).

If $a(z)$ has two zeros in $\mathbb{C}^{+}$, we have $\beta=q$ and hence it follows again from (4.53) that

$$
b(z)=g_{3}(z)+g_{3}(-z)-\frac{2 i q}{z+i q} g_{3}(-z) .
$$

Again by Lemma 8.1 and Theorem 6, the norming constants $\gamma_{j}(t)=$ $\gamma\left(t ; z_{j}\right)$ for $z=z_{j}, j=1,2$ are given by

$$
\gamma_{j}(t)=\gamma_{j} e^{-i \mu_{j}^{2} t / 2}=\sqrt{\frac{\mu_{j}-q}{\mu_{j}+q}} e^{-i \mu_{j}^{2} t / 2}, \quad j=1,2 .
$$

Set

$$
r_{f}(z)=\frac{z+i \mu_{1}}{z-i \mu_{1}} \frac{z+i \mu_{2}}{z-i \mu_{2}} \frac{\overline{b(z)}}{\overline{a(z)}}, \quad \text { and } c_{1}(t)=\frac{\gamma_{1}(t)}{a_{1}^{\prime}\left(z_{1}\right)}, \quad c_{2}(t)=\frac{\gamma_{2}(t)}{a^{\prime}\left(z_{2}\right)},
$$

where $a_{1}(z)=\frac{z-z_{1}}{z-z_{1}} e^{-l(z)}$ and $a(z)=\frac{z-z_{2}}{z-z_{2}} a_{1}(z)$. Denote by $m_{f}(x, t, z)$ the solution of the normalized $\operatorname{RHP}\left(\mathbb{R}, v_{f}\right)$ without poles associated with $r_{f}$, and by $m_{1}(x, t, z)$ the solution of the normalized $\operatorname{RHP}\left(\mathbb{R}, v_{1}=\right.$ $\left.\left(\begin{array}{c}1+\left|r_{1}\right|^{2} \\ r_{1} e^{-i \theta} e^{i \theta}\end{array}\right)\right)$ with $r_{1}(z)=\frac{z+i \mu_{2}}{z-i \mu_{2}} \frac{\overline{b(z)}}{\overline{a(z)}}$ and one pair of poles at $z_{1}=i \mu_{1}$ and $\overline{z_{1}}=-i \mu_{1}$ with the norming constants $\gamma_{1}(t),-\overline{\gamma_{1}(t)}$ (cf. (9.4)). Set

$$
\hat{v}_{1} \equiv-\sqrt{\frac{\mu_{1}+q}{\mu_{1}-q}} e^{i \mu_{1}^{2} t / 2}, \quad \hat{v}_{1}^{x} \equiv \hat{v}_{1} e^{-\mu_{1} x}
$$

and

$$
\mathfrak{b}_{1} \equiv m_{f}\left(z_{1}\right) e^{i z_{1} x \sigma}\left(\begin{array}{c}
1 \\
\frac{-c_{1}(t)}{z_{1}-\overline{z_{1}}}
\end{array}\right)
$$


Then, again by (8.6), we have

$$
\hat{v}_{1} e^{-\frac{\mu_{1} x}{2}} \mathfrak{b}_{1} \approx\left(\begin{array}{c}
\hat{v}_{1}^{x}+p_{1} \\
p_{2} \hat{v}_{1}^{x}+1
\end{array}\right)
$$

as above. Write $\mathfrak{b}_{1}=\left(\left(\mathfrak{b}_{1}\right)_{1},\left(\mathfrak{b}_{1}\right)_{2}\right)^{T}$. Define

$$
\begin{aligned}
& \hat{\mathfrak{b}}_{1} \equiv\left(\begin{array}{cc}
\left(\mathfrak{b}_{1}\right)_{1} & -\left(\overline{\mathfrak{b}_{1}}\right)_{2} \\
\left(\mathfrak{b}_{1}\right)_{2} & \left(\overline{\mathfrak{b}_{1}}\right)_{1}
\end{array}\right), \quad \lambda_{1} \equiv \frac{z_{2}-\overline{z_{1}}}{z_{2}-z_{1}} \\
& \hat{\mu} \equiv\left(\begin{array}{cc}
z_{2}-z_{1} & 0 \\
0 & z_{2}-\overline{z_{1}}
\end{array}\right)=\left(z_{2}-z_{1}\right)\left(\begin{array}{cc}
1 & 0 \\
0 & \lambda_{1}
\end{array}\right) .
\end{aligned}
$$

From Lemma 8.4 and the fact that $\mu_{2}=O(|q|)$ (Lemma 7.4), we obtain

$$
m_{f}\left(x, t, z_{2}\right)=\left(\begin{array}{cc}
1 & p_{3} \\
p_{4} & 1
\end{array}\right)+O\left(|q|^{\frac{3}{2}}\right)
$$

where

$$
p_{3} \equiv \frac{1}{2 \pi i} \int_{\mathbb{R}} \frac{r_{f}(s)}{s-i \mu_{2}} e^{i \theta(s)}, \quad p_{4} \equiv \frac{1}{2 \pi i} \int_{\mathbb{R}} \frac{\overline{r_{f}(s)}}{s-i \mu_{2}} e^{-i \theta(s)} .
$$

Define $\hat{v}_{2} \equiv-\sqrt{\frac{\mu_{2}+q}{\mu_{2}-q}} e^{i \mu_{2}^{2} t / 2}$. Then, $-\frac{c_{2}(t)}{z_{2}-\overline{z_{2}}}=\lambda_{1} \hat{v}_{2}^{-1}\left(1+O\left(q^{2}\right)\right)$ by (8.6) . Let $\psi_{1}(x, t, z)=m_{1}(x, t, z) e^{i x z \sigma}$. Note that $\hat{v}_{2}=O(q)$ by Lemma 7.4, $\lambda_{1}=-1+O(q)$ and $\hat{v}_{1}=-e^{i \mu_{2}^{2} t / 2}+O(q)$. Assembling the above results, we see that

$$
\begin{aligned}
\mathfrak{b}_{2} & \equiv m_{1}\left(z_{2}\right) e^{i x z_{2} \sigma}\left(\begin{array}{c}
1 \\
\frac{-c_{2}(t)}{z_{2}-\overline{z_{2}}}
\end{array}\right) \\
& =\hat{\mathfrak{b}}_{1} \hat{\mu} \hat{\mathfrak{b}}_{1}^{-1} m_{f}\left(z_{2}\right) e^{-x \mu_{2} \sigma} \hat{\mu}^{-1}\left(\begin{array}{c}
1 \\
\frac{-c_{2}(t)}{z_{2}-\overline{z_{2}}}
\end{array}\right) . \\
& =\hat{v}_{2}^{-1} e^{\frac{\mu_{2} x}{2}}\left[\left(\begin{array}{c}
p_{3}-\frac{s}{\hat{v}_{2}^{x}} s \\
1+O(q)]
\end{array}\right]\right.
\end{aligned}
$$

where $s=\frac{2\left(p_{3}-\hat{v}_{1}^{x}\right)}{\left|\hat{v}_{1}^{x}\right|^{2}+1}$. By Lemma 8.3, we see that

$$
q p_{3}=-\frac{1}{2 \pi} \frac{q}{\mu_{2}} \int_{\mathbb{R}} \frac{i \mu_{2}}{s-i \mu_{2}} \overline{b(s)}(1+h(s)) e^{i \theta(s)} \mathrm{d} s \approx 0 .
$$

Set $s_{0} \equiv \frac{-2 \hat{v}_{1}^{x}}{\left|\hat{v}_{1}^{x}\right|^{2}+1}$. Using (8.10) and (8.11), we have

$$
i\left(z_{2}-\overline{z_{2}}\right) \mathcal{F}\left(\mathfrak{b}_{2}\right) \approx \frac{2 q s_{0}\left(1+\hat{v}_{1}^{x} \overline{s_{0}}\right)}{\left|s_{0}\right|^{2}+\left|1+\hat{v}_{1}^{x} \overline{s_{0}}\right|^{2}}=\frac{4 q \hat{v}_{1}^{x}\left(\left|\hat{v}_{1}^{x}\right|^{2}-1\right)}{\left(\left|\hat{v}_{1}^{x}\right|^{2}+1\right)^{2}} .
$$


But,

$$
\begin{aligned}
& i\left(z_{1}-\overline{z_{1}}\right) \mathcal{F}(\mathfrak{b})-i\left(z_{1}-\overline{z_{1}}\right) \mathcal{F}\left(\mathfrak{b}_{1}\right) \\
& \quad=-\frac{2 \mu_{1}\left(v_{1}^{x}+p_{1}\right)\left(\overline{p_{2}} \overline{v_{1}^{x}}+1\right)}{\left|v_{1}^{x}+p_{1}\right|^{2}+\left|p_{2} v_{1}^{x}+1\right|^{2}}+\frac{2 \mu_{1}\left(\hat{v}_{1}^{x}+p_{1}\right)\left(\overline{p_{2}} \overline{\hat{v}_{1}^{x}}+1\right)}{\left|\hat{v}_{1}^{x}+p_{1}\right|^{2}+\left|p_{2} \hat{v}_{1}^{x}+1\right|^{2}} \\
& \quad \approx \frac{4 q \hat{v}_{1}^{x}\left(\left|\hat{v}_{1}^{x}\right|^{2}-1\right)}{\left(\left|\hat{v}_{1}^{x}\right|^{2}+1\right)^{2}} \approx i\left(z_{2}-\overline{z_{2}}\right) \mathcal{F}\left(\mathfrak{b}_{2}\right) .
\end{aligned}
$$

where $\mathfrak{b}$ is given in (8.7). Thus

$$
\begin{aligned}
u(x, t) & =u_{f}+i\left(z_{1}-\overline{z_{1}}\right) \mathcal{F}\left(\mathfrak{b}_{1}\right)+i\left(z_{2}-\overline{z_{2}}\right) \mathcal{F}\left(\mathfrak{b}_{2}\right) \\
& \approx u_{f}+i\left(z_{1}-\overline{z_{1}}\right) \mathcal{F}(\mathfrak{b}) .
\end{aligned}
$$

This completes the proof of Theorem 2 .

\section{Appendix - Adding in or Removing poles via a Darboux TRANSFORMATION}

The following calculations are standard in scattering/inverse scattering theory (see e.g. [RS02]). Let $u(x) \in H^{1,1}(\mathbb{R})$ be given and consider the associated ZS-AKNS operator $\partial_{x}-\left(i z \sigma+\left(\begin{array}{cc}0 & u(x) \\ -\overline{u(x)} & 0\end{array}\right)\right)$ and its reflection coefficient function $r(z) \in H^{1,1}(\mathbb{R})$. Suppose that for each $x \in \mathbb{R}$, $2 \times 2$ matrix $\psi(x, z)=m(x, z) e^{i x z \sigma}$ solves the corresponding RHP with a finite number of simple bound states at $z=z_{1}, \ldots, z_{n} \in \mathbb{C}^{+}$, and at $z=\overline{z_{1}}, \ldots, \overline{z_{n}} \in \mathbb{C}^{-}, n \geq 0$,

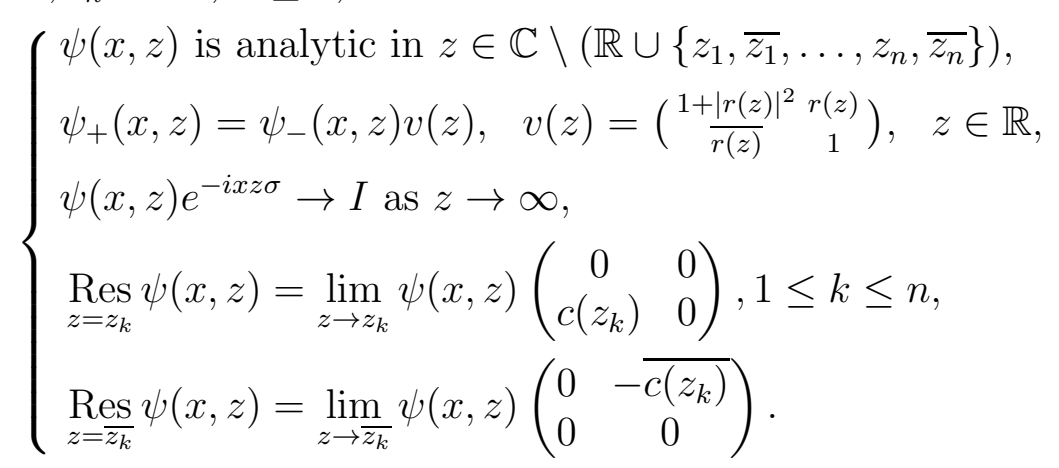

The goal is to add in another simple bound state at $z=\xi \in \mathbb{C}^{+} \backslash$ $\left\{z_{1}, \cdots, z_{n}\right\}$ and simultaneously at $z=\bar{\xi} \in \mathbb{C}^{-} \backslash\left\{\overline{z_{1}}, \cdots, \overline{z_{n}}\right\}$. We use a Darboux transformation $(z+P)\left(\partial_{x}-L\right)=\left(\partial_{x}-\tilde{L}\right)(z+P)$ as in (4.10). By (4.2), $P$ can be chosen in the form $P=\mathfrak{b}(x) P_{0} \mathfrak{b}^{-1}(x)$ where $P_{0}$ is a constant matrix and $\mathfrak{b}=\mathfrak{b}(x)$ solves the equation $b^{\prime}=Q b-i \sigma b P_{0}$. In contrast to the choice $P_{0}=P(0) \equiv-i q \sigma_{3}$ in (4.1), the appropriate choice here is $P_{0}=-\left(\begin{array}{cc}\xi & 0 \\ 0 & \bar{\xi}\end{array}\right) ; \mathfrak{b}$ is determined below. Set

$$
\tilde{\psi}(x, z) \equiv \mathfrak{b}(x) \mu(z) \mathfrak{b}^{-1}(x) \psi(x, z) \mu^{-1}(z),
$$


where $\mu(z)=z+P_{0}=\left(\begin{array}{cc}z-\xi & 0 \\ 0 & z-\bar{\xi}\end{array}\right)$. Note that $\tilde{\psi}(x, z) e^{-i x z \sigma} \rightarrow I$ as $z \rightarrow \infty$. Let $\tilde{c}(\xi)$ be any nonzero constant. We want to choose $\mathfrak{b}(x)$ so that $\tilde{\psi}$ has a simple pole in the first column at $z=\xi$ and a simple pole in the second column at $z=\bar{\xi}$ such that for $x \in \mathbb{R}$,

$$
\begin{aligned}
& \operatorname{Res}_{z=\xi} \tilde{\psi}(x, z)=\lim _{z \rightarrow \xi} \tilde{\psi}(x, z)\left(\begin{array}{cc}
0 & 0 \\
\tilde{c}(\xi) & 0
\end{array}\right), \\
& \operatorname{Res}_{z=\bar{\xi}} \tilde{\psi}(x, z)=\lim _{z \rightarrow \bar{\xi}} \tilde{\psi}(x, z)\left(\begin{array}{cc}
0 & -\overline{\tilde{c}(\xi)} \\
0 & 0
\end{array}\right) .
\end{aligned}
$$

Since

$$
\mathfrak{b}^{-1} \tilde{\psi}=\left(\begin{array}{cc}
\left(\mathfrak{b}^{-1} \psi\right)_{11} & \left(\mathfrak{b}^{-1} \psi\right)_{12} \frac{z-\xi}{z-\bar{\xi}} \\
\left(\mathfrak{b}^{-1} \psi\right)_{21} \frac{z-\bar{\xi}}{z-\xi} & \left(\mathfrak{b}^{-1} \psi\right)_{22}
\end{array}\right)
$$

we have

$$
\operatorname{Res}_{z=\xi} \mathfrak{b}^{-1}(x) \tilde{\psi}(x, z)=\left(\begin{array}{cc}
0 & 0 \\
\left(\mathfrak{b}^{-1} \psi\right)_{21}(x, \xi)(\xi-\bar{\xi}) & 0
\end{array}\right) .
$$

But,

$$
\lim _{z \rightarrow \xi} \mathfrak{b}^{-1}(x) \tilde{\psi}(x, z)\left(\begin{array}{cc}
0 & 0 \\
\tilde{c}(\xi) & 0
\end{array}\right)=\left(\begin{array}{cc}
0 & 0 \\
\tilde{c}(\xi)\left(\mathfrak{b}^{-1} \psi\right)_{22}(x, \xi) & 0
\end{array}\right) .
$$

and hence we must have

$$
(\xi-\bar{\xi})\left(e_{2}, \mathfrak{b}^{-1} \psi(x, \xi) e_{1}\right)=\tilde{c}(\xi)\left(e_{2}, \mathfrak{b}^{-1} \psi(x, \xi) e_{2}\right) .
$$

Therefore, it follows necessarily that

$$
\mathfrak{b}(x) e_{1}=c_{1}(x)\left(\psi(x, \xi) e_{1}-\frac{\tilde{c}(\xi)}{\xi-\bar{\xi}} \psi(x, \xi) e_{2}\right)
$$

for some nonzero function $c_{1}(x)$. Similarly for $z=\bar{\xi}$, we see that

$$
\mathfrak{b}(x) e_{2}=c_{2}(x)\left(-\frac{\overline{\tilde{c}(\xi)}}{\xi-\bar{\xi}} \psi(x, \bar{\xi}) e_{1}+\psi(x, \bar{\xi}) e_{2}\right)
$$

for some nonzero function $c_{2}(x)$. Observe that $c_{1}(x), c_{2}(x)$ factor out in the formula (9.2) for $\tilde{\psi}(x, z)$. Set

$$
\mathfrak{b}(x)=\left(\psi(x, \xi)\left(\begin{array}{c}
1 \\
\frac{-\tilde{c}(\xi)}{\xi-\bar{\xi}}
\end{array}\right) \psi(x, \bar{\xi})\left(\begin{array}{c}
\frac{-\overline{\tilde{c}(\xi)}}{\xi-\bar{\xi}} \\
1
\end{array}\right)\right) .
$$

From the symmetry in Proposition $3.1(\mathrm{i})$ we see that $\mathfrak{b}_{2}=\left(\begin{array}{cc}0 & -1 \\ 1 & 0\end{array}\right) \overline{\mathfrak{b}_{1}}$ where $\mathfrak{b}=\left(\mathfrak{b}_{1}, \mathfrak{b}_{2}\right)$. Thus, $\operatorname{det} \mathfrak{b}(x)=\left|\left(\mathfrak{b}_{1}\right)_{1}(x)\right|^{2}+\left|\left(\mathfrak{b}_{1}\right)_{2}(x)\right|^{2}>0$ and 
hence $\mathfrak{b}(x)$ is invertible for all $x \in \mathbb{R}$. The jump matrix $\tilde{v}$ for $\tilde{\psi}(x, z)$ is given by

$$
\begin{aligned}
\tilde{v}(z) & =\tilde{\psi}_{-}^{-1}(x, z) \tilde{\psi}_{+}(x, z)=\mu(z) v(z) \mu^{-1}(z) \\
& =\left(\begin{array}{cc}
1+\frac{|\tilde{r}(z)|^{2}}{\tilde{r}(z)} & \tilde{r}(z) \\
\tilde{r}(z) & 1
\end{array}\right), \quad z \in \mathbb{R}
\end{aligned}
$$

where

$$
\tilde{r}(z)=r(z) \frac{z-\xi}{z-\bar{\xi}} .
$$

A straightforward calculation shows that for $1 \leq k \leq n$,

$$
\begin{aligned}
& \operatorname{Res}_{z=z_{k}} \tilde{\psi}(x, z)=\lim _{z \rightarrow z_{k}} \tilde{\psi}(x, z)\left(\begin{array}{cc}
0 & 0 \\
\tilde{c}\left(z_{k}\right) & 0
\end{array}\right), \\
& \operatorname{Res}_{z=\overline{z_{k}}} \tilde{\psi}(x, z)=\lim _{z \rightarrow \overline{z_{k}}} \tilde{\psi}(x, z)\left(\begin{array}{cc}
0 & -\overline{\tilde{c}\left(z_{k}\right)} \\
0 & 0
\end{array}\right) .
\end{aligned}
$$

where

$$
\tilde{c}\left(z_{k}\right)=c\left(z_{k}\right) \frac{z_{k}-\bar{\xi}}{z_{k}-\xi} .
$$

The above calculations show that $\tilde{m}(x, z)=\tilde{\psi}(x, z) e^{-i x z \sigma}$ solves the RHP of type (3.31) with $r(z), Z_{+}, K_{+}$replaced by $\tilde{r}(z), \tilde{Z}_{+}=\left\{z_{1}, \cdots, z_{n}, \xi\right\}$, $\tilde{K}_{+}=\left\{\tilde{c}\left(z_{1}\right), \cdots \tilde{c}\left(z_{n}\right), \tilde{c}(\xi)\right\}$, respectively. Note that (see (3.24)

$$
\tilde{a}(z)=\frac{z-\xi}{z-\bar{\xi}} a(z)
$$

where $a(z), \tilde{a}(z)$ are the scattering functions for $\psi(x, z), \tilde{\psi}(x, z)$, respectively. Hence we see from (3.27) that

$$
\tilde{\gamma}\left(z_{k}\right)=\tilde{c}\left(z_{k}\right) \tilde{a}^{\prime}\left(z_{k}\right)=c\left(z_{k}\right) a^{\prime}\left(z_{k}\right)=\gamma\left(z_{k}\right), \quad k=1, \cdots, n
$$

where $\gamma\left(z_{k}\right), \tilde{\gamma}\left(z_{k}\right)$ are the corresponding norming constants. By Remark $3.9, \tilde{m}$ is unique. Finally, we compute the corresponding potential $\tilde{u}(x)$. From the fact that $m_{x}=i z[\sigma, m]+Q m, Q=\left(\begin{array}{cc}0 & u \\ -\bar{u} & 0\end{array}\right)$, we have

$$
Q=-i\left[\sigma, m_{1}\right], \quad m=I+\frac{m_{1}}{z}+o\left(z^{-1}\right),
$$

as $z \rightarrow \infty$ in any cone $|\operatorname{Im} z|>c|\operatorname{Re} z|, c>0$. Let $\mu_{1}=\left(\begin{array}{cc}\xi & 0 \\ 0 & \bar{\xi}\end{array}\right)$. For $\tilde{m}=\tilde{\psi} e^{-i x z \sigma}$

$$
\begin{aligned}
\tilde{m} & \left.=\mathfrak{b}\left(I-\frac{\mu_{1}}{z}\right) \mathfrak{b}^{-1}\left(I+\frac{m_{1}}{z}+o\left(z^{-1}\right)\right)\right)\left(I-\frac{\mu_{1}}{z}\right)^{-1} \\
& =I+\frac{m_{1}-\mathfrak{b} \mu_{1} \mathfrak{b}^{-1}+\mu_{1}}{z}+o\left(z^{-1}\right),
\end{aligned}
$$


and hence

$$
\begin{aligned}
\tilde{u}(x) & =-i\left[\sigma, m_{1}-\mathfrak{b} \mu_{1} \mathfrak{b}^{-1}+\mu_{1}\right]_{12} \\
& =u(x)+i(\xi-\bar{\xi}) \frac{\left(\mathfrak{b}_{1}\right)_{1} \overline{\left(\mathfrak{b}_{1}\right)_{2}}}{\left|\left(\mathfrak{b}_{1}\right)_{1}\right|^{2}+\left|\left(\mathfrak{b}_{1}\right)_{2}\right|^{2}} .
\end{aligned}
$$

One can also use Darboux transformations similar to (9.2) to remove eigenvalues. We do not provide any further details, except to note that at each step, if the poles at $z=z_{k}, \overline{z_{k}}$ are removed, then $r(z) \rightarrow \tilde{r}(z)=$ $r(z) \frac{z-\overline{z_{k}}}{z-z_{k}}$, etc.

\section{ACKNOWLEDGEMENTS}

The work of the first author was supported in part by NSF Grant DMS-0500923.

\section{REFERENCES}

[AKNS74] M. J. Ablowitz, D. J. Kaup, A. C. Newell, and H. Segur. The inverse scattering transform-fourier analysis for nonlinear problems. Studies in Appl. Math., 53(4):249-315, 1974.

[AS65] M. Abramowitz and I. A. Stegun. Handbook of Mathematical Functions. Dover Publications, New York, 1965.

[BC84] R. Beals and R. R. Coifman. Scattering and inverse scattering for first order systems. Comm. Pure Appl. Math., 37(1):39-90, 1984.

[BDT88] R. Beals, P. A. Deift, and C. Tomei. Direct and Inverse Scattering on the Line. Number 28 in Mathematical Surveys and Monographs. American Mathematical Society, Providence, RI, 1988.

[BK97] A. Böttcher and Y. I. Karlovich. Carleson curves, Muckenhoupt weights, and Toeplitz operators, volume 154 of Progress in mathematics. Birkhäuser, Boston, 1997.

[BT91] R. F. Bikbaev and V. O. Tarasov. Initial-boundary value problem for the nonlinear Schrödinger equation. J. Phys. A: Math. Gen., 24(11):25072516, 1991.

[CG81] K. Clancey and I. Gohberg. Factorization of matrix functions and singular integral operators. Birkhäuser Verlag, Boston, 1981.

[CL55] E. A. Coddington and N. Levinson. Theory of Ordinary Differential Equations. McGraw-Hill, New York, 1955.

[Dei78] P. A. Deift. Applications of a commutation formula. DukeMath. J., 45(2):267-310, 1978.

[DIZ93] P. A. Deift, A. Its, and X. Zhou. Long-time asymptotics for integrable nonlinear wave equations. Important Developments in Soliton Theory 1980-1990, pages 181-204, 1993. A.S. Fokas and V.E. Zakharov, eds.

[DZ91] P. A. Deift and X. Zhou. Direct and inverse scattering on the line with arbitrary singularities. Comm. Pure Appl. Math., 44(5):485-533, 1991.

[DZ93] P. A. Deift and X. Zhou. A steepest descent method for oscillatory Riemann-Hilbert problems. Asymptotics for the MKdV equation. Ann. of Math., 137(2):295-368, 1993. 
[DZ03] P. A. Deift and X. Zhou. Long-time asymptotics for solutions of the NLS equation with initial data in weighted Sobolev spaces. Comm. Pure Appl. Math., 56:1029-1077, 2003.

[FIS05] A. S. Fokas, A. R. Its, and L. Y. Sung. The nonlinear Schrödinger equation on the half-line. Nonlinearity, 18(4):1771-1822, 2005.

[Fok89] A. S. Fokas. An initial-boundary value problem for the nonlinear Schrödinger equation. Phys. D, 35:167-185, 1989.

[Fok02] A. S. Fokas. Integrable nonlinear evolution equations on the half-line. Comm. Math. Phys., 230:1-39, 2002.

[FT87] L.D. Faddeev and L.A. Takhtajan. Hamiltonian methods in the theory of solitons. Springer-Verlag, Berlin ; New York, 1987.

[HZ09] J. Holmer and M. Zworski. Breathing patterns in nonlinear relaxation. Nonlinearity, 22(6):1259-1301, 2009.

[Isa93] V. Isakov. Carleman type estimates in an anisotropic case and applications. J. Diff. Eq., 105:217-238, 1993.

[Kha91] I. T. Khabibullin. Bäcklund transformation and integrable initialboundary value problems. Mat. Zametki, 49(4):130-137, 1991.

[LS87] G. S. Litvinchuk and I. M. Spitkovsky. Factorization of measurable matrix functions. Number 5. Akademie-Verlag, Berlin, 1987.

[PS03] L. P. Pitaevskii and S. Stringari. BoseEinstein Condensation. Clarendon Press, Oxford, 2003.

[RS02] C. Rogers and W. K. Schief. Bäcklund and Darboux transformations. Geometry and modern applications in soliton theory. Cambridge Texts in Applied Mathematics. Cambridge University Press, Cambridge, 2002.

[Sha75] A. B. Shabat. The inverse scattering problem for a system of differential equations (Russian). Funkcional. Anal. i Prilozzen., 9(3):75-78, 1975.

[Skl87] E. K. Sklyanin. Boundary conditions for integrable equations. Functional Anal. Appl., 21(2):164-166, 1987.

[Tar88] V. O. Tarasov. A boundary value problem for the nonlinear Schrödinger equation. Zap. Nauchn. Sem.(LOMI), 169(3):151-165, 1988. translation in J. Soviet Math. 54 (1991), no. 3, 958-967.

[Tar91] V. O. Tarasov. The integrable initial-boundary value problem on a semiline: nonlinear schrödinger and sine-Gordon equations. Inverse Problem, 7(3):435-449, 1991.

[Zho89] X. Zhou. The Riemann-Hilbert problem and inverse scattering. SIAM J. Math. Anal., 20(4):966-986, 1989.

[Zho98] X. Zhou. $L^{2}$-Sobolev space bijectivity of the scattering and inverse scattering transforms. Comm. Pure Appl. Math., 51(7):697-731, 1998.

[ZS72] V. E. Zakharov and A. B. Shabat. Exact theory of two-dimensional self-focusing and one-dimensional self-modulation of waves in nonlinear media. Soviet Physics JETP, 34(1):62-69, 1972. translated from $\breve{Z}$. Éksper. Teoret. Fiz. 61 (1971), no. 1, 118-134.

Courant Institute of Mathematical Sciences, New York University, NEW YORK, NY, USA. 